\title{
New pathophysiological concepts and potential therapeutic targets for oxidative phosphorylation disorders
}

Citation for published version (APA):

Voets, A. M. (2012). New pathophysiological concepts and potential therapeutic targets for oxidative phosphorylation disorders. [Doctoral Thesis, Maastricht University]. Maastricht University. https://doi.org/10.26481/dis.20120524av

Document status and date:

Published: 01/01/2012

DOI:

10.26481/dis.20120524av

Document Version:

Publisher's PDF, also known as Version of record

Please check the document version of this publication:

- A submitted manuscript is the version of the article upon submission and before peer-review. There can be important differences between the submitted version and the official published version of record.

People interested in the research are advised to contact the author for the final version of the publication, or visit the DOI to the publisher's website.

- The final author version and the galley proof are versions of the publication after peer review.

- The final published version features the final layout of the paper including the volume, issue and page numbers.

Link to publication

\footnotetext{
General rights rights.

- You may freely distribute the URL identifying the publication in the public portal. please follow below link for the End User Agreement:

www.umlib.nl/taverne-license

Take down policy

If you believe that this document breaches copyright please contact us at:

repository@maastrichtuniversity.nl

providing details and we will investigate your claim.
}

Copyright and moral rights for the publications made accessible in the public portal are retained by the authors and/or other copyright owners and it is a condition of accessing publications that users recognise and abide by the legal requirements associated with these

- Users may download and print one copy of any publication from the public portal for the purpose of private study or research.

- You may not further distribute the material or use it for any profit-making activity or commercial gain

If the publication is distributed under the terms of Article $25 \mathrm{fa}$ of the Dutch Copyright Act, indicated by the "Taverne" license above, 
New pathophysiological concepts and potential therapeutic targets for oxidative phosphorylation disorders 
ISBN 978-90-8891-411-9

Cover design: Tony Houbrechts

Lay-out: An Voets

Printed by: Proefschriftmaken.nl

(c) Copyright An M. Voets, 2012

The studies described in this thesis were supported by IOP Genomics grant IGE05003, the Interreg IV program (project EMR.INT4) and the Kerry foundation and were performed within the CARIM School for Cardiovascular Diseases and the Faculty of Health, Medicine and Life Sciences of Maastricht University. 


\title{
New pathophysiological concepts and potential therapeutic targets for oxidative phosphorylation disorders
}

\author{
Proefschrift \\ ter verkrijging van de graad van doctor \\ aan de Universiteit Maastricht \\ op gezag van de Rector Magnificus, \\ Prof. mr. G.P.M.F. Mols \\ volgens het besluit van het College van Decanen \\ in het openbaar te verdedigen \\ op 24 mei 2012 om 10.00u \\ door \\ An Mieke Voets \\ Geboren op 8 juni 1984 te Bilzen
}


Promotor

Prof. Dr. H.J.M. Smeets

Copromotor

Dr. I.F.M. de Coo

Beoordelingscommissie

Prof. dr. G. J. van der Vusse (UM) (voorzitter)

Prof. dr. H. H. W. Schmidt (UM)

Prof. dr. F. J. van Schooten (UM)

Prof. dr. B. Wieringa (Radboud UMC Nijmegen) 
It won't be soon before long

Maroon 5 

Table of contents

Chapter 1

General Introduction

Chapter 2

Large scale mtDNA sequencing reveals sequence and functional conservation as major determinants of homoplasmic mtDNA variant distribution.

Chapter 3

POLG1 defects lead to mitochondrial muscle abnormalities, oxidative stress in fibroblasts and apoptosis in brain and liver.

\section{Chapter 4}

Transcriptional changes in OXPHOS complex I deficiency are related to antioxidant pathways and could explain the disturbed calcium homeostasis.

Chapter 5

Patient-derived fibroblasts indicate oxidative stress status and may justify antioxidant therapy in OXPHOS disorders.

Chapter 6

General Discussion

Summary

Samenvatting

Dankwoord

Curriculum Vitae and list of publications 



\section{Chapter 1}

General Introduction 
으 


\section{Oxidative phosphorylation disorders}

Mitochondrial disorders are characterized by a broad spectrum of multisystem clinical features, sometimes clustered into a syndrome but more often not. They affect at least 1 in 5000 individuals and are associated with a deficiency of the oxidative phosphorylation (OXPHOS) system [1]. Therefore, they will be further referred to as OXPHOS disorders. Mitochondrial OXPHOS is essential for aerobic energy production in virtually all cells. During this process, oxidation of electron carriers nicotinamide adenine dinucleotide (NADH) and flavin adenine dinucleotide $\left(F A D H_{2}\right)$, reduced during metabolism of carbohydrates, fatty acids and proteins, drives the production of ATP. The OXPHOS system is composed of five complexes of which the subunits are encoded by both the mitochondrial and nuclear DNA (except for complex II, which is entirely encoded by nuclear DNA). Accordingly, OXPHOS disorders can be caused by mutations in both genomes. Because mitochondria are the main providers of energy, tissues with high energy demands are affected most often in OXPHOS disorders. These organs include the brain, heart, liver and muscle [2]. The consequences of pathogenic mutations can be tissue specific as different metabolic thresholds, depending upon the energy demand, apply for the different tissues [3]. However, this is not the sole factor involved and the mechanism behind the tissue specificity is not

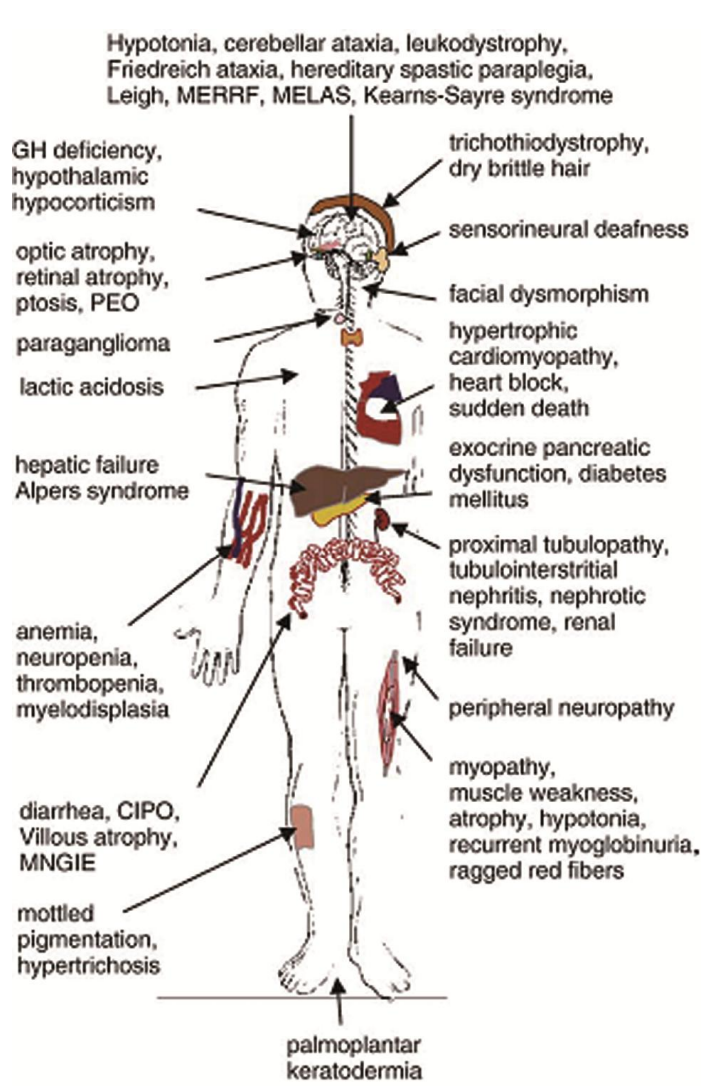
entirely clear. Thus, OXPHOS disease is usually multisystemic and the variety of clinical features includes, among others, encephalopathy, stroke, epilepsy, optic atrophy, deafness, neuropathy, myopathy, cardio-myopathy, exercise intolerance, diabetes and liver failure (figure 1.1) [4]. A few specific syndromes could be defined (table 1.1) but most often OXPHOS disease is not so well delineated [4]. Furthermore, there is most often no cure and the treatment options for even delaying OXPHOS disorders are limited.

Figure 1.1. Potential clinical features associated with OXPHOS disease (adapted from [5]). 


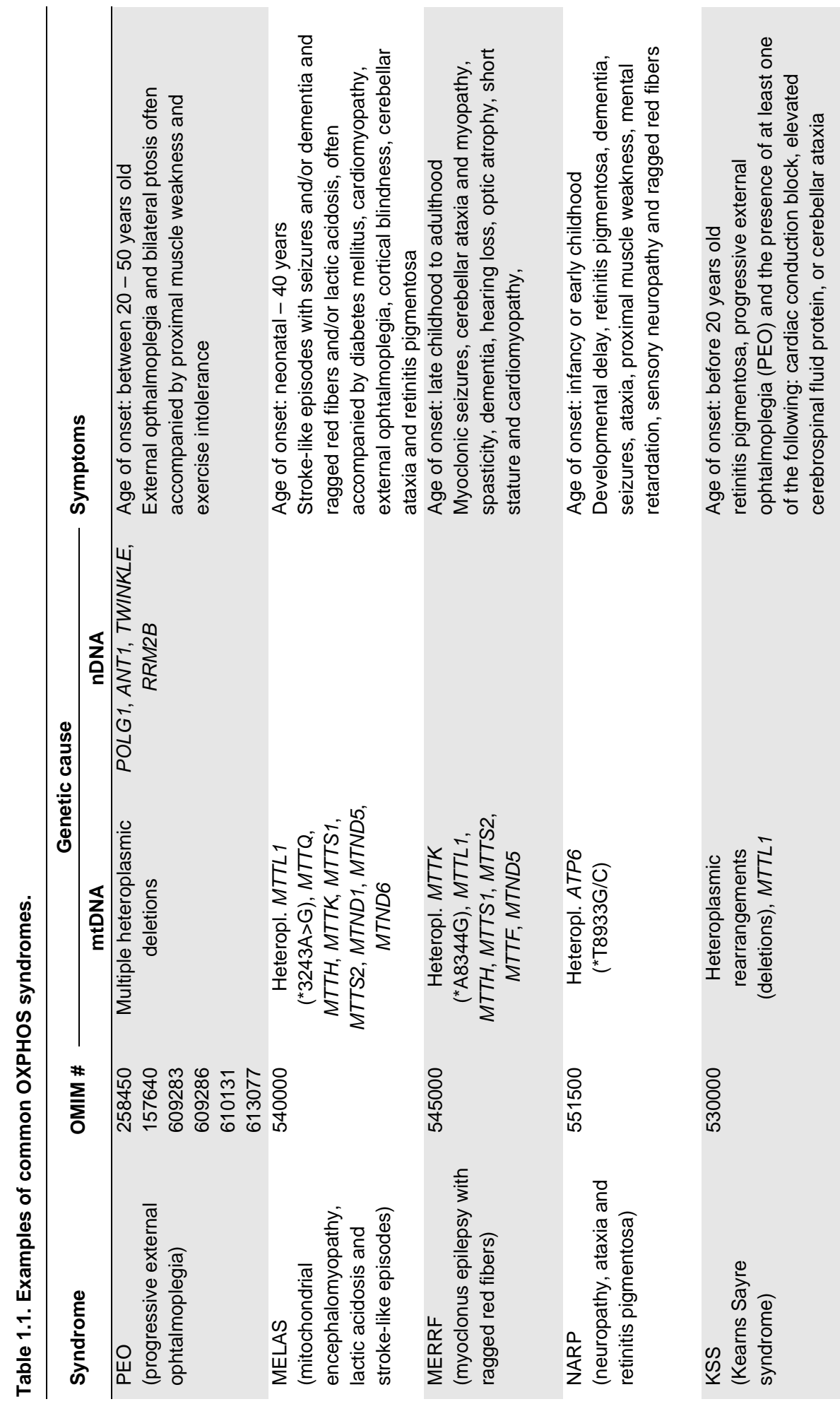




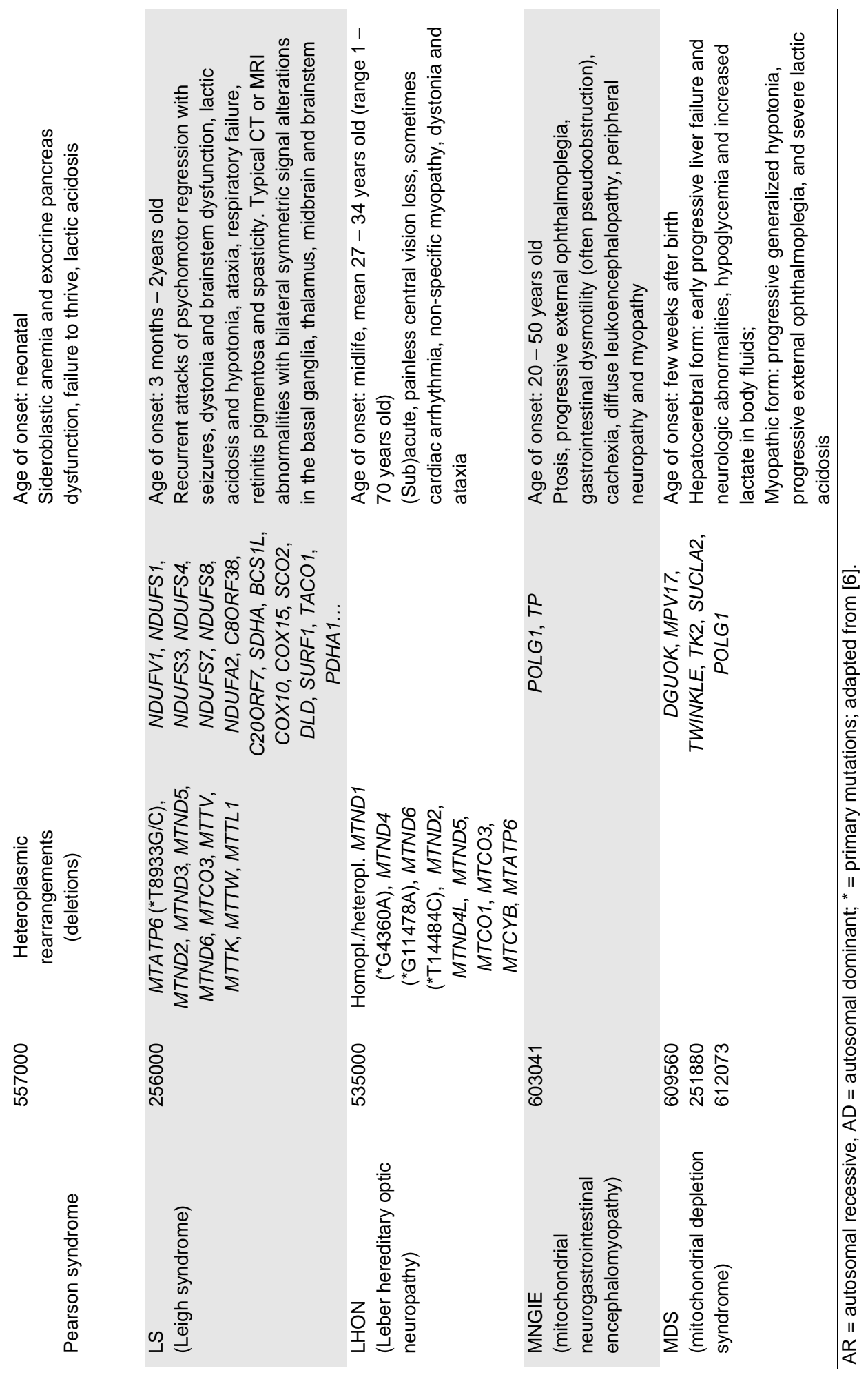




\section{Mitochondrial DNA}

The mitochondrial genome is a double-stranded, circular molecule consisting of 16.569 base pairs and coding for 13 subunits of the OXPHOS complexes, two rRNAs and 22 tRNAs, required for mitochondrial translation (figure 1.2). The mitochondrial DNA (mtDNA) has a number of unique characteristics that discriminate it from nuclear DNA (nDNA).

- The mtDNA is polyploid. A mitochondrion contains five to ten copies of mtDNA and a cell can contain hundreds to thousands of mitochondria. All mtDNA molecules can be identical (homoplasmy) or mixtures of wild and mutant type base pairs can be present in a cell (heteroplasmy).

- mtDNA encoded genes do not have introns and several of them are overlapping. Less than ten percent of the genome is non-coding and is predominantly located in the displacement (D)-loop, involved in the replication and transcription of the mtDNA. Transcription of mtDNA can occur on both (heavy and light) strands, starting from the mitochondrial promotors, and produces polycistronic precursor RNA. This RNA is subsequently processed into individual tRNA, rRNA and mRNA molecules.

- mtDNA is maternally inherited.

- Replication of mtDNA is called relaxed as it is not connected to the cell cycle.

- mtDNA molecules lack histones and other protective proteins. In combination with its location nearby the reactive oxygen species (ROS)-producing OXPHOS system and less efficient DNA repair mechanism, this leads to higher mutation frequencies in mtDNA compared with nDNA.

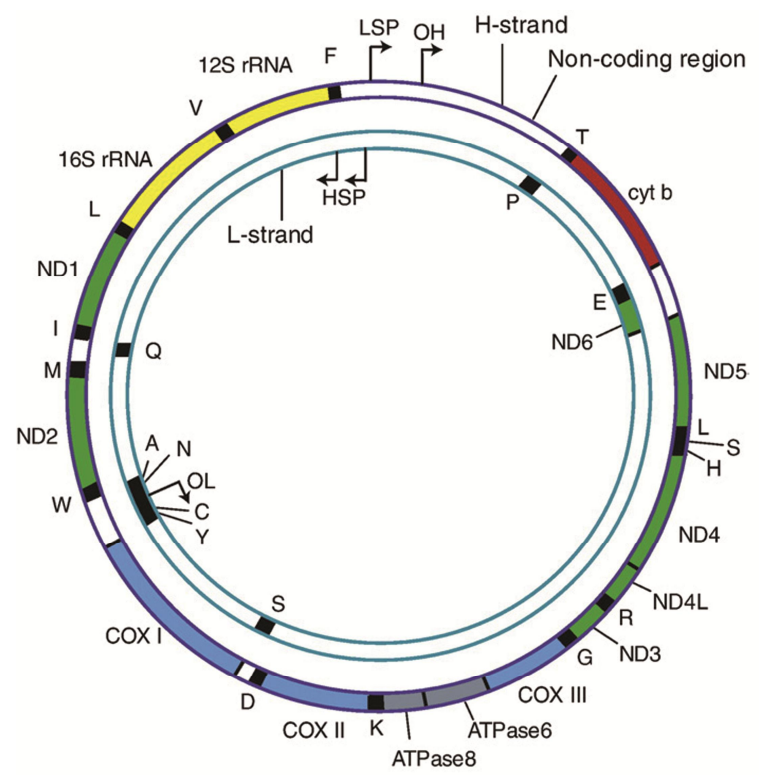

Figure 1.2. The mitochrondrial genome. Complex I (ND) genes are shown in green, the complex III Cyt $b$ in red, complex IV (COX) genes in light blue, complex $\mathrm{V}$ (ATPase) genes in grey, rRNA genes in yellow and tRNA genes in black. Adapted from [7]. 
Clinically relevant mtDNA variations fall into five categories: 1) de novo or maternally inherited deleterious point mutations (mainly substitutions); 2) ancient adaptive polymorphisms that predispose individuals to disease in different environments; 3 ) single or multiple mtDNA deletions affecting multiple mtDNA encoded genes; 4) duplication of part of the mtDNA molecule [8] and 5) differences in mtDNA copy number, which when extremely decreased is called mtDNA depletion. These variations can occur separately or in combination (e.g. m.3243A $>\mathrm{G}$ and reduced mtDNA copy number [9]).

As a result of the exclusive maternal inheritance of mtDNA, OXPHOS disease due to mtDNA mutations is transmitted through the maternal lineage. The majority of pathogenic mtDNA variations are heteroplasmic, although a growing list of homoplasmic pathogenic mutations, including the more frequent occurring LHON mutations, exists. However, the variable penetrance of homoplasmic pathogenic mutations implies the presence of modifiers. Modifiers can be of mtDNA or nDNA origin and probably involve one or more polymorphisms that do not necessarily induce pathology on themselves [10]. For heteroplasmic mutations, a certain heteroplasmy threshold level is necessary before respiratory defects or tissue dysfunction become apparent, depending on the aerobic energy need of a tissue. Often, a higher proportion of mutant mtDNA will lead to a more severe biochemical defect and/or clinical phenotype [11], but an exact correlation is lacking [12]. When new mtDNA variants are identified in patients, a number of criteria (box 1) are checked to determine their pathogenicity. However, there are many examples of pathogenic mtDNA variants in literature that do not fit all of the current pathogenicity criteria, which particularly challenges the genetic counseling of families [13, 14].

\section{Box 1. Pathogenicity criteria for mtDNA point mutations [14]}

- Mutation must be present in patients and absent in controls

- Mutation must be found in different mitochondrial genetic backgrounds

- Mutation must be the best mtDNA candidate variant to be pathologic

- The percentage of the mutation must correlate with the phenotype

- Mutation must affect highly evolutionary conserved nucleotides

- Mutation must affect functionally important domains

- The transfer of the mutated mtDNA to another cell line must be accompanied by the transfer of the cell or molecular defect

\section{Nuclear genes involved in oxidative phosphorylation}

In contrast to the extensively studied and characterized mitochondrial genome, the list of nuclear encoded OXPHOS-related genes is less complete. Nuclear genes involved in OXPHOS function code for structural components of the OXPHOS complexes (e.g. NDUFV1, NDUFS4, SDHA), assembly factors of OXPHOS complexes (e.g. SURF1, C20orf7, BCS1L), factors involved in mtDNA replication and maintenance (POLG1, 
TWINKLE, mtSSB) and proteins involved in the mitochondrial nucleotide balance (ANT1, DGUOK, TK2, TP, SUCLA2), mtDNA transcription (e.g. POLRMT, TFAM) and translation (e.g. PUS1, EFG1, MRPS16). Furthermore, there are also several nuclear genes that are indirectly related to respiration and energy production by mitochondria. This group includes genes coding for mitochondrial metalloproteases, mitochondrial import/export proteins, mitochondrial iron-storage proteins and proteins associated with mitochondrial fusion and fission. Mutations in these genes are associated, among others, with spastic paraplegia (SPG7), Friedreich's ataxia (FRDA), X-linked sideroblastic anemia and ataxia $(A B C 17)$ and optic atrophy (OPA1) [15].

\section{Diagnostic strategy in suspected OXPHOS disease}

Because of the variety in clinical manifestations of OXPHOS disease, diagnosis often requires a multidisciplinary approach. No single symptom is specific for OXPHOS disease or is able to distinguish it from other disorders, but when combinations of common chronic and unexplained symptoms (such as migraine, diabetes, deafness) occur, the possibility of OXPHOS disease should be considered $[16,17]$. When clinical suspicion of OXPHOS disease arises, a number of investigations, including histochemical, biochemical and genetic analysis, can lead to the eventual diagnosis (figure 1.3).

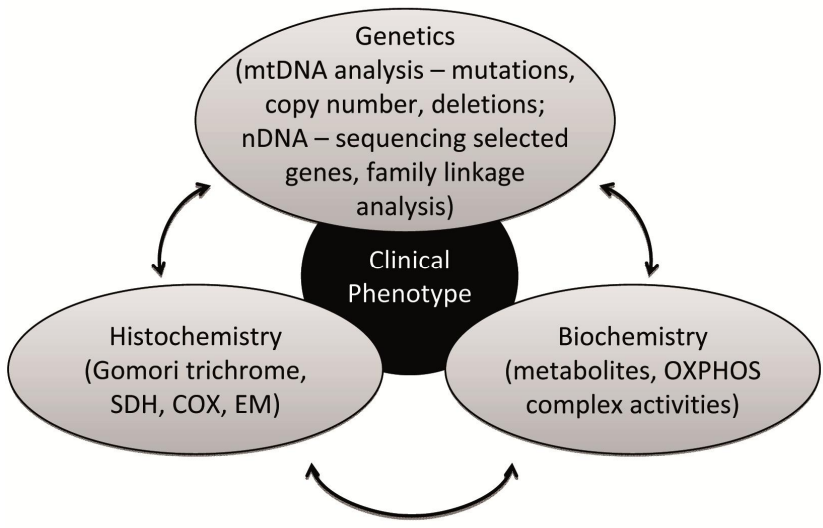

Figure 1.3. Diagnosis of OXPHOS disease needs a multidisciplinary approach. SDH = succinate dehydrogenase, $\mathrm{COX}=$ cytochrome $\mathrm{C}$ oxidase, $\mathrm{EM}=$ electron microscopy

Histochemical investigations

Histochemical assays are used to detect abnormal focal accumulation of mitochondria or low activity of respiratory chain enzymes in skeletal muscle biopsies. The red Gomori trichrome staining visualizes accumulation of focal subsarcolemmal mitochondria such as the so-called ragged red fibers (RRFs) which presumably denote a decrease of 
energy production [18]. However, RRFs have also been described in healthy individuals and are only seldom observed in young children with mitochondrial disease, making interpretation by an expert necessary $[17,18]$. Combined histoenzymatic reactions for succinate dehydrogenase (SDH) and cytochrome c oxidase (COX) typically result in a mosaic appearance of abnormalities but may be helpful in visualizing COX-negative fibers with mitochondrial proliferation (increased SDH staining). Histochemical investigations can provide additional evidence for a diagnosis of OXPHOS disease.

\section{Biochemical assays}

An initial biochemical examination in patients suspected of OXPHOS disease is a metabolic screening including the measurement of the metabolites lactate, pyruvate and alanine in blood, urine or cerebrospinal fluid and the measurement of organic acids in urine [16]. OXPHOS dysfunction leading to a disturbed oxidation ratio of NADH to $\mathrm{NAD}^{+}$results in feedback inhibition of pyruvate metabolism in the mitochondria. The excess pyruvate is transported back into the cytosol. Here it is metabolized into lactate or converted into alanine, which leads to an increase of these metabolites in body fluids [16]. The increase in the $\mathrm{NADH} / \mathrm{NAD}^{+}$ratio or the reduced oxidation rate of $\mathrm{FADH}_{2}$ may also lead to a metabolic arrest of the Krebs cycle leading to an increase and subsequent excretion of intermediates of the citric acid cycle in urine. Furthermore elevated levels of ethylmalonate and 3-methylgluconate are frequently found in mitochondrial patients [19]. However, due to the limited specificity of the initial screen, definite biochemical investigations are performed, preferably in a skeletal muscle biopsy or in fibroblasts when no muscle sample is available. Biochemical investigations in muscle may include the measurement of overall oxygen consumption, radio-labeled substrate oxidation or ATP synthesis by the OXPHOS system in fresh muscle biopsies and measurement of the activities of individual respiratory chain enzymes in frozen muscle homogenates. Assessing overall oxygen consumption, substrate oxidation and ATP synthesis in mitochondria require an intact mitochondrial membrane and is thus only applicable in fresh muscle biopsies [16]. The spectrophotometric assays of the activities of the individual complexes in muscle/fibroblast homogenates are based on the specific kinetic measurement of the velocity of the oxidation or reduction of the physiological substrates by the use of specific inhibitors of the individual complexes [18]. Interpretation of the individual complex measurements is rather difficult because there is only a narrow margin between control and patient ranges. Due to a large variation of absolute activities in samples which may be due to a large variation in the amount of mitochondria, the activity of the different complexes is expressed relative to the activity of another respiratory chain complex (usually complex II or IV) or citrate synthase. The severity of the enzyme deficiency is not necessarily linked to the severity of the clinical phenotype, therefore it is also important to detect even mild deficiencies. Furthermore, it is important that the different complexes or electron carriers are in a strict equilibrium for a sustained continuous and efficient substrate oxidation. Any imbalance can result in increased superoxide production or a decrease in respiration [18]. Available methods to measure superoxide production and its consequences are mainly based on HPLC analysis [20], probe (e.g. hydroethidine) oxidation [21, 22], mass spectrometry [23] and immunology [21]. 


\section{Genetic analysis}

In general, according to the clinical phenotype of a patient, a standard mtDNA analysis is performed first. This includes screening for common mtDNA point mutations (m.3243A $>\mathrm{G}, \quad \mathrm{m} .8344 \mathrm{~A}>\mathrm{G}, \quad \mathrm{m} .8993 \mathrm{~T}>\mathrm{G} / \mathrm{C}$ and $\mathrm{LHON}$ mutations) in blood using restriction fragment length polymorphism (RFLP) analysis or pyrosequencing, which allow for the accurate quantification of heteroplasmy levels. Furthermore, the presence of mtDNA deletions in blood or muscle can be detected with long range PCR. The mtDNA copy number is determined by quantitave PCR (QPCR) and is represented by the ratio of the number of copies of a mtDNA-encoded gene (e.g. 12S $r R N A)$ and the number of copies of a nDNA-encoded gene (e.g. RNAP). A decrease of the copy number below $35 \%$ of the copy number of age matched controls is called mtDNA depletion [24]. The detection of mtDNA depletion by QPCR and multiple mtDNA deletions by long range PCR warrant the screening of nuclear genes associated with mtDNA replication and/or the clinical phenotype. When the initial mtDNA analysis is negative, the whole mtDNA genome will be analyzed e.g. using the Affymetrix mtDNA resequencing chips (MitoChip), preferably in muscle samples. mtDNA variants detected by this method are usually confirmed by conventional Sanger sequencing. In the absence of pathogenic mtDNA mutations or based on the clinical phenotype, nuclear OXPHOS genes are being screened. In case the phenotype resembles that of patients for which a pathogenic mutation has been described previously (e.g. POLG1, ANT), the corresponding gene is screened by direct sequencing of the exonic regions. When no good candidate gene can be found in literature and multiple individuals of a family present a similar phenotype, linkage (single nucleotide polymorphism (SNP)) analysis can be performed to identify new candidate genes. Usually, this results in multiple candidate genes which are screened for mutations by direct sequencing. Recently, exome sequencing is used on itself or in combination with linkage studies to find pathogenic DNA variations in a high throughput manner [25]. Once a pathogenic mutation (mtDNA or nDNA) is identified, family members of the index patient will be examined and for mtDNA mutations, accurate heteroplasmy levels will be determined.

\section{Pathophysiology}

The clinical manifestation of OXPHOS disorders can occur immediately at birth, deteriorate progressively during life or be triggered by a number of events. These triggers of full-blown OXPHOS disease include viral infections [26, 27] and adverse effects of drugs, e.g. valproic acid induced liver failure in patients with mutations in POLG1 [28], mtDNA depletion due to nucleotide reverse transcriptase inhibitors (NRTIs) for the treatment of human immunodeficiency virus (HIV) [29]).

There are a number of approaches to study the pathophysiology of OXPHOS disorders. In the past, pathophysiology was mainly studied in a 'hypothesis-driven' manner. A hypothesis was posed based on existing incomplete knowledge and tested in carefully designed experiments. Accordingly, the hypothesis could be falsified and rejected or supported by the data. At present, the use of 'discovery-driven' research is emphasized. This includes a systems biology approach, studying the interactions of 
large numbers of molecules (DNA, mRNAs or proteins) that constitute the biological system and using data mining to generate (new) hypotheses by induction. High-density measurement technologies such as whole genome gene expression microarrays, high performance liquid chromatography and mass spectrometry are indispensible for this approach. Ultimately, the new hypotheses and theories that arise inductively should be tested in a 'hypothesis-driven' manner to lead to valid knowledge [30]. Finally, animal models have been very useful to study pathophysiology in vivo and to examine the effect of potential therapeutic or adverse drugs [31-35].

So far, research on OXPHOS pathogenesis has led to the identification of a number of biological processes that are assumed to play a role in OXPHOS disorders. Altogether, a dysfunctional OXPHOS system can increase the production of ROS [22, 36], can lead to abnormal mitochondrial fission/fusion [37] and a disturbed mitochondrial membrane potential [38] and will be unable to supply sufficient ATP to meet cellular needs (figure 1.4). Part of this ATP is necessary to fuel cellular import and export of key metabolites and inorganic ions such as calcium [39]. Eventually, increased oxidative stress, a dysfunctional mitochondrial membrane potential and impaired calcium homeostasis can culminate in apoptosis [40] (figure 1.4). In general, changes in oxidative stress and apoptosis co-exist with a deficiency of at least one OXPHOS complex [41-44], but the OXPHOS deficiency is not required for their involvement in the pathogenesis [45].

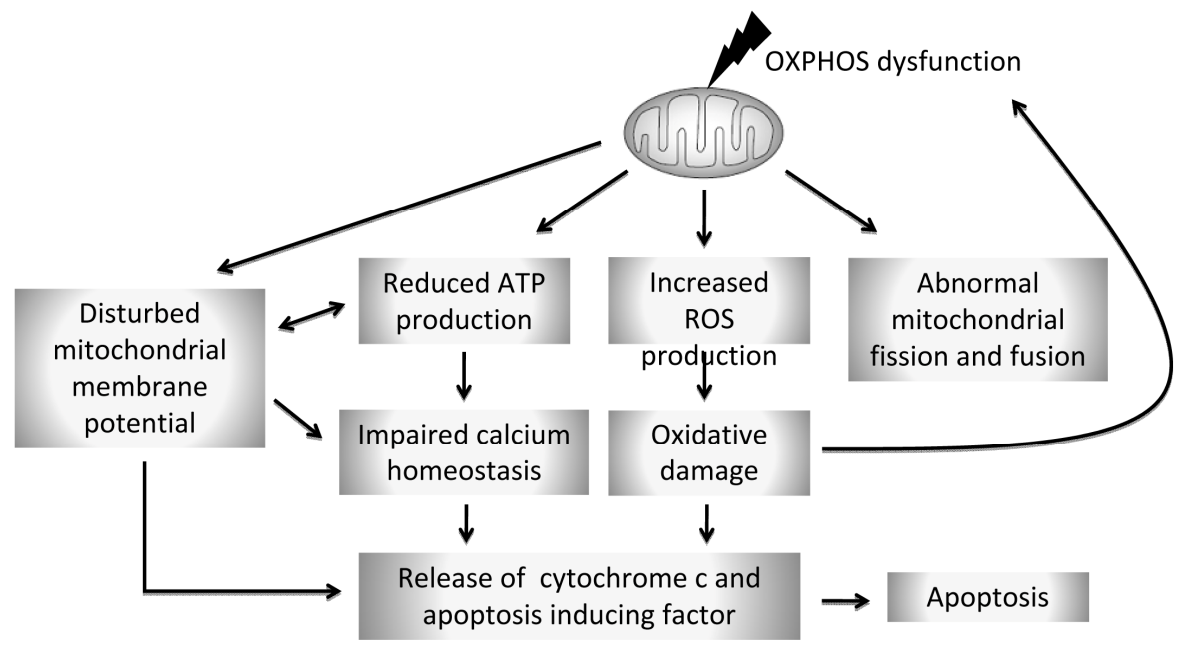

Figure 1.4. Physiopathological processes involved in OXPHOS disease.

\section{Oxidative stress}

Although ROS are generated by multiple other enzymes and processes in a cell, e.g. NADPH oxidases and lipid metabolism, it has been estimated that mitochondria produce most ( 90\%) of cellular ROS at complexes I and III [46]. In the process of oxidative phosphorylation, oxygen is consumed for the production of ATP. In physiological conditions, approximately $0.2 \%$ of the total consumed oxygen is 
converted to ROS (superoxide anion and hydrogen peroxide). Any disturbance of the electron transport chain can increase the production of ROS [46]. Previously, ROS were considered to be merely harmful because of their capacity to oxidize DNA, lipids, carbohydrates and proteins [47]. Oxidation of these macromolecules will lead to mutagenesis [48], diminished functioning [49, 50] and loss of structural integrity [49]. However, ROS per se are not always a negative given. Nowadays, ROS are also believed to play a role as redox messengers in several signaling pathways leading to cellular adaptation, e.g. muscle fiber adaptation in response to both increased contractile activity and prolonged periods of muscle disuse [51].

To keep a balance between its role in signaling and toxic effects, ROS levels are tightly monitored and regulated by the antioxidant defense system. An overview of the most familiar contributors to the mitochondrial antioxidant defense system is depicted in figure 1.5. Superoxide $\left(\mathrm{O}_{2}^{-{ }^{-}}\right)$produced at sites from complex I and III will be dismutated to hydrogen peroxide $\left(\mathrm{H}_{2} \mathrm{O}_{2}\right)$ by superoxide dismutase (SOD) in the mitochondrial matrix (Manganese (Mn) SOD) or cytosol (Copper/Zinc (Cu/Zn) SOD). Hydrogen peroxide can be converted by catalase (CAT) in the cytosol and the glutathione and thioredoxin detoxification systems in the mitochondrial matrix. Hydrogen peroxide that is not converted to water can lead to the formation of highly reactive hydroxyl radicals $(\mathrm{OH})$ through the Fenton reaction.

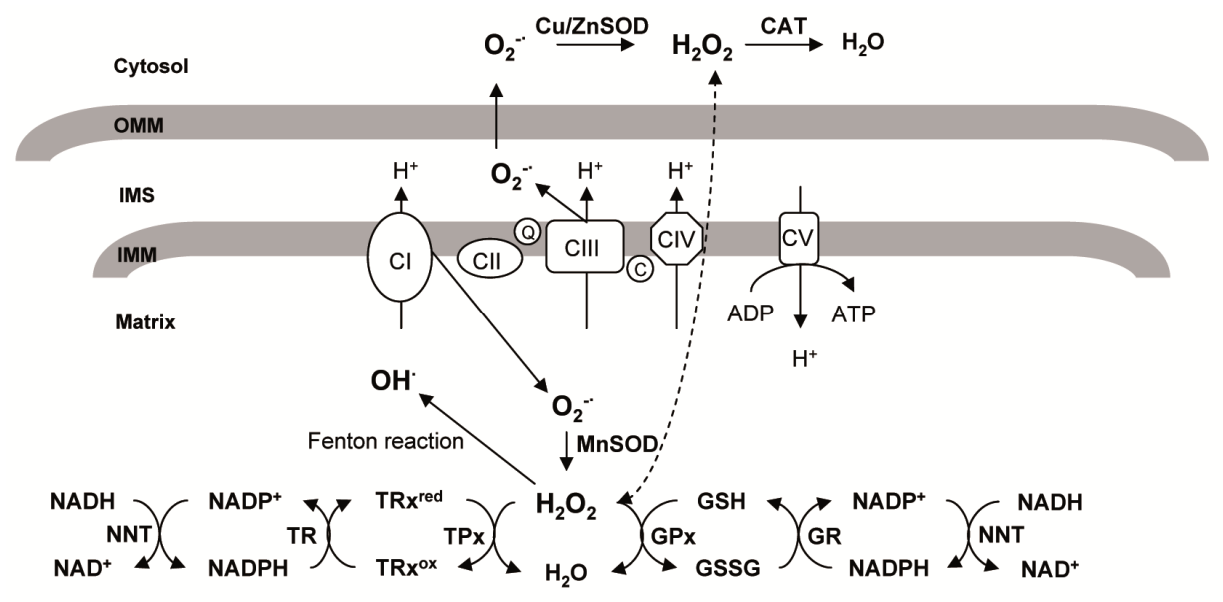

Figure 1.5. Mitochondrial antioxidant defense system. $\mathrm{OMM}=$ outer mitochondrial membrane, IMS = inner membrane space, IMM = inner mitochondrial membrane, GSH = reduced glutathione, GSSG = oxidized glutathione, GPx = glutathione peroxidase, GR = glutathione reductase, NNT = nicotinamide nucleotide transhydrogenase, $T R x^{\text {red }} / T_{R x}{ }^{\text {ox }}=$ reduced and oxidized thioredoxin, respectively, TPx = thioredoxin peroxidase, $\mathrm{TR}=$ thioredoxin reductase.

Since OXPHOS disorders are characterized by disturbances in the electron transport chain, various studies have examined the role of oxidative stress in their pathogenesis (table 1.2). Although some addressed the question whether or not oxidative stress is actively involved in causing disease phenotypes, their results do not always 
correspond. Several reasons can be given for this. First of all, some studies examined only two or three similar oxidative stress parameters. In order to get an impression of the oxidative status of a cell or tissue, three categories of parameters, i.e. ROS levels, antioxidant system levels and oxidative damage levels, should be documented. Especially the last read-out will give valuable information on how patients suffer from the consequences of oxidative stress. Another problem for comparing different experiments is the variety of protocols to measure one specific parameter, e.g. DNA oxidation can be determined by HPLC, ELISA, mass spectrometry, western blot and immunohistochemistry, each with its own advantages, disadvantages and limitations. Measurements with different protocols do not always give comparable results [47]. Finally, the examined tissue and the small sample size of the cohorts can also contribute to conflicting results. Conclusively, as can be appreciated from table 1.2, some OXPHOS disorders (MELAS, MERRF and NARP) show more evidence for the presence of oxidative stress and concomitant damage than others (POLG1 and SURF1 mutations). As there is no consensus, it is recommended to analyze a number of carefully chosen stress parameters, as indicated above, in parallel using the same methods in different OXPHOS disorders [52]. As a result, one will be able to compare the involvement of oxidative stress in each of those diseases and thus evaluate the relevance of antioxidant therapy.

\footnotetext{
Table 1.2 legend. The involvement of oxidative stress parameters (production, defense and damage) in different OXPHOS disorders as documented in literature.

$\uparrow=$ increased, $\downarrow=$ decreased, $N=$ normal, MnSOD = mitochondrial superoxide dismutase, CuZnSOD = cytosolic superoxide dismutase, $\mathrm{GPx}=$ glutathione peroxidase, $\mathrm{GR}=$ glutathione reductase, $\mathrm{GST}=$ glutathione $S$ transferase, $\mathrm{GSSG}=$ oxidized glutathione, $\mathrm{GSH}=$ reduced glutathione, $8-\mathrm{OHdG}=8-$ hydroxy-2deoxy guanosine, 4-HNE = 4-hydroxynonenal, MDA = malondialdehyde

${ }^{+} \mathrm{F}=$ fibroblasts, $\mathrm{M}=$ muscle, $\mathrm{C}=$ cybrids, $\mathrm{BI}=$ blood, $\mathrm{L}=$ lymfocytes, $\mathrm{My}=$ myoblasts, $\mathrm{Br}=$ brain, $\mathrm{Mi}=$ mice

${ }^{*} \mathrm{p}=$ number of patients, $\mathrm{m}=$ number of different mutations, $\mathrm{cl}=$ number of cell lines with different heteroplasmy percentage
} 


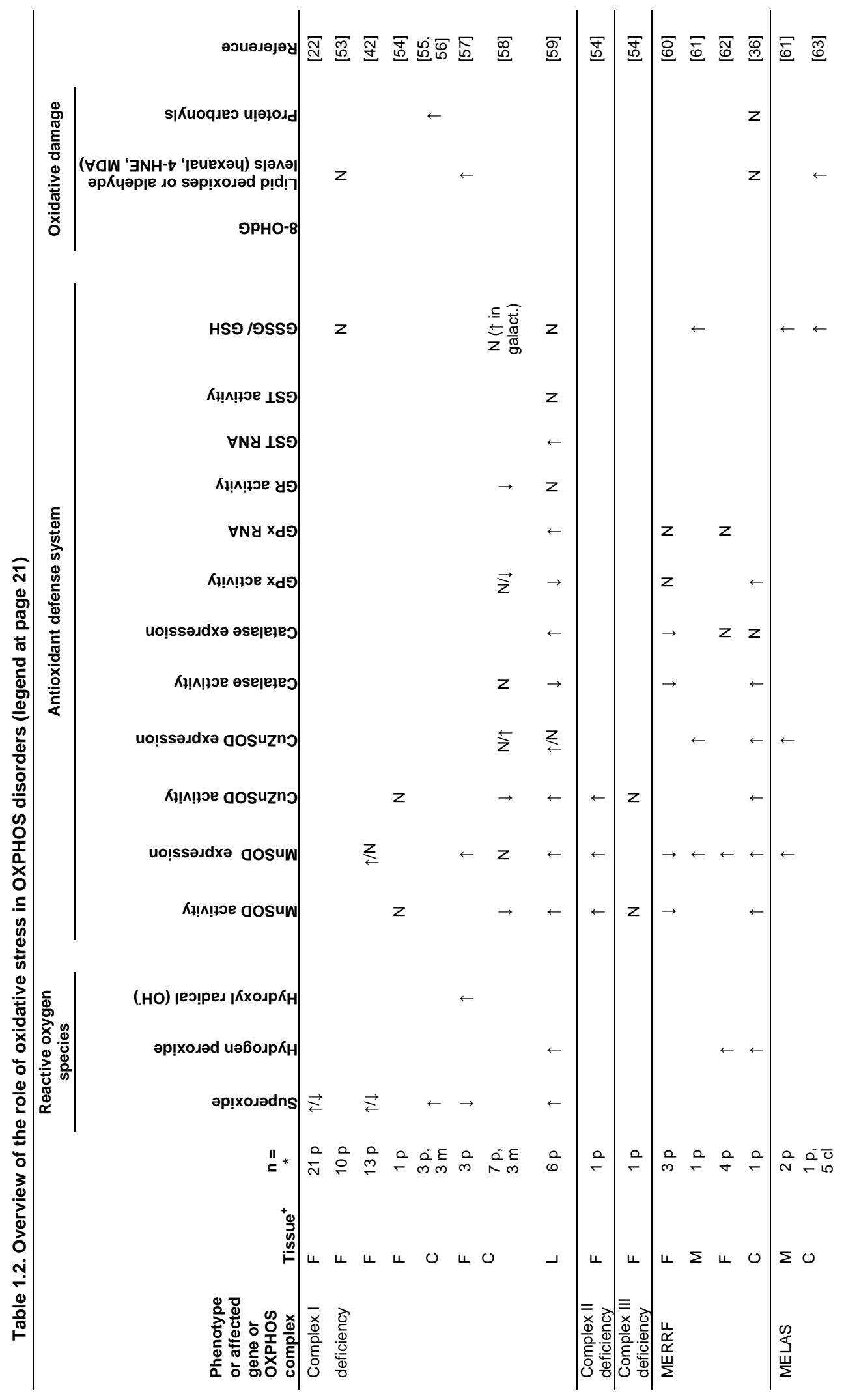




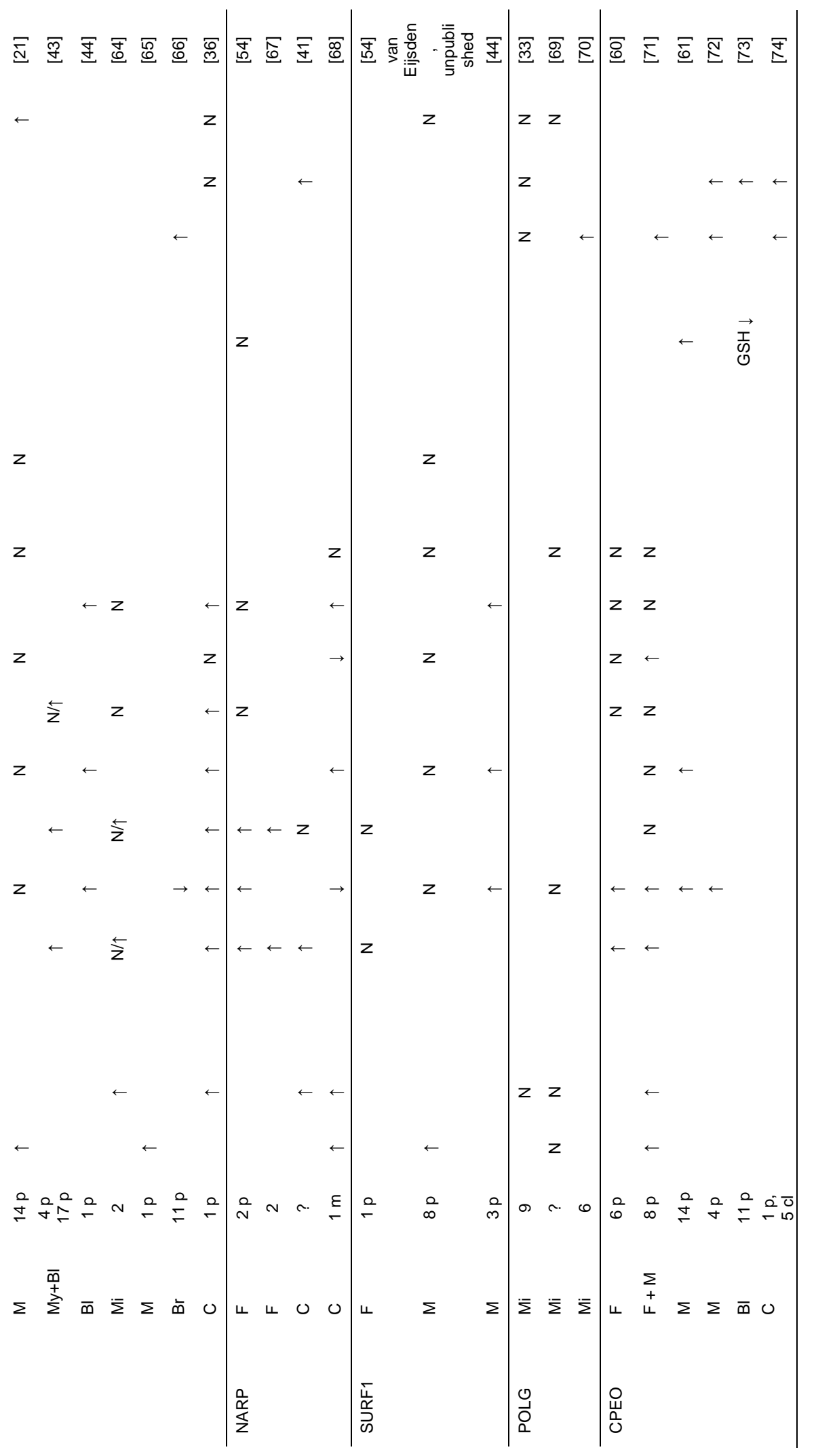




\section{Apoptosis}

Mitochondria are at the crossroads of apoptosis (programmed cell death) regulation. The intrinsic mitochondrial apoptosis pathway can be initiated by a number of signals including DNA damage, growth factor deprivation and oxidative stress [75]. In short, mitochondrial outer membrane permeabilization leads to the release of soluble mitochondrial intramembrane proteins, such as cytochrome c, that trigger the proteolytic caspase cascade and cause inactivation of the electron transport chain [76]. Multiple studies have shown the involvement of apoptosis in muscle of patients with OXPHOS disorders (PEO [72, 77, 78], MELAS [45, 72, 78], MERRF [45, 78] and KSS [77]). However, patients with comparable phenotypes did not show equal signs of apoptosis in other studies [79-81]. Apoptosis was shown to be the central mechanism of tissue dysfunction in a mtDNA mutator mouse model (POLG1 D257A knock-in) [33], but embryonic fibroblasts from transgenic mice with the same mutation were not more susceptible to undergo oxidative stress induced apoptosis than wild type mouse embryonic fibroblasts [69].

Altogether, the current results on the role of apoptosis in OXPHOS disorders are not straightforward and point to a dysregulation of apoptotic decisions rather than increased apoptosis on itself. One theory could be that damaged cells which should die, sometimes fail herein whereas some healthy cells become apoptotic without valid reason. This compromises physiological functions and could also favor replicative senescence [82].

\section{Examples of pathophysiological models for OXPHOS disorders}

In the next three paragraphs, three of the most frequent OXPHOS disorders will be described in more detail, including clinical phenotype, genetic cause and recent research on the molecular processes involved. Patients with isolated complex I (Cl) deficiency, mitochondrial encephalomyopathy, lactic acidosis and stroke-like episodes (MELAS) patients and polymerase gamma (POLG1) patients represent three different disease classes i.e. defects in OXPHOS enzyme activity, mitochondrial protein translation due to a tRNA mutation and mtDNA maintenance, respectively. As a relatively high number of patients presents with these disorders, it is relevant to study their pathophysiology in order to improve their treatment options.

\section{Isolated $\mathrm{Cl}$ deficiency}

$\mathrm{Cl}$ deficiency leads to a number of clinical features including basal ganglia and/or brainstem lesions, respiratory abnormalities, muscular hypotonia, failure to thrive, seizures and lactic academia [83]. Most patients have a normal prenatal development and birth, but will develop symptoms within their first year of life, which are often fatal [38], or less severe symptoms later in life (e.g. Leber hereditary optic neuropathy [LHON]). $\mathrm{Cl}$ deficiency is diagnosed when $\mathrm{Cl}$ activity in muscle or skin biopsies is decreased compared to control specimen. Decreased $\mathrm{Cl}$ activity can be caused by 
mutations in both the mtDNA and nuclear genes, but often the genetic mutation leading to the deficiency cannot be identified [84].

LHON (table 1.1) is associated with multiple mtDNA mutations, but those most frequent are m.3460G $>A, m .11778 \mathrm{G}>\mathrm{A}$ and m.14484T $>\mathrm{C}$ in the ND1, ND4 and ND6 genes of $\mathrm{Cl}$, respectively [85]. LHON mutations are usually inherited and homoplasmic [86]. In mutant LHON cells $\mathrm{Cl}$ redox activity has been shown to be normal or reduced [87, 88], but the interaction between $\mathrm{Cl}$ and its ubiquinone substrate (CoQ) might have been affected by the three LHON mutations. This led to a decreased $\mathrm{Cl}$ dependent ATP synthesis and the overproduction of ROS [85].

In case nuclear genes are involved, the reduced $\mathrm{Cl}$ activity is primarily caused by lower levels of normal functioning $\mathrm{Cl}$ instead of a decrease in intrinsic activity of the complex [89]. In resting fibroblasts of $\mathrm{Cl}$ deficient patients, the overall cellular ATP levels were normal, which suggests that in the normal situation, ATP derived from glycolysis and/or residual $\mathrm{Cl}$ activity is sufficient in fibroblasts. However, the same study showed that the reduced $\mathrm{Cl}$ activity led to a depolarization of the mitochondrial membrane potential and reduced $\mathrm{Ca}^{+}$content in the endoplasmic reticulum (ER). The authors hypothesized that the ATP supply to the ER $\mathrm{Ca}^{+}$(SERCA) pumps was insufficient to maintain normal ER $\mathrm{Ca}^{+}$levels which probably influences cellular calcium homeostasis [39]. Furthermore, in $\mathrm{Cl}$ patients it has been shown that residual $\mathrm{Cl}$ activity was inversely correlated with the production of ROS (superoxide and superoxide-derived ROS) [22]. Notably, whenever a certain threshold of residual $\mathrm{Cl}$ activity (and thus ROS production) is passed, mitochondrial morphology changed. In fibroblast cell lines with a relatively 'mild' $\mathrm{Cl}$ deficiency, mitochondrial morphology was normal or even more elongated, whereas patient cell lines with a severe $\mathrm{Cl}$ deficiency (and thus higher ROS levels) showed a fragmented mitochondrial morphology. These differences probably coincide with a decreased probability to compensate for increased ROS levels or ROS related damage [38]. An unbiased approach, such as gene expression analysis, will help integrating the current knowledge into a pathophysiological model that might be able to distinguish cause from effect.

\section{MELAS}

Although tRNA sequences comprise only a small fraction $(\sim 10 \%)$ of the mtDNA, more than half of the known pathogenic mtDNA mutations are found within the tRNA genes (MITOMAP database; www.mitomap.org/MITOMAP). The MELAS syndrome is the most common mitochondrial disorder and in $80 \%$ of the cases, patients carry the m.3243A>G mutation in the tRNA Leu(UUR) gene [90]. Clinical features associated with MELAS include recurrent strokes, CPEO, retinitis pigmentosa, optic atrophy deafness, dementia, extrapyramidal features ataxia epilepsy, myoclonus myopathy, cardiomyopathy, neuropathy, diabetes mellitus, gastrointestinal features (malabsorption and/or dysmotility), short stature and lipomata [11]. In a large patient cohort, a correlation could be observed between clinical features which occurred in $>15 \%$ of the cases and the level of mutant mtDNA in muscle $(n=111)$ but not in blood $(n=73)$. A positive correlation with mutation percentage was identified for the frequency of recurrent strokes, dementia, epilepsy and ataxia, whereas an inverse correlation was detected for CPEO myopathy and deafness [11]. Another study could not find a 
correlation between the percentage of mutated mtDNA and patient characteristics, which could be due to the smaller sample size $(n=22)$. However, they did find a significant inverse correlation between the m.3243A $>$ G mutant load and age of onset and $\mathrm{Cl}$ activity [91]. Additionally, the m.3243A $>\mathrm{G}$ mutation percentage was positively correlated with hyperglycemia in diabetic patients [92].

The m.3243A $>G$ mutation leads to incorrect tRNA processing and enzyme maturation due to disruption of the tertiary tRNA structure. Furthermore, the m.3243A>G mutation impairs taurine modification of the anticodon wobble position which is responsible for precise and efficient codon recognition [93, 94]. Eventually, mitochondrial translation is impaired because of a decreased steady-state of the normal aminoacylated tRNA [95]. The decreased protein synthesis rate will cause decreased enzyme activity and severe respiratory defects [90]. A gene expression study in muscle of symptomatic and asymptomatic mutation carriers identified increased protein turnover, oxidative stress, apoptosis and complement activation [21]. In a-symptomatic carriers, the amount of protein regeneration and OXPHOS stimulation seemed sufficient to prevent the occurrence of symptoms. However, in symptomatic carriers, excessive protein damage presumably leads to termination of repair/adaptive processes and a switch to muscle regeneration, indicated by stronger complement activation [21]. A more in depth analysis of the oxidative stress (production, scavenging and damage) might be able to fine-tune the current model of MELAS pathogenesis.

\section{POLG1 patients}

DNA polymerase gamma (pol $\mathrm{\gamma}$ ) is the only DNA polymerase involved in maintenance of the mtDNA and consists of a catalytic subunit and a homodimeric accessory subunit, encoded by the POLG1 and POLG2 genes, respectively [96, 97]. Over 150 mutations, which can be of a dominant or a recessive nature, have been detected in the POLG1 gene (Human DNA Polymerase Gamma Mutation Database http://tools.niehs.nih.gov/polg/). Only recently, Euro and colleagues were able to cluster the recessive mutations into five distinct modules based on the recently solved crystal structure of the enzyme [98]. Mutations in POLG1 are associated with a series of disorders such as PEO (table 1.1), Alpers-Huttenlocher syndrome, childhood myocerebrohepatopathy spectrum (MHCS) disorders, ataxia neuropathy spectrum (ANS) disorders, myoclonus epilepsy myopathy sensory ataxia (MEMSA) and sensory ataxic neuropathy with dysarthria and ophtalmoplegia (SANDO) [99, 100]. Additionally, POLG1 mutations have been found to play a role in nucleoside reverse-transcriptase inhibitor (NRTI) [101] and valproic acid [28] toxicity and premature ovarian failure (POF) [102, 103].

Mutations in the exonuclease and polymerase domain of POLG1 or combinations are associated with decreased mtDNA copy number in liver, muscle and fibroblasts of POLG1 patients [104]. Decreased nucleotide insertion specificity [105], decreased catalytic activity [105], loss of interaction between the catalytic and accessory subunits [106] due to POLG1 mutations have been observed in vitro. Furthermore, the accumulation of heteroplasmic mtDNA point mutations, which can [107] or cannot [108] stimulate mtDNA deletion formation, in skeletal muscle $[107,108]$ and cultured human cells expressing POLG1 fusion proteins [109] have been reported. Whereas complete 
knockout mice died prenatally [110], transgenic proofreading deficient mouse models (D257A POLG1 knock-in) showed accelerated ageing phenotypes with increased levels of mtDNA point mutations without evidence for the involvement of oxidative stress [33, 34]. Human studies are limited to in vitro characterization of purified or recombinant enzymes [111], in vivo analysis of the functions of the pol y protein and its domains in cultured cells [109] and large scale screening of mtDNA molecules in skeletal muscle and fibroblasts [104, 107, 108]. These studies point out a higher probability of accumulating mtDNA mutations and/or depleting mtDNA when POLG1 is mutated. In addition, deficiencies in respiratory chain enzymes have been described in muscle of POLG1 patients [112]. Up to now, the more integrative studies of POLG pathogenesis are performed solely in mouse. An unbiased top-down approach (e.g. whole genome gene expression) may help elucidating processes involved in human POLG pathogenesis. The obtained results will indicate if the same disease mechanisms apply for rodents and human beings.

\section{Therapy in OXPHOS disorders}

Although knowledge on OXPHOS disorders and their underlying pathological mechanisms is emerging, a cure remains unavailable for most of them. Treatment is usually based on preventing complications, minimizing disability (symptoms) and providing prognostic and genetic counseling [113]. Additionally, the use of certain medication might be contra-indicated in some OXPHOS disorders, e.g. valproic acid for the management of epileptic seizures in case of POLG1 mutations [114]. Furthermore, the efficacy of other pharmacological agents is highly anecdotic. Nevertheless, various treatment cocktails of vitamins and cofactors (e.g. riboflavin, thiamine, folic acid, Lcarnitine and creatine monohydrate, coenzyme Q10) are administrated to patients with OXPHOS disease because they are presumed harmless [114].

Recently, exercise-based therapies were tested for the treatment of OXPHOS disorders [115]. On the one hand, endurance training was shown to improve oxidative capacity in patients with mtDNA mutations but did not decrease the percentage of mutated mtDNA (so-called 'gene shifting') [116-118]. On the other hand, resistance training lead to muscle regeneration through the activation of satellite cells which did not harbor the mutant mtDNA [17]. After muscle regeneration, less muscle fibers contained the mutant mtDNA and initial studies showed improved biochemical activity [119, 120]. These preliminary results are encouraging for minimizing disability in patients with OXPHOS disorders.

\section{Aim and outline of this thesis}

The components of the OXPHOS system are encoded by both the mitochondrial and nuclear DNA. Mutations in genes encoding structural OXPHOS elements or factors involved in the maintenance or assembly of the OXPHOS system lead to a number of 
diseases, in general referred to as OXPHOS disorders. The underlying pathological mechanisms of these diseases are not entirely understood and there is a lack of efficient therapies. The central aim of this thesis is to study the pathophysiology of a number of specific OXPHOS disorders and to create new models and identify new potential targets for therapeutic interventions. Therefore, microarray gene expression profiling was applied as an unbiased approach to find clues for biological processes that are affected by the individual diseases and that can be monitored or targeted by new therapeutic strategies. Additionally, oxidative stress has generally been considered to be an important pathogenic factor in OXPHOS disorders although the efficacy of antioxidant therapy is highly anecdotic. Hence, the involvement and extent of oxidative stress in a number of different OXPHOS disorders was interrogated. Characterized cell lines can be used as model systems to test compounds that have potential to ameliorate or slow down progression of the disease.

Altogether, the aim of this thesis is to create models or approaches to:

- Facilitate diagnostics of mtDNA-based OXPHOS disorders by determining the non-pathogenic variation of mtDNA in the general population.

- Gain knowledge on primary and secondary biological processes involved in OXPHOS disorders which have an impact on prognosis and therapy.

- Explore the extent to which different pathogenic mtDNA and nuclear DNA mutations induce oxidative stress and the consequences of this.

Chapter 2 describes the analysis of the mtDNA of 730 European subjects and presents novel data regarding the distribution of homoplasmic variants across the mtDNA. This comprehensive overview of mtDNA polymorphisms distinguishes between regions and positions which are likely not critical, pathogenic mutations targeting mainly conserved regions and regions containing no mutations at all. These data provide valuable information for evaluating the pathogenicity of mtDNA variants and counseling families. To improve prognosis and therapy options after genetic diagnosis, molecular disease pathways were identified in chapter 3 and 4 using microarray gene expression profiles in skeletal muscle of POLG1 patients and fibroblast cell lines of complex I patients, respectively. Both chapters indicated a role of oxidative stress in OXPHOS pathology. Therefore, chapter 5 evaluated the effect of pathogenic mtDNA mutations (in ND1, ND5 and tRNALeu) and mtDNA maintenance defects (POLG1 mutations) on oxidative stress. This resulted in disease and patient specific signatures that indicate which parameter should best be used, i.e. oxidative damage, to evaluate which patients might benefit from antioxidant therapy. The cell line models of chapter 4 and 5 are now available for preliminary testing of the therapeutic effect of small compounds. The relevance of the results presented in chapter 2 to 5 in respect to the development of therapeutic strategies for OXPHOS disorders will be discussed in chapter 6 . 


\section{References}

1. Schaefer, A.M., et al., The epidemiology of mitochondrial disorders--past, present and future. Biochim Biophys Acta, 2004. 1659(2-3): p. 115-20.

2. Wallace, D.C., Mitochondrial DNA mutations in disease and aging. Environ Mol Mutagen, 2010. 51(5): p. 440-50.

3. Rossignol, R., et al., Tissue variation in the control of oxidative phosphorylation: implication for mitochondrial diseases. Biochem J, 2000. 347 Pt 1: p. 45-53.

4. Montoya, J., et al., Diseases of the human mitochondrial oxidative phosphorylation system. Adv Exp Med Biol, 2009. 652: p. 47-67.

5. Dimauro, S. and P. Rustin, A critical approach to the therapy of mitochondrial respiratory chain and oxidative phosphorylation diseases. Biochim Biophys Acta, 2009. 1792(12): p. 1159-67.

6. Jacobs, J.A.M. The transmission and segregation of mitochondrial DNA mutations [PhD thesis]. Maastricht: Maastricht University; 2007.

7. Wanrooij, S. and M. Falkenberg, The human mitochondrial replication fork in health and disease. Biochim Biophys Acta, 2010. 1797(8): p. 1378-88.

8. Pitchon, E.M., et al., Patient with Fanconi Syndrome (FS) and retinitis pigmentosa $(R P)$ caused by a deletion and duplication of mitochondrial DNA (mtDNA). Klin Monbl Augenheilkd, 2007. 224(4): p. 340-3.

9. Liu, C.S., et al., Alteration in the copy number of mitochondrial DNA in leukocytes of patients with mitochondrial encephalomyopathies. Acta Neurol Scand, 2006. 113(5): p. 334-41.

10. Carelli, V., C. Giordano, and G. d'Amati, Pathogenic expression of homoplasmic $m t D N A$ mutations needs a complex nuclear-mitochondrial interaction. Trends Genet, 2003. 19(5): p. 257-62.

11. Chinnery, P.F., et al., Molecular pathology of MELAS and MERRF. The relationship between mutation load and clinical phenotypes. Brain, 1997. 120 ( Pt 10): p. 171321.

12. Tuppen, H.A., et al., Mitochondrial DNA mutations and human disease. Biochim Biophys Acta, 2010. 1797(2): p. 113-28.

13. Yarham, J.W., et al., Mitochondrial tRNA mutations and disease. Wiley Interdiscip Rev RNA, 2010. 1(2): p. 304-24.

14. Montoya, J., et al., 20 years of human mtDNA pathologic point mutations: carefully reading the pathogenicity criteria. Biochim Biophys Acta, 2009. 1787(5): p. 476-83.

15. Zeviani, M., A. Spinazzola, and V. Carelli, Nuclear genes in mitochondrial disorders. Curr Opin Genet Dev, 2003. 13(3): p. 262-70.

16. Janssen, A.J., J.A. Smeitink, and L.P. van den Heuvel, Some practical aspects of providing a diagnostic service for respiratory chain defects. Ann Clin Biochem, 2003. 40(Pt 1): p. 3-8.

17. Rahman, S. and M.G. Hanna, Diagnosis and therapy in neuromuscular disorders: diagnosis and new treatments in mitochondrial diseases. $\mathrm{J}$ Neurol Neurosurg Psychiatry, 2009. 80(9): p. 943-53.

18. Chretien, D. and P. Rustin, Mitochondrial oxidative phosphorylation: pitfalls and tips in measuring and interpreting enzyme activities. J Inherit Metab Dis, 2003. 26(2-3): p. 189-98.

19. Kim, S.H., et al., Mutations of ACADS gene associated with short-chain acylcoenzyme A dehydrogenase deficiency. Ann Clin Lab Sci, 2011. 41(1): p. 84-8.

20. Passarelli, C., et al., GSSG-mediated Complex I defect in isolated cardiac mitochondria. Int J Mol Med, 2010. 26(1): p. 95-9.

21. van Eijsden, R.G., et al., Termination of damaged protein repair defines the occurrence of symptoms in carriers of the m.3243A > G tRNA(Leu) mutation. J Med Genet, 2008. 45(8): p. 525-34. 
22. Verkaart, S., et al., Superoxide production is inversely related to complex I activity in inherited complex I deficiency. Biochim Biophys Acta, 2007. 1772(3): p. 373-81.

23. Murray, J., et al., Monitoring oxidative and nitrative modification of cellular proteins; a paradigm for identifying key disease related markers of oxidative stress. Adv Drug Deliv Rev, 2008. 60(13-14): p. 1497-503.

24. Nguyen, K.V., et al., Molecular diagnosis of Alpers syndrome. J Hepatol, 2006. 45(1): p. 108-16.

25. Ng, S.B., et al., Massively parallel sequencing and rare disease. Hum Mol Genet, 2010. 19(R2): p. R119-24.

26. Smeitink, J. and L. van den Heuvel, Human mitochondrial complex I in health and disease. Am J Hum Genet, 1999. 64(6): p. 1505-10.

27. Hoefs, S.J., et al., NDUFA2 complex I mutation leads to Leigh disease. Am J Hum Genet, 2008. 82(6): p. 1306-15.

28. Saneto, R.P., et al., POLG DNA testing as an emerging standard of care before instituting valproic acid therapy for pediatric seizure disorders. Seizure, 2010. 19(3): p. 140-6.

29. Stankov, M.V., et al., Mitochondrial DNA depletion and respiratory chain activity in primary human subcutaneous adipocytes treated with nucleoside analogue reverse transcriptase inhibitors. Antimicrob Agents Chemother, 2010. 54(1): p. 280-7.

30. van Beek, J.H., Data integration and analysis for medical systems biology. Comp Funct Genomics, 2004. 5(2): p. 201-4.

31. Koene, S., et al., Mouse models for nuclear DNA-encoded mitochondrial complex I deficiency. J Inherit Metab Dis, 2010.

32. Fernandez-Ayala, D.J., et al., Gene expression in a Drosophila model of mitochondrial disease. PLoS One, 2010. 5(1): p. e8549.

33. Kujoth, G.C., et al., Mitochondrial DNA mutations, oxidative stress, and apoptosis in mammalian aging. Science, 2005. 309(5733): p. 481-4.

34. Trifunovic, A., et al., Premature ageing in mice expressing defective mitochondrial DNA polymerase. Nature, 2004. 429(6990): p. 417-23.

35. Torraco, A., et al., Mouse models of oxidative phosphorylation defects: powerful tools to study the pathobiology of mitochondrial diseases. Biochim Biophys Acta, 2009. 1793(1): p. 171-80.

36. Vives-Bauza, C., et al., Enhanced ROS production and antioxidant defenses in cybrids harbouring mutations in mtDNA. Neurosci Lett, 2006. 391(3): p. 136-41.

37. Schon, E.A., et al., Therapeutic prospects for mitochondrial disease. Trends Mol Med, 2010. 16(6): p. 268-76.

38. Distelmaier, F., et al., Mitochondrial complex I deficiency: from organelle dysfunction to clinical disease. Brain, 2009. 132(Pt 4): p. 833-42.

39. Willems, P.H., et al., Mitochondrial Ca2+ homeostasis in human NADH:ubiquinone oxidoreductase deficiency. Cell Calcium, 2008. 44(1): p. 123-33.

40. Norenberg, M.D. and K.V. Rao, The mitochondrial permeability transition in neurologic disease. Neurochem Int, 2007. 50(7-8): p. 983-97.

41. Mattiazzi, M., et al., The mtDNA T8993G (NARP) mutation results in an impairment of oxidative phosphorylation that can be improved by antioxidants. Hum Mol Genet, 2004. 13(8): p. 869-79.

42. Pitkanen, S. and B.H. Robinson, Mitochondrial complex I deficiency leads to increased production of superoxide radicals and induction of superoxide dismutase. $\mathrm{J}$ Clin Invest, 1996. 98(2): p. 345-51.

43. Rusanen, H., K. Majamaa, and I.E. Hassinen, Increased activities of antioxidant enzymes and decreased ATP concentration in cultured myoblasts with the 3243A-$>$ G mutation in mitochondrial DNA. Biochim Biophys Acta, 2000. 1500(1): p. 10-6.

44. Di Giovanni, S., et al., Apoptosis and ROS detoxification enzymes correlate with cytochrome $c$ oxidase deficiency in mitochondrial encephalomyopathies. Mol Cell Neurosci, 2001. 17(4): p. 696-705. 
45. Mirabella, M., et al., Apoptosis in mitochondrial encephalomyopathies with mitochondrial DNA mutations: a potential pathogenic mechanism. Brain, 2000. 123 ( Pt 1): p. 93-104.

46. Balaban, R.S., S. Nemoto, and T. Finkel, Mitochondria, oxidants, and aging. Cell, 2005. 120(4): p. 483-95.

47. Hawkins, C.L., P.E. Morgan, and M.J. Davies, Quantification of protein modification by oxidants. Free Radic Biol Med, 2009. 46(8): p. 965-88.

48. Sekiguchi, M. and T. Tsuzuki, Oxidative nucleotide damage: consequences and prevention. Oncogene, 2002. 21(58): p. 8895-904.

49. Bayeva, M. and H. Ardehali, Mitochondrial dysfunction and oxidative damage to sarcomeric proteins. Curr Hypertens Rep, 2010. 12(6): p. 426-32.

50. Stark, G., Functional consequences of oxidative membrane damage. J Membr Biol, 2005. 205(1): p. 1-16.

51. Powers, S.K., et al., Reactive oxygen species are signalling molecules for skeletal muscle adaptation. Exp Physiol, 2010. 95(1): p. 1-9.

52. Giustarini, D., et al., Oxidative stress and human diseases: Origin, link, measurement, mechanisms, and biomarkers. Crit Rev Clin Lab Sci, 2009. 46(5-6): p. 241-81.

53. Verkaart, S., et al., Mitochondrial and cytosolic thiol redox state are not detectably altered in isolated human NADH:ubiquinone oxidoreductase deficiency. Biochim Biophys Acta, 2007. 1772(9): p. 1041-51.

54. Geromel, V., et al., Superoxide-induced massive apoptosis in cultured skin fibroblasts harboring the neurogenic ataxia retinitis pigmentosa (NARP) mutation in the ATPase6 gene of the mitochondrial DNA. Hum Mol Genet, 2001. 10(11): p. 1221-8.

55. Beretta, S., et al., Leber hereditary optic neuropathy mtDNA mutations disrupt glutamate transport in cybrid cell lines. Brain, 2004. 127(Pt 10): p. 2183-92.

56. Sala, G., et al., Antioxidants partially restore glutamate transport defect in leber hereditary optic neuropathy cybrids. J Neurosci Res, 2008. 86(15): p. 3331-7.

57. Luo, X., et al., Excessive formation of hydroxyl radicals and aldehydic lipid peroxidation products in cultured skin fibroblasts from patients with complex I deficiency. J Clin Invest, 1997. 99(12): p. 2877-82.

58. Floreani, M., et al., Antioxidant defences in cybrids harboring mtDNA mutations associated with Leber's hereditary optic neuropathy. FEBS J, 2005. 272(5): p. 112435.

59. Wani, A.A., et al., Analysis of reactive oxygen species and antioxidant defenses in complex I deficient patients revealed a specific increase in superoxide dismutase activity. Free Radic Res, 2008. 42(5): p. 415-27.

60. Wei, Y.H., et al., Oxidative stress in human aging and mitochondrial diseaseconsequences of defective mitochondrial respiration and impaired antioxidant enzyme system. Chin J Physiol, 2001. 44(1): p. 1-11.

61. Filosto, M., et al., Antioxidant agents have a different expression pattern in muscle fibers of patients with mitochondrial diseases. Acta Neuropathol, 2002. 103(3): p. 215-20.

62. Ma, Y.S., et al., Upregulation of matrix metalloproteinase 1 and disruption of mitochondrial network in skin fibroblasts of patients with MERRF syndrome. Ann N Y Acad Sci, 2005. 1042: p. 55-63.

63. Pang, C.Y., H.C. Lee, and Y.H. Wei, Enhanced oxidative damage in human cells harboring A3243G mutation of mitochondrial DNA: implication of oxidative stress in the pathogenesis of mitochondrial diabetes. Diabetes Res Clin Pract, 2001. 54 Suppl 2: p. S45-56.

64. Li, J., et al., Increased ROS generation and SOD activity in heteroplasmic tissues of transmitochondrial mice with A3243G mitochondrial DNA mutation. Genet Mol Res, 2008. 7(4): p. 1054-62. 
65. Ishikawa, K., et al., Increased reactive oxygen species and anti-oxidative response in mitochondrial cardiomyopathy. Circ J, 2005. 69(5): p. 617-20.

66. Katayama, Y., et al., Accumulation of oxidative stress around the stroke-like lesions of MELAS patients. Mitochondrion, 2009. 9(5): p. 306-13.

67. Dassa, E.P., et al., The mtDNA NARP mutation activates the actin-Nrf2 signaling of antioxidant defenses. Biochem Biophys Res Commun, 2008. 368(3): p. 620-4.

68. Wojewoda, M., J. Duszynski, and J. Szczepanowska, Antioxidant defence systems and generation of reactive oxygen species in osteosarcoma cells with defective mitochondria: Effect of selenium. Biochim Biophys Acta, 2010. 1797(6-7): p. 890-6.

69. Trifunovic, A., et al., Somatic mtDNA mutations cause aging phenotypes without affecting reactive oxygen species production. Proc Natl Acad Sci U S A, 2005. 102(50): p. 17993-8.

70. Lewis, W., et al., Decreased mtDNA, oxidative stress, cardiomyopathy, and death from transgenic cardiac targeted human mutant polymerase gamma. Lab Invest, 2007. 87(4): p. 326-35.

71. Lu, C.Y., et al., Increased expression of manganese-superoxide dismutase in fibroblasts of patients with CPEO syndrome. Mol Genet Metab, 2003. 80(3): p. 321-9.

72. Umaki, Y., et al., Apoptosis-related changes in skeletal muscles of patients with mitochondrial diseases. Acta Neuropathol, 2002. 103(2): p. 163-70.

73. Piccolo, G., et al., Biological markers of oxidative stress in mitochondrial myopathies with progressive external ophthalmoplegia. J Neurol Sci, 1991. 105(1): p. 57-60.

74. Wei, Y.H., et al., Increases of mitochondrial mass and mitochondrial genome in association with enhanced oxidative stress in human cells harboring 4,977 BPdeleted mitochondrial DNA. Ann N Y Acad Sci, 2001. 928: p. 97-112.

75. Jin, Z. and W.S. El-Deiry, Overview of cell death signaling pathways. Cancer Biol Ther, 2005. 4(2): p. 139-63.

76. Green, D.R. and G. Kroemer, The pathophysiology of mitochondrial cell death. Science, 2004. 305(5684): p. 626-9.

77. Monici, M.C., et al., Apoptosis in metabolic myopathies. Neuroreport, 1998. 9(10): p. 2431-5.

78. Ikezoe, K., et al., Apoptosis is suspended in muscle of mitochondrial encephalomyopathies. Acta Neuropathol, 2002. 103(6): p. 531-40.

79. Fagiolari, G., et al., Lack of apoptosis in patients with progressive external ophthalmoplegia and mutated adenine nucleotide translocator-1 gene. Muscle Nerve, 2002. 26(2): p. 265-9.

80. Sciacco, M., et al., Lack of apoptosis in mitochondrial encephalomyopathies. Neurology, 2001. 56(8): p. 1070-4.

81. Otabe, S., et al., Molecular and histological evaluation of pancreata from patients with a mitochondrial gene mutation associated with impaired insulin secretion. Biochem Biophys Res Commun, 1999. 259(1): p. 149-56.

82. Smeitink, J.A., et al., Mitochondrial medicine: a metabolic perspective on the pathology of oxidative phosphorylation disorders. Cell Metab, 2006. 3(1): p. 9-13.

83. Nouws, J., et al., Assembly factors as a new class of disease genes for mitochondrial complex I deficiency: cause, pathology and treatment options. Brain, 2011.

84. Thorburn, D.R., et al., Biochemical and molecular diagnosis of mitochondrial respiratory chain disorders. Biochim Biophys Acta, 2004. 1659(2-3): p. 121-8.

85. Lenaz, G., et al., Bioenergetics of mitochondrial diseases associated with mtDNA mutations. Biochim Biophys Acta, 2004. 1658(1-2): p. 89-94.

86. Wong, L.J., Pathogenic mitochondrial DNA mutations in protein-coding genes. Muscle Nerve, 2007. 36(3): p. 279-93.

87. Carelli, V., et al., Biochemical features of mtDNA 14484 (ND6/M64V) point mutation associated with Leber's hereditary optic neuropathy. Ann Neurol, 1999. 45(3): p. 3208. 
88. Carelli, V., et al., Leber's hereditary optic neuropathy: biochemical effect of 11778/ND4 and 3460/ND1 mutations and correlation with the mitochondrial genotype. Neurology, 1997. 48(6): p. 1623-32.

89. Valsecchi, F., et al., Complex I disorders: causes, mechanisms, and development of treatment strategies at the cellular level. Dev Disabil Res Rev, 2010. 16(2): p. 17582.

90. Finsterer, J., Genetic, pathogenetic, and phenotypic implications of the mitochondrial A3243G tRNALeu(UUR) mutation. Acta Neurol Scand, 2007. 116(1): p. 1-14.

91. Mariotti, C., et al., Genotype to phenotype correlations in mitochondrial encephalomyopathies associated with the A3243G mutation of mitochondrial DNA. J Neurol, 1995. 242(5): p. 304-12.

92. Laloi-Michelin, M., et al., The clinical variability of maternally inherited diabetes and deafness is associated with the degree of heteroplasmy in blood leukocytes. J Clin Endocrinol Metab, 2009. 94(8): p. 3025-30.

93. Suzuki, T. and A. Nagao, Human mitochondrial diseases caused by lack of taurine modification in mitochondrial tRNAs. Wiley Interdiscip Rev RNA, 2011. 2(3): p. 37686.

94. Yasukawa, T., et al., Wobble modification deficiency in mutant tRNAs in patients with mitochondrial diseases. FEBS Lett, 2005. 579(13): p. 2948-52.

95. Kirino, Y., et al., Codon-specific translational defect caused by a wobble modification deficiency in mutant $t R N A$ from a human mitochondrial disease. Proc Natl Acad Sci U S A, 2004. 101(42): p. 15070-5.

96. Chan, S.S. and W.C. Copeland, DNA polymerase gamma and mitochondrial disease: understanding the consequence of POLG mutations. Biochim Biophys Acta, 2009. 1787(5): p. 312-9.

97. Ropp, P.A. and W.C. Copeland, Characterization of a new DNA polymerase from Schizosaccharomyces pombe: a probable homologue of the Saccharomyces cerevisiae DNA polymerase gamma. Gene, 1995. 165(1): p. 103-7.

98. Euro, L., et al., Clustering of Alpers disease mutations and catalytic defects in biochemical variants reveal new features of molecular mechanism of the human mitochondrial replicase, Pol \{gamma\}. Nucleic Acids Res, 2011.

99. Wong, L.J., et al., Molecular and clinical genetics of mitochondrial diseases due to POLG mutations. Hum Mutat, 2008. 29(9): p. E150-E172.

100. Milone, M. and R. Massie, Polymerase gamma 1 mutations: clinical correlations. Neurologist. 16(2): p. 84-91.

101. Yamanaka, H., et al., Novel mutation of human DNA polymerase gamma associated with mitochondrial toxicity induced by anti-HIV treatment. J Infect Dis, 2007. 195(10): p. 1419-25.

102. Pagnamenta, A.T., et al., Dominant inheritance of premature ovarian failure associated with mutant mitochondrial DNA polymerase gamma. Hum Reprod, 2006. 21(10): p. 2467-73.

103. Blok, M.J., et al., The unfolding clinical spectrum of POLG mutations. J Med Genet, 2009. 46(11): p. 776-85.

104. Ashley, N., et al., Depletion of mitochondrial DNA in fibroblast cultures from patients with POLG1 mutations is a consequence of catalytic mutations. Hum Mol Genet, 2008. 17(16): p. 2496-506.

105. Graziewicz, M.A., et al., Structure-function defects of human mitochondrial DNA polymerase in autosomal dominant progressive external ophthalmoplegia. Nat Struct Mol Biol, 2004. 11(8): p. 770-6.

106. Chan, S.S., M.J. Longley, and W.C. Copeland, The common A467T mutation in the human mitochondrial DNA polymerase (POLG) compromises catalytic efficiency and interaction with the accessory subunit. J Biol Chem, 2005. 280(36): p. 31341-6.

107. Del Bo, R., et al., Remarkable infidelity of polymerase gammaA associated with mutations in POLG1 exonuclease domain. Neurology, 2003. 61(7): p. 903-8. 
108. Wanrooij, S., et al., Twinkle and POLG defects enhance age-dependent accumulation of mutations in the control region of mtDNA. Nucleic Acids Res, 2004. 32(10): p. 3053-64.

109. Spelbrink, J.N., et al., In vivo functional analysis of the human mitochondrial DNA polymerase POLG expressed in cultured human cells. J Biol Chem, 2000. 275(32): p. 24818-28.

110. Hance, N., M.I. Ekstrand, and A. Trifunovic, Mitochondrial DNA polymerase gamma is essential for mammalian embryogenesis. Hum Mol Genet, 2005. 14(13): p. 177583.

111. Chan, S.S. and W.C. Copeland, Functional analysis of mutant mitochondrial DNA polymerase proteins involved in human disease. Methods Mol Biol, 2009. 554: p. 5972.

112. de Vries, M.C., et al., Multiple oxidative phosphorylation deficiencies in severe childhood multi-system disorders due to polymerase gamma (POLG1) mutations. Eur J Pediatr, 2007. 166(3): p. 229-34.

113. Chinnery, P., et al., Treatment for mitochondrial disorders. Cochrane Database Syst Rev, 2006(1): p. CD004426.

114. Horvath, R., G. Gorman, and P.F. Chinnery, How can we treat mitochondrial encephalomyopathies? Approaches to therapy. Neurotherapeutics, 2008. 5(4): p. 558-68.

115. Mahoney, D.J., G. Parise, and M.A. Tarnopolsky, Nutritional and exercise-based therapies in the treatment of mitochondrial disease. Curr Opin Clin Nutr Metab Care, 2002. 5(6): p. 619-29.

116. Jeppesen, T.D., et al., Aerobic training is safe and improves exercise capacity in patients with mitochondrial myopathy. Brain, 2006. 129(Pt 12): p. 3402-12.

117. Taivassalo, T., et al., Endurance training and detraining in mitochondrial myopathies due to single large-scale mtDNA deletions. Brain, 2006. 129(Pt 12): p. 3391-401.

118. Jeppesen, T.D., et al., Short- and long-term effects of endurance training in patients with mitochondrial myopathy. Eur J Neurol, 2009. 16(12): p. 1336-9.

119. Clark, K.M., et al., Reversal of a mitochondrial DNA defect in human skeletal muscle. Nat Genet, 1997. 16(3): p. 222-4.

120. Murphy, J.L., et al., Resistance training in patients with single, large-scale deletions of mitochondrial DNA. Brain, 2008. 131(Pt 11): p. 2832-40. 


\section{Chapter 2}

Large scale mtDNA sequencing reveals sequence and functional conservation as major determinants of homoplasmic mtDNA variant distribution.

A.M. Voets, B.J.C. van den Bosch, A.P. Stassen, A.T. Hendrickx, D.M. Hellebrekers, L. Van Laer, E. Van Eyken, G. Van Camp, A. Pyle, S.V. Baudouin, P.F. Chinnery, H.J.M. Smeets

Mitochondrion 2011; 11: 964-972. 


\section{Abstract}

The mitochondrial DNA (mtDNA) is highly variable, containing large numbers of pathogenic mutations and neutral polymorphisms. The spectrum of homoplasmic mtDNA variation was characterized in 730 subjects and compared with known pathogenic sites. The frequency and distribution of variants in protein coding genes were inversely correlated with conservation at the amino acid level. Analysis of tRNA secondary structures indicated a preference of variants for the loops and some acceptor stem positions. This comprehensive overview of mtDNA variants distinguishes between regions and positions which are likely not critical, mainly conserved regions with pathogenic mutations and essential regions containing no mutations at all.

\section{Key words}

MitoChip, mtDNA variants, distribution, pathogenicity 


\section{Introduction}

Mitochondria are essential for the production of ATP, the main source of cellular energy, by the process of oxidative phosphorylation (OXPHOS). Part of the enzyme complexes involved are encoded by the mitochondrial DNA (mtDNA). The human mtDNA consist of 16,569 base pairs and contains 13 OXPHOS protein encoding genes, two ribosomal RNA genes and 22 tRNA genes [1]. It is generally considered that the mtDNA has a higher mutation rate compared to the nuclear DNA (nDNA) which is thought to be due to a combination of less extensive repair mechanisms, the lack of protective histones and closer proximity to a major source of mutation-inducing agents, i.e. the reactive oxygen species (ROS) [2]. The mtDNA is present as multiple copies within the mitochondria of each cell and altered nucleotides can be present in either the homo- or heteroplasmic state [3]. Not long ago it was generally accepted that the most deleterious pathogenic mutations were heteroplasmic, while homoplasmic variants were less severe risk factors or neutral polymorphic variants [4]. However, recent work has revealed a growing list of pathogenic homoplasmic mtDNA variants [5, 6], and most common pathogenic mutations are also present in the general population but usually far below the threshold level for phenotypic expression [7]. This presents a particular challenge when novel genetic variants are detected in patients with mitochondrial disease - conventional criteria cannot reliably determine which variants are phenotypically neutral, and which are actually causing disease. This is especially the case for variants in the protein coding genes and the tRNA genes, the latter compromising more than half of the published pathogenic mtDNA mutations $[4,8]$. Pathogenic rRNA have been described to lesser extent, implicated in for example hearing loss [9]. A number of criteria and tools have been described to facilitate the classification of variants $[10,11]$, but a final conclusion regarding the pathogenicity of a certain variant is not always easy to achieve.

The availability of techniques to generate mutation and/or sequence information of complete mtDNA genomes at a rapid pace has opened new possibilities for the detection and classification of variable and conserved parts of the mtDNA, based on large data sets of mtDNA sequences [12-17]. So far these studies have not been consistent on the frequency and distribution of variants in the mIDNA, both in human and mice. Some report specific hotspots for positions that are altered [11], while others show a more random distribution of variants across the mitochondrial genome [18]. Therefore, we chose to analyze the mtDNA in a large population of 730 European individuals with one technology. We applied the GeneChip ${ }^{\circledR}$ Mitochondria Resequencing array from Affymetrix (MitoChip) as a fast and reliable method to detect homoplasmic variants in the mtDNA [9, 13, 19]. As different approaches to analyze MitoChip data have been described with different sensitivity and specificity for the detection of mtDNA variants [19-21], we had to optimize our procedure. The study cohort contained 218 patients with mitochondrial symptoms, who could also carry pathogenic mutations in the mtDNA, which would create a risk of incidentally including non-neutral variants in the analysis. To minimize this risk we systematically checked all variants identified in this group for pathogenicity. All pathogenic and potentially 
pathogenic mutations, identified in 30 patients, were excluded from analysis and only polymorphisms and, based on the scoring system, predicted benign variants were included. This, together with the size of the entire cohort in majority of individuals with no mitochondrial disorders, prevents that the analysis is influenced by inclusion of pathogenic mutations. In our paper, we present novel data regarding the distribution of homoplasmic variants across the mtDNA and we provide clues to help distinguish neutral (homoplasmic) variants from highly deleterious pathogenic mutations in the tRNA genes.

\section{Material and methods}

\section{DNA extraction}

Total cellular DNA was isolated from muscle or blood from 730 subjects (supplementary table 2.1) according to standard procedures. Of these, 400 subjects were inhabitants with age-related hearing impairment from a residential village of Antwerp (Belgium), between 54 and 66 years old, invited through population registries [22]. A total of 218 subjects were patients with a variety of mitochondrial symptoms and ages between 0 and 50 years, referred to the Clinical Genetics department in Maastricht (the Netherlands). For those subjects, the pathogenic or likely pathogenic mutation, when found, was added to supplementary table 2.1 and was not included in the further analysis. The remaining 112 subjects (with age from 54 to 75 ) were sequential admissions to the Critical Care Unit in Newcastle upon Tyne (United Kingdom). This cohort had an identical haplogroup distribution to the background population from the same geographical region, and thus is considered to be a random sample of individuals living in the North East of England [23]. All known or likely pathogenic mutations (supplementary table 2.2) in the entire cohort were excluded from the analysis.

\section{MitoChip and experimental procedure}

GeneChip ${ }^{\circledR}$ Mitochondria Resequencing 2.0 Arrays (Affymetrix, Santa Clara, CA, USA) were used to determine the sequence of the 730 DNA samples. In short, the entire mtDNA was amplified using the Expand Long Template system (Roche, Almere, the Netherlands) in 2 fragments (A and B) of 8207 bp and 8545 bp in length, with an overlap of $183 \mathrm{bp}$. The primers for fragment A were forward primer [5'cgttccagtgagttcaccct-3'] and reverse primer [5'-ggtaagaagtgggctagggc-3'], and for fragment B forward primer [5'-taaacctagccatggccatc-3'] and reverse primer [5'tgtggctaggctaagcgttt-3']. PCR products were purified using the QIAQuick PCR cleanup kit (Qiagen). Equimolar amounts of the amplified fragments $A$ and $B$ were pooled and fragmented, labeled and hybridized on a pre-hybridized GeneChip according to the GeneChip CustomSeq Resequencing Array Affymetrix protocol. Chips were washed and stained on the GeneChip fluidics station 450 (Affymetrix) using the preprogrammed wash and stain protocol (Mini_DNAARRAY_WS5_450). The chips were scanned using the Affymetrix GeneChip scanner 3000 creating CEL files for subsequent analysis. 


\section{Data analysis}

After generation of the cell intensity (CEL) files by GeneChip ${ }^{\circledR}$ Operating Software 1.4 (GCOS 1.4), raw sequence data was obtained by the GeneChip ${ }^{\circledR}$ Sequence Analysis Software 4.1 (GSEQ 4.1, Affymetrix) and Sequence Pilot - module SeqC (JSI medical systems) according to the flow chart depicted in figure 2.1. GSEQ uses an objective statistical framework, based upon the ABACUS algorithm [24] to assign base calls to each position which meets quality criteria in the mitochondrial genome, based on consistent calls of both strands. GSEQ analysis was performed using the haploid model with quality score threshold (QST) 3. SeqC automatically uses all previously made analyses as controls for the actual samples and therefore is able to anticipate the shape of every peak in the software output. Dissimilarity scores are calculated for every peak and are a measure for the deviation of the peak areas from the statistical average. A warning is displayed for any peak with an abnormal high dissimilarity score. The SeqC software was used with the standard parameters. The eventual mtDNA sequences were compared with the revised Cambridge reference sequence to list all homoplasmic variants.

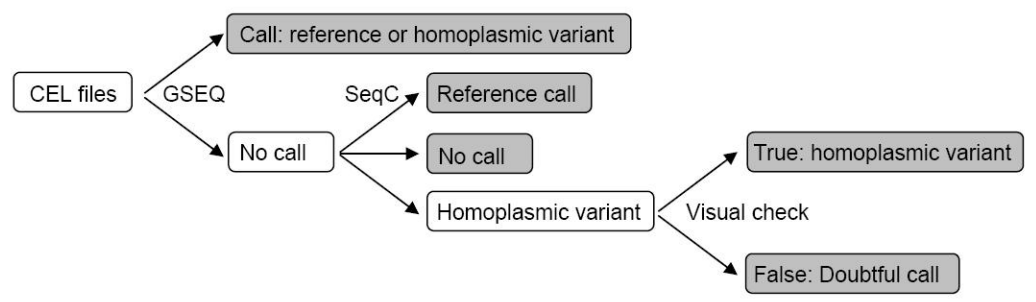

Figure 2.1. Data analysis flow chart.

The haplogroups were determined according to the presence or absence of the haplogroup variants listed in supplementary table 2.3. The best fitting haplogroup was assigned. The sequences of the seven species used for examining conservation of the mitochondrial genes were derived from NCBI: H. sapiens NC_012920; C. lupus NC_002008.4; B. taurus NC_006853.1; M. musculus NC_005089.1; R. norvegicus NC_001665.2; G. gallus NC_001323.1; D. melanogaster NC_001709.1. A filtered list of pathogenic tRNA and protein mtDNA mutations was created from a list of disease associated mtDNA mutations in the MITOMAP database (www.mitomap.org/MITOMAP, supplementary table 2.2). Variants of which the pathogenicity status was listed in Mitomap as 'unclear', 'polymorphism', 'synergistic', 'conflicting reports', 'secondary', 'haplogroup marker' or 'warrants further study', or was 'unconvincing' in the corresponding publication were not included. All inherited pathogenic mutations (Mitomap 'confirmed') were included. For the remaining variants (Mitomap 'reported'), a pathogenicity score was calculated for the tRNA and protein coding mutations according to a checklist. For protein coding genes, the scoring system described previously [25] was used. Additionally, the conservation of the variant position (mtSNP http://mtsnp.tmig.or.jp/mtsnp/index_e.shtml; Alamut, Interactive Biosoftware) and the effect of the variant on polarity, protein structure/function (PolyPhen http://genetics.bwh.harvard.edu/pph/; SIFT $\quad$ http://sift.jcvi.org/www/ 
SIFT_BLink_submit.html; InterProScan http://www.ebi.ac.uk/Tools/InterProScan/; UniProt http://www.uniprot.org/ uniprot/; TMHMM http://www.cbs.dtu.dk /services/TMHMM/; MSOP http://npsa-pbil.ibcp.fr/cgi-bin/npsa_automat.pl?page= npsa_sopm.html) were evaluated. For the tRNA genes, another scoring system was used [11]. Additionally, the conservation of nucleotide and the effect of the variant on secondary or tertiary interactions (Mamit-tRNA http://mamit-trna.u-strasbg.fr/) within the tRNA molecule were evaluated. For all variants, the presence of the variant in general databases (mtSNP, mtDB http://www.genpat.uu.se/mtDB/, OMIM, PubMed, Google) was checked. Variants that were scored definitely or highly likely pathogenic were included in the list of pathogenic mutations and excluded from further analysis. Correlation between the number of variants and the variant intensity of guanine $(G)$ residues in the protein coding genes was analyzed using the linear regression function of SPSS.

\section{Results}

Analysis of the entire mtDNA sequence in 730 subjects

The mtDNA of 730 subjects was sequenced using the MitoChip and sequences were compared with the revised Cambridge Reference Sequence (rCRS). Because of the lower sensitivity and specificity of the MitoChip for the detection of heteroplasmic variants [19], the analysis was restricted to homoplasmic variants. After analysis with GSEQ, $1.3 \%$ (standard error 0.03 ) of the 16544 nucleotides in the 730 samples gave a no call. The main reason was a low signal, a repetitive sequence or a C-stretch in one of the two strands (data not shown). These regions were predominantly located in the D-loop of the mtDNA and less in the protein and RNA coding genes. The D-loop was excluded from the analysis. The distribution of no calls was not homogeneous and some genes (e.g. ATP8) showed a significantly higher percentage of nucleotides that were not called than others (e.g. ND4L) (table 2.1). Therefore, to reduce the number of bases with a no call, Sequence Pilot - module SeqC was used for the positions that were not called by GSEQ. Additional variants called by SeqC were checked visually. Variants scored by SeqC were only called confirmed when both forward and reverse strand clearly showed the mutant and no wild type peak. When a wild type peak was visible on one of the strands, the variant call from SeqC was depicted as 'doubtful'. When both GSEQ and SeqC could not call a position, it remained a 'no call'. SeqC analysis decreased the percentage of no calls from 1.096 to 0.001 (excluding the Dloop) without adding many extra different homoplasmic variants or doubtful calls. The distribution of the no calls over the different genes was more homogeneous (table 2.1). Five randomly chosen additional SeqC variants were conventionally sequenced and confirmed (data not shown). 
Table 2.1. Results of variant and 'no call' analysis using the GSEQ and SeqC analysis pipeline.

\begin{tabular}{|c|c|c|c|c|c|c|c|}
\hline \multirow[b]{2}{*}{ Gene } & \multicolumn{3}{|c|}{ GSEQ analysis } & \multicolumn{4}{|c|}{ SeqC analysis ${ }^{a}$} \\
\hline & $\begin{array}{c}\text { \# different } \\
\text { variants }\end{array}$ & $\begin{array}{l}\text { \# no } \\
\text { calls }\end{array}$ & $\begin{array}{c}\% \text { ntds with } \\
\text { no call }\end{array}$ & $\begin{array}{c}\text { \# different } \\
\text { variants }\end{array}$ & $\begin{array}{l}\text { \# no } \\
\text { calls }\end{array}$ & $\begin{array}{l}\% \text { ntds with } \\
\text { no call }\end{array}$ & $\begin{array}{c}\text { \# doubtful } \\
\text { calls }\end{array}$ \\
\hline ATP6 & $89^{*}$ & 16809 & 3.381 & $91^{*}$ & 68 & 0.014 & 8 \\
\hline ATP8 & $26^{*}$ & 9276 & 6.139 & $27^{\star}$ & 3 & 0.002 & 2 \\
\hline$C Y B$ & 115 & 10615 & 1.274 & 115 & 9 & 0.001 & 6 \\
\hline $\mathrm{COI}$ & 129 & 8245 & 0.732 & 130 & 4 & $<0.001$ & 3 \\
\hline COII & 53 & 9473 & 1.897 & 57 & 11 & 0.002 & 2 \\
\hline COIII & 69 & 3442 & 0.601 & 71 & 0 & 0.000 & 9 \\
\hline$N D 1$ & 95 & 9344 & 1.338 & 96 & 5 & $<0.001$ & 16 \\
\hline$N D 2$ & 113 & 10518 & 1.383 & 114 & 16 & 0.002 & 53 \\
\hline$N D 3$ & 45 & 1383 & 0.548 & 45 & 2 & 0.001 & 10 \\
\hline$N D 4$ & 112 & 9126 & 0.907 & 114 & 15 & 0.001 & 10 \\
\hline$N D 4 L$ & 29 & 228 & 0.105 & 29 & 1 & $<0.001$ & 2 \\
\hline ND5 & 185 & 15900 & 1.202 & 187 & 3 & $<0.001$ & 12 \\
\hline$N D 6$ & 51 & 6562 & 1.712 & 55 & 1 & $<0.001$ & 174 \\
\hline TRNF & 2 & 329 & 0.635 & 2 & 2 & 0.004 & 0 \\
\hline TRNV & 1 & 4 & 0.008 & 1 & 0 & 0.000 & 1 \\
\hline TRNL1 & 1 & 1 & 0.002 & 1 & 0 & 0.000 & 0 \\
\hline TRNI & 4 & 322 & 0.639 & 4 & 2 & 0.004 & 0 \\
\hline TRNQ & 3 & 58 & 0.110 & 4 & 0 & 0.000 & 3 \\
\hline TRNM & 3 & 104 & 0.210 & 3 & 0 & 0.000 & 0 \\
\hline TRNW & 4 & 19 & 0.038 & 4 & 0 & 0.000 & 0 \\
\hline TRNA & 4 & 29 & 0.058 & 4 & 0 & 0.000 & 1 \\
\hline TRNN & 0 & 1 & 0.002 & 0 & 0 & 0.000 & 0 \\
\hline TRNC & 9 & 3 & 0.006 & 9 & 0 & 0.000 & 0 \\
\hline$T R N Y$ & 2 & 8 & 0.017 & 2 & 0 & 0.000 & 0 \\
\hline TRNS1 & 2 & 353 & 0.711 & 2 & 1 & 0.002 & 23 \\
\hline TRND & 3 & 244 & 0.492 & 3 & 0 & 0.000 & 4 \\
\hline TRNK & 2 & 35 & 0.068 & 2 & 0 & 0.000 & 1 \\
\hline TRNG & 4 & 26 & 0.052 & 4 & 0 & 0.000 & 0 \\
\hline TRNR & 3 & 579 & 1.220 & 3 & 1 & 0.002 & 20 \\
\hline TRNH & 5 & 23 & 0.046 & 5 & 2 & 0.004 & 0 \\
\hline TRNS2 & 2 & 255 & 0.592 & 2 & 0 & 0.000 & 0 \\
\hline TRNL2 & 2 & 163 & 0.314 & 2 & 0 & 0.000 & 2 \\
\hline TRNE & 5 & 0 & 0.000 & 5 & 0 & 0.000 & 0 \\
\hline TRNT & 16 & 96 & 0.199 & 16 & 0 & 0.000 & 0 \\
\hline TRNP & 0 & 75 & 0.151 & 0 & 0 & 0.000 & 0 \\
\hline$R N R 1$ & 38 & 3340 & 0.480 & 39 & 0 & 0.000 & 0 \\
\hline RNR2 & 79 & 3852 & 0.338 & 80 & 5 & $<0.001$ & 5 \\
\hline $\begin{array}{l}\text { Total } \\
\text { analyzed }^{c}\end{array}$ & 1317 & 123582 & 1.096 & 1341 & 150 & 0.001 & 394 \\
\hline
\end{tabular}




\section{Table 2.1 legend}

${ }^{\text {a}}$ Positions which were not called by GSEQ analysis were analyzed with the SeqC software and newly identified variants were checked visually. When the SeqC call was wrong, the position was recorded as a 'doubtful call'.

${ }^{\mathrm{b}}$ Average percentage of nucleotides that were not called for 730 subjects (total number of no calls/[length of the sequence $\times 730$ subjects] $\times 100$ )

${ }^{\mathrm{c}}$ The mtDNA sequence was analyzed from position 577 to position 16023.

* ATP6 and ATP8 have 8 variants in common

Characterization of variants in the mtDNA

General features of the variants identified

A total of 12,063 homoplasmic variants (average 16.5 variants per subject) were detected relative to the rCRS, of which 1,341 (average 1.8 per subject) were different variants at 1,321 (average 1.8 per subject) different positions in the mtDNA (supplementary table 2.4). The large amount of total variants is due to the presence of European haplogroup variants which were present in a high proportion of the subjects (haplogroup and number of subjects: H 307, U 99, K 59, HV 52, J 64 and JT 73; supplementary table 2.1). The haplogroup distribution was comparable among the three cohorts (supplementary table 2.5), pointing to a generally European population, and therefore the three cohorts were merged for further analysis. Furthermore, to minimize confounding by the haplogroup variants, variant numbers mentioned in the analyses are based on the 1,341 different variants.

Table 2.2. Overview of observed variants by nucleotide.

\begin{tabular}{|c|c|c|c|c|c|c|c|}
\hline \multicolumn{6}{|c|}{ Observed number of variants ${ }^{a}$} & \multirow{2}{*}{$\begin{array}{l}\text { Number of } \\
\text { nucleotide }^{b}\end{array}$} & \multirow{2}{*}{$\begin{array}{c}\text { Variant } \\
\text { Intensity }^{c}\end{array}$} \\
\hline reflvar & $\mathrm{A}$ & $\mathrm{G}$ & C & $\mathrm{T}$ & Total & & \\
\hline \multirow[t]{2}{*}{ A } & - & 383 & 23 & 19 & 425 & 4785 & 1.03 \\
\hline & & & & & $(32 \%)$ & $(31 \%)$ & \\
\hline \multirow[t]{2}{*}{ G } & 228 & - & 7 & 2 & 237 & 2017 & 1.35 \\
\hline & & & & & $(18 \%)$ & $(13 \%)$ & \\
\hline \multirow[t]{2}{*}{ C } & 31 & 13 & - & 239 & 283 & 4810 & 0.68 \\
\hline & & & & & $(21 \%)$ & $(31 \%)$ & \\
\hline \multirow[t]{2}{*}{$\mathrm{T}$} & 13 & 16 & 367 & - & 396 & 3833 & 1.19 \\
\hline & & & & & $(30 \%)$ & $(25 \%)$ & \\
\hline Total & 272 & 412 & 397 & 260 & 1341 & 15446 & \\
\hline
\end{tabular}

${ }^{a}$ Observed number of different variants according to the revised Cambridge Reference Sequence (rCRS)

${ }^{b}$ The number of nucleotide $x$ in the mtDNA excluding the displacement loop (position 577-16023)

${ }^{c}$ Variant intensity is calculated as follows: (observed number of variants nucleotide $x /$ total number of nucleotide $\mathrm{x}$ )/(total number of variants/total number of nucleotides)

The observed frequency of variants for each of the different nucleotides (A, C, G, T) according to the rCRS are shown in table 2.2. Variants changing nucleotide $A$ were most frequent. To correct for differences in the total number of the four nucleotides in the mtDNA regions tested, the variant intensity [26] was calculated. This variant intensity corrected for the presence of the nucleotide in the mtDNA (excluding the D- 
loop) by comparing this value with the prevalence of all variants in the entire mtDNA (excluding the D-loop) (formula: [number of variants nucleotide $\mathrm{x} /$ total number of nucleotide $\mathrm{x}]$ [/total number of variants/total number of nucleotides]). The variant intensity is depicted in table 2.2 and is increasing in the order $\mathrm{C}<\mathrm{A}<\mathrm{T}<\mathrm{G}$. Although nucleotide $G$ showed the smallest number of variants, it had the highest variant intensity.

\section{Protein coding variants}

The number of variants in the different protein coding genes was assessed (figure 2.2A). After correction for the length of the gene, differences in the prevalence of variants could be observed. On average, $10.4 \%$ of the nucleotides in the protein coding genes showed a variant in one of the 730 subjects $(0.104$ variants/base pair) While ATP6, ATP8 and ND3 showed the highest number of variants, COI, COII and ND4 contained fewer variants per base pair. These differences were mainly due to differences in variant numbers on codon position 1 and 2 (figure 2.2A). In general, it was observed that the number of variants at codon position 1 ( 0.070 variants/base pair) and 2 (0.036 variants/ base pair) was lower compared with the $3^{\text {rd }}$ position $(0.192$ variants/base pair) (figure 2.3). Codon position 3 variants predominantly consisted of synonymous amino acid changes, whereas codon position 1 showed only a small proportion of synonymous variants and codon position 2 variants consisted entirely of non-synonymous variants.

The frequency of Cs (lowest variant intensity) or Gs (highest variant intensity) in the sequence of the genes (all positions or only third codon positions) could not explain the discrepancy in the number of variants between the different genes (data not shown). However, there was a significant $(p=0.004)$ correlation between the number of variants and the variant intensity of $\mathrm{G}$ in the protein coding genes (figure 2.2B). ND6 was not included in the analysis as this gene is transcribed from the L-strand and the $G$ content differs from the $\mathrm{H}$-strand. To check for a role of evolutionary conservation in the distribution of the variants, COI (low number of variants/bp) and ATP6 (high number of variants/bp) were examined in more detail in seven species (H. sapiens, G. gallus, C. lupus, B. taurus, M. musculus, R. norvegicus and D. melanogaster). Whereas more than half of the nucleotides in the $\mathrm{CO}$ gene were conserved in seven species, approximately half of the variants were located at positions conserved in less than four species (figure 2.4A). In contrast, the ATP6 sequence was less well conserved (figure 2.4A) but the preference of the variants for the less conserved positions in ATP6 was lower compared with $\mathrm{COI}$ variants (figure 2.4A). However, no such difference could be observed when only the third codon positions were analyzed (figure 2.4B). The same trend in sequence conservation was shown for COII and COIII (low number of variants/bp) and ATP8 and ND3 (high number of variant/bp) but the preference of variants for less conserved first and second positions between the genes was less discriminating (supplementary figure 2.1). The 67 pathogenic mutations that passed our filter were all, except for five deletions, located on first and second codon positions and were mainly targeting highly conserved positions (57/67 conserved in at least 6 species; supplementary table 2.2). 
A

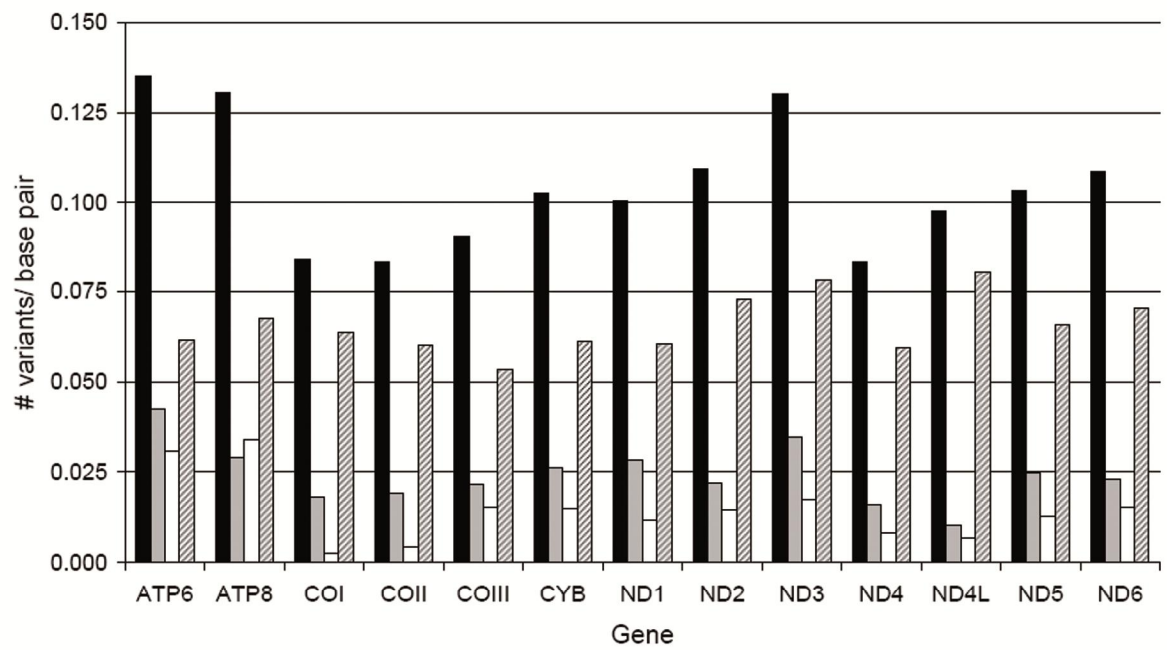

- Total Gene $\square$ Codon 1 Codon 2 v Codon 3

B

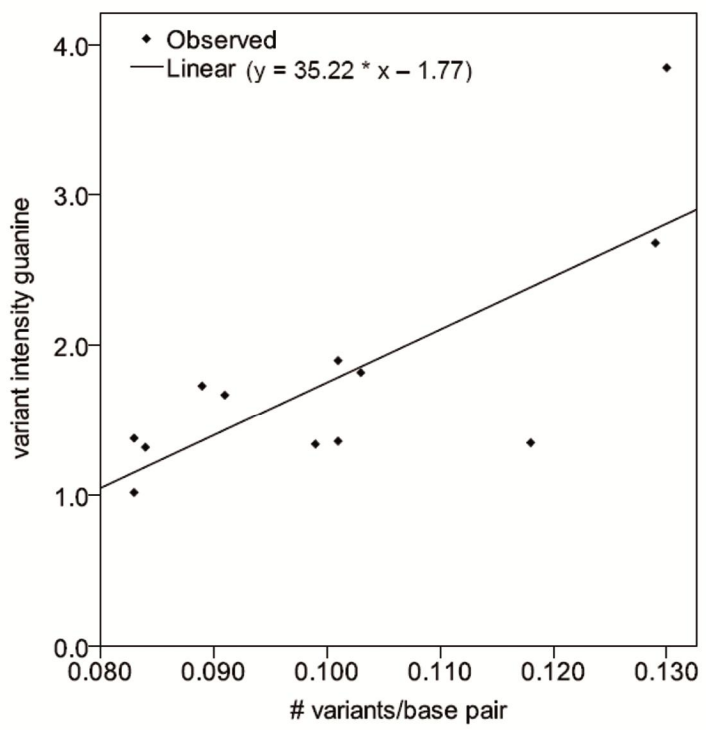

Figure 2.2. Variant distribution by protein coding gene. A The observed number of variants in the whole cohort is expressed as the number of variants per base pair to correct for the length of the genes and is shown for the total gene as well as for the three different codon positions. B Significant $(p=$ 0.004 ) correlation between the number of variants and the variant intensity of guanine in the protein coding genes (excluding ND6). 


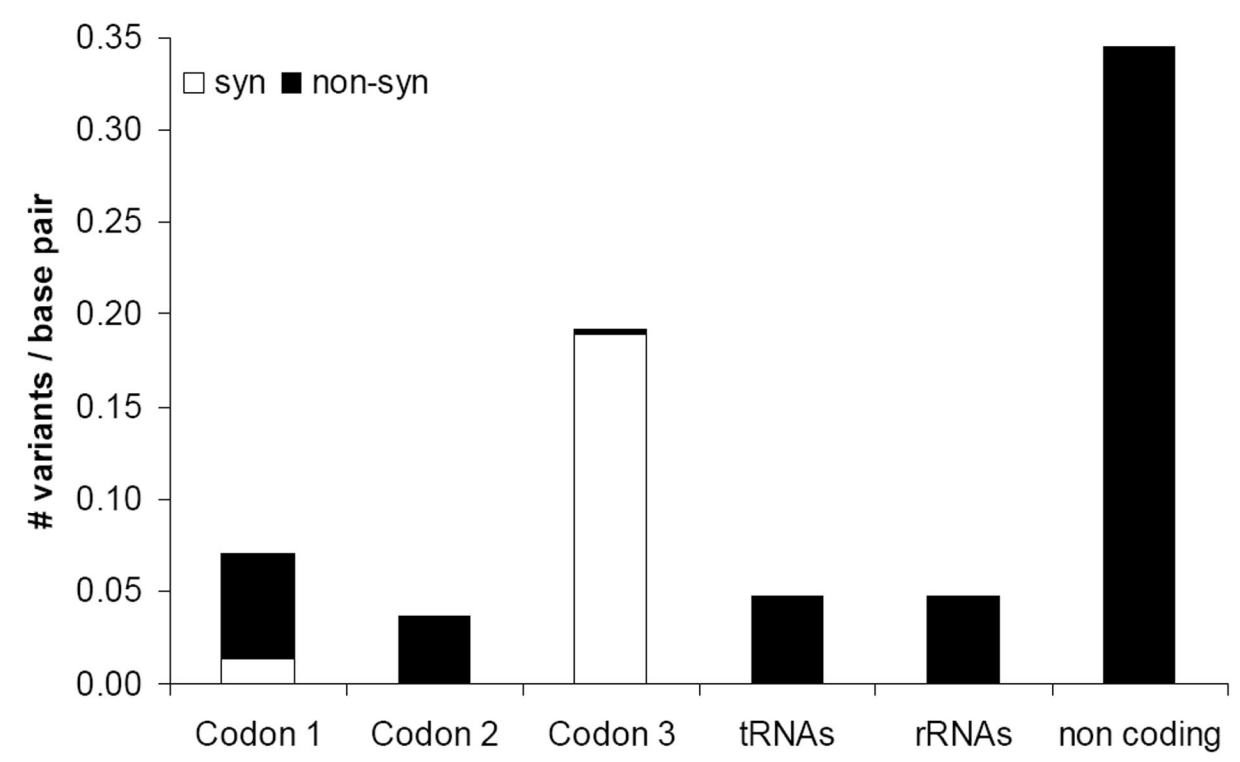

Figure 2.3. Variant distribution in protein coding genes by codon position, tRNA and rRNA genes and non-coding nucleotides. The observed number of variants in the whole cohort is expressed as the number of variants per base pair of each sequence type. The proportion of synonymous and nonsynonymous variants is indicated.

\section{$\underline{\text { tRNA variants }}$}

In the 730 samples, only 78 different homoplasmic tRNA variants were detected. On average, $5.3 \%$ of the positions showed a variant in one of the 730 subjects $(0.053$ variants/base pair), in between the rate observed for codon positions 1 and 2 of the protein coding genes (figure 2.3). The location and positional conservation of these variants were compared with a list of known pathogenic tRNA mutations (supplementary table 2.2) to detect discriminating characteristics (table 2.3). For the homoplasmic variants, approximately half $(52 \%)$ of the variants were located in the loops of the tRNA whereas only $32 \%$ of the pathogenic mutations were located here. Homoplasmic variants were representing, among others, poorly conserved mismatch to Watson-Crick base pair (WC) changes and both poorly and highly conserved mismatch to mismatch changes in the tRNA stems, which were not present among the pathogenic mutations. While three pathogenic mutations changed WCs into severe mismatches (not A.C, C.A, G.U or U.G) on conserved positions, no homoplasmic variants caused such changes. Furthermore, when examining the conservation of the base pairing (conserved WC, G.U or mismatch base pairs), pathogenic mutations predominated in conserved base pairs compared with the homoplasmic variants (34/90 pathogenic mutations versus $7 / 78$ homoplasmic variants). 

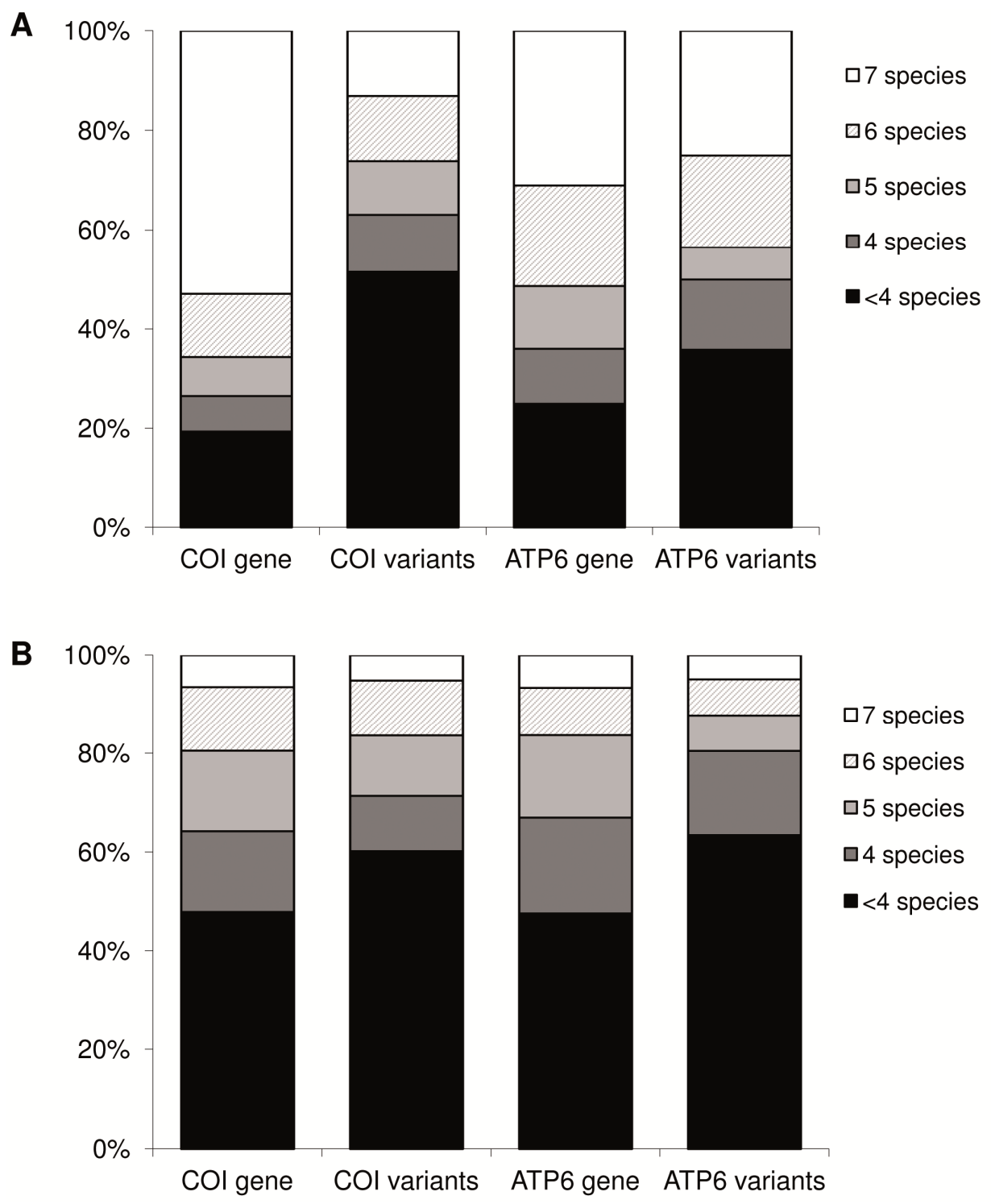

Figure 2.4. Conservation of total gene sequence and variant positions in COI and ATP6 for all codon positions (A) and third codon positions (B). The human sequence of both genes was aligned with the sequence of six other species: G. gallus, C. lupus, B. taurus, M. musculus, R. norvegicus and D. melanogaster. 
Table 2.3. Location and conservation characteristics of homoplasmic tRNA variants and pathogenic tRNA mutations.

\begin{tabular}{|c|c|c|c|c|c|c|}
\hline \multirow[b]{2}{*}{ Location } & \multirow[b]{2}{*}{ Change $^{a}$} & \multirow[b]{2}{*}{$\begin{array}{c}\text { Positional } \\
\text { conservation }^{\mathrm{b}}\end{array}$} & \multicolumn{2}{|c|}{ homoplasmic variants } & \multicolumn{2}{|c|}{ pathogenic mutations $^{c}$} \\
\hline & & & $\begin{array}{c}\text { total \# } \\
(\%)\end{array}$ & $\begin{array}{c}\text { \# conserved } \\
\text { pairing }^{d}\end{array}$ & $\begin{array}{l}\text { total \# } \\
(\%)\end{array}$ & $\begin{array}{c}\text { \# conserved } \\
\text { pairing }^{d}\end{array}$ \\
\hline \multirow[t]{2}{*}{ loop } & & $<100 \%$ & $37(47)$ & & $11(12)$ & \\
\hline & & $100 \%$ & $4(5)$ & & $18(20)$ & \\
\hline \multirow[t]{8}{*}{ stem } & $M M>W C$ & $<100 \%$ & $\underline{13(17)}$ & 2 & $0(0)$ & \\
\hline & $\mathrm{MM}>\mathrm{WC}$ & $100 \%$ & $1(1)$ & 1 & $2(2)$ & 1 \\
\hline & $\mathrm{MM}>\mathrm{MM}$ & $<100 \%$ & $\underline{3(4)}$ & & $0(0)$ & \\
\hline & $\mathrm{MM}>\mathrm{MM}$ & $100 \%$ & $\underline{1(1)}$ & & $0(0)$ & \\
\hline & $W C>m M M$ & $<100 \%$ & $16(21)$ & 1 & $31(34)$ & 13 \\
\hline & $W C>m M M$ & $100 \%$ & $3(4)$ & 3 & $25(28)$ & 20 \\
\hline & WC>sMM & $<100 \%$ & $0(0)$ & & $0(0)$ & \\
\hline & WC>sMM & $100 \%$ & $0(0)$ & & $\underline{3(3)}$ & \\
\hline Total & & & 78 & 7 & 90 & 34 \\
\hline
\end{tabular}

Underlined data represent types of variants only present in the homoplasmic variants or pathogenic mutations group

${ }^{a} \mathrm{MM}>\mathrm{WC}=$ mismatch base pair to Watson-Crick base pair (WC)

$\mathrm{MM}>\mathrm{MM}=$ mismatch base pair to another mismatch base pair

$W C>m M M=W C$ to a mild mismatch base pair, mild mismatch base pairs are $A \cdot C, C \cdot A, G \cdot U$ and $U \cdot G$

$W C>S M M=W C$ to a severe mismatch base pair, severe mismatch base pairs are all mismatch base pairs excluding $A \cdot C, C \cdot A, G \cdot U$ and $U \cdot G$

${ }^{b}$ as determined in 31 mammalian mitochondrial genomes [27]

${ }^{\mathrm{c}}$ see supplementary table 2.3 for detailed list

${ }^{d}$ conserved pairing: $100 \%$ conserved WC or $>90 \% \mathrm{G} \cdot \mathrm{U}$ and $\mathrm{U} \cdot \mathrm{G}$ as determined in 31 mammalian mitochondrial genomes [27]

Finally, a heat map of all 22 tRNA secondary structures was generated according to the tRNA numbering to examine positional differences between homoplasmic variants and pathogenic mutations in more detail. In this way, the homoplasmic variants and pathogenic mutations in all tRNA molecules could be visualized in one figure (figure 2.5). Homoplasmic variants were located more often in the tRNA loops (except for the anticodon) and some positions of the acceptor stem compared with the pathogenic mutations (figure $2.5 \mathrm{~A}$ and $\mathrm{B}$ ). Again, it is clear that pathogenic mutations in the stem almost always cause WC to mismatch changes while a significant proportion of homoplasmic variants represents mismatch to mismatch or mismatch to WC changes (figure $2.5 \mathrm{~A}$ and $\mathrm{B}$ ). It can be observed that pathogenic mutations are dominating the anticodon, the anticodon stem, the $D$ stem and part of the acceptor stem of the tRNAs (figure 2.5C).F 
Figure 2.5
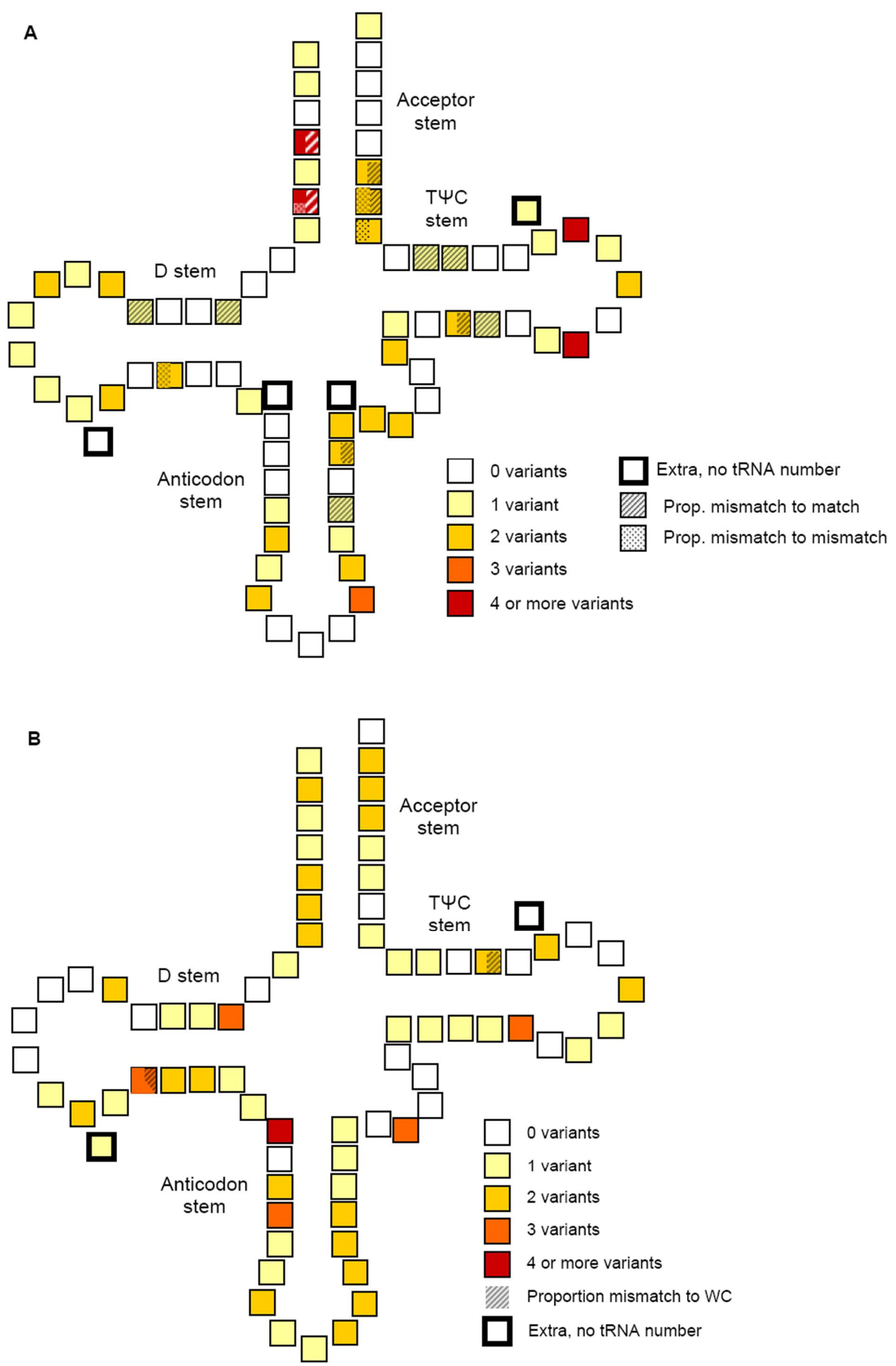


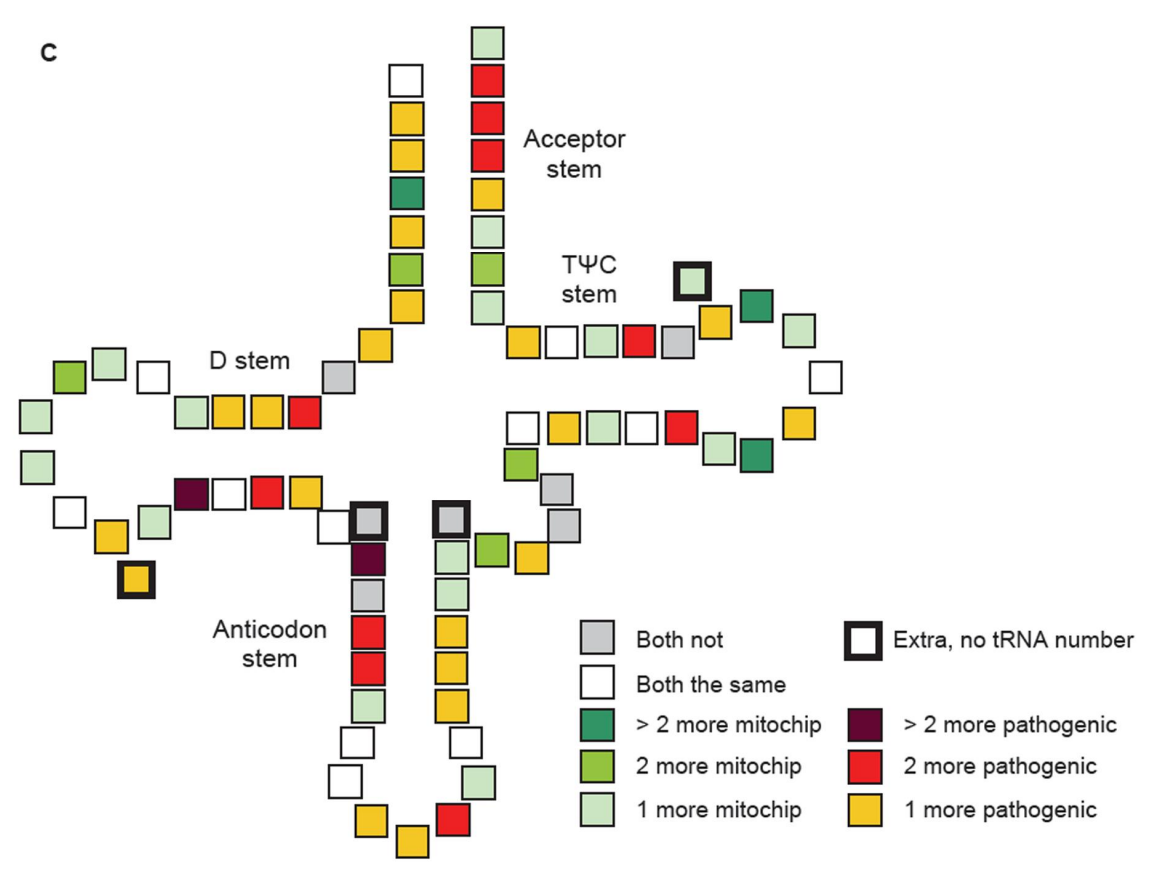

Figure 2.5. Composite of all 22 tRNA secondary structures with variant/mutation locations. All tRNAs are merged into one figure according to tRNA number. Frequencies of homoplasmic variants (A) and pathogenic mutations (B) are appointed to each tRNA position with different colors. Stripes and dots represent the proportion of mismatch to WC or mismatch to mismatch changes, respectively, comparable to the area that is indicated. No indication means WC to mismatch change. $\mathbf{C}$ depicts the difference in number of homoplasmic variants and pathogenic mutations for each tRNA position.

\section{rRNA variants and non-coding regions}

The $12 S$ and $16 S$ rRNA genes showed variants with a density of 0.041 and 0.051 different variants/base pair, respectively. These numbers are only half of the average protein coding genes but are in the same range as the average variant density in tRNA genes and codon position 1 and 2 of the protein coding genes (figure 2.3), indicating a comparable selective pressure. Non-coding nucleotides $(n=58)$ between protein coding, rRNA and tRNA genes showed the highest number of variants with an average of 0.345 different variants per position (figure 2.3). 


\section{Discussion}

In this study, the mtDNA of 730 subjects was analyzed using the Affymetrix MitoChip v2.0. Sequence data analysis was improved to increase the call rate without increasing the number of false positives or false negatives significantly. Homoplasmic mtDNA variants were scored to explore variable and evolutionary conserved regions.

\section{Pipeline for identifying and analyzing mtDNA mutations}

Compared with conventional sequencing, MitoChip resequencing is a fast and cost effective method to screen the whole mtDNA for homoplasmic and heteroplasmic mutations [13]. The mtDNA of 730 subjects was resequenced using the Affymetrix MitoChip v2.0 and base calling was performed by the GSEQ software. It has been shown previously [19] that the MitoChip has lower sensitivity and specificity in the detection of heteroplasmic variants. In our diagnostic experience (data not shown) the call rate of the MitoChip is $>98 \%$ and the sensitivity to detect heteroplasmic mutation ranges from 5 to $30 \%$, which may differ for different nucleotide positions. This implies that the in muscle DNA most relevant causal heteroplasmic mutations will be detectable. Massive parallel sequencing technology will provide an alternative, especially when prices will drop and the required coverage can be reached in a costeffective way. By massively parallel sequencing-by-synthesis, it was observed that in nearly all of the normal tissues, somatic low level heteroplasmic variants $(>1.6 \%$ heteroplasmy) were present [28]. However, the relevance of detecting such low heteroplasmy levels in relation to phenotypic expression is debatable. The MitoChip has been used in studies concerning the influence of homoplasmic polymorphisms on disease (progression) [29, 30] and anthropological studies [19]. We also concentrated on homoplasmic variants only [4]. Highly deleterious pathogenic mutations will very rarely reach homoplasmy because homoplasmy is likely to be lethal at the cellular level [18]. This is in contrast to neutral or adaptive heteroplasmic variants, which become homoplasmic or are lost within 70 cell divisions [31].

GSEQ has been reported to generate very few false negatives and false positives but a high number of no calls [19, 32], as was also observed in this study. The alternative local context probes on the MitoChip v2.0 did not provide a solution for this problem, since these probes were mainly located in the D-loop. The use of additional algorithms or masks following GSEQ analysis has been shown to improve the average call rate [19-21, 32]. In our study, the combination of the haploid model of GSEQ followed by analysis using SeqC (with a higher call rate [33]) turned out to be a sensitive and efficient approach. A key difference between the software programs with respect to the number of no-calls is the ability of SeqC to call a position based on one strand, where as GSEQ requires consistent calls on both strands. Furthermore, SeqC uses all previously analyzed samples as controls for the actual samples which enables a statistically validated continuous software learning process. To control for false positives, we used both software tools and all additional variants called by SeqC were checked visually. Conclusively, the current protocol was feasible for the number of 
MitoChips studied, making this method applicable for the analysis of MitoChip data in a large cohort of subjects.

\section{Characterization of mtDNA variants}

\section{General features}

In 730 subjects, 12,063 homoplasmic variants were detected in the mtDNA excluding the D-loop. D-loop variations (especially the hypervariable segments) have been characterized extensively to study variation within and between human populations [3437] and were not considered further in this study. The variant intensity analysis indicated that nucleotides $\mathrm{G}$ and $\mathrm{C}$ proportionally presented with the highest and lowest variant intensities compared with $A$ and $T$, respectively. This is in agreement with previously published results that showed guanine to be the least stable nucleotide and cytosine the most stable nucleotide while adenine and thymine had intermediate stability [26, 38]. Differences in variant intensity however can only occur when there are strand-specific biases in substitution rates. This theory is reinforced by the deviation from the theoretical $A=T$ and $G=C$ nucleotide composition within each strand when no strand-specific bias would exist [39]. Multiple other studies have shown strand asymmetry in mutation rates [40]; this is probably due to periods of separation of the two DNA strands during replication, which may lead to increased mutability, and transcription-coupled repair [41, 42].

\section{Protein coding variants}

Our data confirmed not unexpectedly the strong negative selection against variants on codon position 1 and 2 due to the non-synonymous nature of variants at these positions $[43,44]$. Not all protein coding genes accumulated the same number of variants which was correlated with a heterogeneous guanine mutation rate. Previously, it was suggested that different mutation rates of mitochondrial genes are at least partly independent of protein structure and function and a property of the mtDNA itself [26]. The authors based their theory on segmentation of the mtDNA by differences in nucleotide composition and that the segments showed a heterogeneous guanine mutation rate. Our study indicated that the heterogeneous guanine mutation rate not only applies to the different mtDNA segments described previously [26], but also plays a role in the individual genes. The protein coding gene results largely correspond with a previous study using data from the mtDNA mutator mice and the human mtDB database [43].

The differences in variant accumulation in the protein coding genes may also be due to differences in selective pressure at the functional (amino acid) level. For the highly conserved $\mathrm{COI}$ gene, variants were mainly located on less conserved positions whereas the less well conserved ATP6 gene showed weaker preference for variants on these positions. If only third codon positions were considered in the analysis, then no differences were observed between the genes. Conclusively, the hypothesis of stronger selection against variants in genes with a high level of sequence conservation and more tolerance for variants in less well conserved genes [43], seems valid in this data set but it only applies for functionally relevant first and second codon positions. In 
agreement with this, differences in variant numbers between the different genes are reflected by differences in variant numbers on codon positions 1 and 2 , whereas variant numbers on positions with minor effect on protein function (codon position 3) are more or less comparable among all genes. The role of functional preservation and conservation is further emphasized by the location of the filtered list of pathogenic mutations (supplementary table 2.2) of which most were on highly conserved functionally relevant positions.

\section{tRNA, rRNA and non-coding region variants (except D-loop)}

The position and effect of the 78 different tRNA variants in the present data set were compared with the position and effect of the known pathogenic mutations (supplementary table 2.2). As mentioned previously [11, 45], for many mtDNA variants listed as pathogenic mutations in the MITOMAP database, insufficient evidence is presented to confidently ascribe pathogenicity to these variants. For example, 40 of the 171 different mutations listed as pathogenic, also appeared to be polymorphisms in the same database and original source data should be checked [10, 45]. The major differences between the pathogenic mutation and homoplasmic variant sets were that the different homoplasmic variants (a) were located more often in the tRNA loops (41/78 compared with 29/90 for pathogenic mutations), (b) changed less WCs into mismatches in the tRNA stems (19/78 compared with 59/90 for the pathogenic mutations) and (c) affected less conserved base pairs (7/78 compared with 34/90 for the pathogenic mutations). Smaller studies based on database sequences also pointed to these differences between polymorphisms and pathogenic mutations [11, 18, 46]. The relation between conservation and pathogenicity was further demonstrated by evolutionary selection in 14 different primate species [46]. Pathogenic loop and stem mutations were under higher evolutionary pressure than polymorphisms. For the latter, the stem was under greater selective constraint than the loop. Additionally, the mismatches in the TRNA stem were under lower selective constraint than WCs and it has been proposed that mismatch to WC changes might be adaptive in nature [46]. The tRNA secondary structure heat map showed that pathogenic mutations were dominating the $\mathrm{D}$ stem, acceptor stem, anticodon stem and anticodon but the homoplasmic variants showed also some hot spots in these regions. The D loop and T loop were predominantly targeted by homoplasmic variants. Others also showed hotspots for pathogenic mutations in the acceptor and anticodon stems in smaller studies [11]. In contrast, another group [18] reported a random distribution of pathogenic mutations and polymorphisms across the tRNA structure. The different results of the latter publication may be due to the inclusion of different or more 'pathogenic' mutations in their analysis compared with ours. Although the amount of data on tRNA variants in the mtDNA has increased during the past years, absolute conclusions regarding the pathogenicity of mitochondrial tRNA molecules can still not be provided for every variant in those genes.

The rRNA genes showed a frequency of variants in the same range of tRNA genes and codon position 1 and 2 of the protein coding genes. From this and the relatively low number of pathogenic rRNA mutations published, it appears that homoplasmic variants 
in these genes are not tolerated, which would increase the likelihood that a rRNA variant is pathogenic. However, this is in contrast with results from a mice study [43]. There, in offspring from mtDNA mutator mice, purifying selection against nonsynonymous mutations in protein coding genes was evident after two generations. The rRNA and tRNA genes experienced less intense germline selection, comparable with the third codon position in protein coding genes [43]. The difference between the studies is probably due to the inclusion of heteroplasmic mutations in the mice study. The authors suggest that tRNA mutations at low heteroplasmy levels are more compatible with life than some protein coding mutations and are therefore subjected to a less rapid form of purifying selection. Although less rapid, the mechanism of purification seems to be equally important as this study showed comparable selection against variants in tRNAs, rRNAs and first and second codon positions on the population level. Since the rRNA results are comparable with the tRNA results in these studies, the same mechanism might be acting on both, although in contrast to tRNAs not many pathogenic heteroplasmic rRNA mutations have been reported. Examining if heteroplasmy is more tolerated in RNA genes than protein coding genes will give valuable information in the near future.

As expected, the 58 non-coding nucleotides in between the protein coding, rRNA and tRNA genes experienced the lowest selective pressure with the highest number of variants per base pair. This means that even the third codon position of genes is not entirely free of selection. Probably this is due to amino acids that do not allow for synonymous substitution (methionine and tryptophan) or have only limited third codon position possibilities (cysteine, aspartic acid, glutamic acid, phenylalanine, histidine, lysine, asparagine, glutamine, and tyrosine). So, based on functional conservation, it could be expected that for part of the changes on the third codon position selection occurs.

\section{Conclusion}

Factors such as guanine mutation rate and (functional) conservation have been shown to affect the amount and location of homoplasmic variants in the different mitochondrial genes. All coding sequences, including the third codon position, showed evidence of negative selection against variants when compared with the non-coding nucleotides between the genes. This meta analysis provided valuable information for the improvement of pathogenicity criteria and for minimizing the amount of variants that require functional follow-up. However, larger cohorts are still necessary to classify novel variants solely using mutation screening. Extension of our approach in the future, will lead to a detailed map of the mtDNA with regions and positions that are targeted by polymorphisms or pathogenic mutations or never show variation. The latter situation probably points to mutations that are not compatible with life and will never be detected. Altogether, this will provide valuable information for evaluating the pathogenicity of mtDNA variants and counseling families. 


\section{Supplementary data}

Supplementary table 2.1. Subject information (origin and haplogroup).

Supplementary table 2.2. Variants used to determine the haplogroup of the samples.

Supplementary table 2.3. Pathogenic tRNA and protein mtDNA mutations according to the criteria that are listed in the Material and methods.

Supplementary table 2.4. All homoplasmic variants that were detected in 730 subjects according to the revised Cambridge reference sequence.

Supplementary table 2.5. Comparison of the haplogroup distribution of the different cohorts.

Supplementary figure 2.1. Conservation of total gene sequence and variant positions in COII, COIII, ND3 and ATP8 for all codon positions (A) and third codon positions (B).

Supplementary tables are available online at doi:10.1016/j.mito.2011.09.003

\section{References}

1. Anderson, S., et al., Sequence and organization of the human mitochondrial genome. Nature, 1981. 290(5806): p. 457-65.

2. Schmiedel, J., et al., Mitochondrial cytopathies. J Neurol, 2003. 250(3): p. 267-77.

3. DiMauro, S. and E.A. Schon, Mitochondrial respiratory-chain diseases. N Engl J Med, 2003. 348(26): p. 2656-68.

4. Chinnery, P.F. and E.A. Schon, Mitochondria. J Neurol Neurosurg Psychiatry, 2003. 74(9): p. 1188-99.

5. Lucioli, S., et al., Introducing a novel human mtDNA mutation into the Paracoccus denitrificans COX I gene explains functional deficits in a patient. Neurogenetics, 2006. 7(1): p. 51-7.

6. Valente, L., et al., Identification of novel mutations in five patients with mitochondrial encephalomyopathy. Biochim Biophys Acta, 2009. 1787(5): p. 491-501.

7. Elliott, H.R., et al., Pathogenic mitochondrial DNA mutations are common in the general population. Am J Hum Genet, 2008. 83(2): p. 254-60.

8. Jacobs, H.T., Disorders of mitochondrial protein synthesis. Hum Mol Genet, 2003. 12 Spec No 2: p. R293-301.

9. Leveque, M., et al., Whole mitochondrial genome screening in maternally inherited non-syndromic hearing impairment using a microarray resequencing mitochondrial DNA chip. Eur J Hum Genet, 2007. 15(11): p. 1145-55.

10. Bhardwaj, A., et al., MtSNPscore: a combined evidence approach for assessing cumulative impact of mitochondrial variations in disease. BMC Bioinformatics, 2009. 10 Suppl 8: p. S7.

11. McFarland, R., et al., Assigning pathogenicity to mitochondrial tRNA mutations: when "definitely maybe" is not good enough. Trends Genet, 2004. 20(12): p. 591-6.

12. van Den Bosch, B.J., et al., Mutation analysis of the entire mitochondrial genome using denaturing high performance liquid chromatography. Nucleic Acids Res, 2000. 28(20): p. E89.

13. van Eijsden, R.G., et al., Chip-based mtDNA mutation screening enables fast and reliable genetic diagnosis of OXPHOS patients. Genet Med, 2006. 8(10): p. 620-7.

14. White, H.E., et al., Accurate detection and quantitation of heteroplasmic mitochondrial point mutations by pyrosequencing. Genet Test, 2005. 9(3): p. 190-9. 
15. Wong, L.J., T.J. Chen, and D.J. Tan, Detection of mitochondrial DNA mutations using temporal temperature gradient gel electrophoresis. Electrophoresis, 2004. 25(15): p. 2602-10.

16. Xiu-Cheng Fan, A., et al., A rapid and accurate approach to identify single nucleotide polymorphisms of mitochondrial DNA using MALDI-TOF mass spectrometry. Clin Chem Lab Med, 2008. 46(3): p. 299-305.

17. Dobrowolski, S.F., et al., Identifying sequence variants in the human mitochondrial genome using high-resolution melt (HRM) profiling. Hum Mutat, 2009. 30(6): p. 8918.

18. Florentz, C. and M. Sissler, Disease-related versus polymorphic mutations in human mitochondrial tRNAs. Where is the difference? EMBO Rep, 2001. 2(6): p. 481-6.

19. Hartmann, A., et al., Validation of microarray-based resequencing of 93 worldwide mitochondrial genomes. Hum Mutat, 2009. 30(1): p. 115-22.

20. Pandya, G.A., et al., A bioinformatic filter for improved base-call accuracy and polymorphism detection using the Affymetrix GeneChip whole-genome resequencing platform. Nucleic Acids Res, 2007. 35(21): p. e148.

21. Thieme, M., et al., ReseqChip: automated integration of multiple local context probe data from the MitoChip array in mitochondrial DNA sequence assembly. BMC Bioinformatics, 2009. 10: p. 440.

22. Van Eyken, E., et al., Contribution of the $N$-acetyltransferase 2 polymorphism NAT2*6A to age-related hearing impairment. J Med Genet, 2007. 44(9): p. 570-8.

23. Baudouin, S.V., et al., Mitochondrial DNA and survival after sepsis: a prospective study. Lancet, 2005. 366(9503): p. 2118-21.

24. Cutler, D.J., et al., High-throughput variation detection and genotyping using microarrays. Genome Res, 2001. 11(11): p. 1913-25.

25. Mitchell, A.L., et al., Sequence variation in mitochondrial complex I genes: mutation or polymorphism? J Med Genet, 2006. 43(2): p. 175-9.

26. Samuels, D.C., et al., A compositional segmentation of the human mitochondrial genome is related to heterogeneities in the guanine mutation rate. Nucleic Acids Res, 2003. 31(20): p. 6043-52.

27. Helm, M., et al., Search for characteristic structural features of mammalian mitochondrial tRNAs. RNA, 2000. 6(10): p. 1356-79.

28. He, Y., et al., Heteroplasmic mitochondrial DNA mutations in normal and tumour cells. Nature, 2010. 464(7288): p. 610-4.

29. Mithani, S.K., et al., Mitochondrial mutations in adenoid cystic carcinoma of the salivary glands. PLoS One, 2009. 4(12): p. e8493.

30. Rollins, B., et al., Mitochondrial variants in schizophrenia, bipolar disorder, and major depressive disorder. PLoS One, 2009. 4(3): p. e4913.

31. Coller, H.A., et al., High frequency of homoplasmic mitochondrial DNA mutations in human tumors can be explained without selection. Nat Genet, 2001. 28(2): p. 147-50.

32. Kothiyal, P., et al., High-throughput detection of mutations responsible for childhood hearing loss using resequencing microarrays. BMC Biotechnol. 10: p. 10.

33. Schroeder, C., et al., High-throughput resequencing in the diagnosis of BRCA1/2 mutations using oligonucleotide resequencing microarrays. Breast Cancer Res Treat, 2009.

34. Salas, A., et al., mtDNA hypervariable region II (HVII) sequences in human evolution studies. Eur J Hum Genet, 2000. 8(12): p. 964-74.

35. Sigurgardottir, S., et al., The mutation rate in the human mtDNA control region. Am J Hum Genet, 2000. 66(5): p. 1599-609.

36. Galtier, N., et al., Mutation hot spots in mammalian mitochondrial DNA. Genome Res, 2006. 16(2): p. 215-22.

37. Stoneking, M., Hypervariable sites in the mtDNA control region are mutational hotspots. Am J Hum Genet, 2000. 67(4): p. 1029-32. 
38. Tanaka, M. and T. Ozawa, Strand asymmetry in human mitochondrial DNA mutations. Genomics, 1994. 22(2): p. 327-35.

39. Lobry, J.R. and N. Sueoka, Asymmetric directional mutation pressures in bacteria. Genome Biol, 2002. 3(10): p. RESEARCH0058.

40. Faith, J.J. and D.D. Pollock, Likelihood analysis of asymmetrical mutation bias gradients in vertebrate mitochondrial genomes. Genetics, 2003. 165(2): p. 735-45.

41. Green, P., et al., Transcription-associated mutational asymmetry in mammalian evolution. Nat Genet, 2003. 33(4): p. 514-7.

42. Majewski, J., Dependence of mutational asymmetry on gene-expression levels in the human genome. Am J Hum Genet, 2003. 73(3): p. 688-92.

43. Stewart, J.B., et al., Strong purifying selection in transmission of mammalian mitochondrial DNA. PLoS Biol, 2008. 6(1): p. e10.

44. Soares, P., et al., Correcting for purifying selection: an improved human mitochondrial molecular clock. Am J Hum Genet, 2009. 84(6): p. 740-59.

45. Bandelt, H.J., et al., Exaggerated status of "novel" and "pathogenic" mtDNA sequence variants due to inadequate database searches. Hum Mutat, 2009. 30(2): p. 191-6.

46. Ruiz-Pesini, E. and D.C. Wallace, Evidence for adaptive selection acting on the tRNA and rRNA genes of human mitochondrial DNA. Hum Mutat, 2006. 27(11): p. 1072-81. 

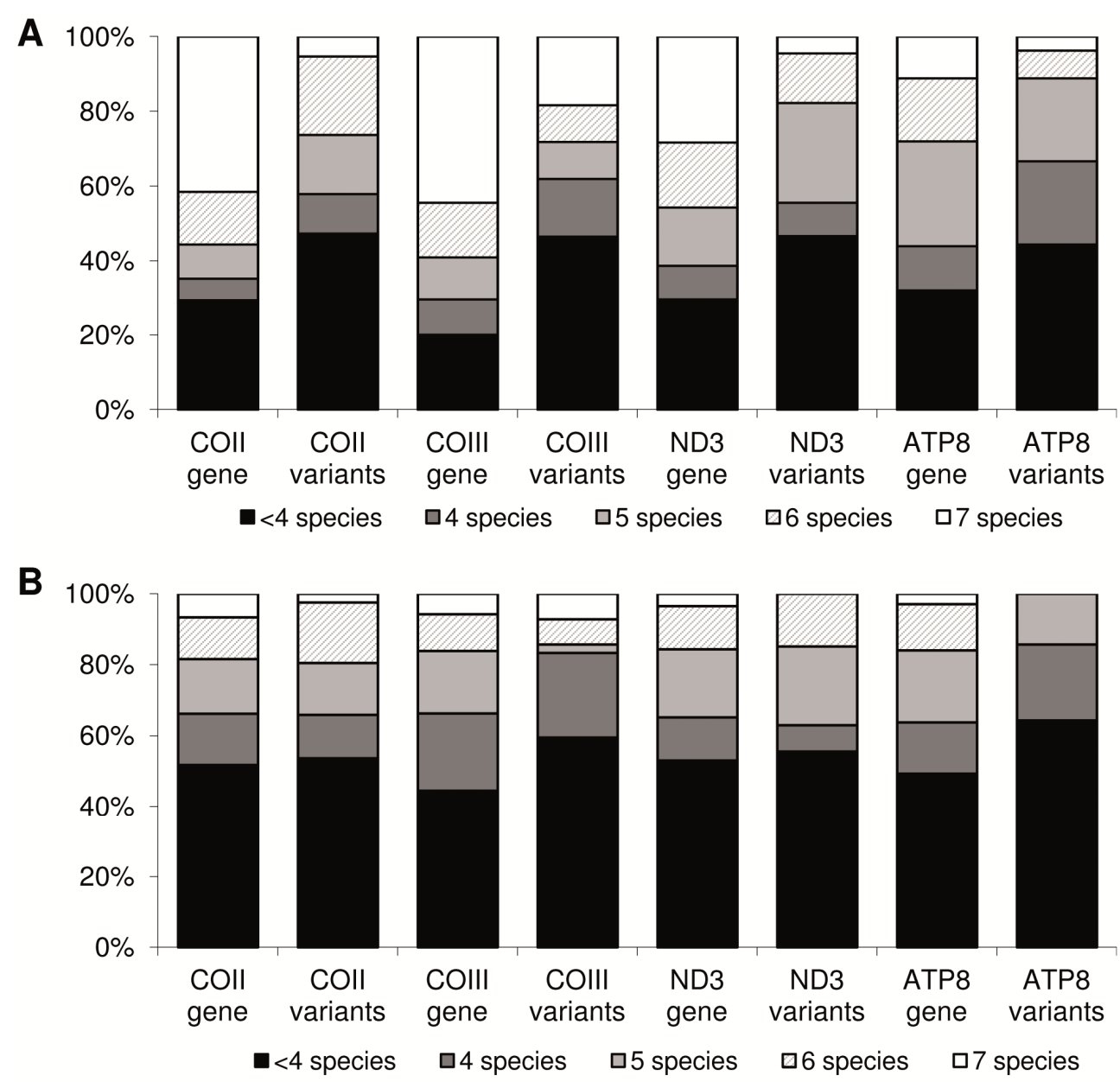

Supplementary figure 2.1. Conservation of total gene sequence and variant positions in COII, COIII, ND3 and ATP8 for all codon positions (A) and third codon positions (B). The human sequence of both genes was aligned with the sequence of six other species: G. gallus, C. lupus, B. taurus, M. musculus, R. norvegicus and D. melanogaster. 



\section{Chapter 3}

POLG1 defects lead to muscle abnormalities, oxidative stress in fibroblasts and apoptosis in brain and liver.

A.M. Voets, B.J.C. van den Bosch, P.J. Lindsey, R.M. Verdijk, F.K. Verheyen, G.C. Schoonderwoerd, S.J.V. Vanherle, J.J. Esseling, P.H.G.M. Willems, W.J.H. Koopman, C.E.M. de Die-Smulders, B.T. Poll-The, M. de Visser, C.G. Faber, I.F.M. de Coo, H.J.M. Smeets

Submitted 


\section{Abstract}

Background. DNA polymerase gamma (pol $\mathrm{y}$ ) is the only polymerase responsible for the replication and maintenance of mitochondrial DNA (mtDNA). Mutations in the catalytic subunit of pol y (POLG1 gene) are associated with a broad variety of clinical symptoms. Fatal complications usually involve brain and liver but muscle abnormalities have also been observed. The pathogenic mechanism of POLG1 mutation is only partly understood. We intend to characterize molecular pathways in POLG1 pathology.

Methods. Light and electron microscopy, mtDNA copy number and rearrangements and whole genome gene expression (Affymetrix) profiles were evaluated in skeletal muscle biopsies of POLG1 patients. Apoptosis and oxidative stress were examined in different human tissues.

Results. Most patients showed decreased mtDNA copy number, accumulation of mtDNA deletions and/or decreased OXPHOS activity in skeletal muscle. Morphological changes were observed in the majority of patients' skeletal muscles biopsies. Transcriptomics revealed lower expression of fatty acid beta oxidation, increased expression of electron transport chain subunits and decreased expression of uncoupling proteins 2 and 3 . Increased apoptosis was shown in the brain and liver and increased oxidative stress was measured in fibroblast cultures in part of the patients.

Conclusion. Except for two patients, all POLG1 patients showed muscle abnormalities for at least one of the investigated parameters, indicating that any of these observations can be suggestive for defects in POLG1. Oxidative stress and apoptosis were identified as pathological processes in part of the patients and may be counterbalanced by a genetic-background-dependent yet unidentified mechanism.

\section{Key words}

POLG1, oxidative stress, apoptosis, gene expression 


\section{Introduction}

Mitochondrial diseases are among the most frequently inherited neurological disorders [1]. DNA polymerase gamma ( $\mathrm{pol} \mathrm{y})$ is the sole DNA polymerase involved in the replication and maintenance of the mtDNA. POLG1 mutations have been reported to cause a broad variety of phenotypes, such as Alpers-Huttenlocher syndrome, ataxia neuropathy syndrome (ANS), epilepsy and chronic progressive external ophtalmoplegia (CPEO) [2]. Phenotypes involving hepatopathy usually manifest early in life, while isolated myopathy affects older individuals and brain abnormalities can occur at any age [3]. The correlation between genotype and phenotype in patients with POLG1 mutations is sometimes tight but more often variable [3]. The pathological mechanism explaining the diverse clinical manifestations of POLG1 mutations is only partly understood. Human studies have focused on in vitro characterization of purified or recombinant enzyme [4], in vivo analysis of the functions of the pol y protein and its domains in cultured cells [5] and large scale screening of mtDNA molecules in skeletal muscle and fibroblasts [6, 7]. These studies showed a higher probability of accumulating mtDNA mutations and/or depletion of mtDNA when POLG1 is mutated.

The aim of this study is to identify molecular mechanisms involved in human POLG1 pathogenesis by examining skeletal muscle, fibroblast cultures and (post mortem) liver and brain specimens of POLG1 patients. This is the first study combining gene expression analysis with physiological measurements and staining in human patients. Identification of pathology associated processes is important to decipher the clinical heterogeneity, to identify new treatment approaches and improve prognosis.

\section{Material and methods}

\section{POLG1 patients}

Characteristics of eleven patients homozygous or compound heterozygous for POLG1 mutations are described in table 3.1. Blood lactate levels of the patients at rest and enzyme activities of the OXPHOS complexes derived from muscle biopsies are presented when available (table 3.1). Not all assays could be performed for all patients due to the limited material available. Ethics approval was not required according to Dutch legislation as residual material of previous diagnostic investigations has been studied.

\section{Skeletal muscle morphology}

Muscle biopsies were taken from the quadriceps muscle. Samples were split for light microscopy (LM) and electron microscopy (EM). Skeletal muscle histology - HE (haematoxolin and eosin), trichrome, periodic acid-Schiff (PAS) and ORO (oil red O) staining - and enzyme histochemistry - acid phosphatase, ATPase (adenosine triphosphatase), NADH (nicotinamide adenine dinucleotide), SDH (succinate dehydrogenase) and COX (cytochrome oxidase) - were evaluated. Ultrastructural studies were performed as described previously [8]. 
Mitochondrial DNA copy number and large scale deletions

DNA was isolated using the Wizard Genomic DNA Purification Kit (Promega, Leiden, $\mathrm{NL}$ ). Mitochondrial DNA copy number was determined as described previously [8]. To detect mtDNA deletions, $50 \mathrm{ng}$ of DNA was amplified into two overlapping fragments of 16.1 (I) and 16.0 (II) kb using Phusion Hot Start DNA polymerase (Finnzymes) and 100 ng forward and reverse primer corresponded to nucleotide positions (nt, 5' to 3', according to Cambridge sequence) Fwdl 314-343 ; Revl 16382- 16411; Fwdll 13301355; Revll 756- 778). The PCR conditions were as follows : $30 \mathrm{sec}$ at $98^{\circ} \mathrm{C} ; 30$ cycles of $10 \mathrm{sec}$ at $98^{\circ} \mathrm{C}$ and $8 \mathrm{~min} 15 \mathrm{sec}$ at $72^{\circ} \mathrm{C} ; 10 \mathrm{~min}$ at $72^{\circ} \mathrm{C}$. PCR products were first analyzed on a $1 \%$ agarose gel and next for $32 \mathrm{~h}$ on a $0.7 \%$ agarose gel.

\section{Microarray procedure}

Skeletal muscle needle biopsies were immediately frozen in liquid nitrogen from six subjects carrying a POLG1 mutation and twelve controls (table 3.2). Total RNA was isolated using the TRIzol reagent (Invitrogen, Carlsbad, CA, USA) and purified with the RNeasy clean-up kit (Qiagen, Hilden, DE). RNA quantity and purity were determined spectrophotometrically using the Nanodrop ND-1000 (Nanodrop Technologies) and RNA integrity was assessed by determining the RNA 28S/18S ratio using the Bioanalyser 2100 (Agilent Technologies, Santa Clara, CA, USA). $150 \mathrm{ng}$ of muscle RNA was reverse transcribed into CDNA and amplified in a two-round amplification reaction according to the manufacturer's protocol (Affymetrix, Santa Clara, CA, USA). A mixture of cDNA and added hybridization controls was hybridized on Affymetrix HGU133 Plus 2.0 chips, followed by staining and washing steps in the GeneChip fluidics station 400 (Affymetrix) according to the manufacturer's procedures. To assess the raw probe signal intensities, chips were scanned using the GeneChip scanner 3000 (Affymetrix).

\section{Microarray data analysis}

Images of the Human Genome U133 Plus 2.0 arrays were quantified with GCOS software (Affymetrix). The microarray data reported in this manuscript have been deposited in NCBI Gene expression omnibus (GEO), accession number GSE18715. The chip description file (CDF) used for the analysis was an update created and freely distributed by the microarray lab of the university of Michigan (http://brainarray.mbni.med.umich.edu; [9]) based on UniGenes (version 9). This resulted in the analysis of 17215 gene-transcripts out of the 54613 commonly obtained using the Human Genome U133 Plus 2.0 CDF provided by Affymetrix. All genes were analyzed using a Gaussian linear regression $\left(N\left(\mu, \sigma^{2}\right)\right.$ where $\mu$ is the mean and $\sigma^{2}$ is the variance) including the best hybridization and best labeling spike, the age, and sex. The inference criterion used for comparing the models is their ability to predict the observed data, i.e. models are compared directly through their minimized minus loglikelihood. When the numbers of parameters in models differ, they are penalized by adding the number of estimated parameters, a form of the Akaike information criterion (AIC) [10]. For each gene, a model containing the relevant covariates $(E(y)=$ Hyb. Spike + Lab. Spike + Age + Sex) was fitted in order to obtain a reference AIC. Then a model containing the group was fitted $(E(y)=$ Hyb. Spike + Lab. Spike + Age + Sex + 
Grp). The gene under consideration was found to be differentially expressed if the AIC of this second model was smaller when compared to the AIC of the model not containing the group effect. The genes analyzed and fold changes were loaded into GenMapp (version 2.1, build 20080507) and MAPPFinder (version 2.0, build 20041218) software packages to evaluate the transcripts in relation to known biological processes. The gene database version "Hs-Std_20070817" and the Mapps version "Hs_Contributed_20080619" were used for both programs. Only gene-transcripts with either their average intensities for the control and patient groups above 500 or average intensities for one of these groups above 1000 (background signal criteria) and a 10 percent fold change were used to obtain a ranked list of pathways with differentially expressed genes.

\section{Quantification of reactive oxygen species}

Reactive oxygen species (ROS) levels were measured in four patient (P54, P194, P382 and P504) and three control fibroblast cultures by dihydroethidine (DHE) oxidation as described previously [11]. All cell lines were cultured in parallel. Similarly to the microarray data, the ROS data were analyzed using a Gaussian linear regression including the day, medium, group, and medium-group interactions if required. As previously, the Akaike Information Criteria (AIC) was used to compare models without and with the group effect. The model with the smallest AIC was selected as the model best fitting the observed data.

\section{Histology and apoptosis in liver and brain}

Histological and immunohistochemical analysis was performed on formalin fixed paraffin embedded sections $(4 \mu \mathrm{m})$ of liver and brain of patients (P30, P382 and P504) and controls. Anonymous control liver $(n=3)$ and brain $(n=3)$ sections (age 15 or older) were used for comparison. Subsequent sections were used for HE staining and the detection of apoptosis using immunohistochemistry (IHC) with rabbit-anti cleaved caspase 3 primary antibody (Cell Signalling, Danvers, MA, USA). IHC was performed according to the manufacturer's protocol. In short, endogenous peroxidase was blocked by incubation with $0.3 \% \mathrm{H}_{2} \mathrm{O}_{2}$ in methanol for 20 minutes, tissue antigenicity was recovered using heat-based retrieval $\left(10 \mathrm{mM}\right.$ citrate buffer, $\mathrm{pH} 6,95^{\circ} \mathrm{C}, 10 \mathrm{~min}$ ), sections were blocked with $4 \%$ normal swine serum (DAKO) in phosphate buffered saline containing $1 \%$ BSA and $0.1 \%$ tween (PBT) and incubated overnight with 1:100 primary antibody in $5 \%$ foetal calve serum in PBT. After washing, 1:1000 biotinconjugated swine anti-rabbit secondary antibody (DAKO) in PBT was applied for 30 minutes, followed by 30 minutes of Vectastain $A B C$ reagent (Vector Laboratories, Burlingame, CA, USA). 3'3-diaminobenzidine (DAB, DAKO) was applied as a color substrate and hematoxylin for nuclear counterstaining. Sections were dehydrated in a graded ethanol solution and xylene, subsequently enclosed with Entellan (Merck) and evaluated with a Nikon Eclipse E800 microscope. Pictures were taken with a Nikon Digital Camera DXM 1200F and analyzed using the Lucia G version 4.81 (Laboratory Imaging) software. Cleaved caspase 3 positive (apoptotic) cells were counted in six random pictures and expressed as percentage of all cells. 


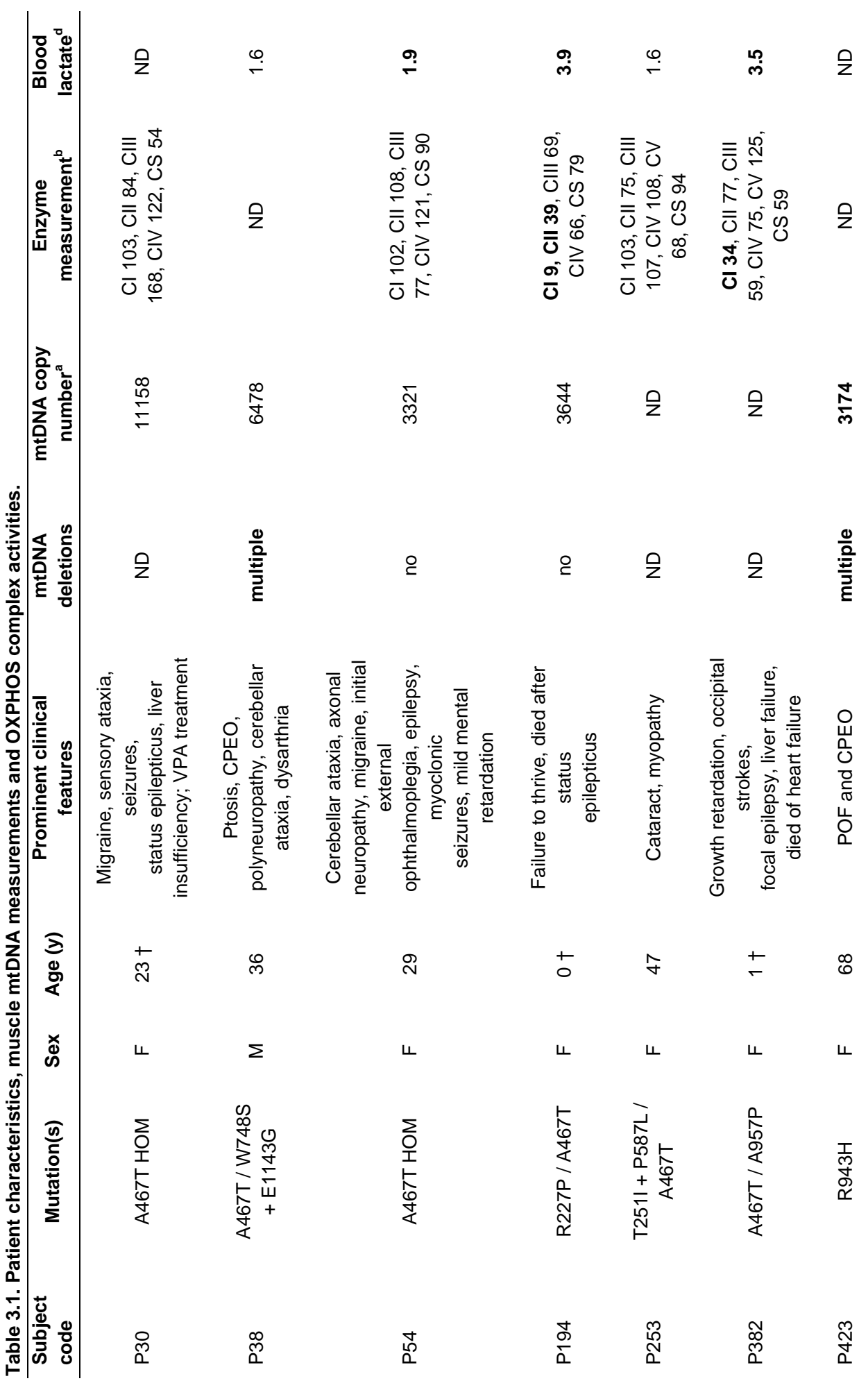




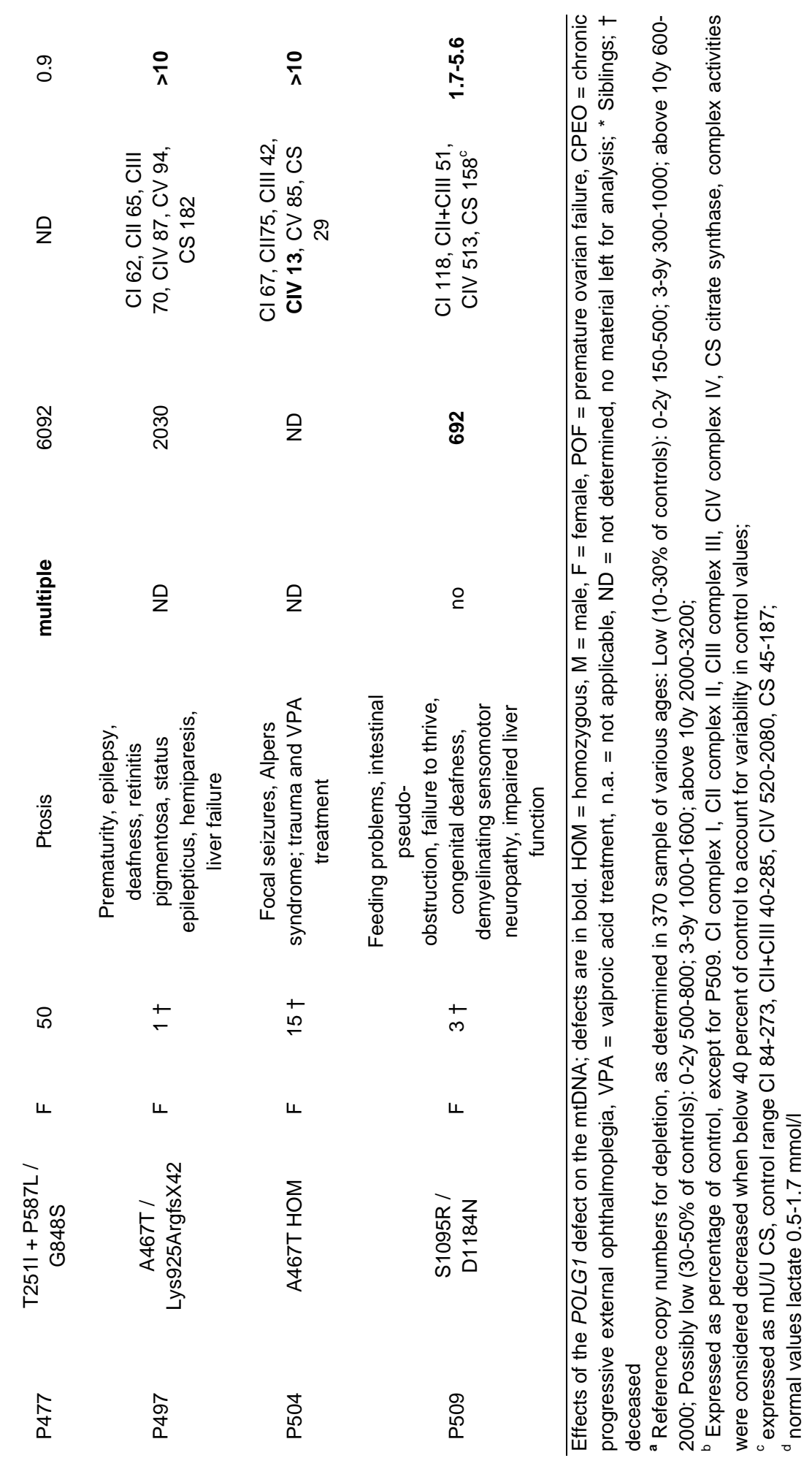




\section{Results}

In total, eleven patients with POLG1 mutations were included in the study (table 3.1). Three patients were homozygous for p.A467T, five patients were compound heterozygous for this mutation and another mutation, two patients were compound heterozygote for other mutations and one patient had an autosomal dominant mutation (P423). Eight patients presented with neurological problems, five patients suffered from impaired liver function and three patients had chronic PEO and/or ptosis. Four patients died within their first years of life, one patient (P504) was diagnosed incidentally after death of valproic acid (VPA) treatment of post traumatic epileptic seizures and one patient (P30) experienced clinical symptoms for a prolonged time but deceased after VPA treatment. Based on these data, no obvious genotype-phenotype association could be observed.

\section{Muscle mtDNA and morphology}

In total, four out of eight patients showed either reduced mtDNA copy number (P509) or the presence of multiple deletions in skeletal muscle (P38, P423 and P477, table 3.1). Normal values were observed in the younger patients ageing 0 to 23 years. In three out of eight patients the activity of one or more OXPHOS complexes was decreased in muscle biopsies (table 3.1). Seven out of eight patients showed morphologic abnormalities in muscle. Four patients showed myopathic changes with ragged red fibers and multiple cytochrome $c$ oxidase negative muscle fibers (patients P38, P194, P253 and P509; figure 3.1A-C). Another three patients (P497, P382 and P504) exhibited a striped positive staining of the muscle fibers in the trichrome stain in the absence of ragged red fibers. One patient (P54) did not show any changes of the muscle morphology. Ultrastructural changes were observed in all five patients (P194, P253, P497, P504 and P509) evaluated on electron microscopy with aberrant shape, size and distribution of the mitochondria. The mitochondria appeared elongated in shape or rounded with tubular cristae. Mitochondria were frequently aggregated and localized in the subsarcolemmal and A-band areas. One in five patients showed mitochondria with paracristalline inclusions (patient P253; figure 3.1D).

\section{Muscle gene expression analysis}

Gene expression levels of 17,215 transcripts were measured in skeletal muscle samples of six POLG1 patients and twelve age matched controls (table 3.2). Transcripts of 3,718 genes passed the background signal criteria. The expression of 1,193 transcripts was significantly different between controls and POLG1 patients (Appendix). Of the differentially expressed genes, 247 transcripts were increased and 946 were decreased, respectively. These results indicated mostly small (fold change < 1.5) gene expression changes. QPCR confirmed nine out of ten microarray results (data not shown). 

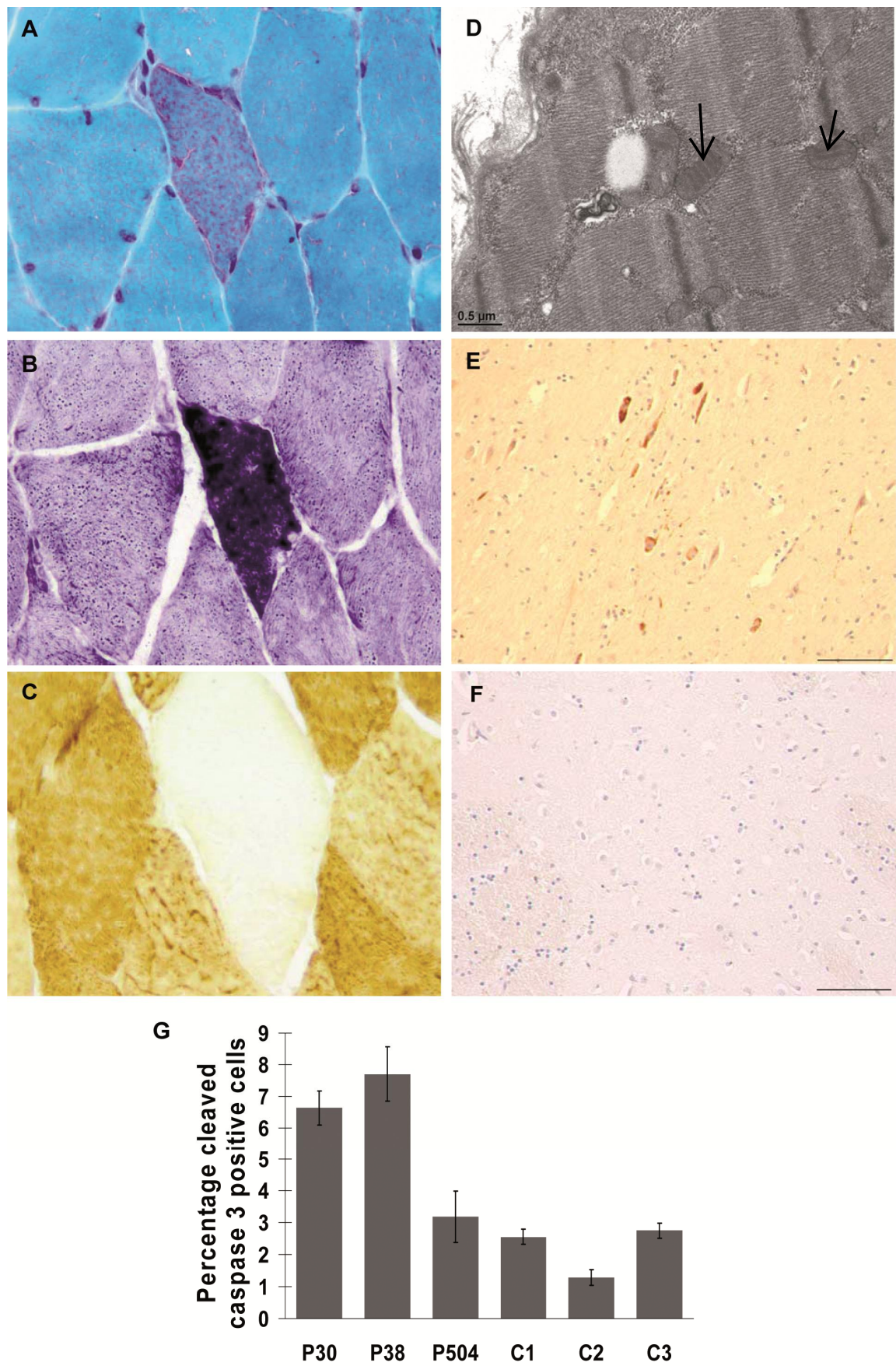

Figure 3.1. Histology and apoptosis in tissues of POLG1 patients. Muscle biopsy: Modified Gomori trichrome (A), NADH (B) and cytochrome c oxidase (C) stain (400x) and electron microscopy (D). Clustered apoptotic cells in the brain of patient P30 (E), which are absent in controls (F) and the summarized results of apoptosis detection in liver of patients $(P)$ and controls $(C)$ (G). Bars in $E$ and $F$ are $100 \mu \mathrm{m}$. Arrows in panel $\mathrm{D}$ indicate paracristalline inclusions. 
Table 3.2. Patients and controls for gene expression analysis.

\begin{tabular}{|c|c|c|c|c|c|}
\hline Patient code & Sex & Age at biopsy & Control code & Sex & Age at biopsy \\
\hline \multicolumn{6}{|c|}{ Age group: young } \\
\hline P194 & $\mathrm{F}$ & 0 & $\mathrm{C} 11$ & $\mathrm{M}$ & 0 \\
\hline \multirow[t]{5}{*}{ P509 } & $\mathrm{F}$ & 3 & $\mathrm{C} 12$ & M & 1 \\
\hline & & & $\mathrm{C} 13$ & M & 9 \\
\hline & & & $\mathrm{C} 14$ & M & 2 \\
\hline & & & C15 & $\mathrm{F}$ & 5 \\
\hline & & & $\mathrm{C} 16$ & M & 2 \\
\hline \multicolumn{6}{|c|}{ Age group: middle } \\
\hline P38 & M & 36 & C3 & $\mathrm{F}$ & 34 \\
\hline \multirow[t]{3}{*}{ P54 } & $\mathrm{F}$ & 29 & C5 & $\mathrm{F}$ & 17 \\
\hline & & & $\mathrm{C} 7$ & $\mathrm{~F}$ & 22 \\
\hline & & & C9 & M & 25 \\
\hline \multicolumn{6}{|l|}{ Age group: old } \\
\hline P423 & $\mathrm{F}$ & 68 & C4 & $\mathrm{F}$ & 64 \\
\hline P477 & $\mathrm{F}$ & 50 & C8 & $M$ & 53 \\
\hline
\end{tabular}

Overview of patients and controls used for the microarray gene expression analysis. $\mathrm{P}=$ patient, $\mathrm{C}=$ control, $\mathrm{M}=$ male, $\mathrm{F}=$ female; ${ }^{*}$ Age groups: young $(0-10$ years old $)$, middle $(11-49$ years old $)$, old $(\geq$ 50 years old)

Pathway analysis, using MAPPFinder, showed ten significantly altered pathways $(p<0.05$; table 3.3$)$. In patient cells, the electron transport chain showed higher gene expression levels for $75 \%$ of its differentially expressed transcripts, whereas uncoupling protein 2 (UCP2) transcripts were decreased. In contrast, the expression of almost all differentially expressed genes in the fatty acid beta oxidation pathway was decreased (table 3.3). Transcripts of carnitine palmitoyltransferase 1 (CPT1) $\mathrm{B}$, which is the ratelimiting step in beta oxidation, were decreased. This indicated reduced fatty acid catabolism. In the triglyceride synthesis pathway, the expression of most changed genes was decreased (table 3.3). Gene expression levels of $\mathrm{NAD}^{+}$dependent enzymes SIRT2 and poly-(ADP-ribose) polymerase 1 (PARP1) were decreased (both fold change 0.79). Based on the observed changes, we performed an additional QPCR for SIRT1 and UCP3, which had intensity levels in the background range of the microarray analysis. In the QPCR analysis, both were significantly decreased with fold changes 0.74 and 0.43 , respectively. POLG1 itself also showed intensity levels in the background range.

Overall, oxidative stress or apoptosis pathways were not significantly altered in muscle of POLG1 patients. Nevertheless, in the oxidative stress pathway, superoxide dismutase 1 (SOD1) and glutathione peroxidase 4 (GPX4) gene expression levels were changed significantly with fold changes 0.91 and 1.46 , respectively. Many genes in the apoptosis pathway did not reach significance due to low signal intensities. However, for the expression of 31 genes there was a tendency to be increased $(n=16)$ or decreased $(n=15)$ with an inconclusive effect on the total pathway. There were no significant 
changes in the expression level of genes involved in mitochondrial biogenesis peroxisome proliferator-activated receptor gamma coactivator 1-alpha/beta $(P G C 1-\alpha / \beta)$, nuclear respiratory factor 1 (NRF-1) and mitochondrial transcription factor A (TFAM). Only minor changes were observed in the expression of genes involved in mitochondrial fusion and fission (OPA1 fold change 0.81 and MFN2 fold change 0.83 ).

Table 3.3. MAPPFinder results grouped into related categories.

\begin{tabular}{lcccccc}
\hline MAPP Name & Changed & Up & Down & Measured & On MAPP & P-value \\
\hline Metabolism and energy production & & & & & & \\
Electron Transport Chain & 20 & 15 & 5 & 97 & 105 & 0.000 \\
Triglyceride Synthesis & 9 & 2 & 7 & 23 & 24 & 0.000 \\
Fatty Acid Beta Oxidation Meta & 7 & 1 & 6 & 32 & 32 & 0.020 \\
& & & & & & \\
Transcription/translation & 29 & 10 & 19 & 119 & 127 & 0.000 \\
mRNA processing Reactome & 12 & 1 & 11 & 42 & 50 & 0.000 \\
Translation Factors & 9 & 2 & 7 & 36 & 40 & 0.008 \\
RNA transcription Reactome & & & & & & \\
& & & & & & \\
Others & 6 & 0 & 6 & 23 & 24 & 0.012 \\
IL-9 NetPath 20 & 4 & 1 & 3 & 16 & 17 & 0.043 \\
Nucleotide Metabolism & 8 & 1 & 7 & 46 & 48 & 0.046 \\
Circadian Exercise & 7 & 1 & 6 & 38 & 38 & 0.048 \\
Striated Muscle Contraction & & & & & & \\
\hline Number of differentily expressed
\end{tabular}

Number of differentially expressed genes in the significantly changed $(p<0.05)$ pathways according to MAPPFinder.

Increased ROS levels in POLG1 patient fibroblasts

Reactive oxygen species (ROS) levels were significantly increased in fibroblasts of patients P54, P194 and P382 (136\% (confidence interval (c.i.) 114-163), 117\% (c.i. 104-133) and $251 \%$ (c.i. 213-295) of controls, respectively), but not of patient P504 (112\% of controls (c.i. $96-130))$.

\section{Histology and apoptosis in liver and brain}

Histology of liver specimens of three investigated patients (P504, P382 and P30) showed a microvesicular steatosis with marked fibrosis, ductular proliferation and cholestasis as described before [12]. Hepatocyte mitochondria of patient P509 appeared aberrant in shape and size and often contained crystalloid inclusions. Post mortem examination of the brain of patient P504, exhibited typical Alpers-Huttenlocher like changes with ischemia and gliosis of the occipital cortex combined with spongiosis and gliosis of cortical and deep gray matter regions with a subtle and generalized decrease of neuronal numbers. The brain of patient P30 on the other hand did not exhibit changes of the occipital cortex. In this patient a degeneration with decrease of neuronal numbers and gliosis at all levels of the corticospinal tracts was observed, compatible with a phenotype of Friedreichs ataxia. Immunohistochemistry identified a 
higher percentage of cleaved caspase 3 positive cells in liver of two (2/3) POLG1 patients compared with three controls (figure 3.1G). In POLG1 brain sections, a few apoptotic hot spots could be seen (figure 3.1E), which were not present in control brain sections (figure 3.1F).

\section{Discussion}

This study provides the first comprehensive characterization of molecular genetic, biochemical and morphological processes in human POLG1 patients.

\section{Muscle abnormalities in POLG1 patients}

This study revealed variable muscle aberrations in virtually all POLG1 patients investigated. All patients tested showed ultrastructural changes on electron microscopy, which is in this study the most sensitive test. Other aberrations were related to the mtDNA (multiple deletions or decreased copy number), the activity of OXPHOS enzymes or were morphological changes. The three adult POLG1 patients over 35 years old, for which mtDNA rearrangements could be analyzed, showed multiple deletions. Decreased activity of respiratory chain enzymes was observed in three out of eight patients. The presence of multiple deletions, mtDNA depletion or decreased OXPHOS function is suggestive for defects in the mtDNA maintenance and POLG1, but the absence of these features does not exclude mtDNA maintenance or POLG1 defects, especially in young patients (P30 and P54 [13]). Nevertheless, because muscle biopsies will not always be available and based on clinical manifestations POLG1 sequencing can be the first step, especially if an urgent diagnosis is required for treatment.

\section{Changes in tissues of POLG1 patients}

\section{Metabolic switch in skeletal muscle}

Transcription of genes in the beta oxidation pathway was significantly decreased. This might be explained by increased NADH levels as previously observed in fibroblasts from patients with isolated mitochondrial complex I (CI) deficiency [14]. Three of the patients in this study indeed showed a decreased OXPHOS function. Decreased OXPHOS-derived ATP production can stimulate glycolysis. This is associated with higher lactate production, inhibition of carnitine palmitoyltransferase 1 (CPT1) and thus also beta oxidation [15] (figure 3.2). Excess NADH in the cytosol can be oxidized by lactate dehydrogenase. Increased lactate levels are therefore often measured in patients with mitochondrial disease, including POLG1 patients [16]. In this study, six out of nine patients showed increased lactate levels in serum. The increased transcription of the OXPHOS complexes could be a rescue attempt to process excess NADH and supply the cell of sufficient energy. This is supported by the decreased transcription of UCP2 and UCP3 leading to increased coupling of proton pumping and ATP production. The expected higher levels of point mutations and deletions in the mtDNA associated with defect pol y [5] will only allow for increased quantity but not quality of the OXPHOS subunits when their transcription is increased. Therefore, the compensatory mechanism 
might only partially be effective in tissues in which the OXPHOS system is not maximally used (e.g. fibroblasts), but would not meet the energy demand of highly active tissues such as muscle, liver and brain, leading to disease symptoms.

In this study, no significant effect was detected on mitochondrial or mtDNA biogenesis and its key regulator PGC1- $\alpha$, even though two patients showed decreased mtDNA copy number. However, SIRT1 transcripts were decreased. SIRT1 normally stimulates mitochondrial biogenesis and fatty acid $\beta$ oxidation through post-translational activation of PGC1- $\alpha$ by deacetylation [17]. The $\mathrm{NAD}^{+} / \mathrm{NADH}$ ratio has been shown to regulate transcription of the SIRT1 gene [17], emphasizing additional cellular consequences of disturbed NADH metabolism.

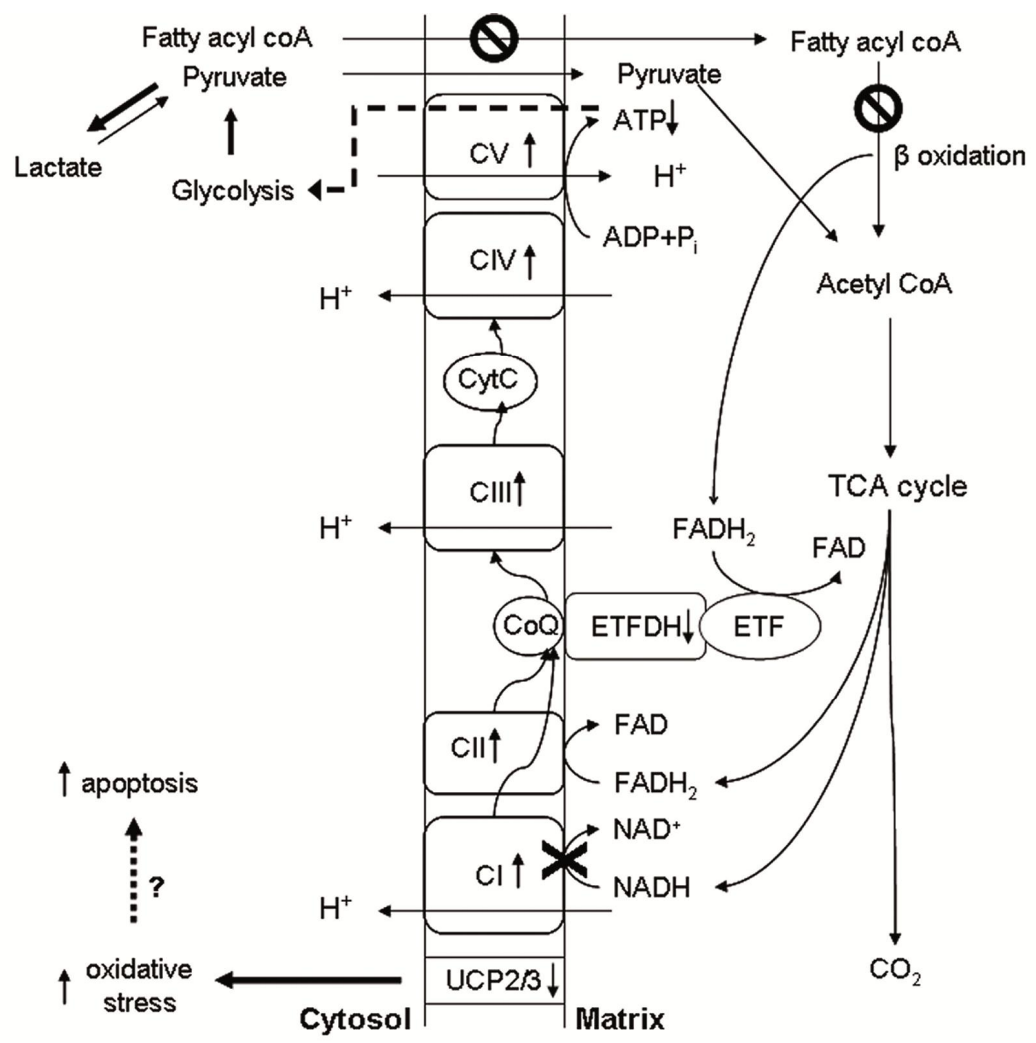

Figure 3.2. Pathophysiological processes in POLG1 patients. Processes differentially expressed in POLG1patients: decreased beta oxidation, increased expression of OXPHOS subunits and decreased expression of uncoupling proteins. These changes suggest increased glycolysis, oxidative stress and apoptosis.

\section{Mitochondrial fusion and fission}

Only two genes associated with mitochondrial fusion and fission (OPA1 and MFN2) showed minor gene expression changes. Recent literature states that mitochondrial fusion is essential for the equilibration of nuclear-encoded mitochondrial proteins over all organelles but leads to clonal expansion of mitochondrial deletion mutants with replicative advantage. Therefore, fusion is normally coupled to fission which enables 
selective degradation of damaged mitochondria with decreased membrane potential by mitophagy [18]. The gene expression analysis does not provide convincing evidence for a role of mitochondrial fission and fusion in POLG1 pathology. However, given the posttranslational regulation of these processes [19], this should still be investigated on the protein level when patient material is available.

\section{Increased ROS levels in fibroblast cultures}

The involvement of oxidative stress in POLG1 pathology is controversial. The overall oxidative stress pathway expression was not significantly changed, but particular genes such as SOD1 and GPX4 transcripts showed altered expression levels. Also UCP3 expression was decreased. In the setting of doxorubicin-induced heart failure in rats, compensatory down-regulation of myocardial UCP2 and UCP3 lead to increased mitochondrial ROS production [20]. ROS levels were measured in fibroblast cultures of four patients and found to be increased in three (P54, P194 and P382) of them compared with controls, but not in patient P504. Increased ROS levels in OXPHOS disease are usually believed to originate from complex I or III sites. However, only fibroblasts of P194 showed a significant biochemical defect (data not shown). The method used cannot distinguish the exact cellular localization of hydroethidine oxidation [11], thus, another explanation for increased ROS levels could be the activation of alpha-ketoglutarate dehydrogenase in the tricarboxylic acid cycle. This enzyme can be stimulated to produce ROS by increased NADH levels [21]. The fact that one patient with and another patient without oxidative stress are both homozygous for the p.A467T mutation implies that it is not only the POLG defect that triggers ROS production. The patients may differ in the mtDNA defects resulting from the POLG1 mutations, but we could not test this, because no muscle was left for one of the patients (P504). On the other hand, other genetic (protective or exacerbating) factors could be involved in controlling ROS levels. Altogether, the current results correspond with the unclear and controversial involvement of ROS in POLG1 pathogenesis today.

Different transgenic mouse models are discordant on the presence [22] or absence [23] of increased oxidative stress due to pol y defects. Furthermore, increased oxidative protein damage in the heart of these mice could be reduced by cardial mCAT overexpression, leading to attenuated cardiomyopathy [22]. Our results in human patients indicated that oxidative stress probably plays a role in part, but not all, of the POLG1 patients. Divergent results in the different mouse models and patients could possibly be related to alternative effects of different POLG1 mutations, mtDNA defects or different genetic backgrounds (i.e. antioxidant expression levels).

\section{Apoptosis in liver and brain}

The expression of only few apoptosis-related genes was significantly changed in muscle. Nevertheless, multiple other apoptosis genes showed a trend towards altered expression. Therefore, the presence of apoptotic cells in liver and brain, the most severely affected tissues in POLG1 patients, was assessed. In the liver, apoptosis was distributed relatively evenly across the section and two out of three patients showed a more than twofold increase of apoptotic cells compared with controls. Different from the liver, there were only a few clusters of apoptotic cells in the brain of patients but not of 
controls. In agreement, results from transgenic POLG mice showed the induction of apoptosis in the duodenum, liver, testis, thymus and skeletal muscle by measuring caspase 3 cleavage and TUNEL staining [23]. Strikingly, the two patients with increased liver apoptosis (P30 and P382) also showed increased oxidative stress in fibroblasts cultures, whereas both were not seen in the third patient (P504). This may suggest that apoptosis, possibly associated with increased ROS levels, contributes to POLG1 related pathology (P30 and P382). However, the acute effect of trauma or drugs (e.g. VPA treatment) seems a much stronger trigger of acute organ failure in a previously disease-free individual (P504).

\section{Conclusions}

Even though muscle, liver and brain material of POLG1 patients is scarce, this study elucidated a number of processes involved in POLG1 pathogenesis. The heterogeneity in clinical presentation is reflected in the variable morphological, molecular and biochemical findings that were obtained. Increased oxidative stress and increased apoptosis appear to be related to the severity of the phenotype although trauma and/or drugs can shortcut this pathological route and lead to acute organ failure. In the future, measuring oxidative damage and apoptosis in liver and brain of additional patients is necessary to establish the link between ROS levels and apoptosis induction.

\section{Acknowledgements}

This work was supported by the Dutch IOP Genomics grant IGE5003C1 and the Kerry foundation (Maastricht, the Netherlands). We would like to thank A. Hendrickx, G. Konings, R. Kamps and M. Gijbels for technical expertise and help and H.R. Scholte for carefully reading the manuscript.

\section{Supplementary material}

Supplementary table 1 (in Appendix) - Significantly differentially expressed genes in muscle of POLG1 patients

\section{References}

1. McFarland, R., R.W. Taylor, and D.M. Turnbull, A neurological perspective on mitochondrial disease. Lancet Neurol, 2010. 9(8): p. 829-40.

2. Wong, L.J., et al., Molecular and clinical genetics of mitochondrial diseases due to POLG mutations. Hum Mutat, 2008. 29(9): p. E150-E172.

3. Milone, M. and R. Massie, Polymerase gamma 1 mutations: clinical correlations. Neurologist, 2010. 16(2): p. 84-91. 
4. Chan, S.S. and W.C. Copeland, Functional analysis of mutant mitochondrial DNA polymerase proteins involved in human disease. Methods Mol Biol, 2009. 554: p. 5972.

5. Spelbrink, J.N., et al., In vivo functional analysis of the human mitochondrial DNA polymerase POLG expressed in cultured human cells. J Biol Chem, 2000. 275(32): p. 24818-28.

6. Ashley, N., et al., Depletion of mitochondrial DNA in fibroblast cultures from patients with POLG1 mutations is a consequence of catalytic mutations. Hum Mol Genet, 2008. 17(16): p. 2496-506.

7. Del Bo, R., et al., Remarkable infidelity of polymerase gammaA associated with mutations in POLG1 exonuclease domain. Neurology, 2003. 61(7): p. 903-8.

8. van Tienen, F.H., et al., Prolonged Nrf1 overexpression triggers adipocyte inflammation and insulin resistance. J Cell Biochem, 2010. 111(6): p. 1575-85.

9. Dai, M., et al., Evolving gene/transcript definitions significantly alter the interpretation of GeneChip data. Nucleic Acids Res, 2005. 33(20): p. e175.

10. Akaike, H. Information theory and an extension of the maximum likelihood principle. in Second International Symposium on Inference Theory. 1973. Budapest: Akadémiai Kiadó.

11. Verkaart, S., et al., Superoxide production is inversely related to complex I activity in inherited complex I deficiency. Biochim Biophys Acta, 2007. 1772(3): p. 373-81.

12. Muller-Hocker, J., et al., Mitochondrial DNA depletion and fatal infantile hepatic failure due to mutations in the mitochondrial polymerase gamma (POLG) gene $A$ combined morphological/enzyme histochemical and immunocytochemical/biochemical and molecular genetic study. J Cell Mol Med, 2009.

13. Blok, M.J., et al., The unfolding clinical spectrum of POLG mutations. J Med Genet, 2009. 46(11): p. 776-85.

14. Verkaart, S., et al., Mitochondrial and cytosolic thiol redox state are not detectably altered in isolated human NADH:ubiquinone oxidoreductase deficiency. Biochim Biophys Acta, 2007. 1772(9): p. 1041-51.

15. Sahlin, K., et al., Turning down lipid oxidation during heavy exercise--what is the mechanism? J Physiol Pharmacol, 2008. 59 Suppl 7: p. 19-30.

16. de Vries, M.C., et al., Multiple oxidative phosphorylation deficiencies in severe childhood multi-system disorders due to polymerase gamma (POLG1) mutations. Eur J Pediatr, 2007. 166(3): p. 229-34.

17. Fulco, M. and V. Sartorelli, Comparing and contrasting the roles of AMPK and SIRT1 in metabolic tissues. Cell Cycle, 2008. 7(23): p. 3669-79.

18. Kowald, A. and T.B. Kirkwood, Evolution of the mitochondrial fusion-fission cycle and its role in aging. Proc Natl Acad Sci U S A, 2011. 108(25): p. 10237-42.

19. Han, X.J., et al., Regulation of mitochondrial dynamics and neurodegenerative diseases. Acta Med Okayama, 2011. 65(1): p. 1-10.

20. Bugger, $\mathrm{H}$., et al., Uncoupling protein downregulation in doxorubicin-induced heart failure improves mitochondrial coupling but increases reactive oxygen species generation. Cancer Chemother Pharmacol, 2010.

21. Starkov, A.A., et al., Mitochondrial alpha-ketoglutarate dehydrogenase complex generates reactive oxygen species. J Neurosci, 2004. 24(36): p. 7779-88.

22. Dai, D.F., et al., Age-dependent cardiomyopathy in mitochondrial mutator mice is attenuated by overexpression of catalase targeted to mitochondria. Aging Cell, 2010. 9(4): p. 536-44.

23. Kujoth, G.C., et al., Mitochondrial DNA mutations, oxidative stress, and apoptosis in mammalian aging. Science, 2005. 309(5733): p. 481-4. 


\section{Chapter 4}

Transcriptional changes in OXPHOS complex I deficiency are related to anti-oxidant pathways and could explain the disturbed calcium homeostasis.

A.M. Voets, M. Huigsloot, P.J. Lindsey, A.M. Leenders, W.J.H. Koopman, P.H.G.M. Willems, R.J. Rodenburg, J.A.M. Smeitink, H.J.M Smeets

Biochimica et Biophysica Acta (BBA) - Molecular Basis of Disease, In press 


\begin{abstract}
Defective complex I $(\mathrm{Cl})$ is the most common type of oxidative phosphorylation disease, with an incidence of 1 in 5,000 live births. Here, whole genome expression profiling of fibroblasts from $\mathrm{Cl}$ deficient patients was performed to gain insight into the cell pathological mechanism. Our results suggest that patient fibroblasts responded to oxidative stress by Nrf2-mediated induction of the glutathione antioxidant system and Gadd45-mediated activation of the DNA damage response pathway. Furthermore, the observed reduced expression of selenoproteins, might explain the disturbed calcium homeostasis previously described for the patient fibroblasts and might be linked to endoplasmic reticulum stress. These results suggest that both glutathione and selenium metabolism are potentially therapeutic targets in $\mathrm{Cl}$ deficiency.
\end{abstract}

\title{
Key words
}

Gene expression, complex I deficiency, mitochondria, oxidative stress, selenoproteins, Nrf2 


\section{Introduction}

Mitochondria produce most of the cellular ATP through the process of oxidative phosphorylation (OXPHOS). The OXPHOS system is comprised of four multisubunit electron transport chain (ETC) complexes (Complex I-IV) and the $F_{0} / F_{1}$-ATP-synthase (Complex V). Complex I (CI) is the largest of the OXPHOS complexes and one of the entry points of electrons into the ETC. Cl deficiency is the most frequently encountered defect in mitochondrial energy metabolism [1] and is associated with e.g. Leigh disease, Leber hereditary optic neuropathy (LHON), fatal infantile acidosis, neonatal cardiomyopathy with lactic acidosis, leucodystrophy with macrocephaly and hepatopathy with renal tubulopathy $[2,3]$. Although children usually have a normal prenatal development, symptoms start occurring during their first year of life after which the disease deteriorates rapidly and may become fatal [4].

Disease-causing mutations have been described in both the mitochondrial DNA (mtDNA)-encoded (ND1 to ND6, and ND4L genes) and nuclear DNA-encoded structural $\mathrm{Cl}$ subunits (e.g. NDUFS1, NDUFS2, NDUFS4, NDUFS7, NDUFS8) and Cl assembly factors (e.g. B17.2L, NDUFA12L, C20ORF7) [5-26]. The cellular consequences of $\mathrm{Cl}$ deficiency have been extensively studied in patient fibroblasts. $\mathrm{Cl}$ deficiency leads to a slightly depolarized mitochondrial membrane potential [27], increased reactive oxygen species (ROS) levels [28], increased $N A D(P) H$ levels [29], changes in mitochondrial morphology [30] and disturbed calcium homeostasis [31, 32]. However, increased ROS production and mitochondrial fragmentation was not always detectable [33]. A first study investigating transcriptional responses in $\mathrm{Cl}$ deficient fibroblasts cultured with glucose and galactose using a mitochondria-targeted microarray detected the induction of metallothioneins and heat shock proteins and the decreased expression of mtDNA-encoded transcripts [34]. To get an unbiased overview of the underlying pathological processes (not restricted to mitochondria) in $\mathrm{Cl}$ deficiency, we performed whole genome gene expression and additional pathway analysis in fibroblasts of a homogeneous group of $\mathrm{Cl}$ deficient patients with a defect in one of the nuclear encoded structural subunits. The $\mathrm{Cl}$ deficiency might not stress the cell to such an extent that relevant disease-associated gene expression changes can be picked up. Therefore, fibroblasts were deprived of glucose to stimulate energy production through oxidative phosphorylation. In galactose medium, the flow of galactose to glucose-1-phosphate is very slow which obliges cells to obtain ATP from mitochondrial oxidation of pyruvate and glutamine [35].

\section{Material and methods}

Fibroblast cell lines

Fibroblasts were derived from skin biopsies of five patients homozygous or compound heterozygous for nuclear complex I mutations and five controls. The groups were matched for age and sex. All patients have been described previously (\#8807 see [26], \#7898 see [24], \#5175 see [15], \#6613 see [30], \#8382 see [25]). Table 4.1 provides an 
overview of the subjects and physiological parameters in the patient cell lines $[4,28$, $29,32]$. The age- and sex-matched control group consisted of four male subjects, ages 6 months (mo), 1 year (y) 7mo, 1y $6 \mathrm{mo}$, 3y $6 \mathrm{mo}$ respectively and one female subject of $1 \mathrm{y} 11 \mathrm{mo}$.

Fibroblasts were routinely cultured in medium 199 (Gibco, Paisley, UK) supplemented with $10 \%$ fetal calf serum and penicillin/streptomycin (respectively $100 \mathrm{U} / \mathrm{ml}$ and 100 $\mu \mathrm{g} / \mathrm{ml})$. To stimulate energy production by oxidative phosphorylation, fibroblasts were cultured in galactose medium for 48 hours. Galactose medium consisted of DMEM without glucose, without pyruvate and with $4 \mathrm{mM} \mathrm{L-glutamine} \mathrm{(Invitrogen,} \mathrm{Paisley,} \mathrm{UK),}$ $5.5 \mathrm{mM}$ D-galactose (Sigma, Zwijndrecht, Netherlands), $1 \mathrm{mM}$ uridine (Acros, Geel, $\mathrm{BE}$ ), $10 \%$ dialyzed fetal calf serum (Invitrogen) and penicillin/streptomycin, Glucose medium was identical to galactose medium except that D-glucose was present at 5.5 $\mathrm{mM}$ instead of galactose. The stability of the mtDNA was preserved during the course of the experiment in all cell lines under both conditions as no mtDNA deletions or group/treatment specific differences in mtDNA copy number (supplementary table 4.1) could be detected.

\section{Microarray procedure}

Total RNA was isolated using the TRIzol reagent (Invitrogen) and purified with the RNeasy clean-up kit (Qiagen, Hilden, DE). RNA quantity and purity were determined spectrophotometrically using the Nanodrop ND-1000 (Nanodrop Technologies, Wilmington, DE, USA). RNA integrity was assessed by determining the RNA 28S/18S ratio using the Bioanalyser 2100 (Agilent Technologies, Santa Clara, CA, USA). Fibroblast RNA (150 ng) was reverse transcribed into cDNA and amplified in a tworound amplification reaction according to the manufacturer's protocol (Affymetrix, Santa Clara, CA, USA). A mixture of cDNA and added hybridization controls was hybridized on Affymetrix HG-U133 Plus 2.0 chips, followed by staining and washing steps in the GeneChip fluidics station 400 (Affymetrix) according to the manufacturer's procedures. To assess the raw probe signal intensities, chips were scanned using the GeneChip scanner 3000 (Affymetrix).

\section{Microarray data analysis}

Images of the Human Genome U133 Plus 2.0 arrays were quantified with GCOS software (Affymetrix). The microarray data reported in this manuscript have been deposited in NCBI Gene expression omnibus (GEO), accession number GSE27041. The chip description file (CDF) used for the analysis was an update created and freely distributed by the microarray lab of the university of Michigan (http://brainarray.mbni.med.umich.edu; [36]) based on Ensembl (version 10). A more detailed description of this analysis is shown in the supplementary data. Briefly, the genes were analyzed using Gaussian linear regression including the hybridization and labeling spikes, age, sex, passage, mtDNA copy number, and medium. The inference criterion used for comparing the models is their ability to predict the observed data, i.e. models are compared directly through their minimized minus log-likelihood. When the numbers of parameters in models differ, they are penalized by adding the number of estimated parameters, a form of the Akaike information criterion (AIC) [37]. For each 
gene, the group was then added to the model. Then, the group-medium interaction was also added to the model. The gene under consideration was found to be differentially expressed if the AIC of either of these two models decreased compared to the model containing no group effect at all. The genes analyzed and fold changes were loaded into the Pathvisio (version 2.0.8) [38] software package to evaluate the transcripts in relation to known biological processes. The gene database version "Hs_Derby_20090509" was used. Only gene-transcripts with either their average intensities for the control and patient groups above 150 or average intensities for one of these groups above 300 and a 10 percent up or down regulation fold change were used to obtain a ranked list of pathways with differentially expressed genes. Pathvisio software was used to select the pathways with a $Z$ score larger than 1.96 and thus containing relatively high numbers of differentially expressed genes.

\section{Quantitative PCR}

Differentially expressed genes were validated by real-time quantitative PCR (QPCR) with the same RNA samples used for the microarrays. Primers were designed using the NCBI Primer-BLAST tool (NCBI home page; http://www.ncbi.nlm.nih.gov/). cDNA was prepared from $1 \mathrm{\mu g}$ of RNA in a standard reverse transcriptase reaction. PCR was performed in a 7900HT Fast Real-Time PCR System (Applied Biosystems, Foster City, CA, USA) using SensiMixPlus SYBR (Quantace, Finchley, UK). Cycling conditions were: an initial step of 2 minutes at $50^{\circ} \mathrm{C}$, activation of the polymerase at $95^{\circ} \mathrm{C}$ for 10 minutes, and 40 cycles of 15 seconds at $95^{\circ} \mathrm{C}$ followed by 1 minute at $60^{\circ} \mathrm{C}$. The TATAbox binding protein (TBP) gene was used as an internal reference. Genes for which QPCR was performed and the primers used for amplification are listed in supplementary table 4.2. Results were analyzed using Gaussian linear regression, similar to the microarray analysis. The housekeeping gene (TBP), age, sex, passage, mtDNA copy number, RNA integrity number (RIN) and medium were included during the analysis. The AIC was used to assess whether there was a difference between the controls and patients (group effect). All statistical analyses presented were performed using the freely available program R [39] and the publicly available library 'growth' [40].

\section{Western blot analysis}

Protein expression was monitored by Western blot analysis of $12 \%$ SDS-PAGE gels loaded with $40 \mu \mathrm{g}$ of whole cell extract. The following primary antibodies were used: Complex I (NDUFA9; Mitosciences, Oregon, USA), HMOX1 (Abcam, Cambridge, UK), GCLM (Sigma), GSR (Santa Cruz, Heidelberg, DE), and TrxR1 (Santa Cruz). Complex II (70kDa Fp; Mitosciences) was used as a loading control. Secondary antibodies used were polyclonal goat anti-mouse IgG/HRP (Dako, DK) and immunoPure goat anti-rabbit IgG/Peroxidase (Pierce Biotechnology, Rockford, IL, USA). Detection of the signal was performed using ECL Western Blotting Substrate (Thermo Scientific, Amsterdam, NL) following the manufacturer's instructions. 


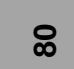

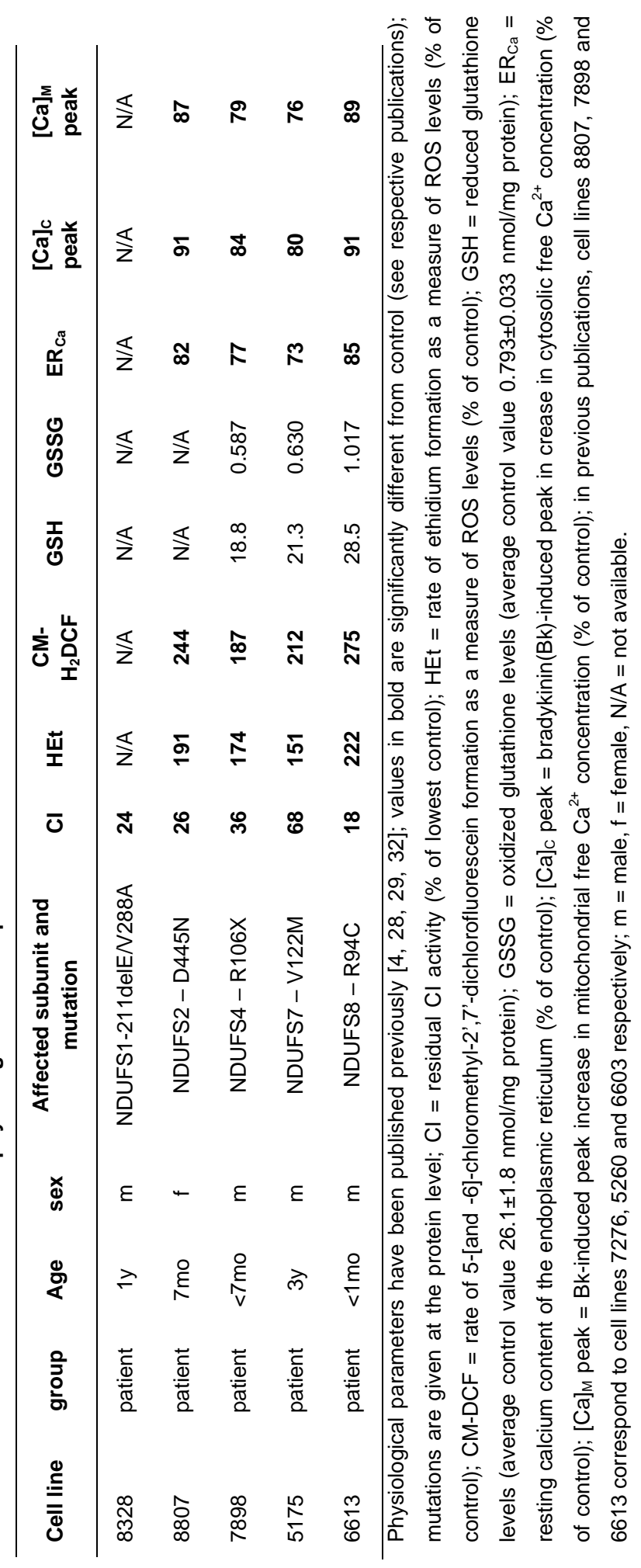




\section{Results}

Global gene expression profiles of fibroblast cell lines from five $\mathrm{Cl}$ deficient patients with a mutation in a nuclear $\mathrm{Cl}$ gene were characterized in glucose and galactose medium to identify processes involved in pathogenesis, novel biomarkers for $\mathrm{Cl}$ deficiency and potential future targets for therapeutic interventions. In order to gain insight into the changes in essential cellular processes rather than single genes, genes were clustered based on cellular function and analyzed by pathway analysis using Pathvisio software [38].

\section{Gene expression analysis}

In total, disease state, culture condition and their interaction significantly altered the expression of 3,279 genes by more than 10 percent. The disease state induced fold changes between 0.08 and 6.89 (of which $79 \%$ between 0.66 and 1.5 (10-50\% change)) in the glucose condition and between 0.08 and 12.18 (69\% between 0.66 and $1.5)$ in the galactose condition. Furthermore, the different culture conditions caused gene expression changes of 0.32 to 2.91 (90\% between 0.66 and 1.5 ) and 0.11 to 29.74 fold (46\% between 0.66 and 1.5 ) in controls and patients respectively. Pathway analysis was performed to identify processes that were altered due to the cumulative effect of differentially expressed genes (table 4.2, supplementary table 4.3). The sum of the differentially expressed genes in a pathway can lead to increased expression, decreased expression or a miscellaneous effect (both inhibition and stimulation), which is indicated for each pathway.

\section{Changes due to culture condition only}

The metabolic switch due to glucose deprivation of the cells in galactose medium changed the expression of 2,888 genes. This included genes with a larger/smaller fold change or an opposite fold change in patients compared with controls. If only genes were included that showed the same effect on galactose compared with glucose for both groups, 1,304 genes were changed and these could be mapped to the pathways cholesterol biosynthesis (increased in galactose), proteasome degradation (miscellaneous effect) and prostaglandin synthesis and regulation (increased in galactose) (figure 4.1B).

\section{Changes due to complex I deficiency}

Between patients and controls, 1,407 and 2,335 genes were significantly changed in glucose and galactose, respectively, with an overlap of 1,199 genes. Pathways that showed a significant change only in the normal glucose condition included the pentose phosphate pathway (increased in patients), heme biosynthesis (decreased in patients), alanine and aspartate metabolism (increased in patients), urea cycle and metabolism of amino groups (increased in patients) and ganglio spingolipid metabolism (decreased in patients). Furthermore, some pathways were significantly altered in both normal glucose and galactose condition: senescence and autophagy (miscellaneous effect), selenium (decreased in patients), mismatch repair (decreased in patients) and nucleotide metabolism (increased in patients) (figure 4.1A). 


\begin{tabular}{|c|c|c|c|c|c|c|c|c|}
\hline Pathway & 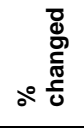 & $\begin{array}{l}0 \\
\overline{0} \\
心 \\
N\end{array}$ & 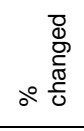 & $\begin{array}{l}0 \\
\stackrel{0}{0} \\
\stackrel{\infty}{N} \\
N\end{array}$ & 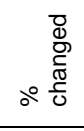 & $\begin{array}{l}0 \\
\stackrel{0}{0} \\
\stackrel{\omega}{0} \\
N\end{array}$ & 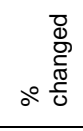 & $\begin{array}{l}0 \\
\bar{O} \\
心 \\
N\end{array}$ \\
\hline \multicolumn{9}{|c|}{ Changes due to culture condition only } \\
\hline Cholesterol Biosynthesis & 14.29 & 0.53 & 14.29 & -0.32 & 57.14 & 5.92 & 0.00 & -1.34 \\
\hline Proteasome Degradation & 13.33 & 0.85 & 25.00 & 1.53 & 18.33 & 2.20 & 15.00 & 0.89 \\
\hline $\begin{array}{l}\text { Prostaglandin Synthesis and } \\
\text { Regulation }\end{array}$ & 13.33 & 0.60 & 20.00 & 0.36 & 23.33 & 2.47 & 6.67 & -0.81 \\
\hline \multicolumn{9}{|c|}{ Changes due to disease, notable in normal condition } \\
\hline Pentose Phosphate Pathway & 57.14 & 4.15 & 42.86 & 1.76 & 28.57 & 1.65 & 14.29 & 0.24 \\
\hline Heme Biosynthesis & 44.44 & 3.43 & 33.33 & 1.25 & 22.22 & 1.24 & 0.00 & -1.08 \\
\hline $\begin{array}{l}\text { Alanine and aspartate } \\
\text { metabolism }\end{array}$ & 33.33 & 2.69 & 33.33 & 1.44 & 25.00 & 1.75 & 8.33 & -0.33 \\
\hline $\begin{array}{l}\text { Urea cycle and metabolism of } \\
\text { amino groups }\end{array}$ & 26.32 & 2.36 & 31.58 & 1.61 & 31.58 & 3.17 & 10.53 & -0.12 \\
\hline Ganglio Sphingolipid Metabolism & 26.67 & 2.14 & 33.33 & 1.61 & 33.33 & 3.04 & 6.67 & -0.57 \\
\hline Adipogenesis & 15.70 & 2.10 & 23.14 & 1.65 & 11.57 & 0.62 & 12.40 & 0.36 \\
\hline \multicolumn{9}{|c|}{ Changes due to disease, notable in normal and selective condition } \\
\hline Senescence and Autophagy & 22.58 & 4.07 & 27.96 & 2.68 & 15.05 & 1.68 & 13.98 & 0.80 \\
\hline Selenium & 17.57 & 2.17 & 29.73 & 2.78 & 9.46 & -0.13 & 17.57 & 1.70 \\
\hline Mismatch repair & 33.33 & 2.32 & 44.44 & 2.12 & 11.11 & 0.12 & 22.22 & 1.03 \\
\hline Nucleotide Metabolism & 27.78 & 2.51 & 38.89 & 2.39 & 22.22 & 1.75 & 22.22 & 1.45 \\
\hline \multicolumn{9}{|c|}{ Changes due to disease that interact with culture condition } \\
\hline Oxidative Stress & 22.22 & 2.11 & 48.15 & 4.20 & 14.81 & 0.85 & 37.04 & 4.22 \\
\hline $\begin{array}{l}\text { Selenium metabolism/ } \\
\text { Selenoproteins }\end{array}$ & 28.13 & 3.41 & 40.63 & 3.45 & 15.63 & 1.08 & 28.13 & 3.00 \\
\hline TGF Beta Signaling Pathway & 19.23 & 2.22 & 34.62 & 3.26 & 11.54 & 0.39 & 26.92 & 3.56 \\
\hline Translation Factors & 21.43 & 2.46 & 26.19 & 1.48 & 19.05 & 1.99 & 21.43 & 2.07 \\
\hline \multicolumn{9}{|c|}{ Changes due to interaction disease-culture condition only } \\
\hline Cell cycle & 14.12 & 1.26 & 32.94 & 3.78 & 10.59 & 0.21 & 24.71 & 3.92 \\
\hline G1 to $S$ cell cycle control & 12.31 & 0.61 & 27.69 & 2.17 & 9.23 & -0.19 & 21.54 & 2.61 \\
\hline $\begin{array}{l}\text { Androgen Receptor Signaling } \\
\text { Pathway }\end{array}$ & 8.41 & -0.57 & 25.23 & 2.12 & 6.54 & -1.19 & 21.50 & 3.35 \\
\hline Glutathione metabolism & 6.25 & -0.51 & 37.50 & 2.10 & 0.00 & -1.33 & 37.50 & 3.30 \\
\hline Keap1-Nrf2 & 7.69 & -0.28 & 46.15 & 2.72 & 15.38 & 0.66 & 38.46 & 3.08 \\
\hline DNA damage response & 11.94 & 0.52 & 31.34 & 3.00 & 11.94 & 0.56 & 23.88 & 3.26 \\
\hline Glycogen Metabolism & 11.43 & 0.27 & 31.43 & 2.17 & 14.29 & 0.87 & 25.71 & 2.69 \\
\hline $\begin{array}{l}\text { TGF-beta Receptor Signaling } \\
\text { Pathway }\end{array}$ & 10.27 & 0.09 & 22.60 & 1.64 & 10.96 & 0.43 & 17.12 & 2.24 \\
\hline Id Signaling Pathway & 6.12 & -0.92 & 22.45 & 0.91 & 10.20 & 0.07 & 20.41 & 2.01 \\
\hline $\begin{array}{l}\text { TNF-alpha/NF-kB Signaling } \\
\text { Pathway }\end{array}$ & 8.00 & -0.93 & 20.00 & 0.88 & 8.57 & -0.61 & 16.00 & 1.98 \\
\hline
\end{tabular}

Every pathway counts at least 7 genes measured by the microarray. A Z score of more than 1.96 indicates that a pathway is significantly changed (shown in bold). 
A

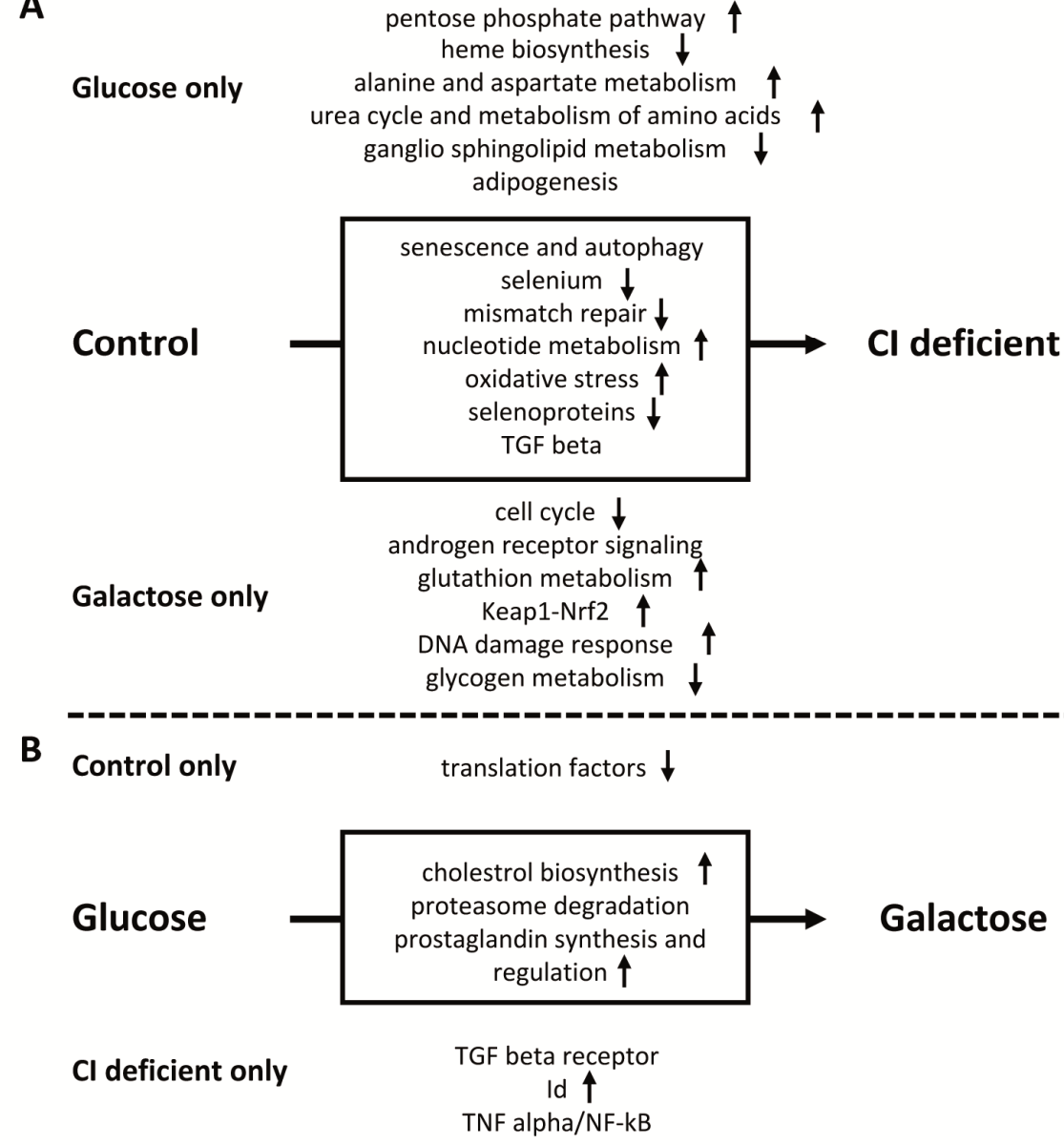

Figure 4.1. Pathways significantly different $(A)$ in controls and patients and $(B)$ in glucose and galactose conditions. Pathways in the box are changed in both culture conditions (A) or groups (B). Pathways above and below the box are only changed in the indicated condition (A) or group (B). Arrows indicate increased or decreased expression; for pathways without arrow, the direction of the change is not clear.

Changes due to disease and interacting with the culture condition

There were 1,584 genes that differed between patients and controls in the glucose and/or galactose culture condition, but for which patients showed a different response to the galactose condition compared with the controls. A different response could be a response to galactose in only the patients or the controls, a quantitative difference or an opposite effect. When considering these genes, part of them were altered as a result of the $\mathrm{Cl}$ deficiency, but additionally showed an interaction between this deficiency and the culture condition. Altered pathways included oxidative stress (increased in patients and further increased in galactose medium), selenium metabolism and selenoproteins (decreased in patients and further decreased in galactose medium), the TGF beta 
signaling pathway (miscellaneous effect) and translation factors (decreased in patients). The remainder of the genes was only altered in patient cells relative to control cells on galactose medium but not in glucose medium. This last group of genes was mapped to pathways involved in cell cycle progression (decreased in patients), oxidative stress (increased in patients; Keap1-NRF2, glutathione metabolism, DNA damage response) and signaling (miscellaneous effect; androgen receptor, TGF beta receptor, Id, TNF alpha/NF-kB). A general overview of all significantly changed pathways is shown in figure 4.1.

\section{QPCR and western blot validation of microarray data}

From the pathway analysis, representative genes with fold changes of more than fifty percent were selected and validated by QPCR (table 4.3). For all except one gene (C$M Y C$ ), the QPCR method detected a difference between patients and controls comparable with the microarray analysis. Furthermore, to correlate gene expression and protein levels, western blot analysis was performed for proteins selected from the genes with relatively high gene expression fold changes in significantly altered pathways for patients versus controls in galactose medium. Significantly increased expression was confirmed for HMOX and GCLC, a similar trend in protein expression was observed for TXNRD1 but no change was detected for GSR (figure 4.2). Additionally, western blot analysis also revealed that complex I deficiency in the cell lines was accompanied by reduced protein levels of complex I (figure 4.2).

\section{Discussion}

During the last decade, studies have generated extensive knowledge on the pathogenesis of $\mathrm{Cl}$ deficiency $[4,28,29,32]$. In spite of these advances in physiological, biochemical and genetic insights, the total picture is far from complete. In the present study, gene expression analysis was performed for a more general overview of the processes involved in $\mathrm{Cl}$ deficiency in fibroblasts. According to recent models of complex I assembly, the selected patients all had mutations in structural $\mathrm{Cl}$ subunits of the dehydrogenase and hydrogenase modules of the hydrophilic peripheral arm of complex I protruding in the matrix. These modules are involved in oxidation of $\mathrm{NADH}$ to $\mathrm{NAD}^{+}$and electron transfer to ubiquinone [41] and mutations in their proteins disturb these fluxes, leading to $\mathrm{Cl}$ deficiency. Therefore, we consider these patients as a group with a common pathophysiological basis, making them suitable for genomewide gene expression approaches.

Differential expression of a number of genes and proteins in relevant pathways was validated and related to available physiological and biochemical data.

Legend Table 4.3. Genes in italics did not show an interaction between disease effect and medium effect with QPCR analysis (in contrast to microarray analysis) but are still able to distinguish patients from controls in both culture media. 
Table 4.3. Selected genes for the QPCR signature

\begin{tabular}{|c|c|c|c|c|c|c|c|c|}
\hline \multirow{3}{*}{ Gene symbol } & \multicolumn{4}{|c|}{ Disease effect } & \multicolumn{4}{|c|}{ Medium effect } \\
\hline & \multicolumn{2}{|c|}{$\begin{array}{l}\text { Glucose } \\
\text { condition }\end{array}$} & \multicolumn{2}{|c|}{$\begin{array}{l}\text { Galactose } \\
\text { condition }\end{array}$} & \multicolumn{2}{|c|}{ Controls } & \multicolumn{2}{|c|}{ Patients } \\
\hline & Array & QPCR & Array & QPCR & Array & QPCR & Array & QPCR \\
\hline \multicolumn{9}{|l|}{ Keap1-Nrf2 } \\
\hline NRF2 & & 0.77 & 1.30 & 1.42 & 0.82 & 0.77 & 1.25 & 1.42 \\
\hline KEAP1 & & 0.77 & 1.28 & 1.27 & & & 1.68 & 1.58 \\
\hline \multicolumn{9}{|c|}{ Oxidative stress } \\
\hline$C Y B A$ & & 0.56 & 0.63 & 0.56 & & 0.62 & 0.63 & 0.62 \\
\hline FOS & 0.44 & 0.48 & 1.55 & 2.10 & 0.62 & & 2.19 & 2.85 \\
\hline HMOX1 & & & 7.23 & 17.58 & & & 9.76 & 28.95 \\
\hline \multicolumn{9}{|c|}{ Glutathione metabolism } \\
\hline GSR & & & 1.96 & 2.46 & & & 2.31 & 3.23 \\
\hline IDH1 & & & 1.50 & 1.79 & & 1.27 & 1.88 & 2.61 \\
\hline GCLC & & & 1.90 & 1.82 & & & 2.38 & 2.66 \\
\hline GCLM & & & 1.94 & 2.67 & & & 1.91 & 2.81 \\
\hline \multicolumn{9}{|c|}{ Selenium metabolism/Selenoproteins } \\
\hline SELK & & & 2.14 & 2.30 & & & 2.01 & 2.36 \\
\hline SEPP1 & 0.38 & 0.33 & 0.21 & 0.33 & 0.59 & 0.39 & 0.32 & 0.39 \\
\hline \multicolumn{9}{|c|}{ Pentose phosphate pathway } \\
\hline PGD & & & 1.75 & 1.55 & & & 2.10 & 2.36 \\
\hline \multicolumn{9}{|c|}{ DNA damage response } \\
\hline GADD45A & & 1.86 & 2.25 & 3.20 & & & 1.89 & 2.08 \\
\hline GADD45B & 1.71 & 3.56 & 3.40 & 3.56 & & 1.81 & 1.84 & 1.81 \\
\hline TNFRSF10B & & & 2.05 & 1.69 & & & 2.12 & 2.11 \\
\hline C-MYC & & & 1.90 & & & 1.78 & 1.75 & 1.78 \\
\hline \multicolumn{9}{|c|}{ Cell cycle - anaphase regulation } \\
\hline ESPL1 & & & 0.60 & 0.35 & 1.69 & 2.04 & & \\
\hline CDC20 & & 0.48 & 0.38 & 0.48 & 2.55 & & & \\
\hline \multicolumn{9}{|c|}{ Nucleotide metabolism } \\
\hline PRPS1 & 1.77 & 1.79 & & 0.59 & & & 0.50 & 0.29 \\
\hline \multicolumn{9}{|c|}{ Glycogen metabolism } \\
\hline GBE1 & 0.87 & & 1.43 & 1.74 & & & 1.63 & 1.70 \\
\hline \multicolumn{9}{|c|}{ Senescence and autophagy } \\
\hline ING1 & & 0.71 & 1.60 & & & & 1.91 & 1.69 \\
\hline \multicolumn{9}{|c|}{ TGF Beta signaling pathway } \\
\hline LTBP1 & 1.70 & & 0.60 & 0.23 & & 1.97 & 0.44 & 0.54 \\
\hline TGIF & & & 1.46 & 1.65 & & & 1.71 & 2.60 \\
\hline \multicolumn{9}{|l|}{ Adipogenesis } \\
\hline RORA & & 1.49 & 1.86 & 2.59 & & & 1.84 & 2.41 \\
\hline DDIT3 & & & 2.61 & 2.50 & & 1.65 & 2.24 & 3.46 \\
\hline LMNA & & & 0.45 & 0.44 & & & 0.45 & 0.46 \\
\hline ADFP & & 0.66 & 1.41 & & & & 1.95 & 1.84 \\
\hline
\end{tabular}


A

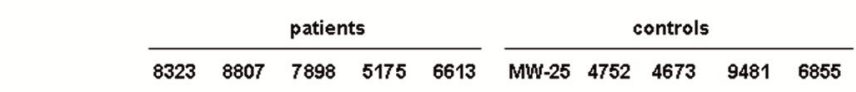

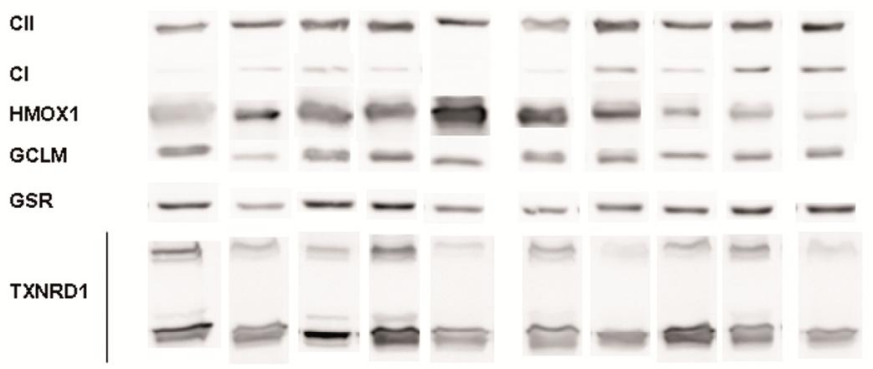

B

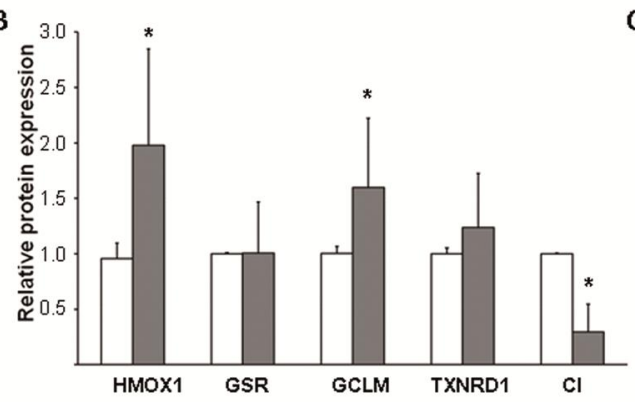

C

\begin{tabular}{|c|ccc|}
\hline Gene & microarray & QPCR & western blot \\
\hline HMOX1 & 7.23 & 17.58 & 1.98 \\
GSR & 1.96 & 2.46 & 1.01 \\
GCLM & 1.94 & 2.67 & 1.6 \\
TXNRD1 & 2.24 & N/A & 1.24 \\
\hline
\end{tabular}

Figure 4.2. Western blot validation of mRNA results. (A) Original western blot results and (B) a graphical representation of differences in protein levels for complex I (Cl), HMOX, GSR, GCLM and TXNRD1 between controls (white bars) and patients (grey bars) fibroblasts cultured with galactose medium, normalized for the expression in controls ${ }^{*} p<0.05$ patients versus controls galactose). Complex II (CII) was used as a reference. In panel C, microarray, QPCR and western blot results for these genes in patients versus controls in galactose medium were compared.

\section{Experimental set-up}

To be able to examine the consequences of $\mathrm{Cl}$ deficiency in fibroblasts (which are mainly glycolytic), cell lines were challenged to use their OXPHOS system for energy production by culturing in galactose medium in the absence of glucose [33,34]. Our analytical model corrected for a number of potential 'noise' factors (biological e.g. age, sex and passage; technical e.g. chip effect), in contrast to a previous study [34]. The approach differed further from this study [34] by type and scope of the array (Affymetrix GeneCHIPS versus home-made two-color cDNA microarrays with selected mitochondria-related genes). The current study provides a well-controlled and more complete picture of the molecular processes in fibroblasts of $\mathrm{Cl}$ patients. These differences can explain why only eleven genes showing differential expression in the previous study could be confirmed [34] (supplementary table 4.4). Unfortunately, nine additional genes could not be analyzed due to the use of the more accurate updated probe set definitions [36] and thirteen genes had low signal intensities, which made comparison impossible. However, the eleven confirmed results included multiple metallothionein transcripts, which were key elements in the previous study [34]. 


\section{General gene expression analysis results}

Linear regression analysis showed significantly altered expression of in total 3,279 genes due to disease, culture medium or a combination of both. As expected, gene expression differences were the highest when comparing patient and control cells cultured in galactose medium and when comparing the glucose and galactose condition in patient fibroblasts. Both the number of differentially expressed genes and the expression difference were higher. In control cells, less than $10 \%$ of the differentially expressed genes showed fold changes of more than $50 \%$ in galactose compared with glucose indicating that control cells were better capable of handling the galactose challenge. The altered expression of a number of genes was validated by QPCR and all of these, except for one gene $(C-M Y C)$, showed a response similar to the microarray thus confirming the overall reliability of the microarray data. Furthermore, for two genes, increased gene expression was accompanied by increased protein expression, validating the biological relevance of the detected differences in gene and pathway expression.

\section{Differentially expressed processes}

To identify processes that were altered due to the cumulative effect of differentially expressed genes in such a process, pathway analysis was performed. Pathways that were only changed in patient versus control fibroblasts both cultured in the presence of glucose were mainly involved in metabolism and included in most cases only a small number of genes with limited fold changes. This suggests that under glycolytic conditions, fibroblasts can adapt relatively easily to alternative energy sources. Therefore, we focused on processes that were changed due to the combination of disease and galactose culturing when the cells are forced to use their oxidative phosphorylation system.

\section{Oxidative stress}

Although the oxidative stress pathway was significantly changed when comparing patient and control cells on glucose, the difference was more significant (higher $Z$ score) and more genes were involved on galactose medium. This is consistent with the observation of increased ROS levels in the CI deficient fibroblasts (table 4.1; [4, 28]). Furthermore, in an independent study with fibroblasts from OXPHOS (including $\mathrm{CI}$ deficient) patients, we observed higher ROS levels when fibroblasts (including controls) were cultured with galactose compared with glucose (manuscript in preparation). Transcripts of genes involved in glutathione homeostasis were increased, but transcription of the other antioxidant enzymes, catalase and superoxide dismutase (mitochondrial and extracellular), was decreased. Although enzyme activity measurements should confirm these results, this suggests that the glutathione antioxidant system is the main defense mechanism counterbalancing increased ROS production in $\mathrm{Cl}$ fibroblasts. Additionally, the induction of the DNA damage response through increased expression of GADD45A and GADD45B could further emphasize cellular stress. Gadd45-mediated growth arrest [42] might explain why fibroblasts of $\mathrm{Cl}$ deficient patients proliferate at a slower rate than healthy control fibroblasts. 
A number of redox-sensitive genes that were significantly changed in this study (HMOX1, CAT and SOD3) were not reported in a previous study [34], most likely due to a different analytical approach. However, the induction of a number of metallothionein genes was detected in both studies (supplementary table 4.4). Metallothioneins are considered potent antioxidants of which the expression is induced by ROS, heavy metals and other forms of cellular stress [34]. Another study with different genetically characterized $\mathrm{Cl}$ deficient patient fibroblast cell lines (NDUFV1 p.W51X + p.T423M and NDUFA1 p.R37S + G8R) did not detect differences in catalase and glutathione reductase protein expression and only one of the three patient cell lines showed the induction of mitochondrial superoxide dismutase protein [33]. This could be explained by the fact that, in contrast to our cell lines (table 4.1; [4, 28]), their cell lines did not show increased ROS production, explaining the lack of increased antioxidant defense. It also suggests that the $\mathrm{Cl}$ mutations of these patients might have different consequences than the ones studied here.

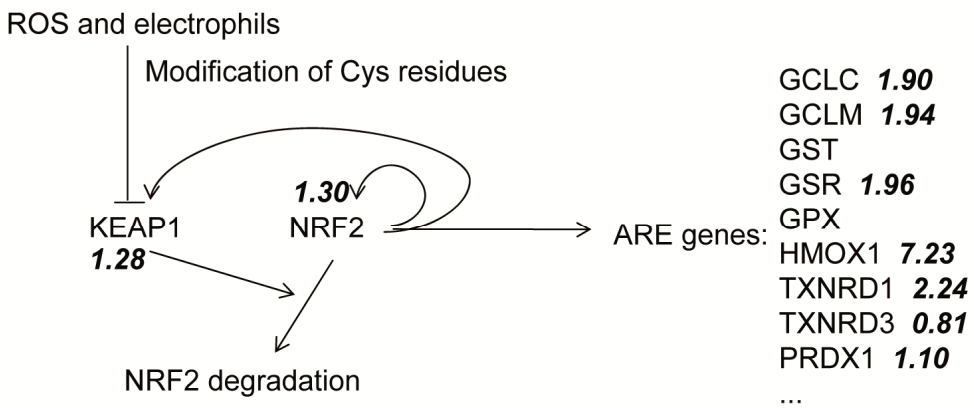

Figure 4.3. Activation of the KEAP1-NRF2 pathway in patient fibroblasts cultured with galactose. Fold changes of the significantly changed genes (patients versus control cultured with galactose) are shown in bold italics.

\section{KEAP1-NRF2-glutathione}

One of the most obvious differentially expressed pathways was the KEAP1-NRF2 pathway, leading to the activation of a large number of genes involved in glutathione homeostasis and the thioredoxin and peroxiredoxin antioxidant defense (figure 4.3). ROS-mediated modification of cysteine (Cys) residues in Keap1 is known to result in diminished binding of Nrf2, leading to decreased Nrf2 degradation, its translocation to the nucleus and transcription of genes containing the antioxidant response element (ARE) in their promoter region [43, 44]. Activation of NRF2 signaling was described previously in fibroblasts of patients with a mutation in the mitochondrial ATP6 gene [45]. Consistent with a lack of NRF2 signaling, glutathione levels in patient fibroblasts were not significantly different from controls in de glucose situation (table 4.1; [29]). However, reduced glutathione (GSH) levels of cell lines 7898 and 5175 were at the lower limit of control values and oxidized glutathione (GSSG) levels were at the higher limit of control values for cell line 6613 [29]. It has been suggested previously that antioxidants present in the culture medium may quench ROS [46], which might make up-regulation of the 
antioxidants unnecessary. With the induction of glutathione homeostasis genes, it can be expected that glutathione levels are increased in the galactose situation to buffer the additional free radicals and prevent oxidative damage. Up-regulation of GCLC was confirmed at the protein level.

\section{Selenium and selenoproteins}

Pathway analysis revealed significant differences in the expression of the selenium (metabolism) and selenoprotein genes in control and patient fibroblasts. Although some overlap exists between these pathways and the oxidative stress pathway, the functions of many of the selenoproteins are unknown [47]. Selenoproteins are a group of proteins that contain selenocystein as an integral part of their polypeptide chain and include different protein families such as the thioredoxin reductases (TXNRDs) and glutathione peroxidases (GPXs), and a number of alphabetically annotated selenoproteins (SelK, SelM, SelN...) [47, 48]. While some classes are definitely involved in antioxidant pathways (GPXs) and redox pathways (TXNRDs), others have been implicated to play a role in calcium homeostasis (SeIN) [47]. Recently, it was shown that association of SelN with the ryanodine receptor was necessary to control release of calcium from intracellular endoplasmic reticulum stores [49]. Thus, a decrease of SEPN1 transcripts, might correlate with the decreased calcium fluxes measured in the studied patient cell lines (table 4.1 ; [31, 32]). If confirmed this might provide a new lead in the pathogenesis of $\mathrm{Cl}$ deficiency. In HepG2 cells, SelK was induced by endoplasmic reticulum stress and protected the cells from stress induced apoptosis [50]. The increased mRNA expression of TXNRD1 and SelK, opposed to the decreased expression of multiple other selenoproteins, might suggest the preferential expression of selenoproteins involved in stress reduction in oxidative $\mathrm{Cl}$ deficient patient fibroblasts. The expression of selenoproteins is depending on the cellular selenium status [51]. Selenium deficiency has been shown to induce the expression of Nrf2 target genes [51, 52], providing a link between the observed induction of the Keap1-Nrf2 pathway and the decreased expression of selenoproteins. Therefore, monitoring and adjusting selenium levels might be beneficial for patients with complex I deficiency. Dietary selenium has been supplemented for the treatment or prevention of cancer [53] and Alzheimer's disease [54]. This study emphasizes the relevance of testing the applicability and efficiency of selenium supplementation also for $\mathrm{Cl}$ deficiency, which should be tested first in vitro and in animal models and later in clinical trials.

\section{Conclusion}

The gene expression analysis supports the changes in oxidative stress levels previously measured in fibroblasts of $\mathrm{Cl}$ deficient patients. It was shown that the cells adapt to and protect themselves against a higher oxidative state through transcriptional regulation of the NRF2 pathway. Additionally, altered expression of selenoproteins provided a new way to explain diminished calcium fluxes in the $\mathrm{Cl}$ deficient fibroblasts. Altogether, the glutathione and selenium pathways might be considered as (new) targets for future therapeutic interventions. Extending our approach to other $\mathrm{Cl}$ deficient 
patient groups with functionally different defects (assembly factor or mtDNA defects affecting the $\mathrm{Cl}$ proton translocation) in different genes will elucidate if the mechanisms identified are common to all complex I patients or if heterogeneity of pathophysiological processes exist .

\section{Acknowledgements}

This work was supported by the Dutch IOP Genomics grant IGE05003. We would like to thank S. Vanherle for technical expertise and help.

\section{Supplementary data}

Supplementary table 4.1. mtDNA copy number results

Supplementary table 4.2. QPCR primers for microarray validation

Supplementary table 4.3. Differentially expressed genes in significantly changed pathways.

Supplementary table 4.4. Differentially expressed genes in the mitochondria-targeted study.

Supplementary material - Microarray statistical analysis

Supplementary material is available online at doi:10.1016/j.bbadis.2011.10.009

\section{References}

1. Smeitink, J., L. van den Heuvel, and S. DiMauro, The genetics and pathology of oxidative phosphorylation. Nat Rev Genet, 2001. 2(5): p. 342-52.

2. Loeffen, J.L., et al., Isolated complex I deficiency in children: clinical, biochemical and genetic aspects. Hum Mutat, 2000. 15(2): p. 123-34.

3. Pitkanen, S., et al., NADH-coenzyme Q reductase (complex I) deficiency: heterogeneity in phenotype and biochemical findings. J Inherit Metab Dis, 1996. 19(5): p. 675-86.

4. Distelmaier, F., et al., Mitochondrial complex I deficiency: from organelle dysfunction to clinical disease. Brain, 2009. 132(Pt 4): p. 833-42.

5. Dunning, C.J., et al., Human CIA30 is involved in the early assembly of mitochondrial complex I and mutations in its gene cause disease. EMBO J, 2007. 26(13): p. 322737.

6. Benit, P., et al., Mutant NDUFV2 subunit of mitochondrial complex I causes early onset hypertrophic cardiomyopathy and encephalopathy. Hum Mutat, 2003. 21(6): p. 582-6.

7. Benit, P., et al., Large-scale deletion and point mutations of the nuclear NDUFV1 and NDUFS1 genes in mitochondrial complex I deficiency. Am J Hum Genet, 2001. 68(6): p. 1344-52.

8. Benit, P., et al., Mutant NDUFS3 subunit of mitochondrial complex I causes Leigh syndrome. J Med Genet, 2004. 41(1): p. 14-7.

9. Berger, I., et al., Mitochondrial complex I deficiency caused by a deleterious NDUFA11 mutation. Ann Neurol, 2008. 63(3): p. 405-8. 
10. Fernandez-Moreira, D., et al., X-linked NDUFA1 gene mutations associated with mitochondrial encephalomyopathy. Ann Neurol, 2007. 61(1): p. 73-83.

11. Hoefs, S.J., et al., NDUFA2 complex I mutation leads to Leigh disease. Am J Hum Genet, 2008. 82(6): p. 1306-15.

12. Kirby, D.M., et al., NDUFS6 mutations are a novel cause of lethal neonatal mitochondrial complex I deficiency. J Clin Invest, 2004. 114(6): p. 837-45.

13. Loeffen, J., et al., The first nuclear-encoded complex I mutation in a patient with Leigh syndrome. Am J Hum Genet, 1998. 63(6): p. 1598-608.

14. Loeffen, J., et al., Mutations in the complex I NDUFS2 gene of patients with cardiomyopathy and encephalomyopathy. Ann Neurol, 2001. 49(2): p. 195-201.

15. Triepels, R.H., et al., Leigh syndrome associated with a mutation in the NDUFS7 (PSST) nuclear encoded subunit of complex I. Ann Neurol, 1999. 45(6): p. 787-90.

16. Schuelke, M., et al., Mutant NDUFV1 subunit of mitochondrial complex I causes leukodystrophy and myoclonic epilepsy. Nat Genet, 1999. 21(3): p. 260-1.

17. van den Heuvel, L., et al., Demonstration of a new pathogenic mutation in human complex I deficiency: a 5-bp duplication in the nuclear gene encoding the 18-kD (AQDQ) subunit. Am J Hum Genet, 1998. 62(2): p. 262-8.

18. Barghuti, F., et al., The unique neuroradiology of complex I deficiency due to NDUFA12L defect. Mol Genet Metab, 2008. 94(1): p. 78-82.

19. Lazarou, M., et al., Assembly of mitochondrial complex I and defects in disease. Biochim Biophys Acta, 2009. 1793(1): p. 78-88.

20. Ogilvie, I., N.G. Kennaway, and E.A. Shoubridge, A molecular chaperone for mitochondrial complex I assembly is mutated in a progressive encephalopathy. $\mathrm{J}$ Clin Invest, 2005. 115(10): p. 2784-92.

21. Pagliarini, D.J., et al., A mitochondrial protein compendium elucidates complex I disease biology. Cell, 2008. 134(1): p. 112-23.

22. Sugiana, C., et al., Mutation of C20orf7 disrupts complex I assembly and causes lethal neonatal mitochondrial disease. Am J Hum Genet, 2008. 83(4): p. 468-78.

23. Gerards, M., et al., Defective complex I assembly due to C20orf7 mutations as a new cause of Leigh syndrome. J Med Genet, 2009. 47(8): p. 507-12.

24. Budde, S.M., et al., Clinical heterogeneity in patients with mutations in the NDUFS4 gene of mitochondrial complex I. J Inherit Metab Dis, 2003. 26(8): p. 813-5.

25. Hoefs, S.J., et al., Novel mutations in the NDUFS1 gene cause low residual activities in human complex I deficiencies. Mol Genet Metab, 2010. 100(3): p. 251-6.

26. Visch, H.J., et al., Decreased agonist-stimulated mitochondrial ATP production caused by a pathological reduction in endoplasmic reticulum calcium content in human complex I deficiency. Biochim Biophys Acta, 2006. 1762(1): p. 115-23.

27. Koopman, W.J., et al., Computer-assisted live cell analysis of mitochondrial membrane potential, morphology and calcium handling. Methods, 2008. 46(4): p. 304-11.

28. Verkaart, S., et al., Superoxide production is inversely related to complex I activity in inherited complex I deficiency. Biochim Biophys Acta, 2007. 1772(3): p. 373-81.

29. Verkaart, S., et al., Mitochondrial and cytosolic thiol redox state are not detectably altered in isolated human NADH:ubiquinone oxidoreductase deficiency. Biochim Biophys Acta, 2007. 1772(9): p. 1041-51.

30. Koopman, W.J., et al., Mitochondrial network complexity and pathological decrease in complex I activity are tightly correlated in isolated human complex I deficiency. Am J Physiol Cell Physiol, 2005. 289(4): p. C881-90.

31. Valsecchi, F., et al., Calcium and ATP handling in human NADH:ubiquinone oxidoreductase deficiency. Biochim Biophys Acta, 2009. 1792(12): p. 1130-7.

32. Willems, P.H., et al., Mitochondrial Ca2+ homeostasis in human NADH:ubiquinone oxidoreductase deficiency. Cell Calcium, 2008. 44(1): p. 123-33.

33. Moran, M., et al., Mitochondrial bioenergetics and dynamics interplay in complex Ideficient fibroblasts. Biochim Biophys Acta, 2010. 1802(5): p. 443-53. 
34. van der Westhuizen, F.H., et al., Human mitochondrial complex I deficiency: investigating transcriptional responses by microarray. Neuropediatrics, 2003. 34(1): p. 14-22.

35. Reitzer, L.J., B.M. Wice, and D. Kennell, Evidence that glutamine, not sugar, is the major energy source for cultured HeLa cells. J Biol Chem, 1979. 254(8): p. 2669-76.

36. Dai, M., et al., Evolving gene/transcript definitions significantly alter the interpretation of GeneChip data. Nucleic Acids Res, 2005. 33(20): p. e175.

37. Akaike, $\mathrm{H}$. Information theory and an extension of the maximum likelihood principle. in Second International Symposium on Inference Theory. 1973. Budapest: Akadémiai Kiadó.

38. van lersel, M.P., et al., Presenting and exploring biological pathways with PathVisio. BMC Bioinformatics, 2008. 9: p. 399.

39. Ihaka, R. and R. Gentleman, "R: a language for data analysis and graphics". Journal of Computational Graphics and Statistics, 1996. 5(3): p. 299-314.

40. Lindsey, J., Models for repeated measurements, 2nd edition. 1999, Oxford: Oxford University Press. 536.

41. Fernandez-Vizarra, E., V. Tiranti, and M. Zeviani, Assembly of the oxidative phosphorylation system in humans: what we have learned by studying its defects. Biochim Biophys Acta, 2009. 1793(1): p. 200-11.

42. Gao, M., et al., Diverse roles of GADD45alpha in stress signaling. Curr Protein Pept Sci, 2009. 10(4): p. 388-94.

43. Nguyen, T., P. Nioi, and C.B. Pickett, The Nrf2-antioxidant response element signaling pathway and its activation by oxidative stress. J Biol Chem, 2009. 284(20): p. 13291-5.

44. Singh, S., et al., Nrf2-ARE stress response mechanism: A control point in oxidative stress-mediated dysfunctions and chronic inflammatory diseases. Free Radic Res, 2010.

45. Dassa, E.P., et al., The mtDNA NARP mutation activates the actin-Nrf2 signaling of antioxidant defenses. Biochem Biophys Res Commun, 2008. 368(3): p. 620-4.

46. von Kleist-Retzow, J.C., et al., Impaired mitochondrial Ca2+ homeostasis in respiratory chain-deficient cells but efficient compensation of energetic disadvantage by enhanced anaerobic glycolysis due to low ATP steady state levels. Exp Cell Res, 2007. 313(14): p. 3076-89.

47. Bellinger, F.P., et al., Regulation and function of selenoproteins in human disease. Biochem J, 2009. 422(1): p. 11-22.

48. Papp, L.V., et al., From selenium to selenoproteins: synthesis, identity, and their role in human health. Antioxid Redox Signal, 2007. 9(7): p. 775-806.

49. Jurynec, M.J., et al., Selenoprotein $N$ is required for ryanodine receptor calcium release channel activity in human and zebrafish muscle. Proc Natl Acad Sci U S A, 2008. 105(34): p. 12485-90.

50. Du, S., et al., SelK is a novel ER stress-regulated protein and protects HepG2 cells from ER stress agent-induced apoptosis. Arch Biochem Biophys, 2010. 502(2): p. 137-43.

51. Muller, M., et al., Nrf2 target genes are induced under marginal selenium-deficiency. Genes Nutr, 2010. 5(4): p. 297-307.

52. Burk, R.F., et al., Selenium deficiency activates mouse liver Nrf2-ARE but vitamin E deficiency does not. Free Radic Biol Med, 2008. 44(8): p. 1617-23.

53. Klein, E.A., et al., SELECT: the selenium and vitamin E cancer prevention trial. Urol Oncol, 2003. 21(1): p. 59-65.

54. Kryscio, R.J., et al., Designing a large prevention trial: statistical issues. Stat Med, 2004. 23(2): p. 285-96. 


\section{Chapter 5}

Patient-derived fibroblasts indicate oxidative stress status and may justify antioxidant therapy in OXPHOS disorders.

A.M. Voets, P.J. Lindsey, S.J. Vanherle, E.D. Timmer, J.J. Esseling, W.J.H. Koopman, P.H.G.M. Willems, G.C. Schoonderwoerd, D. De Groote, B.T. Poll-The, I.F.M. de Coo, H.J.M. Smeets

Submitted 


\begin{abstract}
Oxidative phosphorylation (OXPHOS) disorders are often associated with increased oxidative stress and antioxidant therapy is frequently given as treatment. However, the role of oxidative stress in OXPHOS disorders or patients is far from clear and consequently the preventive or therapeutic effect of antioxidants is highly anecdotic. Therefore, we performed a systematic study of a panel of oxidative stress parameters (ROS levels, damage and defense) in fibroblasts of twelve well-characterized OXPHOS patients with a defect in the POLG1 gene, in the mitochondrial DNA ( $m$ tDNA)-encoded tRNA-Leu gene $(3243 A>G$ or $3302 A>G)$ and in one of the mtDNAencoded NADH dehydrogenase complex I (Cl) subunits. All except two cell lines (one POLG1 and one tRNA-Leu) showed increased ROS levels compared with controls, but only four (two $\mathrm{Cl}$ and two tRNA-Leu) cell lines provided evidence for increased oxidative protein damage. The absence of a correlation between ROS levels and oxidative protein damage implies differences in damage prevention or correction. This was investigated by gene expression studies, which showed adaptive and compensating changes involving antioxidants and the unfolded protein response, especially in the POLG1 group. This study indicated that patient fibroblasts enable the identification of patients that potentially benefit from antioxidant therapy. Furthermore, the fibroblast model can also be used to search for and test novel, more specific antioxidants or explore ways to stimulate compensatory mechanisms.
\end{abstract}

\title{
Key words
}

oxidative stress, POLG1, Cl deficiency, MELAS, ROS levels, protein carbonyls, glutathione 


\section{Introduction}

Oxidative phosphorylation (OXPHOS) disorders are the most common group of inherited metabolic disorders characterized by a primary dysfunction of the OXPHOS system. The clinical and biochemical heterogeneity of OXPHOS disorders is partly due to the dual (mitochondrial and nuclear DNA) genetic control of mitochondrial energy production. Other factors that could explain this heterogeneity are only partly resolved, awaiting further insight in the pathophysiological processes. Recently, a number of papers have addressed the issue of oxidative stress in OXPHOS disorders (e.g. [1-8]). The electron transport chain in the mitochondria is considered the major source of reactive oxygen species (ROS), which are by-products of the redox reactions necessary to reduce NADH to $\mathrm{NAD}^{+}$[9]. At low levels, ROS behave as signaling molecules [10], but increased levels are damaging for DNA, proteins and lipids as well as detrimental for cellular function [11-14]. Therefore, cells are well equipped with antioxidant systems to control ROS levels. Oxidative stress occurs when the balance between pro-oxidants (ROS) and antioxidants is disturbed and antioxidants are no longer able to maintain normal physiological ROS levels.

Increased ROS production has been described in patients with clinically, biochemically $[4,15,16]$ and/or genetically [17-19] diagnosed OXPHOS disorders, in cybrid models [6-8] and in cell lines where the respiratory chain was inhibited by chemicals [20]. The antioxidant status of tissues or cell cultures from OXPHOS patients [1, 5, 6, 16, 19, 21] was investigated with variable and sometimes contradictory results. Only few studies report the total picture of oxidative stress in these patients, including ROS production, antioxidant defense and the eventual oxidative damage $[6,19,22]$. The goal of this study was to investigate the role of oxidative stress in three different genetically characterized OXPHOS disorders: patients with mutations in the POLG1 gene, patients with a tRNA leucine (tRNA-Leu) mutation in the mtDNA (3243A>G and 3302A>G) causing mitochondrial encephalomyopathy, lactic acidosis and stroke-like episodes (MELAS) and patients with a mutation in one of the mtDNA encoded complex I subunits. Complex I deficiency and tRNA-Leu mutations have been associated with increased oxidative stress previously [19, 23], although not all studies could confirm this [24]. Importantly, different parameters have been characterized to determine oxidative stress, including increased probe oxidation [23] combined with antioxidant expression [24] and increased oxidative damage, or combinations of the three [19]. For POLG1 mutations, a couple of studies were performed in transgenic mice, but again with conflicting conclusions: transgenic mouse models carrying the D257A mutation in the exonuclease domain of pol $y$ did not increase oxidative stress $[25,26]$ whereas transgenic mice with cardiac-targeted human mutant $\mathrm{Y} 955 \mathrm{C}$ pol $\mathrm{y}$, affecting the polymerase domain, did [27].

Our study aimed at investigating parameters including ROS levels, ROS detoxification (glutathione levels, antioxidant gene expression) and oxidative damage (protein carbonyls) in patient-derived fibroblasts as a model system. Because the genetic defect might not stress the cells to such an extent that relevant disease-associated changes 
can be picked up, fibroblasts were deprived of glucose to stimulate energy production through oxidative phosphorylation. Nevertheless, our main focus is on changes due to the genetic defect and not the difference between the two culture conditions. This model will help to resolve the underlying adaptive mechanisms, identify the subgroup of patients that will most likely benefit from antioxidant treatments and test the efficacy of new targeted candidate antioxidants.

\section{Materials and methods}

\section{Patient fibroblast cell lines}

Fibroblasts were derived from skin biopsies of five patients with a POLG1 mutation, four with a tRNA-Leu (m.3243A $>$ G or m.3302A $>$ G) MELAS mutation, three with a mutation in an mtDNA encoded complex I subunit, and three controls (table 5.1). Fibroblasts were routinely cultured in Dulbecco's modified Eagle medium (DMEM; Gibco, Paisley, UK) supplemented with 10\% fetal bovine serum, $0.2 \mathrm{mM}$ uridine (Acros, Geel, BE), penicillin and streptomycin. To stimulate energy production by oxidative phosphorylation, fibroblasts were cultured for 72 hours without glucose in de presence of galactose. Galactose medium consisted of DMEM without glucose supplemented with 5.5mM galactose (Sigma, Zwijndrecht, Netherlands), 20\% FBS, $0.2 \mathrm{mM}$ uridine, penicillin and streptomycin [28]. Measurements were performed in primary cell cultures between passage 7 and 20. Except for the quantification of reactive oxygen species, cultures from the same cell line and condition were pooled and the cell pellet was divided for the different assays.

\section{Quantification of reactive oxygen species}

Reactive oxygen species (ROS) levels were measured as described previously [23]. In short, fibroblasts were incubated in HEPES-Tris medium (132 mM NaCl, $4.2 \mathrm{mM} \mathrm{KCl}, 1$ $\mathrm{mM} \mathrm{CaCl}_{2}, 1 \mathrm{mM} \mathrm{MgCl}$, $10 \mathrm{mM} \mathrm{HEPES}$ and $5.5 \mathrm{mM}$ D-glucose or galactose, $\mathrm{pH}$ 7.4), containing $10 \mu \mathrm{M}$ hydroethidine (HEt; Molecular Probes, Paisley, UK) for 10 minutes at $37^{\circ} \mathrm{C}$. The reaction was stopped by thorough washing of the cells with PBS. Culture dishes were mounted in an incubation chamber placed on the stage of an inverted microscope (Axiovert 200M, Carl Zeiss, Jena, DE) equiped with a Zeiss 40x/1.3 NA fluor objective. The cells were excited at $490 \mathrm{~nm}$ using a monochromator (Polychrome IV, TILL Photonics, Gräfelfing, DE). Fluorescence emission light was directed by a 525DRLP dichroic mirror filter (Omega) onto a CoolSNAP HQ monochrome CCDcamera (Roper Scientific, Vianen, NL) with an acquisition time of $100 \mathrm{~ms}$. Hardware was controlled with Metafluor 6.0 software (Universal Imaging Corporation, Downingtown, PA, USA). Processing and analysis of fluorescence images was performed with MetaMorph 6.1 (Universal Imaging Corporation). All cell lines were examined on at least two but possibly up to four different days. In total, at least 50 cells (range 52-407) were analyzed on each day for each cell line and condition, resulting in a total of at least 150 cells (range 156-568) analyzed for each cell line and condition. 
Table 5.1. Patient characteristics for primary fibroblast cell lines.

\begin{tabular}{|c|c|c|c|c|c|}
\hline Cell line & Sex & $\begin{array}{l}\text { Age at } \\
\text { biopsy } \\
\text { (years) }\end{array}$ & Group $^{a}$ & Mutation(s) ${ }^{b}$ & OXPHOS complex activity $^{c}$ \\
\hline $2862 S$ & $\mathrm{~F}$ & 24 & POLG & p.467A > T HOM & $\begin{array}{c}\text { CI } 67, \text { CII 125, CIII 108, CIV } \\
87, \text { CS } 93\end{array}$ \\
\hline 06E0703 & $\mathrm{F}$ & 16 & POLG & p.467A > T HOM & $\begin{array}{c}\text { CI 54, CII 141, CIII 79, CIV 84, } \\
\text { CS } 71\end{array}$ \\
\hline 00E0741 & $\mathrm{F}$ & $<1$ & POLG & $\begin{array}{l}\text { p. } 227 \mathrm{R}>\mathrm{P}+ \\
\text { p. } 467 \mathrm{~A}>\mathrm{T}\end{array}$ & $\begin{array}{c}\text { Cl 34, CII 76,CIII 31, CIV 50, } \\
\text { CS } 227\end{array}$ \\
\hline 3591 & M & 8 & POLG & $\begin{array}{l}\text { p. } 305 \mathrm{~S}>\mathrm{R}+ \\
\text { p.467A }>T\end{array}$ & $\begin{array}{c}\text { CI 50, CII 86, CIII 57, CIV 89, } \\
\text { CS } 142\end{array}$ \\
\hline $05 E 0536$ & $\mathrm{~F}$ & 1 & POLG & $\begin{array}{l}\text { p. } 467 \mathrm{~A}>\mathrm{T}+ \\
\text { p.957A }>\mathrm{P}\end{array}$ & CI 63, CII 81, CIV 57, CS 150 \\
\hline 2400 & M & 27 & tRNA-Leu & m.3243A>G (80\%) & $\begin{array}{c}\mathrm{Cl} 43, \mathrm{Cll} 86, \mathrm{CIII} 67, \mathrm{CIV} 80, \\
\text { CS } 125\end{array}$ \\
\hline 1933 & M & 27 & tRNA-Leu & m.3243A>G (90\%) & $\begin{array}{c}\text { CI 50, CII 84, CIII 60, CIV 79, } \\
\text { CS } 117\end{array}$ \\
\hline 2830 & $\mathrm{~F}$ & 19 & tRNA-Leu & m.3243A>G (83\%) & $\begin{array}{c}\mathrm{CI} 57, \mathrm{CII} 86, \mathrm{CIII} 77, \mathrm{CIV} 60, \\
\text { CS } 100\end{array}$ \\
\hline 1330 & M & 34 & tRNA-Leu & m.3302A>G (50\%) & $\begin{array}{c}\text { CI 62, CII 100, CIII 79, CIV 83, } \\
\text { CS } 108\end{array}$ \\
\hline 3765 & M & 5 & $\mathrm{Cl}$ & $\begin{array}{c}\text { ND1, } m .3890 G>A \\
(80 \%)\end{array}$ & $\begin{array}{c}\text { CI 34, Cll 69, CIII 72, CIV 114, } \\
\text { CS } 160\end{array}$ \\
\hline 2181 & M & 3 & $\mathrm{Cl}$ & $\begin{array}{c}\text { ND5, } m .13042 G>A \\
(86 \%)\end{array}$ & $\begin{array}{c}\text { CI 50, CII 90, CIII 59, CIV 70, } \\
\text { CS } 110\end{array}$ \\
\hline 1682 & $\mathrm{~F}$ & 4 & $\mathrm{Cl}$ & $\begin{array}{c}\text { ND5, } m .13511 \mathrm{~A}>\mathrm{T} \\
(60 \%)\end{array}$ & $\begin{array}{c}\text { CI 52, CII 94, CIII 91, CIV 115, } \\
\text { CS } 105\end{array}$ \\
\hline C0388 & & & Control & - & n.a. \\
\hline C0407 & & & Control & - & n.a. \\
\hline C2244 & & & Control & - & n.a. \\
\hline
\end{tabular}

\footnotetext{
${ }^{a}$ Patient cell lines are grouped according to their genetic defect: polymerase gamma mutations (POLG), mtDNA tRNA leucine mutation with MELAS phenotype (MELAS) and mtDNA complex I subunit mutation $(\mathrm{Cl}) .{ }^{\mathrm{b}}$ Mutations are given at the protein level for POLG and at the mtDNA level for MELAS and $\mathrm{Cl}$, the mutation percentage of the mtDNA mutations is given between ( ). ${ }^{\mathrm{C}}$ The activities of the different OXPHOS complexes is expressed as the percentage of a control population, normalized to citrate synthase (CS) activity. $\mathrm{Cl}=$ complex I, CII=complex II, CIII=complex III, CIV=complex IV. n.a. = not applicable.
} 
Preparation of lysates for glutathione measurements and protein carbonyls

Cell pellets were resuspended in ice-cold extraction buffer $(0.1 \%$ Triton X-100 and $0.6 \%$ sulfosalicylic acid in $0.1 \mathrm{M}$ potassium phosphate buffer with $5 \mathrm{mM}$ EDTA disodium salt, $\mathrm{pH}$ 7.5) and homogenized with a Teflon pestle. After sonication and 2 freeze-thaw cycles, the suspension was centrifuged for $4 \mathrm{~min}$ at $3000 \mathrm{~g}$ (4 degrees) and the supernatant was stored at -70 degrees until further use. Protein concentrations were determined using the Bio-Rad Protein Assay.

\section{Glutathione measurement}

Total glutathione (reduced (GSH) + oxidized (GSSG)) was measured in the cell lysates as described in [29]. In short, freshly prepared 5,5'-dithio-bis(2-nitrobenzoic acid) (Sigma) and glutathione reductase (Sigma) solutions were mixed and added to the cell lysates $(40 \mu \mathrm{g}$ protein in 20 microliter). After 30s, NADPH (Sigma) was added and the rate of change in absorbance at $412 \mathrm{~nm}$ was proportional to the concentration of total $\mathrm{GSH}$ ([GSH] $+2 \times$ [GSSG]) in the sample. The concentration of total GSH was deduced from the regression curve generated from several standards of GSH. Data were analyzed using the univariate analysis of variance in the SPSS software package.

\section{Protein carbonyl detection}

To detect oxidative modifications of proteins, the Oxyblot ${ }^{\mathrm{TM}}$ Protein Oxidation Detection kit (Millipore, Amsterdam, NL) was used. After derivatization of the carbonyl groups in the cell lysates with di-nitrophenylhydrazone, oxidatively modified proteins were detected by immunoblotting according to the manufacturer's protocol in two replicates. Results were normalized to beta-actin protein levels in different lanes of the same gels.

\section{Oxidative stress related gene expression}

The expression levels of genes involved in oxidative stress and inflammation were measured using the OxyGenes ${ }^{T M}$ microarrays (Probiox SA, Liege, BE) [30]. RNA was isolated from cell pellets with the High Pure RNA Isolation kit (Roche, Woerden, NL). RNA quantity and purity were determined spectrophotometrically using the Nanodrop ND-1000 (Nanodrop Technologies, Wilmington, DE, USA) and RNA integrity was assessed by determining the RNA 28S/18S ratio using the Bioanalyser 2100 (Agilent Technologies, Santa Clara, CA, USA). $2 \mu \mathrm{g}$ of RNA was reverse transcribed into cDNA according to the 3DNA Array 900 protocol (Genisphere, Hatfield, PA, USA). Next, cDNA was hybridized on the OxyGenes ${ }^{T M}$ slides overnight, followed by washing steps and hybridization of $\mathrm{Cy}^{\mathrm{TM}}$ 3-labeled $3 \mathrm{DNA}$ capture reagent according to the manufacturer's procedures (Genisphere). To assess the raw probe signal intensities, slides were scanned using the LS Reloaded laser scanner (Tecan, Männedorf, CH) with gain settings 160, 180 and 200 and analyzed using Array-Pro analyzer software (MediaCybernetics, Bethesda, USA) or scanned using the Agilent High-Resolution Microarray Scanner (Agilent Technologies, Amstelveen, NL) and analyzed using Feature Extraction 10.7 software (Agilent Technologies). 


\section{Data analysis}

The data were analyzed using multivariate Gaussian linear regression including a one (protein carbonyls and Oxygenes ${ }^{\mathrm{TM}}$ ) or two level (ROS levels) random effect to take into account the dependence among observations from the same subject (all assays) and the dependence among the different measurement days (ROS levels). For the ROS level analysis, the medium (glucose and galactose) and the group (control, complex I, tRNA-Leu and POLG) were included during the analysis, as well as interactions among these when required. For the protein carbonyl analysis, the reference protein (beta-actin), concentration, day, gel number, medium and group were included. The inference criterion used for comparing the models reflects their ability to predict the observed data, i.e. models are compared directly through their minimized minus log-likelihood. When the numbers of parameters in models differ, they are penalized by adding the number of estimated parameters, a form of the Akaike information criterion (AIC) [31]. First, all cell lines were analyzed together and then each one was also considered separately. In each case, the relevant group differences were reported if the model with the smallest AIC contained any combination of one or more groups.

For the Oxygenes ${ }^{\mathrm{TM}}$ analysis, one of the 30 housekeeping genes, the pooled sample, the background intensity, the scanner used, the medium and group were included during the analysis as well as interactions among these when required. Similarly to the ROS levels and protein carbonyl analysis, the AIC was used to assess whether there was a group effect. First, each gene was analyzed for all cell lines in the patient groups together and then the analysis was also repeated for all genes but considering each cell line separately. In each analysis, the relevant group differences were reported if the model with the smallest AIC contained any combination of one or more groups.

Importantly, the reported fold changes are estimated values by the models, taking into account both the effect of the mutation and the medium.

\section{Results}

Increased ROS levels in most of the patient cell lines

ROS levels, quantified by the oxidation rate of hydroethidine $(\mathrm{HEt})$, were assessed in the control and patient cell lines under glucose and galactose conditions (table 5.2). Unlike the estimated fold changes for the patient group differences, analysis of individual cell lines and controls did not always estimate a medium effect, i.e. a different fold change for the glucose and galactose condition, due to low power. In these cases, the estimated fold changes represent the difference between the specific patient and control fibroblasts regardless of the medium.

Except for two cell lines, one POLG1 mutant (06E0703; p.467A $>$ T homozygote) and one tRNA-Leu mutant (1330; m.3302A>G), all other cell lines showed increased HEt oxidation compared with control cell lines in glucose and galactose medium. HEt oxidation was highest in the $\mathrm{Cl}$ group, although one of the POLG1 cell lines (05E0536; p.467A $>T+$ p.957A $>P$ ) presented with the highest HEt oxidation rate. Although the main focus was on changes due to the mutation and not the differences between 
culture conditions, six cell lines showed a larger fold change for HEt oxidation (versus controls) in galactose compared with glucose. Galactose medium also induced a 1.53 fold increase in HEt oxidation in controls (table 5.2).

Table 5.2. ROS levels as measured by HEt oxidation.

\begin{tabular}{|c|c|c|}
\hline Cell line & Glucose mean (C.I.) & Galactose mean (C.I.) \\
\hline Average control & 1.00 & $1.53(1.49-1.57)$ \\
\hline \multicolumn{3}{|l|}{ (3 cell lines) } \\
\hline Average POLG & $1.47(1.12-1.82)$ & $1.71(1.34-2.18)$ \\
\hline $2862 S$ & $1.36(1.14-1.63)$ & $1.96(1.65-2.34)$ \\
\hline 06E0703 & \multicolumn{2}{|c|}{$1.12(0.96-1.30)$} \\
\hline 00E0741 & \multicolumn{2}{|c|}{$1.18(1.04-1.33)$} \\
\hline 3591 & \multicolumn{2}{|c|}{$1.51(1.35-1.70)$} \\
\hline 05E0536 & $2.51(2.13-2.95)$ & $4.80(4.08-5.65)$ \\
\hline Average tRNA-Leu & $1.48(1.14-1.83)$ & $1.72(1.35-2.19)$ \\
\hline 2400 & \multicolumn{2}{|c|}{$1.45(1.28-1.64)$} \\
\hline 1933 & $1.30(1.12-1.51)$ & $1.92(1.65-2.23)$ \\
\hline 2830 & $1.74(1.46-2.08)$ & $2.52(2.10-3.03)$ \\
\hline 1330 & \multicolumn{2}{|c|}{$1.10(0.96-1.25)$} \\
\hline Average $\mathrm{Cl}$ & $1.87(1.47-2.27)$ & $2.28(1.73-3.01)$ \\
\hline 3765 & $1.61(1.37-1.90)$ & $2.38(2.01-2.83)$ \\
\hline 2181 & \multicolumn{2}{|c|}{$2.31(1.98-2.69)$} \\
\hline 1682 & \multicolumn{2}{|c|}{$1.36(1.20-1.54)$} \\
\hline
\end{tabular}

HEt oxidation expressed as fold change compared with control in the respective culture medium. Values are estimated fold changes for patients versus controls provided by the model, taking into account both the effect of the mutation and the medium. Values for the patient groups and the individual cell lines were estimated in separate analyses. If the fold change was not significantly different for a cell line between both media, the fold change of the best fitting model was estimated for glucose and galactose together. Values in bold differ significantly from control in the respective medium or glucose and galactose together. ${ }^{\#}$ versus control glucose, only applies for the patient groups comparison.

\section{Oxidative damage}

The levels of protein carbonyls, an index for oxidative protein damage, were significantly elevated in three patient cell lines cultured with glucose and galactose: tRNA-Leu cell lines $2400(80 \%$ m.3243A>G) and $1933(90 \%$ m.3243A>G) and Cl cell 
line $3765(80 \%$ m.3890G>A). Cl cell line $1682(60 \%$ m.13511A $>$ T) only showed increased levels of protein carbonyls compared with controls when cultured with galactose medium (table 5.3).

Table 5.3. Oxidative protein damage (protein carbonyls).

\begin{tabular}{|c|c|}
\hline Cell line & Galactose mean (C.I.) \\
\hline Average control & 1.00 \\
\hline \multicolumn{2}{|l|}{ (3 cell lines) } \\
\hline Average POLG & $1.07(0.94-1.21)$ \\
\hline $2862 S$ & $1.07(0.76-1.50)$ \\
\hline 06E0703 & $0.93(0.81-1.08)$ \\
\hline 00E0741 & $1.14(0.96-1.35)$ \\
\hline 3591 & $1.15(0.92-1.43)$ \\
\hline $05 E 0536$ & $0.92(0.73-1.66)$ \\
\hline Average tRNA-Leu & $1.30(1.13-1.48)$ \\
\hline 2400 & $1.25(1.06-1.47)$ \\
\hline 1933 & $1.56(1.29-1.89)$ \\
\hline 2830 & $1.26(0.90-1.77)$ \\
\hline 1330 & $1.14(0.94-1.39)$ \\
\hline Average $\mathrm{Cl}$ & $1.22(1.06-1.41)$ \\
\hline 3765 & $1.36(1.07-1.74)$ \\
\hline 2181 & $1.08(0.96-1.22)$ \\
\hline 1682 & $1.12(0.76-1.65) \quad 2.22(1.79-2.75)$ \\
\hline
\end{tabular}

Protein carbonyls as measured using the Oxyblot assay, expressed as fold change compared with control in the respective culture medium. Values are estimated fold changes for patients versus controls provided by the model, taking into account both the effect of the mutation and the medium. Values for the patient groups and the individual cell lines were estimated in separate analyses. If the fold change was not significantly different for a cell line or group between both media (due to less power compared with the analysis of the groups), the fold change of the best fitting model was estimated for glucose and galactose together. Values in bold differ significantly from control.

\section{Stress-related gene expression and antioxidant defense}

To examine ROS-related gene expression changes, the transcription levels of stressrelated genes were determined using OxyGenes ${ }^{\mathrm{TM}}$ microarrays. Of the 165 genes on the microarray, 38 genes showed more than $20 \%$ difference in expression above the background level in at least one of the groups and one of the culture media. Of these 38 genes, 10 were altered due to the genetic defect only, as their expression was not influenced by the culture medium in controls and patients (table 5.4). Therefore these were considered specific for the genetic defects and included, amongst others, heat shock protein (HSPA1A), oxidative stress related (SOD1, PON2, MT1M) and inflammatory (ICAM1, IL2RG, TNFRSF1B) genes. The expression of the remaining 28 genes was altered due to both the culture medium and genetic defect (supplementary table 5.1). The POLG group showed the highest number of differentially expressed genes compared with controls, both in glucose and galactose medium. The galactose 
medium induced larger expression changes of 17 stress-related genes in the POLG group (supplementary table 5.1). In contrast, the tRNA-Leu and Cl groups showed less differentially expressed genes, both in glucose and galactose (supplementary table 5.1). A closer examination of the gene expression changes of classical antioxidant genes in the individual cell lines (table 5.5) revealed an inconsistent picture. On the one hand, genes could behave comparable in multiple cell lines of one or more patient groups in one or both culture conditions (e.g. GSR in POLG glucose and galactose, GPX1 in tRNA-Leu galactose, SOD1 in tRNA-Leu and Cl galactose). Whereas, on the other hand, their expression changed differently, either up or down, in cell lines of the same group in one or both culture conditions (e.g. GPX1 in POLG glucose and galactose, SOD1 in tRNA-Leu and $\mathrm{Cl}$ glucose) (table 5.5).

Table 5.4. Stress-related gene expression changes due to disease without medium effect.

\begin{tabular}{lcccc}
\hline Gene & $\begin{array}{c}\text { FC Cl } \\
\text { vs control }\end{array}$ & $\begin{array}{c}\text { FC POLG } \\
\text { vs control }\end{array}$ & $\begin{array}{c}\text { FC tRNA-Leu } \\
\text { vs control }\end{array}$ & $\begin{array}{c}\text { FC All } \\
\text { vs control }\end{array}$ \\
\hline HSPA1A & & $1.58(1.33-1.87)$ & & $3.39(2.80-4.09)$ \\
ICAM1 & & $1.40(1.35-1.45)$ & & \\
IGFBP3 & $0.68(0.56-0.82)$ & $1.27(1.03-1.56)$ & $0.68(0.56-0.82)$ & \\
IL2RG & & $2.36(2.07-2.69)$ & & $0.58(0.38-0.89)$ \\
MT1M & & & \\
PON2 & & $2.60(1.36-4.96)$ & & \\
SIRT1 & $1.99(1.71-2.31)$ & & \\
SOD1 & $1.38(1.27-1.50)$ & & \\
SOS2 & & & & \\
TNFRSF1B & $0.67(0.59-0.76)$ & & & \\
\hline
\end{tabular}

Fold changes of stress-related genes in patients versus controls as measured by the OxyGenes ${ }^{\mathrm{TM}}$ microarrays. Fold changes were the same for glucose and galactose medium. Values are estimated fold changes for patient groups versus controls provided by the model, taking into account both the effect of the mutation and the medium. If the fold change was not significantly different for all patient groups, the fold change of the best fitting model was estimated for three groups together and provided in the 'All vs control' column. Only significant results are shown.

Total glutathione levels were measured to evaluate the oxidative status of the cell lines (figure 5.1). Univariate analysis of variance did not detect any significant difference between the groups or culture conditions. As can be appreciated from figure 5.1, there was a large variation between cell lines of patients within one group. Eight patient cell lines showed total glutathione values below the control range in the glucose situation. Additionally, the total glutathione levels in galactose were increased compared with the glucose levels in seven of these same cell lines, making them similar to control levels in galactose. On the contrary a decrease or no change at all was observed in the control cell lines or the remaining patient cell lines.

\section{Correlations between different parameters}

To search for correlations between the different parameters (ROS levels, oxidative protein damage, total GSH levels and classic antioxidant gene expression) and between these parameters and sex and age, a correlation matrix was created 
(supplementary table 5.2). Statistical significant correlations ( $p$ value $<0.05$ ) were only detected between ROS levels and CAT expression fold changes (Pearson's $r$-0.508) and protein carbonyl levels and GPX1 expression fold changes (Pearson's r 0.454).

\section{Discussion}

The goal of this study was to characterize different oxidative stress parameters in three groups of patients with a genetically characterized mitochondrial disorder caused by mutations either in the POLG1 gene or in mtDNA encoded complex I and tRNA leucine genes. Fibroblast cells predominantly use glycolysis for energy production when cultured with high glucose availability [24, 32, 33]. Hence, cell lines were forced to derive ATP from OXPHOS using glucose-free galactose medium [34]. An overview of the results is shown in table 5.6.

Table 5.5. Antioxidant gene expression for each cell line.

\begin{tabular}{|c|c|c|c|c|c|c|c|c|}
\hline \multirow{2}{*}{$\begin{array}{l}\text { Gene } \\
\text { Cell line }\end{array}$} & \multicolumn{2}{|c|}{ CAT } & \multicolumn{2}{|c|}{ SOD1 } & \multicolumn{2}{|c|}{ GSR } & \multicolumn{2}{|c|}{ GPX1 } \\
\hline & glu & gal & glu & gal & glu & gal & glu & gal \\
\hline \multicolumn{9}{|l|}{ POLG } \\
\hline $2862 S$ & & 1.37 & 2.35 & 19.05 & & 2.42 & 0.77 & 2.50 \\
\hline 06Е0703 & & 1.36 & \multicolumn{6}{|c|}{1.21} \\
\hline O0E0741 & 1.29 & & & & 1.22 & & 1.51 & 0.58 \\
\hline 3591 & & & & & & 1.46 & & \\
\hline 05E0536 & & & & & & & & \\
\hline \multicolumn{9}{|l|}{ tRNA-Leu } \\
\hline 2400 & & & 14.35 & 0.64 & 1.75 & & & 0.54 \\
\hline 1933 & & & & & & & & 0.67 \\
\hline 2830 & & & \multirow{2}{*}{\multicolumn{2}{|c|}{0.56}} & & 2.64 & & 0.42 \\
\hline 1330 & & & & & & & 0.75 & 0.51 \\
\hline
\end{tabular}

$\underline{\mathrm{Cl}}$

3765

1.40

2181

$4.11 \quad 0.54$

1.17

0.79

1682

0.61

1.29

Fold changes are given relative to the average control (glucose or galactose). Values are estimated fold changes for patients versus controls provided by the model, taking into account both the effect of the mutation and the medium. If the fold change was not significantly different for a cell line between both media, the fold change of the best fitting model was estimated for glucose and galactose together. Blanc cells indicate no ( $<10 \%$ change) or a non-significant difference compared with controls. 
Oxidative stress in patients with mutations in mtDNA encoded complex I subunits

The fibroblast cell lines with mutations in mtDNA encoded complex I subunits presented the highest ROS levels, accompanied with most oxidative protein damage (2/3 cell lines) and only few changes in antioxidant gene expression (table 5.6). Interestingly, the $\mathrm{Cl}$ cell line with the highest ROS levels was the cell line without oxidative damage, indicating adaptation to or compensation of the defect. The other two cell lines seemed less capable to adapt to or compensate for increased ROS levels, resulting in oxidative stress and damage. Possible consequences of extensive oxidative stress are cell cycle arrest, senescence and apoptosis [35]. Increased ROS levels have been detected previously in cells or tissues of patients with a genetically (nuclear [23] or mitochondrial DNA [16]) and/or functionally [4] characterized CI deficiency. The finding is not surprising as $\mathrm{Cl}$ is one of the major ROS sources in mitochondria [36]. Luo et al observed increased lipid peroxidation in fibroblasts of patients with $\mathrm{Cl}$ deficiency [37], but this was not detected in another study by Verkaart et al [15]. The only publication reporting on oxidative protein damage in $\mathrm{Cl}$ deficiency involved cybrids carrying the 3460, 11778 and 14484 Leber mutations showing increased protein carbonylation [38]. This nicely supports our results. In contrast to the lymphoblast cell lines from biochemically characterized $\mathrm{Cl}$ deficient patients with predominantly (5/6 cell lines) homoplasmic mtDNA mutations [16], we did not detect consistently increased expression of SOD, CAT, GPX and GST in our patients with a genetic defect in mtDNA Cl genes [16]. However, in their study, increased expression of the genes in the presence of elevated ROS levels was not correlated with increased expression of the corresponding proteins and they actually measured lower antioxidant activity of these enzymes, except for SOD1. In spite of the inconsistency between RNA and protein data, they concluded, mainly based on the enzyme data, that ROS levels and alteration of ROS scavenging enzymes were good parameters to assign antioxidant therapy. However, oxidative damage parameters were not examined, which in our study seemed to be an appropriate biomarker to estimate the eventual consequences of increased ROS levels in fibroblasts.

\section{Oxidative stress in patients with mutations in tRNA leucine genes}

All three cell lines with the m.3243A>G mutation (80-90\% heteroplasmy) experienced increased ROS levels, whereas the cell line with the m.3302A $>$ G mutation $(60 \%)$ did not (table 5.6). In this study with primary patient fibroblast cell lines, the lack of increased ROS levels in the m.3302A $>G$ cell line is likely due to the lower mutation percentage but a different molecular mechanism compared with the m. 3243A>G tRNALeu mutation cannot be excluded [39]. The m.3302A $>G$ and m.3243A>G tRNA-Leu mutations have been shown to increase superoxide production in homoplasmic cybrid models with deficiencies of OXPHOS complexes I, III, IV and V [40], whereas in our group complex I deficiency was most prominent, and in muscle of patients with the m.3243A $>$ G mutation [19]. Oxidative protein damage was observed in two m.3243A>G cell lines. Again, the cell line with the highest ROS levels did not show oxidative damage in galactose. For the tRNA-Leu patients, only few studies previously reported on oxidative damage. One study detected increased protein carbonylation in muscle biopsies [19] while two other studies did not observe increased oxidative damage in 
cybrids carrying the homoplasmic $3243 A>G[6,39]$ or $3302 A>G$ [39] mutations. There have been no reports on oxidative stress/damage in mutant tRNA-Leu fibroblasts so far.

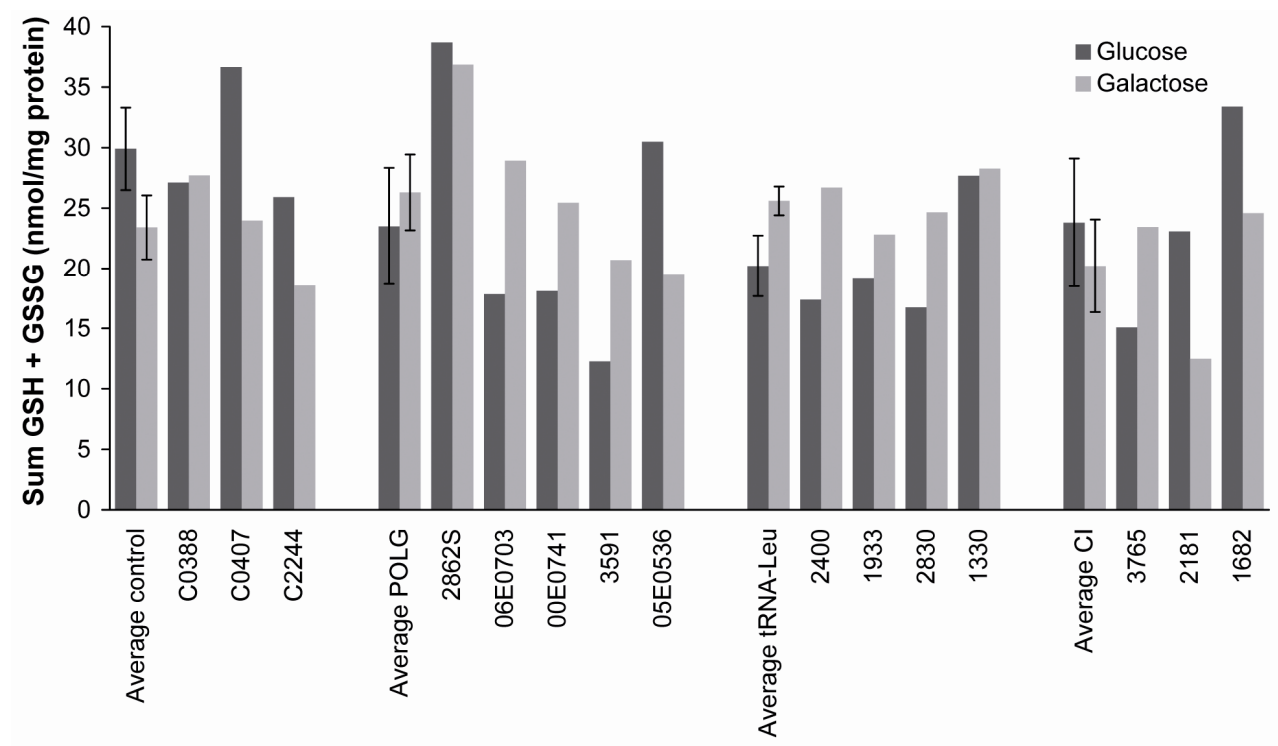

Cell line

Figure 5.1. Total glutathione (GSH+GSSG) levels.

Our results differ partly from previous studies on antioxidant enzymes in MELAS patients, reporting increased gene expression and activities of SOD, CAT and GPX, although studies are difficult to compare due to differences in cell types, mutation load and analytical approaches [5, 6, 41]. In one study, the mutation load was considerably lower ( $<45 \%$ heteroplasmy) than in our fibroblasts, and myoblast cultures were used [5]. This might indicate that antioxidant defenses are triggered at lower ROS levels in muscle-derived cells, compared with fibroblasts, to prevent accumulation of oxidative damage in post-mitotic tissues; however, ROS levels, which could support this hypothesis, were not determined in the myoblast cultures. Therefore, it is not certain that the gene expression changes resulted from increased ROS production related to the mtDNA mutations. A second study did not quantify their immunohistochemical results in muscle [41], making it difficult to judge and compare the results. Finally, a third study used homoplasmic mutation load and cybrid models [6] instead of primary patient material. Although there is a possible interference of the nuclear cancer background here, the increased expression of antioxidant genes nicely correlates with the lack of oxidative damage. Altogether, all studies point to increased ROS production, whether or not compensated by antioxidant defenses. These studies emphasize the importance of standardization and examining different aspects of oxidative stress to be 
able to compare the results of different studies, especially if heterogeneous patient populations or different model systems with different adaptive capacities are being used.

Oxidative stress in patients with mutations in the POLG1 gene

All except one POLG1 cell line showed increased ROS levels. POLG1 cell line $06 \mathrm{E} 0703$ has the same mutation as cell line 2862S, while only the latter showed increased ROS levels (table 5.6). This suggests that other factors in addition to or apart from the primary genetic defect(s) play a role in ROS levels in the POLG group (and possibly also in the other groups). This might involve intrinsic individual differences in ROS scavenging capacity due to e.g. genetic polymorphisms or environmental factors [42]. It may also be related to the random effect of pol $y$ on mtDNA stability and point mutations leading to many, random low level heteroplasmy mutations in contrast to the high heteroplasmic $\mathrm{Cl}$ and tRNA-Leu mutations. Even though the POLG and TRNA-Leu group experienced similar ROS levels, the former group seemed less vulnerable to accumulate oxidative protein damage in fibroblasts. As suggested by the multiple gene expression changes, this is possibly correlated with an adaptive activation of the antioxidant or repair systems associated with increased endogenous ROS levels in our patient lines [43]. No human cells or tissues with POLG1 mutations have been evaluated for oxidative stress parameters so far. In POLG mutator mice with proofreading deficient D268A polymerase resulting in the random accumulation of mtDNA mutations, no or only mildly increased ROS production and no oxidative damage could be detected in several tissues (liver, heart, muscle) by two groups [25, 26]. Contrastingly, a third group examining the same mice, did observe increased oxidative protein damage in the heart which could be attenuated by overexpression of mitochondrial catalase [44]. Additionally, in transgenic mice with cardiac-targeted human mutant Y955C pol $\mathrm{Y}$, affecting the polymerase domain, increased oxidative DNA damage associated with decreased mtDNA copy number was observed [27]. In concordance with the latter publications, the current study implies that ROS levels are increased in most POLG1 patients and therefore their antioxidant levels and oxidative damage markers should be followed up to supplement them with antioxidants when their endogeneous system starts failing.

\section{Increased ROS levels in the majority of primary fibroblasts of OXPHOS patients}

One of the general observations in this study is that ROS levels were increased in most patient-derived cell lines, both when cultured with glucose and galactose medium (table 5.6). The moderate increase in ROS levels in control fibroblasts due to the replacement of glucose by galactose might reflect increased basal OXPHOS-related ROS production due to the switch from glycolysis to OXPHOS for energy production. Our results indicated that ROS levels could already be increased by quite moderate OXPHOS deficiencies (e.g. Cl activity lower than $70 \%$ of the average control activity), as shown for most fibroblasts in this study, e.g. due to disturbed electron fluxes in the mutated complexes. In support of this theory, increased superoxide production was measured in fibroblasts of patients with a mutation in a nuclear DNA-encoded $\mathrm{Cl}$ gene and residual $\mathrm{Cl}$ activities up to $75 \%$ of controls [23]. A drawback is that the method 
applied cannot distinguish the exact site(s) of HEt oxidation and that the exact mechanism therefore remains speculative [23]. Other proteins that might influence the measured ROS levels are alpha-ketoglutarate dehydrogenase in the tricarboxylic acid cycle [45, 46] and NADPH oxidases (NOX) [47], all suggested to be influenced by the redox status of cells [48]. Therefore, genetically defined OXPHOS deficiencies might not only increase ROS levels by electron leakage at the OXPHOS complexes but also by altering the $\mathrm{NAD}^{+} / \mathrm{NADH}$ ratio.

\section{Adaptive processes in fibroblasts of OXPHOS patients}

Most cell lines showed increased ROS levels but no increased oxidative protein damage. Stress-induced adaptive gene expression changes, especially in the POLG1 cell lines, might account for these findings. The existence of such a delicate balance is supported by the presence of oxidative damage in one $\mathrm{Cl}$ mutant fibroblast cell line in galactose but not glucose medium, associated with higher ROS levels. The increased expression of stress-inducible HSPA1A, DNAJB1 and HSF2 in the glucose and/or galactose condition in patient cell lines pointed to a stress condition induced by the genetic defect. Heat shock proteins function as chaperones for correct folding of other proteins. The induction of these genes indicates activation of the unfolded protein response and possibly increased endoplasmic reticulum stress. Defects in protein synthesis have been associated with the $3243 A>G$ mutation previously [49]. The activation of the unfolded protein response in the patients of this study might be related to prevention or repair of oxidative protein damage. Other compensatory mechanisms such as protein regeneration and muscle regeneration have also been observed in muscle biopsies of m.3243A $>\mathrm{G}$ mutation carriers [19]. However, repair and regenerative processes are highly energy demanding. Therefore, probably also the remaining energy capacity of a defect OXPHOS system plays a role in the faith of cells. Recently, exercise training has been shown to be beneficial for improving oxidative metabolism in muscle [50] and to induce antioxidant-related adaptations, enhancing the ability to cope with oxidative stress [51]. Thus, possibly also the physical activity or training of patients plays a role in how well their antioxidant systems will be able to cope with or adapt to the increased ROS levels caused by their genetic defect.

A consistent correlation was observed between classic antioxidant genes and the other oxidative stress parameters. ROS levels were inversely correlated with CAT expression and protein carbonyl levels were correlated with GPX1 gene expression. Superoxide has been shown previously to inhibit catalase directly [52], whereas GPX1 was crucial for protection against protein oxidation in mice [53], both of which might explain the observed correlations. Therefore, the expression of these genes might serve as biological biomarkers, although this remains to be confirmed in a larger patient cohort. 


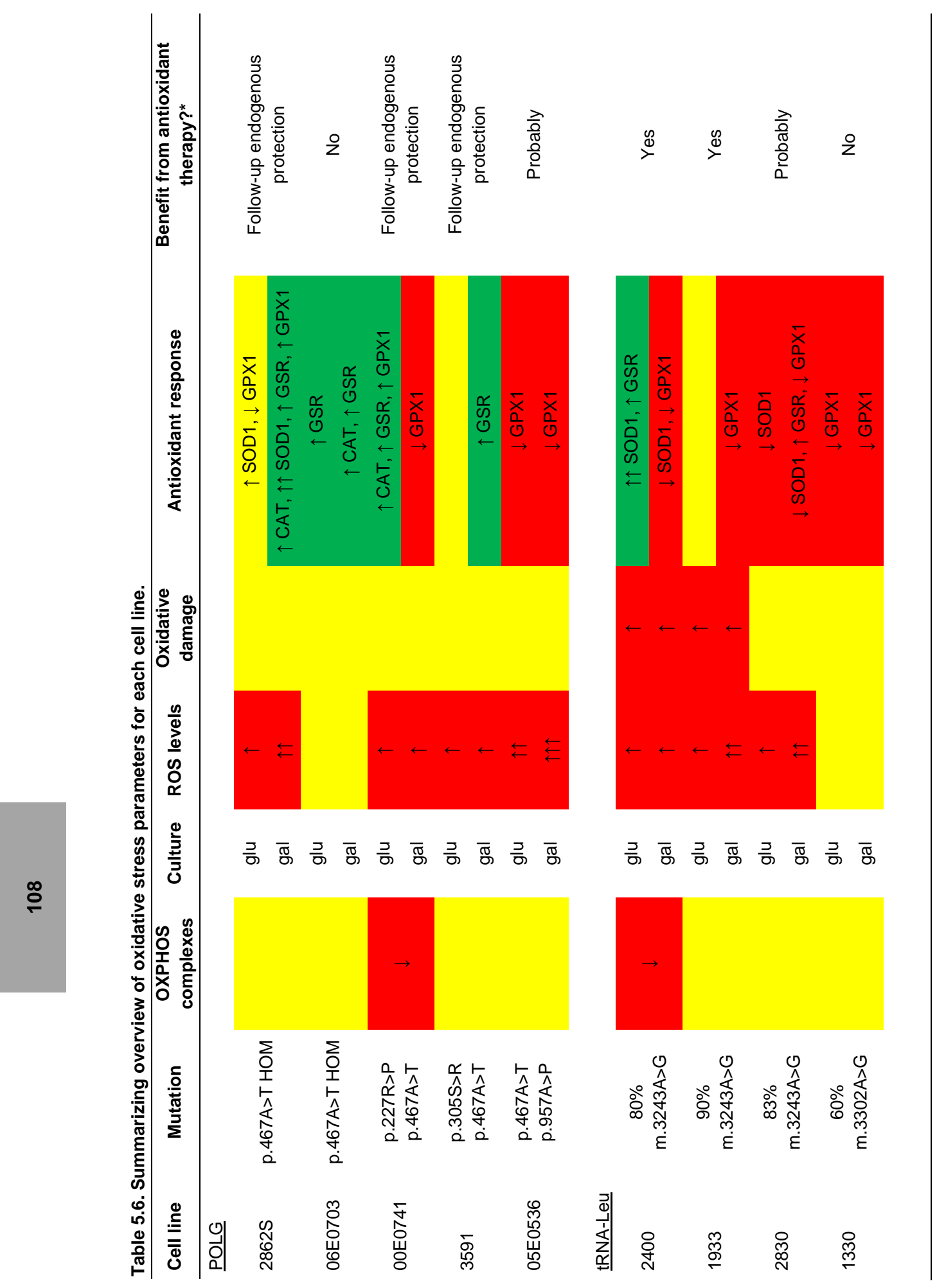




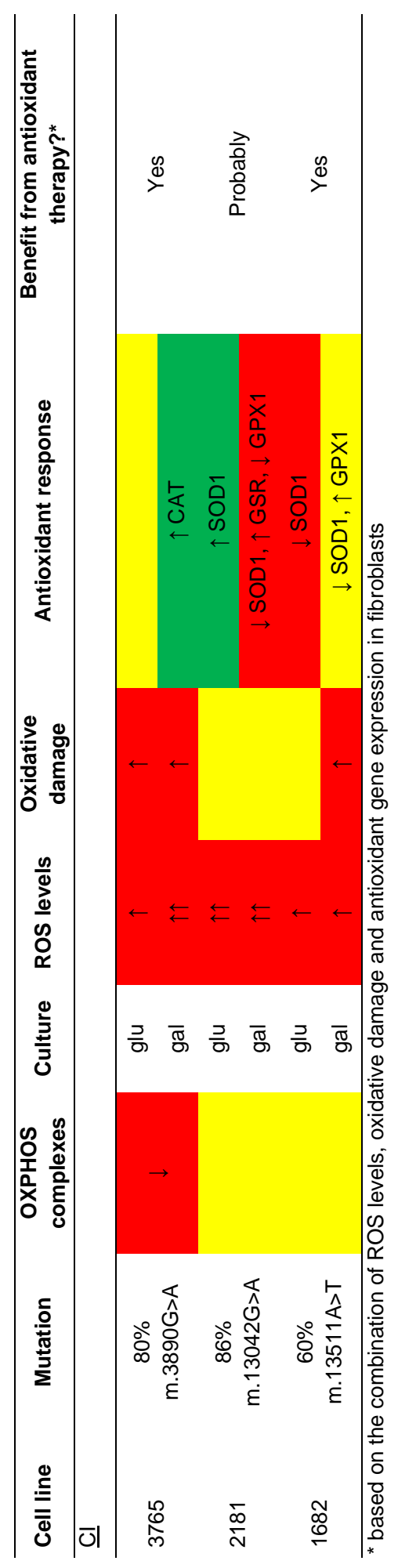




\section{Conclusions}

In this study using fibroblasts of patients with different genetically characterized OXPHOS disease, it was shown that in the majority ROS was increased and could lead to oxidative damage in different OXPHOS disorders, although not in every patientderived fibroblast. This has to be determined individually. Whether this also applies to more clinically relevant tissues, like brain and muscle of those patients, is not clear, but is not unlikely given data from literature involving other tissues [19, 54, 55] and mouse studies $[27,56]$ illustrating that fibroblasts are not the most sensitive system. Therefore, patient-derived fibroblasts can be used to identify patients with increased risk for oxidative damage, who will most likely benefit from boosting their antioxidant defense and energy capacity (training), possibly leading to patient-tailored prevention and antioxidant therapy in the future. Nevertheless, even in these patients, efficacy and toxicity of antioxidant supplementation should be evaluated carefully as side-effects can occur [57].

\section{Acknowledgements}

This work was supported by the Dutch IOP Genomics grant IGE05003 and the Interreg IV program (project EMR.INT4). We would like to thank I. Kuipers and N. Reynaert for technical expertise and help.

\section{Supplementary data}

Supplementary table 5.1. Significantly changed genes with medium effect on the OxyGenes array.

Supplementary table 5.2. Correlation matrix for ROS levels, protein carbonyl levels, total GSH levels and fold changes of classic antioxidant genes.

\section{References}

1. Floreani, M., et al., Antioxidant defences in cybrids harboring mtDNA mutations associated with Leber's hereditary optic neuropathy. FEBS J, 2005. 272(5): p. 112435.

2. Pang, C.Y., H.C. Lee, and Y.H. Wei, Enhanced oxidative damage in human cells harboring A3243G mutation of mitochondrial DNA: implication of oxidative stress in the pathogenesis of mitochondrial diabetes. Diabetes Res Clin Pract, 2001. 54 Suppl 2: p. S45-56.

3. Piccolo, G., et al., Biological markers of oxidative stress in mitochondrial myopathies with progressive external ophthalmoplegia. J Neurol Sci, 1991. 105(1): p. 57-60.

4. Pitkanen, S. and B.H. Robinson, Mitochondrial complex I deficiency leads to increased production of superoxide radicals and induction of superoxide dismutase. $\mathrm{J}$ Clin Invest, 1996. 98(2): p. 345-51. 
5. Rusanen, H., K. Majamaa, and I.E. Hassinen, Increased activities of antioxidant enzymes and decreased ATP concentration in cultured myoblasts with the 3243A-$>$ G mutation in mitochondrial DNA. Biochim Biophys Acta, 2000. 1500(1): p. 10-6.

6. Vives-Bauza, C., et al., Enhanced ROS production and antioxidant defenses in cybrids harbouring mutations in mtDNA. Neurosci Lett, 2006. 391(3): p. 136-41.

7. Wei, Y.H., et al., Oxidative stress in human aging and mitochondrial diseaseconsequences of defective mitochondrial respiration and impaired antioxidant enzyme system. Chin J Physiol, 2001. 44(1): p. 1-11.

8. Wojewoda, M., J. Duszynski, and J. Szczepanowska, Antioxidant defence systems and generation of reactive oxygen species in osteosarcoma cells with defective mitochondria: Effect of selenium. Biochim Biophys Acta, 2010. 1797(6-7): p. 890-6.

9. Balaban, R.S., S. Nemoto, and T. Finkel, Mitochondria, oxidants, and aging. Cell, 2005. 120(4): p. 483-95.

10. Powers, S.K., et al., Reactive oxygen species are signalling molecules for skeletal muscle adaptation. Exp Physiol, 2010. 95(1): p. 1-9.

11. Hawkins, C.L., P.E. Morgan, and M.J. Davies, Quantification of protein modification by oxidants. Free Radic Biol Med, 2009. 46(8): p. 965-88.

12. Sekiguchi, M. and T. Tsuzuki, Oxidative nucleotide damage: consequences and prevention. Oncogene, 2002. 21(58): p. 8895-904.

13. Bayeva, M. and H. Ardehali, Mitochondrial Dysfunction and Oxidative Damage to Sarcomeric Proteins. Curr Hypertens Rep, 2010.

14. Stark, G., Functional consequences of oxidative membrane damage. J Membr Biol, 2005. 205(1): p. 1-16.

15. Verkaart, S., et al., Mitochondrial and cytosolic thiol redox state are not detectably altered in isolated human NADH:ubiquinone oxidoreductase deficiency. Biochim Biophys Acta, 2007. 1772(9): p. 1041-51.

16. Wani, A.A., et al., Analysis of reactive oxygen species and antioxidant defenses in complex I deficient patients revealed a specific increase in superoxide dismutase activity. Free Radic Res, 2008. 42(5): p. 415-27.

17. Lu, C.Y., et al., Increased expression of manganese-superoxide dismutase in fibroblasts of patients with CPEO syndrome. Mol Genet Metab, 2003. 80(3): p. 321-9.

18. Ma, Y.S., et al., Upregulation of matrix metalloproteinase 1 and disruption of mitochondrial network in skin fibroblasts of patients with MERRF syndrome. Ann N Y Acad Sci, 2005. 1042: p. 55-63.

19. van Eijsden, R.G., et al., Termination of damaged protein repair defines the occurrence of symptoms in carriers of the m.3243A > G tRNA(Leu) mutation. J Med Genet, 2008. 45(8): p. 525-34.

20. Koopman, W.J., et al., Inhibition of complex I of the electron transport chain causes O2-. -mediated mitochondrial outgrowth. Am J Physiol Cell Physiol, 2005. 288(6): p. C1440-50.

21. Li, J., et al., Increased ROS generation and SOD activity in heteroplasmic tissues of transmitochondrial mice with A3243G mitochondrial DNA mutation. Genet Mol Res, 2008. 7(4): p. 1054-62.

22. Quinzii, C.M., et al., Respiratory chain dysfunction and oxidative stress correlate with severity of primary CoQ10 deficiency. FASEB J, 2008. 22(6): p. 1874-85.

23. Verkaart, S., et al., Superoxide production is inversely related to complex I activity in inherited complex I deficiency. Biochim Biophys Acta, 2007. 1772(3): p. 373-81.

24. Moran, M., et al., Mitochondrial bioenergetics and dynamics interplay in complex Ideficient fibroblasts. Biochim Biophys Acta, 2010. 1802(5): p. 443-53.

25. Kujoth, G.C., et al., Mitochondrial DNA mutations, oxidative stress, and apoptosis in mammalian aging. Science, 2005. 309(5733): p. 481-4.

26. Trifunovic, A., et al., Somatic mtDNA mutations cause aging phenotypes without affecting reactive oxygen species production. Proc Natl Acad Sci U S A, 2005. 102(50): p. 17993-8. 
27. Lewis, W., et al., Decreased mtDNA, oxidative stress, cardiomyopathy, and death from transgenic cardiac targeted human mutant polymerase gamma. Lab Invest, 2007. 87(4): p. 326-35.

28. van der Westhuizen, F.H., et al., Human mitochondrial complex I deficiency: investigating transcriptional responses by microarray. Neuropediatrics, 2003. 34(1): p. 14-22.

29. Rahman, I., A. Kode, and S.K. Biswas, Assay for quantitative determination of glutathione and glutathione disulfide levels using enzymatic recycling method. Nat Protoc, 2006. 1(6): p. 3159-65.

30. De Groote, D., et al., Effects of oral contraception with ethinylestradiol and drospirenone on oxidative stress in women 18-35 years old. Contraception, 2009. 80(2): p. 187-93.

31. Akaike, H. Information theory and an extension of the maximum likelihood principle. in Second International Symposium on Inference Theory. 1973. Budapest: Akadémiai Kiadó.

32. Rossignol, R., et al., Energy substrate modulates mitochondrial structure and oxidative capacity in cancer cells. Cancer Res, 2004. 64(3): p. 985-93.

33. Guillery, O., et al., Modulation of mitochondrial morphology by bioenergetics defects in primary human fibroblasts. Neuromuscul Disord, 2008. 18(4): p. 319-30.

34. Robinson, B.H., et al., Nonviability of cells with oxidative defects in galactose medium: a screening test for affected patient fibroblasts. Biochem Med Metab Biol, 1992. 48(2): p. 122-6.

35. Unterluggauer, H., et al., Senescence-associated cell death of human endothelial cells: the role of oxidative stress. Exp Gerontol, 2003. 38(10): p. 1149-60.

36. Rigoulet, M., E.D. Yoboue, and A. Devin, Mitochondrial ROS generation and its regulation: mechanisms involved in $H(2) O(2)$ signaling. Antioxid Redox Signal, 2011. 14(3): p. 459-68.

37. Luo, X., et al., Excessive formation of hydroxyl radicals and aldehydic lipid peroxidation products in cultured skin fibroblasts from patients with complex I deficiency. J Clin Invest, 1997. 99(12): p. 2877-82.

38. Beretta, S., et al., Leber hereditary optic neuropathy mtDNA mutations disrupt glutamate transport in cybrid cell lines. Brain, 2004. 127(Pt 10): p. 2183-92.

39. Maniura-Weber, K., et al., Molecular dysfunction associated with the human mitochondrial 3302A>G mutation in the MTTL1 (mt-tRNALeu(UUR)) gene. Nucleic Acids Res, 2006. 34(22): p. 6404-15.

40. von Kleist-Retzow, J.C., et al., Impaired mitochondrial Ca2+ homeostasis in respiratory chain-deficient cells but efficient compensation of energetic disadvantage by enhanced anaerobic glycolysis due to low ATP steady state levels. Exp Cell Res, 2007. 313(14): p. 3076-89.

41. Filosto, M., et al., Antioxidant agents have a different expression pattern in muscle fibers of patients with mitochondrial diseases. Acta Neuropathol, 2002. 103(3): p. 215-20.

42. Di Pietro, A., et al., Ex vivo study for the assessment of behavioral factor and gene polymorphisms in individual susceptibility to oxidative DNA damage metals-induced. Int J Hyg Environ Health, 2011. 214(3): p. 210-8.

43. Kulkarni, R., et al., Mitochondrial gene expression changes in normal and mitochondrial mutant cells after exposure to ionizing radiation. Radiat Res, 2010. 173(5): p. 635-44.

44. Dai, D.F., et al., Age-dependent cardiomyopathy in mitochondrial mutator mice is attenuated by overexpression of catalase targeted to mitochondria. Aging Cell, 2010. 9(4): p. 536-44.

45. Tretter, L. and V. Adam-Vizi, Generation of reactive oxygen species in the reaction catalyzed by alpha-ketoglutarate dehydrogenase. J Neurosci, 2004. 24(36): p. 77718. 
46. Starkov, A.A., et al., Mitochondrial alpha-ketoglutarate dehydrogenase complex generates reactive oxygen species. J Neurosci, 2004. 24(36): p. 7779-88.

47. Bedard, K. and K.H. Krause, The NOX family of ROS-generating NADPH oxidases: physiology and pathophysiology. Physiol Rev, 2007. 87(1): p. 245-313.

48. Pendyala, S. and V. Natarajan, Redox regulation of Nox proteins. Respir Physiol Neurobiol, 2010. 174(3): p. 265-71.

49. Chomyn, A., et al., MELAS mutation in mtDNA binding site for transcription termination factor causes defects in protein synthesis and in respiration but no change in levels of upstream and downstream mature transcripts. Proc Natl Acad Sci U S A, 1992. 89(10): p. 4221-5.

50. Hassani, A., R. Horvath, and P.F. Chinnery, Mitochondrial myopathies: developments in treatment. Curr Opin Neurol, 2010. 23(5): p. 459-65.

51. De Lisio, M., et al., Exercise training enhances the skeletal muscle response to radiation-induced oxidative stress. Muscle Nerve, 2011. 43(1): p. 58-64.

52. Kono, Y. and I. Fridovich, Superoxide radical inhibits catalase. J Biol Chem, 1982. 257(10): p. 5751-4.

53. Cheng, W., et al., Selenium-dependent cellular glutathione peroxidase protects mice against a pro-oxidant-induced oxidation of $N A D P H, N A D H$, lipids, and protein. FASEB J, 1999. 13(11): p. 1467-75.

54. Umaki, Y., et al., Apoptosis-related changes in skeletal muscles of patients with mitochondrial diseases. Acta Neuropathol, 2002. 103(2): p. 163-70.

55. Katayama, Y., et al., Accumulation of oxidative stress around the stroke-like lesions of MELAS patients. Mitochondrion, 2009. 9(5): p. 306-13.

56. Quintana, A., et al., Complex I deficiency due to loss of Ndufs4 in the brain results in progressive encephalopathy resembling Leigh syndrome. Proc Natl Acad Sci U S A, 2010. 107(24): p. 10996-1001.

57. Bjelakovic, G., et al., Mortality in randomized trials of antioxidant supplements for primary and secondary prevention: systematic review and meta-analysis. JAMA, 2007. 297(8): p. 842-57. 


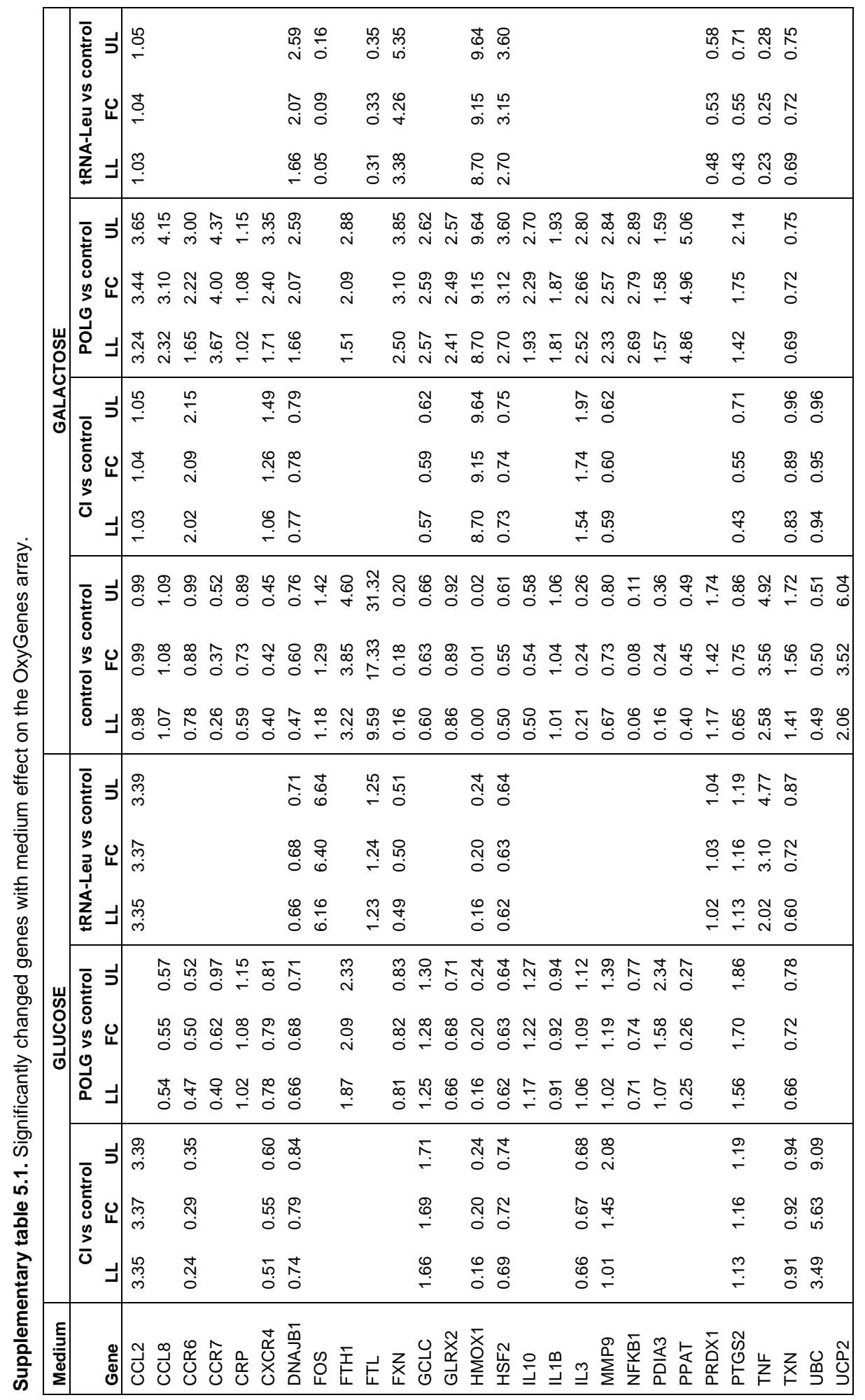


Supplementary table 5.2. Correlation matrix

\begin{tabular}{|c|c|c|c|c|c|c|c|}
\hline & $\begin{array}{l}\frac{\omega}{\Phi} \\
\frac{\partial}{\infty} \\
\stackrel{\infty}{\simeq} \\
\simeq\end{array}$ & 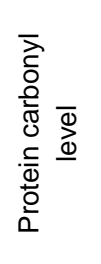 & 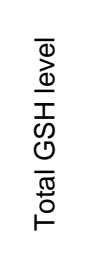 & 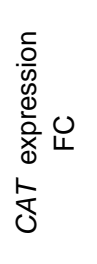 & 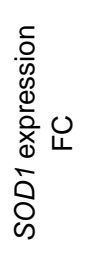 & 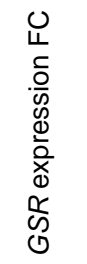 & $\begin{array}{l}\frac{5}{0} \\
\text { कू } \\
\varrho \\
\frac{0}{0} \\
\frac{1}{x} \\
0 \\
\frac{1}{x} \\
\frac{1}{0}\end{array}$ \\
\hline \multicolumn{8}{|l|}{ ROS levels } \\
\hline Protein carbonyl level & -0.158 & & & & & & \\
\hline Total GSH level & -0.093 & -0.032 & & & & & \\
\hline CAT expression FC & -0.508 & -0.197 & -0.005 & & & & \\
\hline SOD1 expression FC & -0.001 & -0.050 & 0.246 & 0.227 & & & \\
\hline GSR expression FC & 0.258 & -0.170 & -0.120 & -0.160 & 0.400 & & \\
\hline GPX1 expression FC & 0.225 & 0.454 & 0.046 & -0.177 & 0.327 & 0.234 & \\
\hline
\end{tabular}

Correlation matrix for ROS levels, protein carbonyl levels, total GSH levels and fold changes of classic antioxidant genes. The Pearson correlation values produced by SPSS are shown; significant correlations are depicted in bold. 



\section{Chapter 6}

\section{General Discussion}


Mitochondrial enchephalomyopathies are a clinically and genetically highly heterogeneous group of diseases. They are also referred to as oxidative phosphorylation (OXPHOS) disorders, because of the causative role of deficiencies of the OXPHOS system in the pathology of most cases. The pathophysiology of these disorders is only partly understood and efficient therapies are not available for the vast majority of patients. The aims of this thesis were:

1. to facilitate diagnostics of mtDNA-based OXPHOS disorders by determining the nonpathogenic variation of mtDNA in the general population;

2. to gain knowledge on primary and secondary biological processes involved in OXPHOS disorders which have an impact on prognosis and therapy;

3. to explore the extent to which different pathogenic mtDNA and nuclear DNA mutations induce oxidative stress and the consequences of this in a cell line model.

The general discussion elaborates on the most significant findings and models and puts them in a broader perspective of strategies to unravel pathophysiology and evaluate therapy.

\section{Mitochondrial DNA: evolution and disease}

The mitochondrial DNA (mtDNA) codes for 13 subunits of complex I, III, IV and V of the OXPHOS chain and 22 tRNAs and 2 rRNAs necessary for mRNA translation. Therefore, it is not surprising that mtDNA mutations or variants can affect OXPHOS function. Some variants cause disease themselves but others only influence the risk of developing disease or affect the course of the disease (so-called risk factors and modifiers). There are also neutral variants that do not have disease-related consequences. An additional factor in determining the effect of variants is the multicopy nature of the mtDNA with a threshold of expression for heteroplasmic pathogenic mtDNA mutations. In order to understand the presence and the consequences of mtDNA variants, it is necessary to understand how mtDNA variants arise, how they are maintained and why some variants are tolerated whereas others are not.

According to the endosymbiotic hypothesis, eukaryotic mitochondria evolved from endocytosed aerobic bacteria more than 1 billion years ago, when oxygen entered the atmosphere. During eukaryotic evolution, most (but not all) of the bacterial genome encoded genes have been transferred to the nuclear genome, leading to the present dual genetic control of the OXPHOS system [1]. Interestingly, the number of transferred genes differs between eukaryotic species as exemplified in table 6.1. Although in vertebrates there is an almost invariant gene content, other species show considerable mtDNA plasticity, mostly attributable to differences in the number of tRNA genes [2]. This suggests some form of stabilization in more evolved species and a flexibility of eukaryotic mitochondria regarding their ability to import tRNAs from the cytoplasm. Even though the number of protein coding genes is rather stable, loss of ATP8 was shown in five evolutionary-distant fast-evolving taxa (Nematoda, Rotifera, Chaetognatha, Platyhelminthes and Bivalvia). This might indicate that ATP8 is 
'dispensable' in the mtDNA [2] and might also have consequences for the tolerance of variation within this gene.

Table 6.1. Genes encoded by the mtDNA in eukaryotic species.

\begin{tabular}{lcc}
\hline Species & Number of mtDNA genes & Number of protein coding genes \\
\hline H. sapiens (human) & 37 & 13 \\
M. musculus (mouse) & 37 & 13 \\
G. gallus (chicken) & 37 & 13 \\
D. rerio (zebra fish) & 37 & 13 \\
D. melanogaster (fruit fly) & 37 & 13 \\
P. megacephalus (frog) & 35 & 11 \\
C. elegans (worm) & 36 & 12 \\
S. cephaloptera (worm) & 13 & 11 \\
A. aurita (jelly fish) & 19 & 15 \\
M. occidentalis (mite) & 53 & 21 \\
S. cerevisiae (yeast) & 46 & 19 \\
A. thaliana (plant) & 131 & 117 \\
\hline According to NCBI Genome. & &
\end{tabular}

The mtDNA has a higher mutation rate compared to the nuclear DNA. Presumably due to the proximity of the mtDNA to the electron transport chain, a major ROS production site, and the lack of protective histones, the mtDNA is more prone to oxidative damage $[3,4]$. Although originally it was suggested that mitochondria have no or only a limited DNA repair capacity [5], efficient base excision repair in mitochondria was detected afterwards [6,7]. Furthermore, mitochondrial dynamics, characterized by mitochondrial fusion and fission, has been shown to be important for mitochondrial genomic stability through intramitochondrial exchange of mutant mtDNA and stimulation of autophagy of damaged mitochondria [8]. However, the uniparental (maternal) inheritance, the mitochondrial bottleneck (random unbalanced partitioning of the cytoplasm during gamete formation) and possibly low recombination, lead to accumulation of mutations that escape the repair machinery [9] even though their original goal might be the maintenance of mitochondrial genomic integrity [10]. This is not necessarily negative, as the genetic variation is the driving force of evolution, providing a species the possibility to adapt to environmental changes. For example, there is evidence of natural selection on mtDNA variants by temperature $[11,12]$. When the human population started expanding and migrated from tropical Africa to more northern and colder climates, variants that decrease coupling efficiency and ATP production but increase heat production would be advantageous for survival [11,12]. A correlation between two non-synonymous mtDNA variants in ND3 and ATP6 and temperature has been described recently [11]. Additional evidence for an adaptive role of ancient mtDNA polymorphisms comes from the presence of the same mtDNA variant on different mtDNA haplogroup backgrounds [13]. Contrastingly, the same beneficial ancient 
mtDNA adaptations might be correlated with complex bioenergetic disorders related to the sedentary life-style and increasing age of the human population today (e.g. obesity, diabetes, cardiovascular disease) [12,14]. Obviously, pathogenic mutations are under strong negative selection to prevent fixation of the mutation in the population. No advantage for disease-causing mtDNA mutations has been observed at the population level [14]. Altogether, after ages of evolution, the human mtDNA sequence will consist of conserved positions, which are identical among most species, and variable positions. Mutation of conserved positions will probably be associated with (in utero) mortality and disease (pathogenic mutations) while mutation of variable positions will have minor consequences and will be detected more frequently in the human population.

To study the strength and consequences of natural/purifying selection on mtDNA variation, offspring of the so-called mtDNA mutator mouse was studied [15]. The mutator mice accumulate high levels of mtDNA mutations randomly across their mtDNA due to their proofreading deficient polymerase gamma $[15,16]$. However, already after a few generations, a strong selection against non-synonymous changes in protein coding was reported. The strongest selection was observed in genes with high sequence conservation (COX1, COX2). Similar results were obtained for selective forces on the human mtDNA when resequencing the mitochondrial genome of 730 subjects (chapter 2). In agreement with the presumed evolutionary dispensability, also in this study ATP8 was among the genes with most tolerated variation. Until recently, the study of mtDNA and nuclear DNA variants on a large scale by PCR, DHPLC analysis and/or Sanger sequencing was laborious and time-consuming. Now, the use of next generation whole genome sequencing leads to high throughput analysis of whole genomes and will lead to a detailed map of both genomes. This map will consist of positions that are targeted by tolerated polymorphisms (occurring in healthy individuals) or pathogenic mutations (in diseased but not healthy individuals) or positions that never show variation. The latter situation probably points to mutations that are not compatible with life and will never be detected. Detailed and complete knowledge on tolerance and natural selection in large numbers of individuals will provide valuable information for evaluating the pathogenicity of DNA variants in patients and their families. Furthermore, as mitochondria have been implicated to play a role in a plethora of diseases (e.g. diabetes, Alzheimer's disease and cancer), the significance of mtDNA variation in these pathologies can also be judged more adequately. In addition to in silico predictions, functional assays can be applied for those pathogenic variants leading to enzyme deficiencies. Cytosolic hybrids (cybrids) carrying the mutant mtDNA in a cancer nuclear background (e.g. osteosarcoma) can be used for this purpose [17-19]. However, this approach does not apply to variants with subtle defects with no measurable effect on enzyme activities. 


\section{Models to study pathophysiology and/or test compounds: suitability/applicability}

When disease-causing mutations are identified, the pathogenic mechanism has to be unraveled in order to investigate potential therapeutic strategies. There will be no single model able to deal with all follow-up investigations and high-throughput analyses in cell lines and simple model organisms will be complemented with studies in more laborious mammalian models. The same applies for intervention studies. Several potential models exist, including human (post-mitotic tissue), human primary cell culture, rodents and small animals (e.g. Caenorhabditis elegans [worm], Drosophila melanogaster [fruit fly], Danio rerio [zebrafish]). Each of the models has its own advantages, disadvantages and applications (figure 6.1).

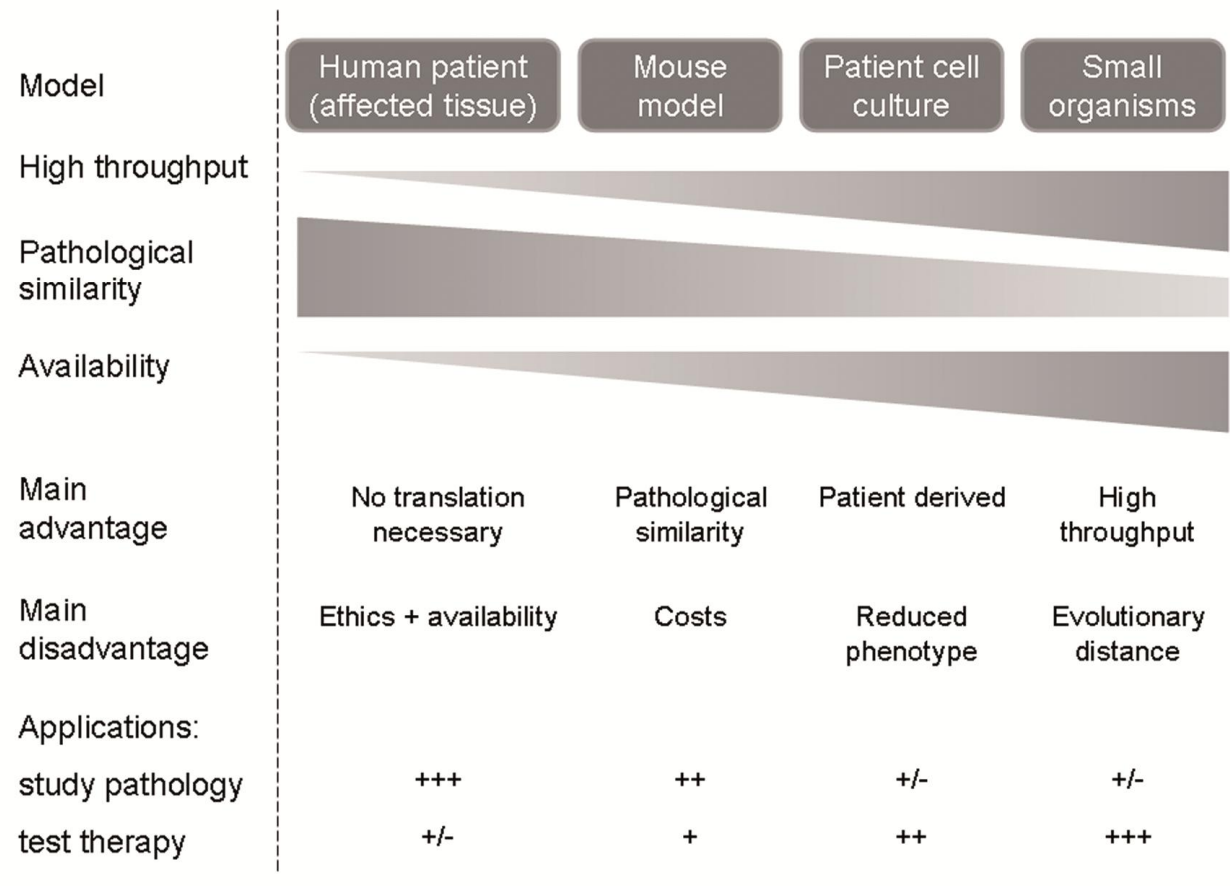

Figure 6.1. Different models to study inherited disorders with characteristics and applications.

For pathophysiological studies, affected tissues from patients would allow the most relevant and specific disease processes to be identified. However, for diseases affecting non-dividing tissues (e.g. brain), the availability and amount of material is limited. In chapter 3, we circumvented this by studying morphological, genetic (mtDNA) and gene expression changes in muscle of POLG1 patients to unravel biological processes involved in POLG1 pathogenesis. Gene expression analysis in muscle hinted towards a possible association of ROS production and apoptosis with patient phenotypes, which was subsequently confirmed in liver and brain of part of the patients. 
This study demonstrates how only mildly sub-pathologically affected but better available tissues (such as muscle) can be the surrogate tissue for the identification of pathological processes in more severely affected tissues (such as brain and liver).

Due to the limited availability of post-mitotic patient material, primary cell cultures of dividing patient tissues (e.g. fibroblasts) are often used to study pathophysiology and to test therapies in vitro [20, 21]. Although this model type allows for high-throughput in vitro screening assays, the complex in vivo interactions and physiological conditions are not comparable with in vitro conditions and often the consequences of the primary defect are less outspoken, especially in the case of OXPHOS disorders [22], where e.g. fibroblast can switch from oxidative to glycolytic energy production. Furthermore, it is important to check the genetic and biochemical stability of the cell lines over time in case they are used as tester panels for compound screening. A panel of fibroblasts cell lines of complex I deficient patients was characterized for this purpose (chapter 4). The primary goal was to characterize a complex I deficient cell culture system that could be used for high-throughput screening of small compounds for beneficial effects on complex I activity and energy production. The use of fibroblasts was complicated by the mainly $(\sim 70 \%)$ glycolytic energy production in fibroblasts under standard (glucose) culture conditions [23]. To induce OXPHOS-derived (oxidative) energy production, and thus stress the primary OXPHOS defect, fibroblast cell lines were cultured in glucoselacking galactose-containing medium in the presence of glutamine (an essential amino acid for cell growth [24]). Although in HeLa cells, it has been shown that ATP production in galactose medium occurs mainly (98\%) through glutaminolysis [25], others did not detect a glutamine dependency of fibroblasts in glucose and galactose medium [26]. Nevertheless, possible energy production through other non-glytolytic pathways has to be kept in mind. Only minor gene expression changes and no signs of mtDNA instability (large scale rearrangements and copy number) were detected, suggesting that cultured complex I deficient fibroblasts have an inherent genetic stability. This makes these parameters less suitable for testing interventions, but they can be used to check stability of the model in time. Other parameters such as biochemical indicators (e.g. posttranslational modification of pyruvate dehydrogenase), mitochondrial morphology, mitochondrial membrane potential and calcium fluxes [2729] have been tested as well and are better indicators to test the effect of adding compounds to the cell culture medium (B. Wieringa and $P$. Willems, personal communication). The primary patient cell cultures are not only suitable for manipulating the primary defect of complex I deficiency, they can also be used to explore secondary manifestations of OXPHOS deficiency, like for example oxidative stress, which is considered a major contributing factor to OXPHOS pathology. By an in-depth analysis of the level of oxidative stress in different genetically characterized OXPHOS patients, we were able to identify patients with increased ROS levels and evaluate their risk for acquiring oxidative damage in relevant tissues (chapter 5). This information gives an indication which patients will be more likely to benefit from antioxidant therapy.

However, biological processes or responses identified by in vitro assays should be carefully translated to the patients. Especially when screening compounds or drugs, one must keep in mind that the in vitro results do not always correlate with in vivo 
effects. Validation of candidate compounds in animal models is necessary to examine their pharmacokinetic and toxic properties before going into clinical trials. For some compounds already on the market, like ROS-scavenger or stimulators of mitochondrial biogenesis, the toxic properties have been established, but the positive effect should be carefully judged. This may be difficult as the patients are often unique and grouping is not possible, implying that drug administration is based on individual evaluation and not on a group effect. For better insight in this, mice models are often used because of the high homology between the human and murine genes but also due to the similar phenotypes they develop. For example, mice expressing mutant proofreading deficient D257A polymerase gamma [16, 30] and mice with complex I deficiency by knocking out (KO) or mutating complex I subunit NDUFS4 [31, 32] have been created. The POLG mutator mice presented with increased accumulation of mtDNA mutations and deletions and premature ageing-related phenotypes (e.g. reduced fertility, heart enlargement, sarcopenia, hearing loss) [16, 30]. Some of these features also occur as a phenotype in POLG1 patients [33]. NDUFS4-KO mice manifested a lethal phenotype, similar to the Leigh-like symptoms in human patients carrying NDUFS4 mutations [31]. Heterozygous dominant-negative mutant NDUFS4 mice were viable and also presented with biochemical changes similar to human Leigh patients [32]. Nevertheless, it is obvious that strain specific differences exist and that it is not possible to make a mouse for each complex human phenotype. The main disadvantages of mouse or other higher organism models are the high costs and the laborious and timeconsuming nature of the experiments. In finding a balance between a high-throughput analysis and matching phenotypes, small animal models come into sight. These vertebrate $(D$. rerio) or invertebrate animals $(D$. melanogaster, $C$. elegans) usually have a rapid development, are easy to manipulate genetically and can be phenotyped in a high-throughput fashion. Mutants are often available and compounds can be administered by adding directly to the food, medium or water, making these models suitable for the study of pathophysiology as well as compound screening. Their main disadvantage is their evolutionary distance to humans and the fact that not all of these small organisms contain the organs that are affected in human disease. In the end, there is not a single model optimal for every application. Therefore, the most appropriate mode of action would be to use the models interdependently in a continuum, exploiting all of their individual advantages (figure 6.1).

\section{Pathological processes in genetically characterized OXPHOS disorders}

The clinical symptoms/syndromes associated with OXPHOS dysfunction and their ages of onset are extremely variable. There is often no clear genotype:phenotype correlation [34]. One may say that the step from gene to phenotype is too big and 'genomes speak biochemistry, not phenotypes' [35] but even that is not always straightforward in case of OXPHOS disorders. Patients with exactly the same mutation(s) can have different biochemistry results in muscle [36], which challenges the concept of monogenic disorders and suggests that in mitochondrial disorders genetic complexity is the rule. During the last decades, the molecular basis of OXPHOS pathology has been 
intensively studied. This research has been primarily focused on genetically characterized OXPHOS patients. However, it should be emphasized that half of the patients with a diagnostic OXPHOS deficiency do not have a genetic diagnosis yet [37]. Therefore, family studies using linkage analysis and/or next generation or exome sequencing are applied to identify new genes associated with mitochondrial function or reveal new OXPHOS-related functions for a previously known gene. In rare cases, this approach can lead from a genetic mutation directly to therapy (e.g. CoQ10 supplementation for patients with CABC1 [38] or ETFDH [39] mutation). However, most often, the molecular consequences of a novel mutation are not so straightforward and primary and secondary consequences have to be unraveled to identify targets for therapeutic interventions.

The primary consequence of an OXPHOS disorder is generally considered to be the inability of mitochondria to supply sufficient ATP to meet cellular needs, which often becomes manifest when large amounts of energy are required, for example in case of an infection [40]. However, different gene defects lead to OXPHOS deficiency and disease in different ways. There are mutations in the protein-coding genes of the OXPHOS system itself, in genes coding for OXPHOS system assembly factors, in genes responsible for the replication and maintenance of the mtDNA and in genes more indirectly related to OXPHOS function. Furthermore, the negative effect on tissues or organs relates also to their dependence on OXPHOS instead of glycolysis for energy supply and the residual capacity they have. A summary of common processes in OXPHOS patients, partly identified and described in this thesis, is presented in figure 6.2. Interventions directed at these processes should be beneficial for the larger group of patients.

\section{Bioenergetics}

Increased lactate levels in POLG1 patients (chapter 3) and in OXPHOS patients in general [41], indicate a metabolic switch from OXPHOS to glycolysis derived ATP production and pyruvate to lactate oxidation for $\mathrm{NAD}^{+}$regeneration. Increased concentrations of lactate and tricarboxylic acid cycle intermediates are frequently encountered in patients with OXPHOS disease $[42,43]$ as a measure of disturbed bioenergetics. Reinecke et al recently reviewed the limited literature on RNA expression changes of metabolic genes in a variety of disease phenotypes and models for OXPHOS deficiency [44]. Even though a disturbance of energy metabolism genes is most often apparent in OXPHOS patients [40], expression changes of the genes involved in the different studies are highly diverse and inconsistent [44]. This is a further reflection of the extreme heterogeneic pathogenicity of the disorders, illustrating that multiple pathways and processes are playing a role. Characterization of gene expression changes, contributing to disease manifestation and progression, at the individual level is important to obtain more insight in personal consequences of and adaptations to OXPHOS deficiency. 


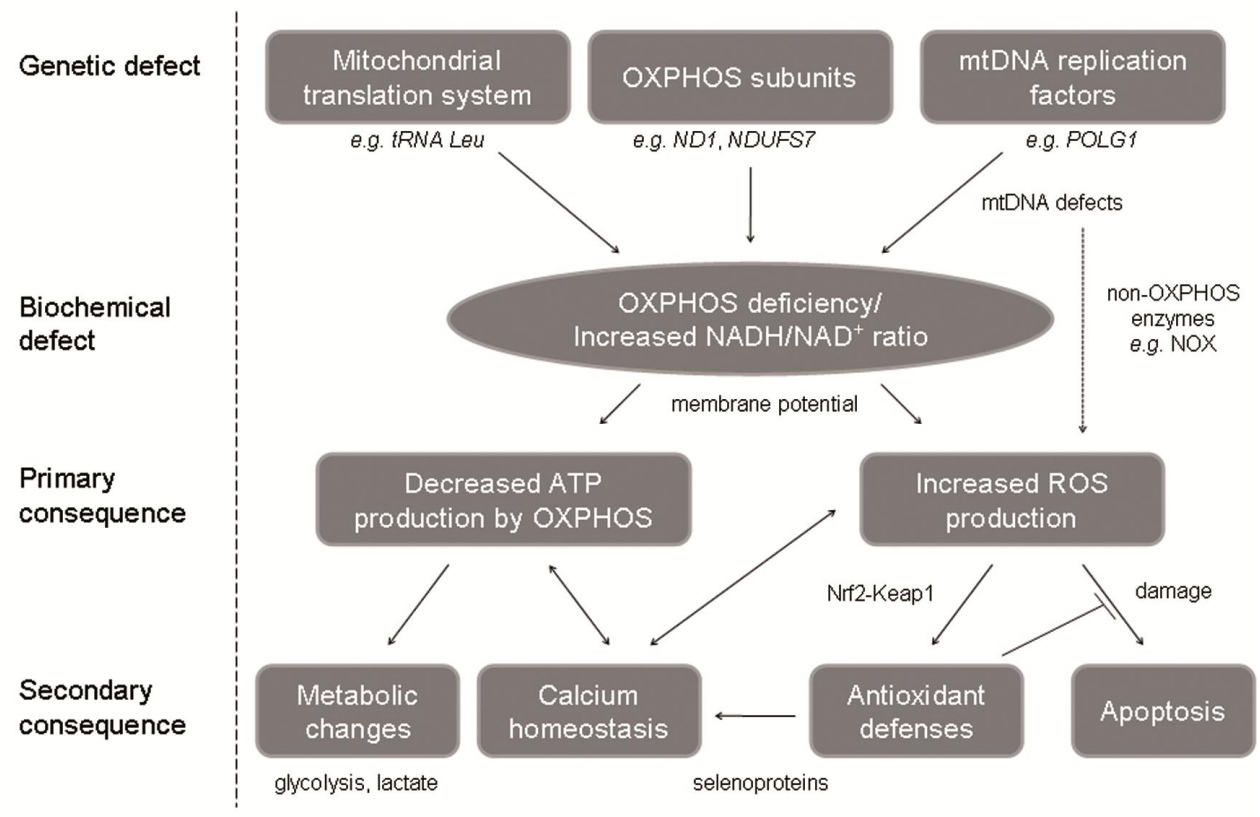

Figure 6.2. Pathophysiological processes involved in OXPHOS disease.

Mitochondrial ATP production is essential for transport of key metabolites across membranes. Cellular ionic calcium is an important second messenger, regulating many cellular processes with variable spatial and temporal dynamics [45]. Calcium homeostasis, which is normally maintained within a very narrow range around $\sim 100 \mathrm{nM}$ [45], has often been shown to be aberrant in mitochondrial diseases [46-48]. Impaired sequestration of calcium in intracellular calcium stores (mitochondria, sarcoplasmic/endoplasmic reticulum) can be secondary to ATP depletion [28] and ROS [49] due to their effect on the ATP-dependent $\mathrm{Ca}^{2+}$ pumps. Calcium has been shown to be a physiological stimulator of mitochondrial OXPHOS function, although at high concentration it changes to a pathological enhancer of ROS production [50]. Other potential pathological effects following deregulated calcium homeostasis are the opening of mitochondrial permeability transition pore, resulting in cytochrome $c$ release and apoptosis [50]. Recently, the protein responsible for mitochondrial calcium pumping, the mitochondrial calcium uniporter (MCU), was identified and characterized $[51,52]$. Future studies are necessary to establish the exact mechanism and the regulation of mitochondrial calcium transport by MCU.

OXPHOS defects are also associated with changes in the mitochondrial membrane potential $[53,54]$. Altered mitochondrial membrane potential influences mitochondrial dynamics (fission and fusion) [55]. Depolarized mitochondria are not able to fuse with the network after fission and are targeted for autophagy/mitophagy [56]. This bioenergetic quality control mechanism has to monitor that dysfunctional mitochondria are selectively removed from the mitochondrial network. Increased stimulation or 
activation of autophagy has been described for mtDNA point mutations [57] and deletions [58] before and are expected to apply also for other OXPHOS disorders with altered mitochondrial membrane potential. The processes mentioned above are generally reported in OXPHOS disorders. Nevertheless, specific gene mutations can lead to more specific metabolic consequences such as reduced flavine adenine dinucleotide (FAD) binding due to ACAD9 mutation [59], (secondary) CoQ10 deficiency due to ETFDH deficiency associated with ETFDH mutation [39] or mutation of $C A B C 1$, involved in CoQ10 synthesis [38].

\section{Oxidative stress and apoptosis}

\section{ROS production}

ROS production has predominantly been considered a secondary factor following dysfunction of one of the OXPHOS complexes (especially complex I or III [60,61]) or changes in the mitochondrial membrane potential $[62,63]$. Fibroblasts of patients with a POLG1, mitochondrial tRNA Leu or mtDNA encoded complex I subunit mutation showed increased ROS production with a moderate OXPHOS deficiency (chapter 5). This could indicate that moderately decreased (lower than 65\% activity of average control) OXPHOS function already increases ROS levels. In support of this theory, at least for complex I deficiency, increased superoxide production was measured in fibroblasts of patients with a mutation in a nuclear DNA-encoded $\mathrm{Cl}$ gene and residual $\mathrm{Cl}$ activities up to $75 \%$ of control [61]. However, the methods applied (HEt oxidation) do not distinguish the exact site(s) of ROS production [61] and therefore, other proteins might be responsible for the increased ROS levels measured. Genetic OXPHOS defects have been associated with a shift in the intramitochondrial NAD $/ \mathrm{NADH}$ ratio towards NADH $[44,64]$. This can stimulate ROS production (superoxide and $\mathrm{H}_{2} \mathrm{O}_{2}$ ) by alpha-ketoglutarate dehydrogenase in the tricarboxylic acid cycle $[65,66]$. The NADPH oxidases (NOX) are another class of proteins that mainly function in ROS production [67] and have also been suggested to be regulated by the redox status of cells [68]. Therefore, ROS should also be considered as a pathological process in genetically characterized OXPHOS patients without diagnostic OXPHOS deficiency in fibroblasts.

One therapeutic strategy that is often applied is antioxidant supplementation to scavenge increased ROS. However, up to now, the efficacy of such therapy has been highly anecdotic as patients have not been carefully evaluated for amount and origin of ROS production. The most recent evidence of a positive therapeutic effect of antioxidants for mitochondrial disease comes from a small double-blind cross-over study involving 27 mitochondrial patients and 42 controls showing that a 30-day supplementation with a cysteine donor reduced exercise-induced oxidative damage levels in blood [69]. The cell culture models established in this thesis (chapter 4 and 5) enable detailed analysis of the ROS factor in the pathology observed and could be used to measure the (beneficial) effects of soluble cell permeable antioxidant compounds by simply adding them to the cell culture and monitoring the characterized parameters. Future addition of measurements related to cellular repair capacity to this pipeline would be relevant since repair is highly energy dependent. These studies might reveal differences in oxidative damage in cell lines with similar ROS levels and antioxidant defenses and ways to prevent and treat them. Nevertheless, although 
difficult in a small and diverse patient group as this, the effect of antioxidant therapy should be evaluated critically as existing evidence also suggests a harmful effect, i.e. increased mortality, of particular antioxidant supplementation in the general population [70]. Our cell culture model can actually help in selecting patients to enroll in such an evaluation study.

\section{Antioxidant defense}

ROS have also a central physiological signaling character, inducing antioxidant pathways. This was observed by the induction of the Nrf2-Keap1 pathway (chapter 4) and the changed gene expression of classic antioxidant genes (SOD1, GPX1, GSR; chapter 5) in fibroblasts with increased ROS levels. The Nrf2-Keap1 pathway is one of the major redox-sensitive signaling systems in the cell and acts as a molecular sensor of disturbances in cellular homeostasis [71]. Activation of Nrf2 signaling was described previously in fibroblasts of patients with a mutation in the mitochondrial ATP6 gene [72], indicating a potential general therapeutic relevance for OXPHOS diseases. The Nrf2Keap1 pathway has been mentioned before as a therapeutic target for neurovascular protection in stroke [73] and has been shown to ameliorate neurodegenerative phenotypes in a Drosophila model of Parkinson's disease [74]. However, care should be taken when stimulating this pathway since deregulation of the Nrf2-Keap1 system has been implicated in carcinogenesis [75]. The consequences of modulating Nrf2, but preferentially also of more specific downstream targets can be evaluated using the cell culture models described in this thesis before translating it to animal models and eventually human patients.

Whereas the effectiveness of activation of the antioxidant system against the accumulation of oxidative damage has been shown in chapter $\mathbf{5}$, increasing antioxidant defenses can be detrimental for other cellular functions. This was shown by the preferential expression of selenoproteins involved in (oxidative) stress reduction (thioredoxins, SelK) which probably led to decreased expression of multiple other selenoproteins (chapter 4). Some of these proteins have been associated with the control of calcium fluxes (e.g. SelN, SelK, SelM) [76-78]. The decreased gene expression of these proteins in our analysis provides a new hypothesis for the decreased calcium fluxes measured previously in the studied patient cell lines [28, 29]. In these studies, decreased endoplasmic reticulum (ER) calcium content and slower calcium removal from the cytosol were observed [28]. Apart from the hypothesized role of decreased ATP delivery [28], the associations of SeIN [77] and SelK [78] with the ER membrane and its receptors point to a contribution of selenoproteins to the disturbed calcium homeostasis. Furthermore, the expression of selenoproteins is dependent on the cellular selenium status [79] and therefore, monitoring and adjusting selenium levels might eventually prove to be a new therapeutic lead for $\mathrm{Cl}$ deficient patients. The beneficial effect of selenium has also been reported for mtDNA defects [48] and therefore, this pathway is probably implicated in OXPHOS defects in more general. 


\section{Apoptosis}

The process of programmed cell death, or apoptosis, plays an important role in the regulation of normal cell populations and focal apoptosis is involved in many normal embryonic processes such as the development of organs and digits [80]. However, when apoptosis is triggered abnormally in post-mitotic tissues, the functionality of those tissues might become compromised. When antioxidant systems fail and cellular (oxidative) damage is beyond repair, apoptosis is often triggered. This was observed in liver and brain, the most affected tissues, of patients with POLG1 mutations (chapter 3). Therefore, loss of non-replaceable cells by apoptosis can be regarded as a marker of disease severity and/or progression in POLG1 pathogenesis and possibly in other OXPHOS diseases [81].

\section{Therapeutic considerations}

As a consequence of the extreme clinical, biochemical and genetic diversity in patients, it is unlikely that one single therapy could treat all OXPHOS diseases [82]. The current rationale is that slightly increasing compromised OXPHOS activity above a certain threshold may already be beneficial, even if you stimulate a dysfunctional system. Stimulation of mitochondrial biogenesis by for example controlled exercise training is currently suggested as a general approach in patients with OXPHOS disease [83]. Although the initial feeling might be that rest is better for an already compromised muscle, controlled training has been shown to induce muscle regeneration in patients with mtDNA disease from satellite cells with generally lower mutation levels, to maximize muscle function and to prevent disuse atrophy [83, 84]. Nevertheless, additional, more specific treatments will be required for patients in a form of 'personalized medicine'. Pathological processes should be characterized in each OXPHOS patient, leading to a patient profile that indicates which treatment(s) best suit(s) that patient. For example, antioxidants should only be administered to patients that really suffer from oxidative damage and would therefore benefit from this kind of therapy. Additionally, when drugs are administered to patients, it should be proven that the drug reaches the affected tissue(s). For most organs (liver, muscle), this usually does not seem to be a problem, but when the brain is involved, the capacity of a drug to penetrate the blood brain barrier must be considered. Exogenous antioxidants of varying chemical structures have been investigated, but most of them poorly cross the blood brain barrier [85]. In this thesis, a standardized and extensive cell line panel has been developed for fast and large-scale screening of compounds (new chemical substances as well as known nutritional supplements) with therapeutic potential in OXPHOS disorders. Default parameters have been documented extensively to evaluate the effect of the candidate compounds reliably and in detail. Furthermore, monitoring the amount of oxidative (protein) damage and/or apoptosis have been pointed out as valuable markers in OXPHOS pathology and can possibly also be considered to assess the effect of compounds on disease outcome. 


\section{Therapeutic approaches}

Current therapy approaches are based on specific treatment of particular mitochondrial diseases, treatment of symptoms of mitochondrial disease, (prevention by) exercise therapy and inhibition of ROS damage and prevention of germline transmission as explained below. Specific treatments include CoQ10 replacement therapy for patients with CABC1 [38] or ETFDH [39] mutation, L-arginine supplementation in MELAS patients $[86,87]$ and peritoneal dialysis or allogeneic hematopoietic stem cell transplantation in patients with TYMP mutations and mitochondrial neurogastrointestinal encephalomyopathy [MNGIE] [88]. Furthermore, more than a decade ago, riboflavin supplementation has been shown to be beneficial for certain patients with complex I deficiency by Scholte et al [89], while recently the genetic basis for this finding (ACAD9 mutation) has been elucidated in these patients [59]. Although not proven, it is striking that untreated patients with ACAD9 mutations die at young age of cardiomyopathies [90, 91].

\section{Symptomatic therapy}

Current symptomatic pharmacological therapy includes, amongst others, sodium bicarbonate and dichloroacetate for lactic acidosis, anticonvulsants for epileptic seizures and beta blockers for cardiomyopathy [84, 92]. Despite that the effectiveness is not clear for most compounds, various treatment cocktails of vitamins and cofactors (e.g. riboflavin, thiamine, folic acid, L-carnitine, creatine monohydrate and idebenone) are administrated to patients with OXPHOS disease because they are presumed harmless [93]. Non-pharmacological treatment options include organ transplantation (e.g. kidney or heart with single organ involvement), corrective ptosis surgery, gastrostomy and deep brain stimulation [84].

\section{Prevention of transmission}

Instead of treating the disease itself, one might also choose for the prevention of $\mathrm{mtDNA}$ disease segregation in families. A number of approaches currently available are prenatal diagnosis using chorionic villus sampling, preimplantation genetic diagnosis (PGD), or nuclear transfer, which is still in the experimental phase [94]. Prenatal diagnosis (PND) is applicable for nuclear gene defects. For mtDNA disease this is not reliable, as the clinical severity of the phenotype after birth cannot reliably be predicted for most mtDNA mutations, based on the mutation percentage in chorionic villus [95]. At present, $P G D$ is the better alternative for carriers of heteroplasmic mtDNA mutations [95]. In PGD, embryos are generated by in vitro fertilization and tested for the mitochondrial DNA mutations. Only the embryos with low or undetectable amounts of mutant DNA are transferred to the uterus, which unlike PND, avoids the dilemma of pregnancy termination. Although it cannot guarantee that a child will be unaffected, PGD aims at reducing the risk of having an affected child [95]. Because of this suboptimal testing, a number of ethical questions have arisen on the selection of embryos, the setting of safe margins for mtDNA mutations and the wishes of the parents [96]. In the future, nuclear transfer might be promising. This involves the fusion of a nucleus from a fertilized oocyte with an enucleated donor oocyte. Although recent 
studies have shown the feasibility hereof in apes in vivo [97] and in humans in vitro [94, 98], the technique is still experimental, implies many unknowns (e.g. the effect on nucleo-mitochondrial interactions) and brings about even more ethical issues [99].

\section{Gene therapy}

In the last decade, a number of approaches in gene therapy, especially for long-term treatment of mtDNA related OXPHOS disorders, have been explored [93]. One strategy focuses on introducing a wild type copy of the mutated mitochondrial gene into the nucleus, translating the protein in the cytosol and importing normal copies of the protein into the mitochondria (allotopic expression). Although elegant, this method involves some obstacles to overcome. The protein has to be targeted to the mitochondria (by adding a targeting signal), imported into the mitochondria and assembled into a multisubunit complex. In addition, translation in the cytosol requires the use of the standard genetic code instead of the mitochondrial code [100]. Some successes have been reported with the NARP m.T8993G mutation in ATP6, the LHON mutations m.G3460A and m.G11778A in ND1 and ND4 respectively and the MERRF m.A8344G mutation in tRNALys [101-103]. Allotopic expression of the wild type genes in cybrid cell lines or patient fibroblasts carrying the (nearly, >95\%) homoplasmic mutations (partially) rescued the biochemical defect [101-103]. Another promising long-term therapy is to selectively inhibit replication of mutant mtDNA molecules (antigenomic therapy). The challenge is to find an agent that discriminates between wild type and mutant mtDNA, that can be imported into the mitochondria and binds the mutant mtDNA irreversibly to prevent its release when natural replication is occurring [100]. In vitro experiments with peptide nucleic acids showed their ability to selectively inhibit replication of the m.A8344G MERRF mutant mtDNA [104]. However, problems related with the import of the antigenomic molecules into the mitochondria still have to be resolved [100]. So far, these approaches have only been tested in vitro. Due to the current lack of pathogenic heteroplasmic mtDNA mouse models, further development and translation to human patients will not be for the near future [105].

Even though few OXPHOS disorders can be treated because of a specific metabolic defect inherent to a certain mutation, most cannot. The phenotypic diversity of patients with the same genetic defect and the genetic heterogeneity of patients with the same syndrome or symptoms emphasize that therapy is better not based on the patients' phenotype and/or genotype but on the molecular pathways, some of which are described in this thesis that are triggered or changed in the patients' tissues. Therefore, the future strategy would be to systematically monitor key pathways in tissues of OXPHOS patients, irrespective of their genetic or biochemical diagnosis, and from this build a personal therapy plan to optimize the treatment effect and reduce the amount of ineffective medication. 


\section{References}

1. Alberts B, J.A., Lewis J, Raff M, Roberts K, Walter P, Molecular Biology of the Cell, 4th edition. 2002, New York: Garland Science.

2. Gissi, C., F. lannelli, and G. Pesole, Evolution of the mitochondrial genome of Metazoa as exemplified by comparison of congeneric species. Heredity, 2008. 101(4): p. 301-20.

3. Croteau, D.L. and V.A. Bohr, Repair of oxidative damage to nuclear and mitochondrial DNA in mammalian cells. J Biol Chem, 1997. 272(41): p. 25409-12.

4. Wallace, D.C., A mitochondrial paradigm of metabolic and degenerative diseases, aging, and cancer: a dawn for evolutionary medicine. Annu Rev Genet, 2005. 39: p. 359-407.

5. Clayton, D.A., J.N. Doda, and E.C. Friedberg, The absence of a pyrimidine dimer repair mechanism in mammalian mitochondria. Proc Natl Acad Sci U S A, 1974. 71(7): p. 2777-81.

6. Takao, M., et al., Mitochondrial targeting of human DNA glycosylases for repair of oxidative DNA damage. Nucleic Acids Res, 1998. 26(12): p. 2917-22.

7. Pinz, K.G. and D.F. Bogenhagen, Efficient repair of abasic sites in DNA by mitochondrial enzymes. Mol Cell Biol, 1998. 18(3): p. 1257-65.

8. Seo, A.Y., et al., New insights into the role of mitochondria in aging: mitochondrial dynamics and more. J Cell Sci, 2010. 123(Pt 15): p. 2533-42.

9. Neiman, M. and D.R. Taylor, The causes of mutation accumulation in mitochondrial genomes. Proc Biol Sci, 2009. 276(1660): p. 1201-9.

10. Jansen, R.P., Germline passage of mitochondria: quantitative considerations and possible embryological sequelae. Hum Reprod, 2000. 15 Suppl 2: p. 112-28.

11. Balloux, F., et al., Climate shaped the worldwide distribution of human mitochondrial DNA sequence variation. Proc Biol Sci, 2009. 276(1672): p. 3447-55.

12. Mishmar, D., et al., Natural selection shaped regional mtDNA variation in humans. Proc Natl Acad Sci U S A, 2003. 100(1): p. 171-6.

13. Wallace, D.C., Bioenergetics and the epigenome: interface between the environment and genes in common diseases. Dev Disabil Res Rev, 2010. 16(2): p. 114-9.

14. Mishmar, D. and I. Zhidkov, Evolution and disease converge in the mitochondrion. Biochim Biophys Acta, 2010. 1797(6-7): p. 1099-104.

15. Stewart, J.B., et al., Strong purifying selection in transmission of mammalian mitochondrial DNA. PLoS Biol, 2008. 6(1): p. e10.

16. Trifunovic, A., et al., Premature ageing in mice expressing defective mitochondrial DNA polymerase. Nature, 2004. 429(6990): p. 417-23.

17. Vazquez-Memije, M.E., et al., Cellular and functional analysis of four mutations located in the mitochondrial ATPase6 gene. J Cell Biochem, 2009. 106(5): p. 878-86.

18. Trounce, I.A. and C.A. Pinkert, Cybrid models of mtDNA disease and transmission, from cells to mice. Curr Top Dev Biol, 2007. 77: p. 157-83.

19. Smits, P., et al., Functional consequences of mitochondrial tRNA Trp and tRNA Arg mutations causing combined OXPHOS defects. Eur J Hum Genet, 2010. 18(3): p. 324-9.

20. van der Westhuizen, F.H., et al., Human mitochondrial complex I deficiency: investigating transcriptional responses by microarray. Neuropediatrics, 2003. 34(1): p. 14-22.

21. Valsecchi, F., et al., Complex I disorders: causes, mechanisms, and development of treatment strategies at the cellular level. Dev Disabil Res Rev, 2010. 16(2): p. 17582.

22. van den Heuvel, L.P., J.A. Smeitink, and R.J. Rodenburg, Biochemical examination of fibroblasts in the diagnosis and research of oxidative phosphorylation (OXPHOS) defects. Mitochondrion, 2004. 4(5-6): p. 395-401. 
23. Moran, M., et al., Mitochondrial bioenergetics and dynamics interplay in complex Ideficient fibroblasts. Biochim Biophys Acta, 2010. 1802(5): p. 443-53.

24. Eagle, H., A.E. Freeman, and M. Levy, The amino acid requirements of monkey kidney cells in first culture passage. J Exp Med, 1958. 107(5): p. 643-52.

25. Rossignol, R., et al., Energy substrate modulates mitochondrial structure and oxidative capacity in cancer cells. Cancer Res, 2004. 64(3): p. 985-93.

26. Wolfrom, C., et al., Glutamine dependency of human skin fibroblasts: modulation by hexoses. Exp Cell Res, 1989. 183(2): p. 303-18.

27. Koopman, W.J., et al., Computer-assisted live cell analysis of mitochondrial membrane potential, morphology and calcium handling. Methods, 2008. 46(4): $\mathrm{p}$. 304-11.

28. Willems, P.H., et al., Mitochondrial Ca2+ homeostasis in human NADH:ubiquinone oxidoreductase deficiency. Cell Calcium, 2008. 44(1): p. 123-33.

29. Valsecchi, F., et al., Calcium and ATP handling in human NADH:ubiquinone oxidoreductase deficiency. Biochim Biophys Acta, 2009. 1792(12): p. 1130-7.

30. Kujoth, G.C., et al., Mitochondrial DNA mutations, oxidative stress, and apoptosis in mammalian aging. Science, 2005. 309(5733): p. 481-4.

31. Kruse, S.E., et al., Mice with mitochondrial complex I deficiency develop a fatal encephalomyopathy. Cell Metab, 2008. 7(4): p. 312-20.

32. Ingraham, C.A., et al., NDUFS4: creation of a mouse model mimicking a Complex I disorder. Mitochondrion, 2009. 9(3): p. 204-10.

33. Saneto, R.P. and R.K. Naviaux, Polymerase gamma disease through the ages. Dev Disabil Res Rev, 2010. 16(2): p. 163-74.

34. Tuppen, H.A., et al., Mitochondrial DNA mutations and human disease. Biochim Biophys Acta, 2010. 1797(2): p. 113-28.

35. Plasterk, R.H., Hershey heaven and Caenorhabditis elegans. Nat Genet, 1999. 21(1): p. 63-4.

36. de Vries, M.C., et al., Normal biochemical analysis of the oxidative phosphorylation (OXPHOS) system in a child with POLG mutations: A cautionary note. J Inherit Metab Dis, 2008.

37. Thorburn, D.R., et al., Biochemical and molecular diagnosis of mitochondrial respiratory chain disorders. Biochim Biophys Acta, 2004. 1659(2-3): p. 121-8.

38. Gerards, M., et al., Nonsense mutations in CABC1/ADCK3 cause progressive cerebellar ataxia and atrophy. Mitochondrion, 2010. 10(5): p. 510-5.

39. Gempel, K., et al., The myopathic form of coenzyme Q10 deficiency is caused by mutations in the electron-transferring-flavoprotein dehydrogenase (ETFDH) gene. Brain, 2007. 130(Pt 8): p. 2037-44.

40. Smeitink, J.A., et al., Mitochondrial medicine: a metabolic perspective on the pathology of oxidative phosphorylation disorders. Cell Metab, 2006. 3(1): p. 9-13.

41. Robinson, B.H., Lactic acidemia and mitochondrial disease. Mol Genet Metab, 2006. 89(1-2): p. 3-13.

42. Smeitink, J.A., Mitochondrial disorders: clinical presentation and diagnostic dilemmas. J Inherit Metab Dis, 2003. 26(2-3): p. 199-207.

43. Esteitie, N., et al., Secondary metabolic effects in complex I deficiency. Ann Neurol, 2005. 58(4): p. 544-52.

44. Reinecke, F., J.A. Smeitink, and F.H. van der Westhuizen, OXPHOS gene expression and control in mitochondrial disorders. Biochim Biophys Acta, 2009. 1792(12): p. 1113-21.

45. Berridge, M.J., M.D. Bootman, and H.L. Roderick, Calcium signalling: dynamics, homeostasis and remodelling. Nat Rev Mol Cell Biol, 2003. 4(7): p. 517-29.

46. Brini, M., et al., A calcium signaling defect in the pathogenesis of a mitochondrial DNA inherited oxidative phosphorylation deficiency. Nat Med, 1999. 5(8): p. 951-4. 
47. Visch, H.J., et al., Decreased agonist-stimulated mitochondrial ATP production caused by a pathological reduction in endoplasmic reticulum calcium content in human complex I deficiency. Biochim Biophys Acta, 2006. 1762(1): p. 115-23.

48. Wojewoda, M., et al., Effect of selenite on basic mitochondrial function in human osteosarcoma cells with chronic mitochondrial stress. Mitochondrion, 2011.

49. Kaminishi, T. and K.J. Kako, Sensitivity to oxidants of mitochondrial and sarcoplasmic reticular calcium uptake in saponin-treated cardiac myocytes. Basic Res Cardiol, 1989. 84(3): p. 282-90.

50. Brookes, P.S., et al., Calcium, ATP, and ROS: a mitochondrial love-hate triangle. Am J Physiol Cell Physiol, 2004. 287(4): p. C817-33.

51. De Stefani, D., et al., $A$ forty-kilodalton protein of the inner membrane is the mitochondrial calcium uniporter. Nature, 2011. 476(7360): p. 336-40.

52. Baughman, J.M., et al., Integrative genomics identifies MCU as an essential component of the mitochondrial calcium uniporter. Nature, 2011. 476(7360): p. 341-5.

53. James, A.M., et al., Altered mitochondrial function in fibroblasts containing MELAS or MERRF mitochondrial DNA mutations. Biochem J, 1996. 318 ( Pt 2): p. 401-7.

54. Distelmaier, F., et al., Mitochondrial complex I deficiency: from organelle dysfunction to clinical disease. Brain, 2009. 132(Pt 4): p. 833-42.

55. Iglewski, M., et al., Mitochondrial fission and autophagy in the normal and diseased heart. Curr Hypertens Rep, 2010. 12(6): p. 418-25.

56. Twig, G. and O.S. Shirihai, The interplay between mitochondrial dynamics and mitophagy. Antioxid Redox Signal, 2011. 14(10): p. 1939-51.

57. Chen, C.Y., et al., Decreased heat shock protein 27 expression and altered autophagy in human cells harboring A8344G mitochondrial DNA mutation. Mitochondrion, 2011. 11(5): p. 739-49.

58. Alemi, M., et al., Mitochondrial DNA deletions inhibit proteasomal activity and stimulate an autophagic transcript. Free Radic Biol Med, 2007. 42(1): p. 32-43.

59. Gerards, M., et al., Riboflavin-responsive oxidative phosphorylation complex I deficiency caused by defective ACAD9: new function for an old gene. Brain, 2011. 134(Pt 1): p. 210-9.

60. Selivanov, V.A., et al., Reactive oxygen species production by forward and reverse electron fluxes in the mitochondrial respiratory chain. PLoS Comput Biol, 2011. 7(3): p. e1001115.

61. Verkaart, S., et al., Superoxide production is inversely related to complex I activity in inherited complex I deficiency. Biochim Biophys Acta, 2007. 1772(3): p. 373-81.

62. Huttemann, M., et al., Regulation of oxidative phosphorylation, the mitochondrial membrane potential, and their role in human disease. J Bioenerg Biomembr, 2008. 40(5): p. 445-56.

63. Korshunov, S.S., V.P. Skulachev, and A.A. Starkov, High protonic potential actuates a mechanism of production of reactive oxygen species in mitochondria. FEBS Lett, 1997. 416(1): p. 15-8.

64. Munnich, A. and P. Rustin, Clinical spectrum and diagnosis of mitochondrial disorders. Am J Med Genet, 2001. 106(1): p. 4-17.

65. Tretter, L. and V. Adam-Vizi, Generation of reactive oxygen species in the reaction catalyzed by alpha-ketoglutarate dehydrogenase. J Neurosci, 2004. 24(36): p. 77718.

66. Starkov, A.A., et al., Mitochondrial alpha-ketoglutarate dehydrogenase complex generates reactive oxygen species. J Neurosci, 2004. 24(36): p. 7779-88.

67. Bedard, K. and K.H. Krause, The NOX family of ROS-generating NADPH oxidases: physiology and pathophysiology. Physiol Rev, 2007. 87(1): p. 245-313.

68. Pendyala, S. and V. Natarajan, Redox regulation of Nox proteins. Respir Physiol Neurobiol, 2010. 174(3): p. 265-71.

69. Mancuso, M., et al., Oxidative stress biomarkers in mitochondrial myopathies, basally and after cysteine donor supplementation. J Neurol, 2010. 257(5): p. 774-81. 
70. Bjelakovic, G., et al., Mortality in randomized trials of antioxidant supplements for primary and secondary prevention: systematic review and meta-analysis. JAMA, 2007. 297(8): p. 842-57.

71. Tkachev, V.O., E.B. Menshchikova, and N.K. Zenkov, Mechanism of the Nrf2/Keap1/ARE signaling system. Biochemistry (Mosc), 2011. 76(4): p. 407-22.

72. Dassa, E.P., et al., The mtDNA NARP mutation activates the actin-Nrf2 signaling of antioxidant defenses. Biochem Biophys Res Commun, 2008. 368(3): p. 620-4.

73. Alfieri, A., et al., Targeting the Nrf2-Keap1 antioxidant defence pathway for neurovascular protection in stroke. J Physiol, 2011.

74. Barone, M.C., G.P. Sykiotis, and D. Bohmann, Genetic activation of Nrf2 signaling is sufficient to ameliorate neurodegenerative phenotypes in a Drosophila model of Parkinson's disease. Dis Model Mech, 2011.

75. Muscarella, L.A., et al., Frequent epigenetics inactivation of KEAP1 gene in nonsmall cell lung cancer. Epigenetics, 2011. 6(6): p. 710-9.

76. Reeves, M.A., F.P. Bellinger, and M.J. Berry, The neuroprotective functions of selenoprotein $M$ and its role in cytosolic calcium regulation. Antioxid Redox Signal, 2010. 12(7): p. 809-18.

77. Jurynec, M.J., et al., Selenoprotein $N$ is required for ryanodine receptor calcium release channel activity in human and zebrafish muscle. Proc Natl Acad Sci U S A, 2008. 105(34): p. 12485-90.

78. Verma, S., et al., Selenoprotein $K$ knockout mice exhibit deficient calcium flux in immune cells and impaired immune responses. J Immunol, 2011. 186(4): p. 2127-37.

79. Muller, M., et al., Nrf2 target genes are induced under marginal selenium-deficiency. Genes Nutr, 2010. 5(4): p. 297-307.

80. Kerr, J.F., A.H. Wyllie, and A.R. Currie, Apoptosis: a basic biological phenomenon with wide-ranging implications in tissue kinetics. Br J Cancer, 1972. 26(4): p. 239-57.

81. Aure, K., et al., Apoptosis in mitochondrial myopathies is linked to mitochondrial proliferation. Brain, 2006. 129(Pt 5): p. 1249-59.

82. Dimauro, S. and P. Rustin, A critical approach to the therapy of mitochondrial respiratory chain and oxidative phosphorylation diseases. Biochim Biophys Acta, 2009. 1792(12): p. 1159-67.

83. Zeviani, M., Train, train, train! No pain, just gain. Brain, 2008. 131(Pt 11): p. 2809-11.

84. McFarland, R., R.W. Taylor, and D.M. Turnbull, A neurological perspective on mitochondrial disease. Lancet Neurol, 2010. 9(8): p. 829-40.

85. Gilgun-Sherki, Y., E. Melamed, and D. Offen, Oxidative stress inducedneurodegenerative diseases: the need for antioxidants that penetrate the blood brain barrier. Neuropharmacology, 2001. 40(8): p. 959-75.

86. Lekoubou, A., et al., Effect of long-term oral treatment with L-arginine and idebenone on the prevention of stroke-like episodes in an adult MELAS patient. Rev Neurol (Paris), 2011. 167(11): p. 852-5.

87. Koga, Y., et al., Molecular pathology of MELAS and l-arginine effects. Biochim Biophys Acta, 2011.

88. Halter, J., et al., Allogeneic hematopoietic SCT as treatment option for patients with mitochondrial neurogastrointestinal encephalomyopathy (MNGIE): a consensus conference proposal for a standardized approach. Bone Marrow Transplant, 2011. 46(3): p. 330-7.

89. Scholte, H.R., et al., Riboflavin-responsive complex I deficiency. Biochim Biophys Acta, 1995. 1271(1): p. 75-83.

90. Haack, T.B., et al., Exome sequencing identifies ACAD9 mutations as a cause of complex I deficiency. Nat Genet, 2010. 42(12): p. 1131-4.

91. Nouws, J., et al., Acyl-CoA dehydrogenase 9 is required for the biogenesis of oxidative phosphorylation complex I. Cell Metab, 2010. 12(3): p. 283-94.

92. Chinnery, P., et al., Treatment for mitochondrial disorders. Cochrane Database Syst Rev, 2006(1): p. CD004426. 
93. Horvath, R., G. Gorman, and P.F. Chinnery, How can we treat mitochondrial encephalomyopathies? Approaches to therapy. Neurotherapeutics, 2008. 5(4): p. 558-68.

94. Craven, L., et al., Pronuclear transfer in human embryos to prevent transmission of mitochondrial DNA disease. Nature, 2010. 465(7294): p. 82-5.

95. Poulton, J., et al., Preventing transmission of maternally inherited mitochondrial DNA diseases. BMJ, 2009. 338: p. b94.

96. Jacobs, L.J., et al., The transmission of OXPHOS disease and methods to prevent this. Hum Reprod Update, 2006. 12(2): p. 119-36.

97. Tachibana, M., et al., Mitochondrial gene replacement in primate offspring and embryonic stem cells. Nature, 2009. 461(7262): p. 367-72.

98. Heindryckx, B., et al., Embryo development after successful somatic cell nuclear transfer to in vitro matured human germinal vesicle oocytes. Hum Reprod, 2007. 22(7): p. 1982-90.

99. Bredenoord, A.L., G. Pennings, and G. de Wert, Ooplasmic and nuclear transfer to prevent mitochondrial DNA disorders: conceptual and normative issues. Hum Reprod Update, 2008. 14(6): p. 669-78.

100. Smith, P.M., et al., Strategies for treating disorders of the mitochondrial genome. Biochim Biophys Acta, 2004. 1659(2-3): p. 232-9.

101. Manfredi, G., et al., Rescue of a deficiency in ATP synthesis by transfer of MTATP6, a mitochondrial DNA-encoded gene, to the nucleus. Nat Genet, 2002. 30(4): p. 3949.

102. Bonnet, C., et al., The optimized allotopic expression of ND1 or ND4 genes restores respiratory chain complex I activity in fibroblasts harboring mutations in these genes. Biochim Biophys Acta, 2008. 1783(10): p. 1707-17.

103. Kolesnikova, O.A., et al., Nuclear DNA-encoded tRNAs targeted into mitochondria can rescue a mitochondrial DNA mutation associated with the MERRF syndrome in cultured human cells. Hum Mol Genet, 2004. 13(20): p. 2519-34.

104. Taylor, R.W., et al., Selective inhibition of mutant human mitochondrial DNA replication in vitro by peptide nucleic acids. Nat Genet, 1997. 15(2): p. 212-5.

105. Smith, P.M. and R.N. Lightowlers, Altering the balance between healthy and mutated mitochondrial DNA. J Inherit Metab Dis, 2011. 34(2): p. 309-13. 
Summary 
Mitochondrial disorders are often fatal multisystem disorders or syndromes, associated with abnormalities of the terminal component of mitochondrial energy metabolism, i.e. oxidative phosphorylation (OXPHOS). These diseases mainly manifest in tissues with high energy demands such as the heart, brain, liver and muscle and are further referred to as OXPHOS disorders. It is estimated that OXPHOS disorders affect about 1 in 5000 individuals. The OXPHOS system is responsible for cellular aerobic energy production and its subunits are encoded by over 80 genes of which 37 are located in the mitochondrial DNA (mtDNA). However, more than 1000 nuclear DNA (nDNA) genes are involved OXPHOS function, assembly and maintenance. Accordingly, mutations in the mtDNA or nDNA genes encoding structural OXPHOS elements or nDNA encoded factors involved in the maintenance or assembly often lead to OXPHOS disorders. The extreme genetic and phenotypic heterogeneity and the lack of consistent genotypephenotype correlations complicate genetic testing of these disorders. Moreover, the underlying pathological mechanisms of these diseases are only partly understood and there is a lack of efficient therapies. The aim of this thesis is to create and evaluate models and approaches to facilitate diagnostics of mtDNA-based OXPHOS disorders, study OXPHOS pathophysiology and as a consequence identify new potential targets for therapeutic interventions. In that respect, mitochondrial DNA resequencing chips (chapter 2), microarray gene expression profiling (chapter 3 and 4) and a set of oxidative stress markers (chapter 5) have been applied to obtain new information on the distribution of mtDNA variation in the general population and pathological and/or adaptive processes involved in OXPHOS disease, respectively. Chapter 6 discusses the validity and relevance of the different models for the study of OXPHOS disorders.

Evaluating pathogenicity of new unclassified variants identified in patients with symptoms related to OXPHOS disorders is not straightforward. Improved insight in the presence, tolerance, negative selection and consequences of mtDNA variation in the human population will give valuable information for the diagnostic classification of these mtDNA variants. In chapter 2 , the mtDNA of 730 subjects was resequenced and compared with the revised reference sequence. The locations of non-pathogenic variants identified in this study and known pathogenic mtDNA mutations were evaluated in terms of functional importance and conservation. For the non-pathogenic variation, there was a preferential selection against variants in protein coding genes with high sequence conservation. Also for tRNA genes, neutral variants and pathogenic mutations showed differences in functional location (loop or stem) and sequence or base pair conservation. Thus, evaluating the conservation and functional importance of a newly identified variant remain the most important parameters. It can be expected that this approach combined with the data generated by next generation sequencing will lead to a detailed map of all variants ever detected, both for the mitochondrial and nuclear DNA.

When a genetic diagnosis for an OXPHOS disorder is established, the pathogenic process has to be unraveled in order to determine cause and mechanism of the disease symptoms for proper prognosis and to explore potential therapeutic strategies. For pathophysiological studies, affected tissues from patients would allow the most 
relevant and specific disease processes to be identified. However, for diseases affecting non-dividing tissues (e.g. brain), the availability and amount of material is obviously limited. Therefore, model systems such as other patient derived tissues (e.g. muscle biopsies, fibroblasts) can be used. Even though these tissues might be less or not affected in OXPHOS patients, they can still provide clues for pathological processes in other affected tissues.

To identify new biomarkers or potential therapeutic targets, whole genome gene expression profiling and pathway analysis have been applied to explore the underlying molecular processes in patients with POLG1 mutations and patients with complex I $(\mathrm{Cl})$ deficiency, the largest patient groups with mitochondrial disease. DNA polymerase gamma (pol $\mathrm{Y}$; POLG1 gene) is the only DNA polymerase involved in maintenance of the mtDNA. POLG1 mutations have been reported to cause a broad variety of phenotypes involving hepatopathy usually early in life, isolated myopathy in older individuals or brain abnormalities at any age. The correlation between genotype and phenotype in patients with POLG1 mutations is sometimes tight but more often variable. In chapter 3 , skeletal muscle from patients with mutations in the POLG1 gene was used as a model for pathological changes in liver and brain. The majority of these patients exhibited subtle morphological skeletal muscle changes and gene expression analysis mainly pointed to an energy metabolism switch (decreased beta oxidation, increased coupling of electron transfer and ATP production) and hinted towards changes in oxidative stress and apoptosis. The latter increased processes were further validated in fibroblast cultures, liver and brain and were shown to be increased in part of the patients. Ultimately, this study revealed the involvement of these processes in the pathology of at least part of human POLG1 patients for the first time and emphasizes the previously described heterogeneity in this patient group, requesting an individual-based approach. In chapter 4, primary fibroblast cultures of $\mathrm{Cl}$ deficient patients with a nuclear mutation were used as a model to study pathology. $\mathrm{Cl}$ deficiency is the most frequently encountered defect in mitochondrial energy metabolism and is associated with e.g. Leigh and LHON disease, fatal infantile acidosis, neonatal cardiomyopathy with lactic acidosis, leucodystrophy with macrocephaly and hepatopathy with renal tubulopathy. Although children usually have a normal prenatal development, symptoms start occurring during their first year of life after which the disease deteriorates rapidly and may become fatal. Because fibroblasts primarily ( 70\%) produce ATP by glycolysis, fibroblasts were experimentally stimulated to use OXPHOS (and express the defect) by culturing them without glucose in the presence of galactose. Whole genome expression profiling showed that patient fibroblasts responded to oxidative stress by Nrf2-mediated induction of the glutathione antioxidant system and Gadd45-mediated activation of the DNA damage response pathway. Furthermore, the observed reduced expression of selenoproteins could explain the disturbed calcium homeostasis previously described for the patient fibroblasts and might be linked to endoplasmic reticulum stress. These results indicate that both glutathione and selenium metabolism are potentially therapeutic targets in $\mathrm{Cl}$ deficiency. 
Oxidative stress was observed in both the POLG1 and Cl deficiency models (chapter 3 and 4) and has been implicated to play a role in OXPHOS disorders in literature. However, most studies did not report on the full spectrum of ROS levels, ROS detoxification (antioxidant status) and oxidative damage. Nevertheless, OXPHOS patients are often supplemented with antioxidants, even though their efficacy is highly anecdotic. Therefore, a pipe-line for the characterization of ROS levels, stress induced (antioxidant) gene expression and oxidative protein damage in fibroblasts was established (chapter 5). Using this approach, the level of oxidative stress in fibroblasts of three different patient groups (POLG1 patients and patients with mtDNA tRNA-Leu or $\mathrm{Cl}$ mutations) was compared. Whereas almost all cell lines showed increased ROS levels compared with wild type fibroblasts, only few concomitantly exhibited increased oxidative protein damage. The absence of oxidative damage in the other cell lines could partially be explained by increased stress-related (antioxidant) gene expression. Even though fibroblasts are better able to adapt to a genetic or biochemical defect than severely affected post-mitotic tissues, the current study demonstrated the ability to measure altered ROS levels and oxidative damage in these cells. Therefore, the fibroblast model provides an indication for the oxidative status of a patient and information on which adaptive processes are therapeutically relevant. The characterized fibroblasts models in chapter $\mathbf{4}$ and $\mathbf{5}$ can also be used for highthroughput screening of potentially beneficial compounds using the same read-out parameter, as used for establishing the pathophysiological processes.

In this thesis, models and methodologies have been developed for the improvement of diagnostics, prognostics, understanding and therapy of specific OXPHOS disorders, for which the genetic defect was known. Comparable models can be established for other OXPHOS disorders as well, applying the same methodologies. However, as discussed in chapter 6, no single model system can cover every aspect from the identification of pathological processes to the screening of therapeutic compounds. Our in vitro data based on patient material should be validated and followed-up in more advanced (animal) models before eventually translating them into patient care. 

Samenvatting 
Mitochondriële afwijkingen zijn vaak dodelijke afwijkingen of syndromen in meerdere organen die geassocieerd zijn met abnormaliteiten van de laatste component van het mitochondriële energiemetabolisme, oxidatieve fosforylering (OXPHOS). Deze ziekten treffen voornamelijk weefsels met een hoge energiebehoefte zoals het hart, de hersenen, de lever en spier. Dezen zullen verder OXPHOS ziekten genoemd worden. Naar schatting wordt 1 op 5000 individuen getroffen door een OXPHOS ziekte. Het OXPHOS systeem is verantwoordelijk voor de cellulaire aërobe energie productie en de afzonderlijke subeenheden worden door meer dan 80 genen gecodeerd waarvan er 37 gelegen zijn in het mitochondrieel DNA (mtDNA). Er zijn echter meer dan 1000 genen in het nucleaire DNA (nDNA) betrokken bij OXPHOS functie, assemblage en onderhoud. Daarom leiden mutaties in mtDNA of nDNA genen die coderen voor structurele OXPHOS elementen of in nDNA gecodeerde factoren betrokken bij OXPHOS onderhoud en de assemblage vaak tot OXPHOS ziekten. De extreme genetische en fenotypische heterogeniteit en het gebrek aan consistente genotypefenotype correlaties maken het genetische testen van deze ziekten complex. Verder wordt het onderliggende pathologische mechanisme slechts gedeeltelijk begrepen en is er een gebrek aan efficiënte therapieën. Het doel van deze thesis is de creatie en evaluatie van modellen en strategieën om de diagnostiek van mtDNA-gebaseerde OXPHOS ziekten makkelijker te maken, de pathofysiologie van OXHPOS ziekten te bestuderen en bijgevolg nieuwe potentiële doelen voor therapie te identificeren. Daarom werden mtDNA resequencing chips (hoofdstuk 2), microarray genexpressie analyse (hoofdstuk 3 en 4) en een set van oxidatieve stress merkers (hoofdstuk 5) ingezet om nieuwe informatie te verkrijgen over de verdeling van mtDNA variatie in de normale populatie en over de pathologische en/of adaptieve processen betrokken bij OXPHOS ziekten. Hoofdstuk 6 bediscussieert de validiteit en de relevantie van de verschillende modellen voor het bestuderen van OXPHOS ziekten.

De evaluatie van de pathogeniciteit van nieuwe ongeclassificeerde varianten in patiënten met symptomen gerelateerd aan OXPHOS ziekten is niet eenvoudig. Een verbeterd inzicht in de aanwezigheid van, de tolerantie van, negatieve selectie tegen en de gevolgen van mtDNA variatie in de menselijke populatie zal waardevolle informatie verschaffen voor de diagnostische classificatie van deze mtDNA varianten. In hoofdstuk 2 werd het mtDNA van 730 individuen geanalyseerd en vergeleken met de gereviseerde referentiesequentie. De locatie van niet-pathogene varianten die geïdentificeerd werden in deze studie en bekende pathogene mutaties werd geëvalueerd met betrekking tot functioneel belang en conservering. Voor de nietpathogene variatie was er een preferentiële selectie tegen varianten in eiwitcoderende genen met een sterke conservering. Ook voor de tRNA genen toonden de neutrale varianten en pathogene mutaties verschillen in functionele locatie (loop of stam) en sequentie of basenpaar conservering. Dus, de evaluatie van de conservering en het functionele effect van een nieuw geïdentificeerde variant blijven de meest belangrijke parameters. Verwacht wordt dat deze aanpak, in combinatie met de data van next generation seqeuncing, zal leiden tot een gedetailleerde kaart van alle varianten die ooit gedetecteerd werden, zowel voor het mitochondriële als het nucleaire DNA. 
Wanneer er een genetische diagnose voor een OXPHOS ziekte is, moet het pathogene process nog ontrafeld worden om de oorzaak en het mechanisme dat leidt tot de symptomen te bepalen zodat de juiste prognose en het onderzoeken van mogelijke therapieën mogelijk is. Voor pathofysiologische studies, zouden de aangedane weefsels van patiënten toelaten de meest relevante en specifieke processen te identificeren. Echter, voor aandoeningen van niet-delende weefsels (bijvoorbeeld hersenen) is de beschikbaarheid en hoeveelheid van materiaal beperkt. Daarom kunnen modelsystemen zoals andere weefsels (bijvoorbeeld spierbiopsies, fibroblasten) van de patiënten gebruikt worden. Hoewel deze weefsels minder of niet aangedaan kunnen zijn in OXPHOS patiënten, kunnen ze nog steeds aanwijzingen voor pathologische processen in andere aangedane weefsels opleveren.

Genexpressie-analyse van het volledige genoom en process-gebaseerde analyse werden toegepast om de onderliggende moleculaire processen in patiënten met POLG1 mutaties en patiënten met complex I $(\mathrm{Cl})$ deficiëntie te bestuderen en mogelijk nieuwe biomerkers of potentiële therapeutische doelwitten te identificeren. DNA polymerase gamma (pol y; POLG1 gen) is het enige DNA polymerase dat betrokken is bij het onderhoud van het mtDNA. POLG1 mutaties veroorzaken een brede waaier aan fenotypes inclusief hepatopathie op jonge leeftijd, geïsoleerde myopathie in oudere individuen en hersenafwijkingen op elke leeftijd. De correlatie tussen genotype en fenotype in patiënten met POLG1 mutaties is soms eenduidig maar meestal variabel. In hoofdstuk 3 werd skeletspier van patiënten met mutaties in het POLG1 gen gebruikt als model voor pathologische veranderingen in lever en hersenen. De meerderheid van deze patiënten hadden subtiele morfologische skeletspier veranderingen. Genexpressie-analyse wees voornamelijk op een omschakeling van het energie metabolisme (vermindere beta oxidatie, versterkte koppeling van elektronentransport en ATP productie) en suggereerde veranderingen in oxidatieve stress en apoptose. Deze laatste processen werden verder gevalideerd in fibroblastkweken, lever en hersenen en bleken verhoogd te zijn in een gedeelte van de patiënten. Uiteindelijk heeft deze studie voor het eerst aangetoond dat deze processen betrokken zijn bij de pathologie van minstens een gedeelte van de humane POLG1 patiënten. Voorts wordt de eerder beschreven heterogeniteit van deze patiëntengroep en de nood aan een individuele aanpak nogmaals benadrukt.

In hoofdstuk 4 werden primaire fibroblast kweken van $\mathrm{Cl}$ deficiënte patiënten met een nucleaire mutatie gebruikt als model om $\mathrm{Cl}$ pathologie te bestuderen. $\mathrm{Cl}$ deficiëntie is het meeste frequente defect in mitochondrieel energie metabolisme en is geassocieerd met bvb. Leigh en LHON ziekte, fatale infantiele acidose, neonatale cardiomyopathie met lactaat acidose, leukodystrofie met macrocefalie en hepatopathie met renale tubulopathie. Hoewel kinderen meestal een normale prenate ontwikkeling doormaken, beginnen de eerste symptomen te verschijnen tijdens hun eerste levensjaar waarna de ziekte snel verergert en tot overlijden kan leiden. Omdat fibroblasten voornamelijk $(\sim 70 \%)$ ATP produceren via glycolyse, werden de fibroblasten experimenteel gestimuleerd om OXPHOS te gebuiken (en het defect tot expressie te brengen) door hen te kweken zonder glucose in aanwezigheid van galactose. Genexpressie-analyse van het volledige genoom toonde aan dat patiëntfibroblasten op oxidatieve stress 
reageerden door Nrf2-gemedieerde inductie van het glutathion antioxidant systeem en Gadd45-gemedieerde activatie van de DNA schade respons. Verder kan de geobserveerde verminderde expressie van seleno-eiwitten de eerder beschreven verstoorde calcium homeostase in deze patiënten fibroblasten verklaren en kan dit ook gelinkt zijn aan endoplasmatisch reticulum stress. Deze resultaten tonen aan dat zowel glutathion als selenium metabolisme potentiële therapeutische kandidaten zijn bij $\mathrm{Cl}$ deficiëntie.

Oxidatieve stress werd in beide (POLG1 en $\mathrm{Cl}$ deficiëntie) modellen geobserveerd en wordt ook in de literatuur genoemd als een speler in OXPHOS ziekten. De meeste studies rapporteerden echter niet over het volledige spectrum van reactieve zuurstof soorten (ROS) niveaus, ROS detoxificatie (antioxidant status) en oxidatieve schade. Desalniettemin krijgen OXPHOS patiënten vaak antioxidant supplementen, ook al is hun efficiëntie sterk anecdotisch. Daarom werd een pijplijn opgezet voor de characterisatie van ROS niveaus, stress geïnduceerde (antioxidant) genexpressie en oxidatieve eiwit schade in fibroblasten (hoofdstuk 5). Met deze aanpak werd de mate van oxidatieve stress in fibroblasten van drie verschillende patiënten groepen (POLG1 patiënten en patiënten met mtDNA tRNA-Leu of Cl mutaties) vergeleken. Hoewel bijna alle cellijnen verhoogde ROS niveaus vertoonden vergeleken met controle fibroblasten, waren er slechts enkele met verhoogde oxidatieve eiwitschade. De afwezigheid van oxidatieve schade in de andere cellijnen kon gedeeltelijk verklaard worden door verhoogde stress-gerelateerde (antioxidant) genexpressie. Ook al zijn fibroblasten beter in staat om zich aan te passen aan een genetisch of biochemisch defect dan ernstig aangedane post-mitotische weefsel, de huidige studie laat zien dat het mogelijk is verhoogde ROS niveaus en oxidatieve schade in de cellen te meten. Daarom geeft het fibroblast model een indicatie voor de oxidatieve status van een patiënt en tevens ook informatie over welke adaptieve processen mogelijk van therapeutisch belang kunnen zijn. De gekarakteriseerde fibroblastmodellen in hoofdstuk 4 en 5 kunnen voorts ook gebruikt worden voor het high throughput screenen van potentiële therapeutische stoffen, gebruik makend van dezelfde uitleesparameters als voor de studie van de pathofysiologische processen.

In deze thesis werden modellen en methods ontwikkeld voor de verbetering van diagnose, prognose, het begrip en de therapie van specifieke OXPHOS ziekten waarvoor het genetisch defect gekend was. Vergelijkbare modellen kunnen ook opgezet worden andere OXPHOS ziekten. Echter, zoals aangehaald in hoofdstuk 6, zal geen enkel modelsysteem in staat zijn om elk aspect, van de identificatie van de pathologische processen tot de screening van therapeutische stoffen, te omvatten. Onze in vitro data, gebaseerd op patiëntenmateriaal, moet daarom ook gevalideerd en opgevolgd worden in meer geavanceerde (dier)modellen voordat ze vertaald kan worden naar patiëntenzorg. 

Dankwoord 
Is het dan eindelijk zover? Ik kan het bijna niet geloven... mijn boekje is klaar! En dat na 5 jaar die parodoxaal soms traag en soms snel leken om te vliegen. Natuurlijk werd ik gedurende deze tijd bijgestaan, geholpen, vermaakt... door een hele hoop mensen die ik hieronder wat uitgebreider wil bedanken. Ik zal me voor het grotendeels Nederlandse publiek alvast verontschuldigen voor de Vlaamse termen die hieronder wel eens de revue zouden kunnen passeren en andersom natuurlijk ook $\odot$.

Eerst en vooral wil ik Bert bedanken. Simpelweg, zonder jou zou dit boekje er niet zijn. Dank je dat je me de kans gegeven hebt om deze ervaringen op te doen. Als mijn promotor, en tevens enige begeleider, zorgde je ervoor dat ik de rode draad in het verhaal niet kwijt raakte. Op momenten dat ik wel eens minder gemotiveerd was, wist je toch weer de juiste dingen te zeggen. Van jou heb ik verder geleerd dat je in Nederland een mail begint met 'Beste' en eindigt met 'Groeten' (wat in jouw mails - zelfs héle korte - zelden tot nooit ontbreekt) in plaats van het Vlaamse 'Geachte' en 'Met vriendelijke groet'. Ik vond het ook heel fijn dat ik over alles open met je kon praten, zowel de leuke als de minder leuke dingen.

Volgende in rij is René. Dankjewel dat je mijn co-promotor wilde zijn. Ondanks dat Rotterdam en Maastricht ver van elkaar liggen, stond je altijd klaar om te helpen als dat mogelijk was. Ik vergeet nooit dat je ons (Auke, jij en ik) als 1 groep, met jou in Rotterdam en Auke en ik in Maastricht, voorstelde aan anderen tijdens een symposium in Nijmegen. Dat geeft wel aan dat de RoMa-mensen een clubje vormen van onderzoekers die graag samenwerken.

In Rotterdam zijn er nog meer mensen die ik graag zou willen bedanken: Kees, Rob en Wim. Kees, bedankt voor de vele OXPHOS activiteit metingen en dat je me hebt ingewijd in de wondere wereld van de biochemie. Rob, jou ken ik het kortst in Rotterdam, maar jouw bijdrage aan het POLG verhaal heeft het echt naar een hoger niveau gebracht. En Wim, jammer dat we elkaar al een tijd niet meer gezien hebben, maar dankjewel voor mijn eerste ervaring met ROS-kleuringen!

Naast Rotterdam en Maastricht heb ik nog een andere stad in Nederland ontdekt: Nijmegen. Jan, Be, Merei, Richard, Peter, Werner, John en alle anderen van het IOP Genomics project: dank jullie voor de leuke samenwerking! Ik heb echt genoten van de halfjaarlijkse projectmeetings en de discussies die daar plaatsvonden (ook al moesten we vanuit Maastricht soms wel héél vroeg vertrekken s'ochtends). Merei en Richard, ik denk dat het toch nog een mooi verhaal geworden is, niet? De moeite van de vele uren celkweek wel waard. Peter, Werner en John, bedankt voor het helpen met de ROSmetingen en de hartelijke ontvangst voor de paar weken die ik bij jullie in het lab heb doorgebracht.

Hoog tijd om even dichter bij huis te komen. De paranimfen: Florence, als voorganger heb ik veel van je geleerd, niet enkel wijsheden over het hele AlO-traject/gebeuren maar ook dagdagelijkse gebeurtenissen kwamen op de AlO-kamer vaak aan bod. $\mathrm{Na}$ een half jaartje afwezigheid, was ik blij dat je er toch weer terug bent zodat ik je kon lastig vallen met al mijn vragen over promotie/verdediging/paperassen. Maar uiteraard 
vind ik het gewoon ook leuk om die gelijkgestemde ziel weer in de buurt te hebben! Zullen we eens een keertje gaan dansen? :- Gonda, ik heb iedere keer genoten van onze reisjes, terrasjes, filmpjes... Hopelijk hebben we binnenkort weer terug wat meer tijd om wat vaker af te spreken (met 2 of met 4 aan tafel ;))! Ook jij heel veel succes met je promotietraject. Dank jullie wel dat jullie mijn paranimfen wilden zijn!

De andere (oud)-AIO's/postdocs: Nicole, jij weet als geen ander dat je met mij geen grapjes moet uithalen want voordat je het weet ben je carnavalsprinses bij Klinische Genetica (:; Bianca, jij zorgde ervoor dat ik snel ingewerkt was in het mito/POLGwereldje; Lars, Mr. MSN-afkortingen, net gemist op de kamer maar dat betekent niet dat we geen leuke carnaval en pinkpop momenten hebben beleefd; Rudy, heeft mijn Vlaamse invloed je wat kunnen voorbereiden op je baan in Leuven?; Ruben, Mr. Parafilm-fetisj en 'Harry Potter with the magic wand', I will keep the pussies, you can stick with the balls :; Rita, l'm glad everything returned to normal in the end, good luck finishing your thesis; Mike, Mr. Ochtendhumeur, hopelijk geraak je nog een keertje in de Alpen, de vakantie was (afgezien van die gips) echt super, succes in Finland!; Auke, Mr. Ochtendhumeur 2, als ik nu van voetbal zou houden, was ik altijd up-to-date met jou op de kamer... maar ik hou niet van voetbal!!! :); Abhishek, you were here only shortly but I learned a lot about the Indian way of life, for example... Indian people can appreciate a joke or two (hundred) (:); Minh, you only started your PhD training recently, good luck and enjoy life in the Netherlands! With lots of spring rolls and Vietnamese noodles :-). Allemaal heel erg bedankt! Ze zeggen wel eens, gedeelde smart is halve smart en gedeelde vreugde is dubbele vreugde.

Niet te vergeten zijn natuurlijk alle andere mensen van de afdeling, zowel in UNS50 als in het 3X/Noordgebouw. Rosy, bedankt voor het regelen van allerlei afspraken, papierwerk enzovoort; Patrick, thanks for all the analyses, I know they were not always straight-forward; Fons, zonder jouw hulp met de SeqC data was ik nu waarschijnlijk nog altijd aan het tellen, dankjewel!; Bieke, Erika en Rik, bedankt voor de hulp bij de experimenten en labdingen in het algemeen; Torik, Roselie, Wanwisa, Ellen, Iris, Frank, Barbie, Bart, Jo, Jos, Marion, Sabine, Miroslav, Rob, Ton en Marij, ik zal de afgelopen jaren wel minstens een keer om hulp gevraagd hebben, dank je voor jullie tijd. Robin en Kelly, ik vond het heel leuk om jullie als stagiaires te (mogen) begeleiden. Bedankt voor jullie inzet en veel succes met jullie verdere loopbaan!

Alexandra, ik denk dat ik jouw nummer wel het meest van allemaal heb ingetoetst. Voor mijn gevoel wist jij gewoon bijna altijd alle antwoorden op mijn vragen. Heel erg bedankt! Chantal, Eveline en Debby, jullie horen eveneens tot het mito-clubje en konden ook wel eens door mij gespamd worden. Bedankt voor alle hulp!

Eigenlijk is het al een tijdje geleden dat ik mijn echte AIO-periode heb afgesloten. Daarom wil ik toch nog even alle 'nieuwe' collega's bij Clinical Genomics en Maastro Lab/Clinic willen bedanken voor hun hulp, kennis, collegialiteit en de kansen die ze mij de afgelopen maanden gegeven hebben.

Alle andere co-auteurs die ik nog niet vermeld heb of mensen die ik onbewust vergeten ben: heel erg bedankt, thanks a lot, merci beaucoup! 
De afgelopen jaren draaiden natuurlijk niet enkel om werk. Op de universiteit waren er de koffie pauzes. Antoine, jij bent een aantal jaar mijn koffiemaatje geweest, het zullen inderdaad wel ettelijke liters geweest zijn alles tesamen. Veel succes met je opleiding en niet te vergeten je onmiskenbare observatievermogen, vooral voor vrouwelijk schoon (:) Maar eerlijk gezegd, zonder Papendal had ik je misschien nooit leren kennen. Aanbeland in het Arnhemse zou ik toch ook nog de andere mensen willen bedanken die die 3 weekjes (over)leefbaar gemaakt hebben: kamergenootjes Hilde en Jelly, Kris, Anneleen en Bart, Olivier, en alle anderen... bedankt voor de leuke avonden! Erik, ook jij bedankt voor de leuke BBQ's, PhD parties, borrels...

Ik heb verder het privilege gehad om ondergedompeld te worden in de karatewereld. Sensei Peter (R.I.P.), sensei Ramon en sensei Tamara, ik heb ZO veel van jullie geleerd, karate is zoveel meer dan enkel een gevechtssport, het is een manier van leven. Jullie club voelt aan als een hele hechte groep vrienden, bijna als een familie. Jullie hadden precies in de gaten wanneer ik even een dipje had en konden dan precies de juiste woorden gebruiken om dat weer te overwinnen (ja, motiveren en oppeppen is een van jullie sterke punten). Samen met alle andere karateka, waaronder Charlotte, Irene, Samefko, Vasken... dank jullie wel voor de hele leuke tijd!

Naast de karateclub was er nog een ander clubje dat ik niet mag vergeten. Meisjes die elkaar kennen van de universiteit. Maar kleine meisjes worden groot! Ann, Kim, Katrien en Greet, ik vond het echt leuk dat we zijn blijven afspreken, dank jullie voor de gezellige momenten. Ook al is het de laatste tijd wat minder geweest door omstandigheden, we moeten dit zeker in ere houden!

Ine, een zoveelste prettig gestoorde Belg in Maastricht. Ach ja, gek zijn doet geen pijn he $:-$ Het klikte meteen tussen ons en woorden waren vaak niet nodig om duidelijk te maken wat we bedoelden. Ook al woon je nu in Washington, ik hoop dat we regelmatig contact zullen blijven houden. Dankjewel voor de leuke tijd!

Lisanne en Dennis: het is echt wel gezellig dat we van het nieuwjaarsetentje een (twee) maandelijks evenement hebben kunnen maken. Dank jullie voor de gekke toestanden en niet-voor-publicatie foto's!

Er zijn twee mensen zonder wie dit hele verhaal niet mogelijk was geweest: mama en papa. Bedankt dat jullie me de kans gegeven hebben om te gaan studeren en erna nog even te blijven plakken totdat ik op mijn eigen benen kon staan. Ook al begrijpen jullie waarschijnlijk niet veel van wat er in dit boekje allemaal staat, ik ben jullie heel erg dankbaar voor alle wijsheid die jullie mij tot nog toe hebben bijgebracht en alle steun die ik aan jullie gehad heb. Hopelijk zijn jullie even trots op mij als ik ben op jullie! Ben, grote kleine broer, ook al kwamen we niet altijd even goed overeen, ik weet dat ik toch altijd bij je terecht kon/kan als dat nodig was/is.

Als laatste maar zeker niet de minste, Ben(jamin), je hebt me leren kennen in een (voor mij) nogal stressvolle periode waarin ik waarschijnlijk niet altijd even veel tijd voor je had. Dankjewel voor je geduld en je steun! Nu meer tijd voor ons.

\section{An}



Curriculum vitae

\section{List of publications}




\section{Curriculum Vitae}

An Mieke Voets werd geboren op 8 juni 1984 te Bilzen. Van 1996 tot 2002 doorliep zij het Algemeen Secundair Onderwijs (ASO) aan de Onze Lieve Vrouwe Humaniora te Tongeren. $\mathrm{Na}$ het behalen van haar middelbare school diploma in de richting Wetenschappen-Wiskunde in 2002, startte ze datzelfde jaar met de Bacheloropleiding Biomedische Wetenschappen aan de Universiteit Hasselt/transnationale Universiteit Limburg. In 2005 liep ze korte stages bij het Biomedisch Onderzoeksinstituut van de Universiteit Hasselt en de afdeling Gezondheidsrisicoanalyse en Toxicologie van de Universiteit Maastricht en behaalde ze haar Bachelor diploma met grote onderscheiding. Gedurende het volgende jaar volgde ze de Masteropleiding Biomedische Wetenschappen aan de Universiteit Hasselt/transnationale Universiteit Limburg. Haar afstudeerstage bij het Biomedisch Onderzoeksinstituut situeerde zich rond het identificeren van nieuwe diagnostische merkers voor Multiple Sclerose en leidde tot het behalen van het Master diploma Biomedische Wetenschappen met onderscheiding in 2006. Geprikkeld door moleculaire levenswettenschappen, met name genetica, en met de wil onderzoekservaring op te doen, startte ze haar promotietraject aan de Universiteit Maastricht bij de afdeling Clinical Genomics (voormalig Populatiegenetica), verbonden aan de Cardiovascular Research School CARIM. Het promotieonderzoek was gericht op genetische mitochondriële aandoeningen met het doel modellen te creëren ter bestudering van de pathofysiologie van deze aandoeningen en/of ter evaluatie van kandidaat therapeutische stoffen. Tijdens deze periode heeft ze tevens meerdere cursussen gevolgd ter behaling van o.a. het CARIM certificaat voor promovendi en deskundigheidsniveau $5 \mathrm{~b}$ voor stralingshygiëne. Sinds het voorjaar 2011 is zij betrokken bij een gezamelijke studie van de afdeling Klinische Genetica en Maastro Lab/Clinic die de link tussen mitochondria/mitochondrieel DNA en stralingsgevoeligheid moet verduidelijken. Vanaf september 2011 combineerde zij dit onderzoek met DNA-diagnostiek van erfelijke (borst)kanker. 


\section{Publications}

Bonneux S, Fransen E, Van Eyken E, Van Laer L, Huyghe J, Van de Heyning P, Voets A, Gerards M, Stassen AP, Hendrickx AT, Smeets HJ, Van Camp G. Inherited mitochondrial variants are not a major cause of age-related hearing impairment in the European population. Mitochondrion 2011; 11:729-734.

Voets AM, van den Bosch BJC, Stassen AP, Hendrickx AT, Hellebrekers DM, Van Laer L, Van Eycken E, Van Camp G, Pyle A, Baudouin SV, Chinnery PF, Smeets HJM. Large scale mtDNA sequencing reveals sequence and functional conservation as major determinants of homoplasmic mtDNA variant distribution. Mitochondrion 2011; 11: 964972.

Voets AM, Huigsloot M, Lindsey PJ, Leenders AM, Rodenburg RJ, Koopman WJ, Willems PH, Smeitink JA, Smeets HJ. Transcriptional changes in OXPHOS complex I deficiency are related to antioxidant pathways and could explain the disturbed calcium homeostasis. In press, BBA - Molecular basis of disease.

Voets AM, van den Bosch BJC, Lindsey PJ, Verdijk R, Verheyen F, Vanherle SJ, Schoonderwoerd GC, de Die-Smulders CE, Poll-The BT, de Visser M, Faber CG, de Coo IFM, Smeets HJM. POLG1 defects lead to mitochondrial muscle abnormalities, oxidative stress in fibroblasts and apoptosis in brain and liver. Submitted.

Voets AM, Esseling JJ, Lindsey PJ, Schoonderwoerd GC, Godschalk RW, Koopman W, Willems $P$, de Coo IFM, Smeets HJM. Patient-derived fibroblasts indicate oxidative stress status and may justify antioxidant therapy in OXPHOS disorders. Submitted.

Voets AM, Dehing-Oberije C, Struijk RB, De Ruyck K, Thierens H, Vandecasteele K, De Neve W, De Ruysscher D, Smeets HJM, Lambin P. No association between TGF$\beta 1$ polymorphisms and radiation-induced lung toxicity in a European cohort of lung cancer patients. Submitted. 


\section{Abstracts}

Voets AM, van den Bosch BJC, Blok MJ, de Coo IFM, Smeets HJM. Mutations in the polymerase gamma 1 gene negatively influence energy production and lead to compensatory mechanisms in human and mouse.

Poster presentation VLAG Nutrigenomics Master Class 2007, Wageningen, NL Poster presentation NHS PhD training course 2007, Papendal, NL Oral presentation Genetica Retraite 2008, Rolduc Kerkrade, NL

Voets AM, van den Bosch BJC, de Coo IFM, Smeets HJM. Pathophysiological processes involved in mitochondrial disorders and ageing. Poster presentation NHS PhD training course 2008, Papendal, NL

Voets AM, Cleutjens JP, Lindsey PL, de Coo IFM, Smeets HJM. Oxidative stress in OXPHOS disorders depends on the genetic defect.

Poster presentation NHS PhD training course 2009, Papendal, NL Poster presentation FEBS advanced lecture course 2009, Antalya, TR

Voets AM, Otten ABC, van den Bosch BJC, van Eijsden RGE, Lindsey P, Winandy M, de Coo IFM, Smeets HJM. Human and zebrafish models to study cardiac mitochondrial disease from cause to treatment.

Poster presentation CARIM external cluster review, Maastricht, NL Poster presentation CARIM symposium 2010, Maastricht, NL 


\section{Abbreviations}




$\begin{array}{ll}\text { ADP } & \text { adenosine di-phosphate } \\ \text { AIC } & \text { Akaike information criterion } \\ \text { ATP } & \text { adenosine tri-phosphate } \\ \text { CAT } & \text { catalase } \\ \text { Cl } & \text { complex I } \\ \text { COX } & \text { cytochrome C oxidase } \\ \text { D-loop } & \text { displacement loop } \\ \text { DNA } & \text { deoxyribonucleic acid } \\ \text { EM } & \text { electron microscopy } \\ \text { ETC } & \text { electron transport chain } \\ \text { FAD } / F A D H 2 & \text { flavin adenine dinucleotide } \\ \text { GSEQ } & \text { Genechip Sequence Analysis Software (Affymetrix) } \\ \mathrm{H}_{2} \mathrm{O}_{2} & \text { hydrogen peroxide } \\ \text { IHC } & \text { immunohistochemistry } \\ \text { KSS } & \text { Kearns Sayre syndrome } \\ \text { LHON } & \text { Leber hereditery optic neuropathy } \\ \text { LS } & \text { Leigh syndrome } \\ \text { MDS } & \text { mitochondrial depletion syndrome } \\ \text { MELAS } & \text { mitochondrial encephalomyopathy, lactic acidosis and } \quad \text { stroke-like } \\ & \text { episodes } \\ \text { MERRF } & \text { myoclonus epilepsy with ragged red fibers } \\ \text { MNGIE } & \text { mitochondrial neurogastrointestinal encephalomyopathy } \\ \text { mRNA } & \text { messenger ribonucleic acid } \\ \text { mtDNA } & \text { mitochondrial DNA } \\ \text { NAD } / \text { NADH } & \text { nicotinamide adenine dinucleotide } \\ \text { NARP } & \text { neuropathy, ataxia and retinitis pigmentosa } \\ \text { nDNA } & \text { nuclear DNA } \\ \mathrm{O}_{2}^{--} & \text {superoxide } \\ \text { OH } & \text { hydroxyl radical } \\ \text { OXPHOS } & \text { oxidative phosphorylation } \\ \text { PEO } & \text { progressive external ophtalmoplegia } \\ \text { Pol Y } & \text { polymerase gamma } \\ \text { QPCR } & \text { quantitative polymerase chain reaction } \\ \text { rCRS } & \text { revised Cambridge Reference Sequence } \\ \text { ROS } & \text { reactive oxygen species } \\ \text { RRF } & \text { ragged red fibers } \\ \text { rRNA } & \text { ribosomal ribonucleic acid } \\ \text { SDH } & \text { succinate dehydrogenase } \\ \text { SeqC } & \text { Sequence Pilot }- \text { module C } \text { (JSI) } \\ \text { SOD } & \text { superoxide dismutase } \\ \text { tRNA } & \text { transfer ribosomal ribonucleic acid } \\ \text { VPA } & \text { valproic acid } \\ \text { WC } & \text { Watson-Crick } \\ & \end{array}$


Appendix 
Supplementary table 3.1. Significantly differentially expressed genes in muscle of POLG1 patients.

\begin{tabular}{|c|c|c|c|}
\hline Ensemble ID & fc & GeneSymbol & Description \\
\hline ENSG00000130600 & 0.37 & $\mathrm{H} 19$ & $\begin{array}{l}\mathrm{H} 19 \text {, imprinted maternally expressed transcript (non-protein } \\
\text { coding) }\end{array}$ \\
\hline ENSG00000165312 & 0.42 & OTUD1 & OTU domain containing 1 \\
\hline ENSG00000175567 & 0.45 & UCP2 & Uncoupling protein 2 (mitochondrial, proton carrier) \\
\hline ENSG00000124588 & 0.49 & NQO2 & $\mathrm{NAD}(\mathrm{P}) \mathrm{H}$ dehydrogenase, quinone 2 \\
\hline ENSG00000129250 & 0.49 & KIF1C & Kinesin family member $1 \mathrm{C}$ \\
\hline ENSG00000169047 & 0.53 & IRS1 & Insulin receptor substrate 1 \\
\hline ENSG00000198925 & 0.56 & ATG9A & ATG9 autophagy related 9 homolog A (S. cerevisiae) \\
\hline ENSG00000204574 & 0.56 & ABCF1 & ATP-binding cassette, sub-family F (GCN20), member 1 \\
\hline ENSG00000177731 & 0.56 & FLII & Flightless I homolog (Drosophila) \\
\hline ENSG00000133454 & 0.56 & MYO18B & Myosin XVIIIB \\
\hline ENSG00000120709 & 0.56 & FAM53C & Family with sequence similarity 53 , member $\mathrm{C}$ \\
\hline ENSG00000197905 & 0.56 & TEAD4 & TEA domain family member 4 \\
\hline ENSG00000114999 & 0.57 & TTL & Tubulin tyrosine ligase \\
\hline ENSG00000108823 & 0.57 & SGCA & Sarcoglycan, alpha (50kDa dystrophin-associated glycoprotein) \\
\hline ENSG00000103264 & 0.57 & FBXO31 & F-box protein 31 \\
\hline ENSG00000144868 & 0.58 & TMEM108 & Transmembrane protein 108 \\
\hline ENSG00000164068 & 0.58 & RNF123 & Ring finger protein 123 \\
\hline ENSG00000079999 & 0.58 & KEAP1 & Kelch-like ECH-associated protein 1 \\
\hline ENSG00000204463 & 0.59 & BAT3 & HLA-B associated transcript 3 \\
\hline ENSG00000089693 & 0.59 & MLF2 & Myeloid leukemia factor 2 \\
\hline ENSG00000143321 & 0.60 & HDGF & $\begin{array}{l}\text { Hepatoma-derived growth factor (high-mobility group protein } 1 \text { - } \\
\text { like) }\end{array}$ \\
\hline ENSG00000148229 & 0.60 & POLE3 & Polymerase (DNA directed), epsilon 3 ( $\mathrm{p} 17$ subunit) \\
\hline ENSG00000106070 & 0.60 & GRB10 & Growth factor receptor-bound protein 10 \\
\hline ENSG00000034152 & 0.60 & MAP2K3 & Mitogen-activated protein kinase kinase 3 \\
\hline ENSG00000123700 & 0.61 & KCNJ2 & Potassium inwardly-rectifying channel, subfamily J, member 2 \\
\hline ENSG00000156804 & 0.61 & FBXO32 & F-box protein 32 \\
\hline ENSG00000102935 & 0.61 & ZNF423 & Zinc finger protein 423 \\
\hline ENSG00000129245 & 0.62 & FXR2 & Fragile X mental retardation, autosomal homolog 2 \\
\hline ENSG00000134644 & 0.62 & PUM1 & Pumilio homolog 1 (Drosophila) \\
\hline ENSG00000141965 & 0.62 & FEM1A & Fem-1 homolog a (C. elegans) \\
\hline ENSG00000182446 & 0.62 & NPLOC4 & Nuclear protein localization 4 homolog (S. cerevisiae) \\
\hline ENSG00000111046 & 0.63 & MYF6 & Myogenic factor 6 (herculin) \\
\hline ENSG00000165916 & 0.63 & PSMC3 & Proteasome (prosome, macropain) 26S subunit, ATPase, 3 \\
\hline ENSG00000197746 & 0.63 & PSAP & Prosaposin \\
\hline ENSG00000087191 & 0.63 & PSMC5 & Proteasome (prosome, macropain) 26S subunit, ATPase, 5 \\
\hline ENSG00000186350 & 0.63 & $\mathrm{R} \times \mathrm{RA}$ & Retinoid $X$ receptor, alpha \\
\hline ENSG00000103994 & 0.63 & ZFP106 & Zinc finger protein 106 homolog (mouse) \\
\hline ENSG00000189091 & 0.63 & SF3B3 & Splicing factor $3 \mathrm{~b}$, subunit $3,130 \mathrm{kDa}$ \\
\hline ENSG00000136247 & 0.63 & ZDHHC4 & Zinc finger, DHHC-type containing 4 \\
\hline
\end{tabular}




\begin{tabular}{|c|c|c|c|}
\hline Ensemble ID & fc & GeneSymbol & Description \\
\hline ENSG00000111276 & 0.63 & CDKN1B & Cyclin-dependent kinase inhibitor 1B (p27, Kip1) \\
\hline ENSG00000112245 & 0.64 & PTP4A1 & Protein tyrosine phosphatase type IVA, member 1 \\
\hline ENSG00000102572 & 0.64 & STK24 & Serine/threonine kinase 24 (STE20 homolog, yeast) \\
\hline ENSG00000092607 & 0.64 & TBX15 & T-box 15 \\
\hline ENSG00000122971 & 0.64 & ACADS & Acyl-Coenzyme A dehydrogenase, C-2 to C-3 short chain \\
\hline ENSG00000130749 & 0.64 & CS007_HUMAN & Zinc finger $\mathrm{CCCH}$ domain-containing protein $\mathrm{C} 19$ orf7 \\
\hline ENSG00000182108 & 0.64 & MYLE_HUMAN & Protein MYLE (Dexamethasone-induced protein) \\
\hline ENSG00000165795 & 0.65 & NDRG2 & NDRG family member 2 \\
\hline ENSG00000107829 & 0.65 & FBXW4 & F-box and WD repeat domain containing 4 \\
\hline ENSG00000036448 & 0.65 & MYOM2 & Myomesin (M-protein) 2, 165kDa \\
\hline ENSG00000119242 & 0.65 & CCDC92 & Coiled-coil domain containing 92 \\
\hline ENSG00000145901 & 0.65 & TNIP1 & TNFAIP3 interacting protein 1 \\
\hline ENSG00000198954 & 0.66 & KIAA1279 & KIAA1279 \\
\hline ENSG00000179364 & 0.66 & PACS2 & Phosphofurin acidic cluster sorting protein 2 \\
\hline ENSG00000144579 & 0.66 & CTDSP1 & $\begin{array}{l}\text { CTD (carboxy-terminal domain, RNA polymerase II, polypeptide } \\
\text { A) small phosphatase } 1\end{array}$ \\
\hline ENSG00000125827 & 0.66 & TXNDC13 & Thioredoxin domain-containing protein 13 precursor \\
\hline ENSG00000143761 & 0.66 & ARF1 & ADP-ribosylation factor 1 \\
\hline ENSG00000134590 & 0.66 & FAM127A & Family with sequence similarity 127 , member $\mathrm{A}$ \\
\hline ENSG00000107372 & 0.66 & ZFAND5 & Zinc finger, AN1-type domain 5 \\
\hline ENSG00000171497 & 0.66 & PPID & Peptidylprolyl isomerase D \\
\hline ENSG00000159692 & 0.67 & CTBP1 & C-terminal binding protein 1 \\
\hline ENSG00000138668 & 0.67 & HNRPD & Heterogeneous nuclear ribonucleoprotein D0 \\
\hline ENSG00000169564 & 0.67 & PCBP1 & Poly $(\mathrm{rC})$ binding protein 1 \\
\hline ENSG00000095139 & 0.67 & ARCN1 & Archain 1 \\
\hline ENSG00000084463 & 0.67 & WBP11 & WW domain binding protein 11 \\
\hline ENSG00000107959 & 0.67 & PITRM1 & Pitrilysin metallopeptidase 1 \\
\hline ENSG00000115677 & 0.67 & HDLBP & High density lipoprotein binding protein \\
\hline ENSG00000099942 & 0.67 & CRKL & Crk-like protein \\
\hline ENSG00000168575 & 0.67 & SLC20A2 & Solute carrier family 20 (phosphate transporter), member 2 \\
\hline ENSG00000173545 & 0.67 & ZNF622 & Zinc finger protein 622 \\
\hline ENSG00000100714 & 0.67 & MTHFD1 & $\begin{array}{l}\text { Methylenetetrahydrofolate dehydrogenase (NADP+ dependent) } \\
1 \text {, methenyltetrahydrofolate cyclohydrolase, } \\
\text { formyltetrahydrofolate synthetase }\end{array}$ \\
\hline ENSG00000101400 & 0.67 & SNTA1 & $\begin{array}{l}\text { Syntrophin, alpha } 1 \text { (dystrophin-associated protein } \mathrm{A} 1,59 \mathrm{kDa} \text {, } \\
\text { acidic component) }\end{array}$ \\
\hline ENSG00000123815 & 0.67 & ADCK4 & AarF domain containing kinase 4 \\
\hline ENSG00000159720 & 0.67 & ATP6V0D1 & ATPase, $\mathrm{H}+$ transporting, lysosomal $38 \mathrm{kDa}, \mathrm{V} 0$ subunit d1 \\
\hline ENSG00000122884 & 0.67 & P4HA1 & Prolyl 4-hydroxylase, alpha polypeptide I \\
\hline ENSG00000130957 & 0.67 & FBP2 & Fructose-1,6-bisphosphatase 2 \\
\hline ENSG00000182533 & 0.67 & CAV3 & Caveolin 3 \\
\hline ENSG00000185052 & 0.68 & SLC24A3 & $\begin{array}{l}\text { Solute carrier family } 24 \text { (sodium/potassium/calcium exchanger), } \\
\text { member } 3\end{array}$ \\
\hline ENSG00000078804 & 0.68 & TP53INP2 & Tumor protein p53 inducible nuclear protein 2 \\
\hline ENSG00000160094 & 0.68 & ZNF362 & Zinc finger protein 362 \\
\hline ENSG00000065675 & 0.68 & PRKCQ & Protein kinase $\mathrm{C}$, theta \\
\hline
\end{tabular}




\begin{tabular}{|c|c|c|c|}
\hline Ensemble ID & fc & GeneSymbol & Description \\
\hline ENSG00000096384 & 0.68 & HSP90AB1 & Heat shock protein 90kDa alpha (cytosolic), class B member 1 \\
\hline ENSG00000104341 & 0.68 & LAPTM4B & Lysosomal protein transmembrane 4 beta \\
\hline ENSG00000197043 & 0.68 & ANXA6 & Annexin A6 \\
\hline ENSG00000204310 & 0.68 & AGPAT1 & $\begin{array}{l}\text { 1-acylglycerol-3-phosphate O-acyltransferase } 1 \\
\text { (lysophosphatidic acid acyltransferase, alpha) }\end{array}$ \\
\hline ENSG00000004142 & 0.68 & POLDIP2 & Polymerase (DNA-directed), delta interacting protein 2 \\
\hline ENSG00000015676 & 0.68 & NUDCD3 & NudC domain containing 3 \\
\hline ENSG00000082641 & 0.68 & NFE2L1 & Nuclear factor (erythroid-derived 2)-like 1 \\
\hline ENSG00000103319 & 0.68 & EEF2K & Eukaryotic elongation factor-2 kinase \\
\hline ENSG00000065060 & 0.68 & C6orf107 & UHRF1-binding protein 1 \\
\hline ENSG00000107140 & 0.68 & TESK1 & Testis-specific kinase 1 \\
\hline ENSG00000188636 & 0.69 & LDOC1L & Leucine zipper, down-regulated in cancer 1-like \\
\hline ENSG00000102974 & 0.69 & CTCF & CCCTC-binding factor (zinc finger protein) \\
\hline ENSG00000164062 & 0.69 & APEH & $\mathrm{N}$-acylaminoacyl-peptide hydrolase \\
\hline ENSG00000197321 & 0.69 & SVIL & Supervillin \\
\hline ENSG00000130764 & 0.69 & LRRC47 & Leucine rich repeat containing 47 \\
\hline ENSG00000167182 & 0.69 & SP2 & Sp2 transcription factor \\
\hline ENSG00000174243 & 0.69 & DDX23 & DEAD (Asp-Glu-Ala-Asp) box polypeptide 23 \\
\hline ENSG00000177728 & 0.69 & KIAA0195 & KIAA0195 \\
\hline ENSG00000007314 & 0.69 & SCN4A & Sodium channel, voltage-gated, type IV, alpha subunit \\
\hline ENSG00000185909 & 0.69 & KLHDC8B & Kelch domain containing $8 \mathrm{~B}$ \\
\hline ENSG00000159346 & 0.69 & ADIPOR1 & Adiponectin receptor 1 \\
\hline ENSG00000109775 & 0.69 & C4orf20 & CDNA FLJ11200 fis, clone PLACE1007725 \\
\hline ENSG00000125304 & 0.69 & TM9SF2 & Transmembrane 9 superfamily member 2 \\
\hline ENSG00000108639 & 0.69 & SYNGR2 & Synaptogyrin 2 \\
\hline ENSG00000078618 & 0.69 & NRD1 & Nardilysin (N-arginine dibasic convertase) \\
\hline ENSG00000111481 & 0.69 & COPZ1 & Coatomer protein complex, subunit zeta 1 \\
\hline ENSG00000113141 & 0.69 & IK & IK cytokine, down-regulator of HLA II \\
\hline ENSG00000114853 & 0.69 & ZBTB47 & Zinc finger and BTB domain containing 47 \\
\hline ENSG00000103502 & 0.69 & CDIPT & $\begin{array}{l}\text { CDP-diacylglycerol--inositol 3-phosphatidyltransferase } \\
\text { (phosphatidylinositol synthase) }\end{array}$ \\
\hline ENSG00000158828 & 0.69 & PINK1 & PTEN induced putative kinase 1 \\
\hline ENSG00000105968 & 0.69 & H2AFV & $\mathrm{H} 2 \mathrm{~A}$ histone family, member $\mathrm{V}$ \\
\hline ENSG00000073969 & 0.69 & NSF & $\mathrm{N}$-ethylmaleimide-sensitive factor \\
\hline ENSG00000134686 & 0.69 & PHC2 & Polyhomeotic homolog 2 (Drosophila) \\
\hline ENSG00000136891 & 0.69 & TEX10 & Testis expressed 10 \\
\hline ENSG00000090097 & 0.70 & PCBP4 & Poly $(\mathrm{rC})$ binding protein 4 \\
\hline ENSG00000132471 & 0.70 & WBP2 & WW domain binding protein 2 \\
\hline ENSG00000185000 & 0.70 & DGAT1 & Diacylglycerol O-acyltransferase 1 \\
\hline ENSG00000132383 & 0.70 & RPA1 & Replication protein $\mathrm{A} 1,70 \mathrm{kDa}$ \\
\hline ENSG00000136451 & 0.70 & VEZF1 & Vascular endothelial zinc finger 1 \\
\hline ENSG00000133606 & 0.70 & MKRN1 & Makorin ring finger protein 1 \\
\hline ENSG00000004487 & 0.70 & AOF2 & Flavin-containing amine oxidase domain-containing protein 2 \\
\hline ENSG00000125534 & 0.70 & C20orf149 & Chromosome 20 open reading frame 149 \\
\hline
\end{tabular}




\begin{tabular}{|c|c|c|c|}
\hline Ensemble ID & fc & GeneSymbol & Description \\
\hline ENSG00000135624 & 0.70 & CCT7 & Chaperonin containing TCP1, subunit 7 (eta) \\
\hline ENSG00000161202 & 0.70 & DVL3 & Dishevelled, dsh homolog 3 (Drosophila) \\
\hline ENSG00000036257 & 0.70 & CUL3 & Cullin 3 \\
\hline ENSG00000103150 & 0.70 & MLYCD & Malonyl-CoA decarboxylase \\
\hline ENSG00000120438 & 0.70 & TCP1 & T-complex 1 \\
\hline ENSG00000176087 & 0.70 & SLC35A4 & Solute carrier family 35 , member A4 \\
\hline ENSG00000156515 & 0.70 & HK1 & Hexokinase 1 \\
\hline ENSG00000180304 & 0.70 & OAZ2 & Ornithine decarboxylase antizyme 2 \\
\hline ENSG00000144028 & 0.70 & ASCC3L1 & Activating signal cointegrator 1 complex subunit 3-like 1 \\
\hline ENSG00000178764 & 0.70 & ZHX2 & Zinc fingers and homeoboxes protein 2 \\
\hline ENSG00000119335 & 0.70 & SET & Protein SET (Phosphatase 2A inhibitor I2PP2A) (I-2PP2A) \\
\hline ENSG00000141699 & 0.70 & NP_835227.1 & NA \\
\hline ENSG00000108270 & 0.70 & AATF & Apoptosis antagonizing transcription factor \\
\hline ENSG00000047249 & 0.70 & ATP6V1H & ATPase, $\mathrm{H}+$ transporting, lysosomal $50 / 57 \mathrm{kDa}, \mathrm{V} 1$ subunit $\mathrm{H}$ \\
\hline ENSG00000138363 & 0.70 & ATIC & $\begin{array}{l}\text { 5-aminoimidazole-4-carboxamide ribonucleotide } \\
\text { formyltransferase/IMP cyclohydrolase }\end{array}$ \\
\hline ENSG00000163431 & 0.70 & LMOD1 & Leiomodin 1 (smooth muscle) \\
\hline ENSG00000204619 & 0.70 & PPP1R11 & Protein phosphatase 1, regulatory (inhibitor) subunit 11 \\
\hline ENSG00000114867 & 0.70 & EIF4G1 & Eukaryotic translation initiation factor 4 gamma, 1 \\
\hline ENSG00000126457 & 0.71 & PRMT1 & Protein arginine methyltransferase 1 \\
\hline ENSG00000006125 & 0.71 & AP2B1 & Adaptor-related protein complex 2 , beta 1 subunit \\
\hline ENSG00000104848 & 0.71 & KCNA7 & $\begin{array}{l}\text { Potassium voltage-gated channel, shaker-related subfamily, } \\
\text { member } 7\end{array}$ \\
\hline ENSG00000153827 & 0.71 & TRIP12 & Thyroid hormone receptor interactor 12 \\
\hline ENSG00000136813 & 0.71 & KIAA0368 & Proteasome-associated protein ECM29 homolog \\
\hline ENSG00000162104 & 0.71 & ADCY9 & Adenylate cyclase 9 \\
\hline ENSG00000124299 & 0.71 & PEPD & Peptidase D \\
\hline ENSG00000177105 & 0.71 & $\mathrm{RHOG}$ & Ras homolog gene family, member G (rho G) \\
\hline ENSG00000111897 & 0.71 & SERINC1 & Serine incorporator 1 \\
\hline ENSG00000177189 & 0.71 & RPS6KA3 & Ribosomal protein S6 kinase, $90 \mathrm{kDa}$, polypeptide 3 \\
\hline ENSG00000185551 & 0.71 & $\mathrm{NR} 2 \mathrm{~F} 2$ & Nuclear receptor subfamily 2 , group F, member 2 \\
\hline ENSG00000108515 & 0.71 & ENO3 & Enolase 3 (beta, muscle) \\
\hline ENSG00000012822 & 0.71 & CALCOCO1 & Calcium binding and coiled-coil domain 1 \\
\hline ENSG00000078061 & 0.71 & ARAF & A-Raf proto-oncogene serine/threonine-protein kinase \\
\hline ENSG00000113048 & 0.71 & MRPS27 & Mitochondrial ribosomal protein S27 \\
\hline ENSG00000198676 & 0.71 & Q7Z4H1_HUMAN & HBeAg-binding protein 1 \\
\hline ENSG00000197451 & 0.72 & HNRPAB & Heterogeneous nuclear ribonucleoprotein $A / B$ \\
\hline ENSG00000065970 & 0.72 & FOXJ2 & Forkhead box J2 \\
\hline ENSG00000077549 & 0.72 & CAPZB & Capping protein (actin filament) muscle Z-line, beta \\
\hline ENSG00000115806 & 0.72 & GORASP2 & Golgi reassembly stacking protein $2,55 \mathrm{kDa}$ \\
\hline ENSG00000122203 & 0.72 & KIAA1191 & KIAA1191 \\
\hline ENSG00000188130 & 0.72 & MAPK12 & Mitogen-activated protein kinase 12 \\
\hline ENSG00000188677 & 0.72 & PARVB & Parvin, beta \\
\hline ENSG00000198837 & 0.72 & DENND4B & DENN/MADD domain containing 4B \\
\hline
\end{tabular}




\begin{tabular}{|c|c|c|c|}
\hline Ensemble ID & fc & GeneSymbol & Description \\
\hline ENSG00000100632 & 0.72 & $\mathrm{ERH}$ & Enhancer of rudimentary homolog (Drosophila) \\
\hline ENSG00000119950 & 0.72 & MXI1 & MAX interactor 1 \\
\hline ENSG00000114544 & 0.72 & SLC41A3 & Solute carrier family 41 , member 3 \\
\hline ENSG00000185883 & 0.72 & ATP6V0C & ATPase, $\mathrm{H}+$ transporting, lysosomal $16 \mathrm{kDa}, \mathrm{V} 0$ subunit c \\
\hline ENSG00000104812 & 0.72 & GYS1 & Glycogen synthase 1 (muscle) \\
\hline ENSG00000100109 & 0.72 & TFIP11 & Tuftelin interacting protein 11 \\
\hline ENSG00000100412 & 0.72 & $\mathrm{ACO} 2$ & Aconitase 2 , mitochondrial \\
\hline ENSG00000115657 & 0.72 & ABCB6 & ATP-binding cassette, sub-family B (MDR/TAP), member 6 \\
\hline ENSG00000131462 & 0.72 & TUBG1 & Tubulin, gamma 1 \\
\hline ENSG00000115840 & 0.72 & SLC25A12 & $\begin{array}{l}\text { Solute carrier family } 25 \text { (mitochondrial carrier, Aralar), member } \\
12\end{array}$ \\
\hline ENSG00000137073 & 0.72 & UBAP2 & Ubiquitin associated protein 2 \\
\hline ENSG00000167671 & 0.72 & UBXD1 & UBX domain-containing protein 1 \\
\hline ENSG00000022840 & 0.72 & RNF10 & Ring finger protein 10 \\
\hline ENSG00000185651 & 0.73 & UBE2L3 & Ubiquitin-conjugating enzyme E2L 3 \\
\hline ENSG00000102225 & 0.73 & PCTK1 & PCTAIRE protein kinase 1 \\
\hline ENSG00000143365 & 0.73 & LRRN6D & Nuclear receptor ROR-gamma \\
\hline ENSG00000141646 & 0.73 & SMAD4 & SMAD family member 4 \\
\hline ENSG00000102900 & 0.73 & NUP93 & Nucleoporin 93kDa \\
\hline ENSG00000140718 & 0.73 & NP_001073901.1 & NA \\
\hline ENSG00000142186 & 0.73 & SCYL1 & SCY1-like 1 (S. cerevisiae) \\
\hline ENSG00000179262 & 0.73 & RAD23A & RAD23 homolog A (S. cerevisiae) \\
\hline ENSG00000187446 & 0.73 & CHP1_HUMAN & Calcium-binding protein p22 \\
\hline ENSG00000011007 & 0.73 & TCEB3 & Transcription elongation factor B polypeptide 3 \\
\hline ENSG00000143621 & 0.73 & ILF2 & Interleukin enhancer binding factor $2,45 \mathrm{kDa}$ \\
\hline ENSG00000169221 & 0.73 & TBC1D10B & TBC1 domain family, member 10B \\
\hline ENSG00000139990 & 0.73 & WDR22 & WD repeat domain 22 \\
\hline ENSG00000186204 & 0.73 & CYP4F12 & Cytochrome P450, family 4, subfamily F, polypeptide 12 \\
\hline ENSG00000107404 & 0.73 & DVL1L1 & Segment polarity protein dishevelled homolog DVL-1 \\
\hline ENSG00000111652 & 0.73 & COPS7A & $\begin{array}{l}\text { COP9 constitutive photomorphogenic homolog subunit 7A } \\
\text { (Arabidopsis) }\end{array}$ \\
\hline ENSG00000112305 & 0.73 & SMAP1 & Small ArfGAP 1 \\
\hline ENSG00000182500 & 0.73 & TMEM142A & Calcium release-activated calcium channel protein 1 \\
\hline ENSG00000144659 & 0.73 & SLC25A38 & Solute carrier family 25, member 38 \\
\hline ENSG00000099821 & 0.73 & POLRMT & Polymerase (RNA) mitochondrial (DNA directed) \\
\hline ENSG00000204389 & 0.73 & HSPA1A & Heat shock $70 \mathrm{kDa}$ protein 1 \\
\hline ENSG00000099622 & 0.73 & CIRBP & Cold inducible RNA binding protein \\
\hline ENSG00000100220 & 0.73 & C22orf28 & Chromosome 22 open reading frame 28 \\
\hline ENSG00000130821 & 0.73 & SLC6A8 & $\begin{array}{l}\text { Solute carrier family } 6 \text { (neurotransmitter transporter, creatine), } \\
\text { member } 8\end{array}$ \\
\hline ENSG00000100347 & 0.73 & SAMM50 & $\begin{array}{l}\text { Sorting and assembly machinery component } 50 \text { homolog (S. } \\
\text { cerevisiae) }\end{array}$ \\
\hline ENSG00000111540 & 0.73 & RAB5B & RAB5B, member RAS oncogene family \\
\hline ENSG00000100242 & 0.73 & UNC84B & Unc-84 homolog B (C. elegans) \\
\hline ENSG00000170153 & 0.73 & RNF150 & Ring finger protein 150 \\
\hline ENSG00000138814 & 0.74 & PPP3CA & $\begin{array}{l}\text { Protein phosphatase } 3 \text { (formerly 2B), catalytic subunit, alpha } \\
\text { isoform }\end{array}$ \\
\hline
\end{tabular}




\begin{tabular}{|c|c|c|c|}
\hline Ensemble ID & fc & GeneSymbol & Description \\
\hline ENSG00000185721 & 0.74 & DRG1 & Developmentally regulated GTP binding protein 1 \\
\hline ENSG00000132716 & 0.74 & WDR42A & WD repeat domain $42 A$ \\
\hline ENSG00000167986 & 0.74 & DDB1 & Damage-specific DNA binding protein $1,127 \mathrm{kDa}$ \\
\hline ENSG00000157020 & 0.74 & SEC13 & SEC13 homolog (S. cerevisiae) \\
\hline ENSG00000102007 & 0.74 & PLP2 & Proteolipid protein 2 (colonic epithelium-enriched) \\
\hline ENSG00000077522 & 0.74 & ACTN2 & Actinin, alpha 2 \\
\hline ENSG00000126247 & 0.74 & CAPNS1 & Calpain, small subunit 1 \\
\hline ENSG00000092203 & 0.74 & KIAA0737 & Epidermal Langerhans cell protein LCP1 \\
\hline ENSG00000145945 & 0.74 & FAM50B & Family with sequence similarity 50 , member $B$ \\
\hline ENSG00000006704 & 0.74 & GTF2IRD1 & GTF2I repeat domain containing 1 \\
\hline ENSG00000196230 & 0.74 & TUBB & Tubulin beta chain \\
\hline ENSG00000159069 & 0.74 & FBXW5 & F-box and WD repeat domain containing 5 \\
\hline ENSG00000197081 & 0.74 & IGF2R & Insulin-like growth factor 2 receptor \\
\hline ENSG00000155189 & 0.74 & AGPAT5 & $\begin{array}{l}\text { 1-acylglycerol-3-phosphate O-acyltransferase } 5 \\
\text { (lysophosphatidic acid acyltransferase, epsilon) }\end{array}$ \\
\hline ENSG00000181929 & 0.74 & PRKAG1 & Protein kinase, AMP-activated, gamma 1 non-catalytic subunit \\
\hline ENSG00000062485 & 0.74 & CS & Citrate synthase \\
\hline ENSG00000179889 & 0.74 & NP_055842.1 & NA \\
\hline ENSG00000164024 & 0.74 & METAP1 & Methionyl aminopeptidase 1 \\
\hline ENSG00000087365 & 0.74 & SF3B2 & Splicing factor $3 \mathrm{~b}$, subunit $2,145 \mathrm{kDa}$ \\
\hline ENSG00000162413 & 0.74 & KLHL21 & Kelch-like 21 (Drosophila) \\
\hline ENSG00000129116 & 0.74 & PALLD & Palladin, cytoskeletal associated protein \\
\hline ENSG00000006757 & 0.74 & PNPLA4 & Patatin-like phospholipase domain containing 4 \\
\hline ENSG00000187555 & 0.74 & USP7 & Ubiquitin specific peptidase 7 (herpes virus-associated) \\
\hline ENSG00000013455 & 0.74 & ARPC1A & Actin related protein $2 / 3$ complex, subunit $1 \mathrm{~A}, 41 \mathrm{kDa}$ \\
\hline ENSG00000123992 & 0.74 & DNPEP & Aspartyl aminopeptidase \\
\hline ENSG00000049245 & 0.74 & VAMP3 & Vesicle-associated membrane protein 3 (cellubrevin) \\
\hline ENSG00000124422 & 0.74 & USP22 & Ubiquitin specific peptidase 22 \\
\hline ENSG00000143870 & 0.74 & PDIA6 & Protein disulfide isomerase family A, member 6 \\
\hline ENSG00000184007 & 0.75 & PTP4A2 & Protein tyrosine phosphatase type IVA, member 2 \\
\hline ENSG00000186187 & 0.75 & ZNRF1 & Zinc and ring finger 1 \\
\hline ENSG00000100462 & 0.75 & PRMT5 & Protein arginine methyltransferase 5 \\
\hline ENSG00000168610 & 0.75 & STAT3 & $\begin{array}{l}\text { Signal transducer and activator of transcription } 3 \text { (acute-phase } \\
\text { response factor) }\end{array}$ \\
\hline ENSG00000166974 & 0.75 & MAPRE2 & Microtubule-associated protein, RP/EB family, member 2 \\
\hline ENSG00000079246 & 0.75 & XRCC5 & $\begin{array}{l}\text { X-ray repair complementing defective repair in Chinese } \\
\text { hamster cells } 5 \text { (double-strand-break rejoining) }\end{array}$ \\
\hline ENSG00000013374 & 0.75 & NUB1 & Negative regulator of ubiquitin-like proteins 1 \\
\hline ENSG00000124214 & 0.75 & STAU1 & Staufen, RNA binding protein, homolog 1 (Drosophila) \\
\hline ENSG00000197170 & 0.75 & PSMD12 & $\begin{array}{l}\text { Proteasome (prosome, macropain) } 26 \mathrm{~S} \text { subunit, non-ATPase, } \\
12\end{array}$ \\
\hline ENSG00000110025 & 0.75 & SNX15 & Sorting nexin 15 \\
\hline ENSG00000166685 & 0.75 & COG1 & Component of oligomeric golgi complex 1 \\
\hline ENSG00000135424 & 0.75 & ITGA7 & Integrin alpha-7 precursor \\
\hline ENSG00000183386 & 0.75 & FHL3 & Four and a half LIM domains 3 \\
\hline ENSG00000095637 & 0.75 & SORBS1 & Sorbin and $\mathrm{SH} 3$ domain containing 1 \\
\hline
\end{tabular}




\begin{tabular}{|c|c|c|c|}
\hline Ensemble ID & fc & GeneSymbol & Description \\
\hline ENSG00000175806 & 0.75 & MSRA & Methionine sulfoxide reductase $A$ \\
\hline ENSG00000169184 & 0.75 & & NA \\
\hline ENSG00000180900 & 0.75 & SCRIB & Scribbled homolog (Drosophila) \\
\hline ENSG00000126368 & 0.75 & NR1D1 & Nuclear receptor subfamily 1 , group $D$, member 1 \\
\hline ENSG00000115525 & 0.75 & ST3GAL5 & ST3 beta-galactoside alpha-2,3-sialyltransferase 5 \\
\hline ENSG00000151327 & 0.75 & C14orf24 & NA \\
\hline ENSG00000146457 & 0.75 & WTAP & Wilms' tumor 1 -associating protein \\
\hline ENSG00000175591 & 0.75 & P2RY2 & Purinergic receptor P2Y, G-protein coupled, 2 \\
\hline ENSG00000166337 & 0.75 & TAF10 & $\begin{array}{l}\text { TAF10 RNA polymerase II, TATA box binding protein (TBP)- } \\
\text { associated factor, } 30 \mathrm{kDa}\end{array}$ \\
\hline ENSG00000145990 & 0.75 & GFOD1 & Glucose-fructose oxidoreductase domain containing 1 \\
\hline ENSG00000176171 & 0.75 & BNIP3 & BCL2/adenovirus E1B 19kDa interacting protein 3 \\
\hline ENSG00000163125 & 0.75 & KIAA0460 & NA \\
\hline ENSG00000163866 & 0.75 & C1orf212 & NA \\
\hline ENSG00000148498 & 0.75 & PARD3 & Par-3 partitioning defective 3 homolog (C. elegans) \\
\hline ENSG00000143702 & 0.75 & CEP170 & Centrosomal protein $170 \mathrm{kDa}$ \\
\hline ENSG00000108468 & 0.75 & CBX1 & Chromobox homolog 1 (HP1 beta homolog Drosophila ) \\
\hline ENSG00000011638 & 0.75 & TMEM159 & Transmembrane protein 159 \\
\hline ENSG00000164054 & 0.75 & NP_057563.3 & scotin \\
\hline ENSG00000185787 & 0.75 & MORF4L1 & Mortality factor 4 like 1 \\
\hline ENSG00000172731 & 0.76 & LRRC20 & Leucine rich repeat containing 20 \\
\hline ENSG00000188554 & 0.76 & NBR1 & neighbor of BRCA1 gene 1 \\
\hline ENSG00000170296 & 0.76 & GABARAP & GABA(A) receptor-associated protein \\
\hline ENSG00000170027 & 0.76 & YWHAG & $\begin{array}{l}\text { Tyrosine 3-monooxygenase/tryptophan 5-monooxygenase } \\
\text { activation protein, gamma polypeptide }\end{array}$ \\
\hline ENSG00000185896 & 0.76 & LAMP1 & Lysosomal-associated membrane protein 1 \\
\hline ENSG00000118639 & 0.76 & RNF103 & Ring finger protein 103 \\
\hline ENSG00000171503 & 0.76 & ETFDH & Electron-transferring-flavoprotein dehydrogenase \\
\hline ENSG00000143457 & 0.76 & GOLPH3L & Golgi phosphoprotein 3-like \\
\hline ENSG00000111716 & 0.76 & LDHB & Lactate dehydrogenase B \\
\hline ENSG00000171867 & 0.76 & PRNP & Major prion protein precursor \\
\hline ENSG00000184678 & 0.76 & HIST2H2BE & Histone cluster 2, H2be \\
\hline ENSG00000060762 & 0.76 & BRP44L & Brain protein 44 -like \\
\hline ENSG00000115216 & 0.76 & NRBP1 & Nuclear receptor binding protein 1 \\
\hline ENSG00000119953 & 0.76 & SMNDC1 & Survival motor neuron domain containing 1 \\
\hline ENSG00000106105 & 0.76 & GARS & Glycyl-tRNA synthetase \\
\hline ENSG00000142046 & 0.76 & TMEM91 & Transmembrane protein 91 \\
\hline ENSG00000144043 & 0.76 & TEX261 & Testis expressed 261 \\
\hline ENSG00000117450 & 0.76 & PRDX1 & Peroxiredoxin 1 \\
\hline ENSG00000167987 & 0.76 & VPS37C & Vacuolar protein sorting 37 homolog $\mathrm{C}$ (S. cerevisiae) \\
\hline ENSG00000163159 & 0.76 & VPS72 & Vacuolar protein sorting 72 homolog (S. cerevisiae) \\
\hline ENSG00000156471 & 0.76 & PTDSS1 & Phosphatidylserine synthase 1 \\
\hline ENSG00000157916 & 0.76 & RER1 & $\begin{array}{l}\text { RER } 1 \text { retention in endoplasmic reticulum } 1 \text { homolog (S. } \\
\text { cerevisiae) }\end{array}$ \\
\hline ENSG00000184916 & 0.76 & JAG2 & Jagged 2 \\
\hline
\end{tabular}




\begin{tabular}{|c|c|c|c|}
\hline Ensemble ID & fc & GeneSymbol & Description \\
\hline ENSG00000113657 & 0.76 & DPYSL3 & Dihydropyrimidinase-like 3 \\
\hline ENSG00000084090 & 0.76 & STARD7 & StAR-related lipid transfer (START) domain containing 7 \\
\hline ENSG00000099875 & 0.76 & MKNK2 & MAP kinase interacting serine/threonine kinase 2 \\
\hline ENSG00000131507 & 0.76 & NDFIP1 & Nedd4 family interacting protein 1 \\
\hline ENSG00000170745 & 0.76 & KCNS3 & $\begin{array}{l}\text { Potassium voltage-gated channel, delayed-rectifier, subfamily } \\
\text { S, member } 3\end{array}$ \\
\hline ENSG00000184489 & 0.76 & PTP4A3 & Protein tyrosine phosphatase type IVA, member 3 \\
\hline ENSG00000135723 & 0.76 & FHOD1 & Formin homology 2 domain containing 1 \\
\hline ENSG00000157881 & 0.76 & PANK4 & Pantothenate kinase 4 \\
\hline ENSG00000168066 & 0.76 & SF1 & Splicing factor 1 \\
\hline ENSG00000174132 & 0.76 & TMEM157 & Transmembrane protein 157 precursor \\
\hline ENSG00000005243 & 0.76 & COPZ2 & Coatomer protein complex, subunit zeta 2 \\
\hline ENSG00000198355 & 0.76 & PIM3 & Pim-3 oncogene \\
\hline ENSG00000162980 & 0.76 & ARL5A & ADP-ribosylation factor-like $5 \mathrm{~A}$ \\
\hline ENSG00000068383 & 0.76 & INPP5A & Inositol polyphosphate-5-phosphatase, $40 \mathrm{kDa}$ \\
\hline ENSG00000157450 & 0.76 & RNF111 & Ring finger protein 111 \\
\hline ENSG00000100522 & 0.76 & GNPNAT1 & Glucosamine-phosphate $\mathrm{N}$-acetyltransferase 1 \\
\hline ENSG00000115484 & 0.76 & CCT4 & Chaperonin containing TCP1, subunit 4 (delta) \\
\hline ENSG00000047315 & 0.76 & POLR2B & Polymerase (RNA) II (DNA directed) polypeptide B, 140kDa \\
\hline ENSG00000130962 & 0.76 & PRRG1 & Proline rich Gla (G-carboxyglutamic acid) 1 \\
\hline ENSG00000148468 & 0.76 & C10orf38 & NA \\
\hline ENSG00000146007 & 0.76 & ZMAT2 & Zinc finger, matrin type 2 \\
\hline ENSG00000197324 & 0.77 & LRP10 & Low density lipoprotein receptor-related protein 10 \\
\hline ENSG00000145495 & 0.77 & Mar-06 & membrane-associated ring finger \\
\hline ENSG00000100897 & 0.77 & WDR23 & WD repeat domain 23 \\
\hline ENSG00000103274 & 0.77 & NUBP1 & Nucleotide binding protein 1 (MinD homolog, E. coli) \\
\hline ENSG00000087111 & 0.77 & PIGS & Phosphatidylinositol glycan anchor biosynthesis, class S \\
\hline ENSG00000145916 & 0.77 & RMND5B & $\begin{array}{l}\text { Required for meiotic nuclear division } 5 \text { homolog B (S. } \\
\text { cerevisiae) }\end{array}$ \\
\hline ENSG00000162144 & 0.77 & CYBASC3 & Cytochrome b, ascorbate dependent 3 \\
\hline ENSG00000103342 & 0.77 & GSPT1 & G1 to S phase transition 1 \\
\hline ENSG00000109971 & 0.77 & HSPA8 & Heat shock $70 \mathrm{kDa}$ protein 8 \\
\hline ENSG00000116350 & 0.77 & SFRS4 & Splicing factor, arginine/serine-rich 4 \\
\hline ENSG00000142684 & 0.77 & ZNF593 & Zinc finger protein 593 \\
\hline ENSG00000119318 & 0.77 & RAD23B & RAD23 homolog B (S. cerevisiae) \\
\hline ENSG00000145391 & 0.77 & SETD7 & SET domain containing (lysine methyltransferase) 7 \\
\hline ENSG00000106554 & 0.77 & $\mathrm{CHCHD} 3$ & Coiled-coil-helix-coiled-coil-helix domain containing 3 \\
\hline ENSG00000137996 & 0.77 & RTCD1 & RNA terminal phosphate cyclase domain 1 \\
\hline ENSG00000109332 & 0.77 & UBE2D3 & Ubiquitin-conjugating enzyme E2D 3 (UBC4/5 homolog, yeast) \\
\hline ENSG00000175216 & 0.77 & CKAP5 & Cytoskeleton associated protein 5 \\
\hline ENSG00000140497 & 0.77 & SCAMP2 & Secretory carrier membrane protein 2 \\
\hline ENSG00000084754 & 0.77 & HADHA & $\begin{array}{l}\text { Hydroxyacyl-Coenzyme A dehydrogenase/3-ketoacyl- } \\
\text { Coenzyme A thiolase/enoyl-Coenzyme A hydratase } \\
\text { (trifunctional protein), alpha subunit }\end{array}$ \\
\hline ENSG00000132405 & 0.77 & TBC1D14 & TBC1 domain family, member 14 \\
\hline
\end{tabular}




\begin{tabular}{|c|c|c|c|}
\hline Ensemble ID & fc & GeneSymbol & Description \\
\hline ENSG00000198786 & 0.77 & MT-ND5 & NADH-ubiquinone oxidoreductase chain 5 \\
\hline ENSG00000149100 & 0.77 & PCID1 & B5 receptor \\
\hline ENSG00000006695 & 0.77 & COX10 & $\begin{array}{l}\text { COX10 homolog, cytochrome c oxidase assembly protein, } \\
\text { heme A: farnesyltransferase (yeast) }\end{array}$ \\
\hline ENSG00000136238 & 0.77 & RAC1 & $\begin{array}{l}\text { Ras-related C3 botulinum toxin substrate } 1 \text { (rho family, small } \\
\text { GTP binding protein Rac1) }\end{array}$ \\
\hline ENSG00000031698 & 0.77 & SARS & Seryl-tRNA synthetase \\
\hline ENSG00000179632 & 0.77 & MAF1 & MAF1 homolog (S. cerevisiae) \\
\hline ENSG00000157306 & 0.77 & THTPA & Thiamine triphosphatase \\
\hline ENSG00000058600 & 0.77 & POLR3E & Polymerase (RNA) III (DNA directed) polypeptide E (80kD) \\
\hline ENSG00000152642 & 0.77 & GPD1L & Glycerol-3-phosphate dehydrogenase 1-like \\
\hline ENSG00000185950 & 0.77 & IRS2 & Insulin receptor substrate 2 \\
\hline ENSG00000160917 & 0.77 & CPSF4 & Cleavage and polyadenylation specific factor $4,30 \mathrm{kDa}$ \\
\hline ENSG00000100902 & 0.77 & PSMA6 & Proteasome (prosome, macropain) subunit, alpha type, 6 \\
\hline ENSG00000124486 & 0.77 & USP9X & Ubiquitin specific peptidase 9, X-linked \\
\hline ENSG00000178952 & 0.77 & TUFM & Tu translation elongation factor, mitochondrial \\
\hline ENSG00000198771 & 0.77 & RCSD1 & RCSD domain containing 1 \\
\hline ENSG00000185262 & 0.77 & FAM100B & Family with sequence similarity 100 , member $B$ \\
\hline ENSG00000196792 & 0.77 & STRN3 & Striatin, calmodulin binding protein 3 \\
\hline ENSG00000101605 & 0.77 & MYOM1 & Myomesin 1, 185kDa \\
\hline ENSG00000175826 & 0.77 & DULLARD & Dullard homolog (Xenopus laevis) \\
\hline ENSG00000188021 & 0.77 & UBQLN2 & Ubiquilin 2 \\
\hline ENSG00000170310 & 0.77 & STX8 & Syntaxin 8 \\
\hline ENSG00000170881 & 0.77 & RNF139 & Ring finger protein 139 \\
\hline ENSG00000125821 & 0.77 & HARS2 & Histidyl-tRNA synthetase 2, mitochondrial (putative) \\
\hline ENSG00000168802 & 0.77 & Q71E72_HUMAN & $\begin{array}{l}\text { chromosome transmission fidelity factor } 8 \text { homolog }(\mathrm{S} . \\
\text { cerevisiae) }\end{array}$ \\
\hline ENSG00000129084 & 0.78 & PSMA1 & Proteasome (prosome, macropain) subunit, alpha type, 1 \\
\hline ENSG00000134775 & 0.78 & FHOD3 & Formin homology 2 domain containing 3 \\
\hline ENSG00000131446 & 0.78 & MGAT1 & $\begin{array}{l}\text { Mannosyl (alpha-1,3-)-glycoprotein beta-1,2-N- } \\
\text { acetylglucosaminyltransferase }\end{array}$ \\
\hline ENSG00000134014 & 0.78 & ELP3 & Elongation protein 3 homolog (S. cerevisiae) \\
\hline ENSG00000075568 & 0.78 & TMEM131 & Transmembrane protein 131 \\
\hline ENSG00000162923 & 0.78 & WDR26 & WD repeat domain 26 \\
\hline ENSG00000165704 & 0.78 & HPRT1 & Hypoxanthine phosphoribosyltransferase 1 \\
\hline ENSG00000151240 & 0.78 & DIP2C & DIP2 disco-interacting protein 2 homolog C (Drosophila) \\
\hline ENSG00000172765 & 0.78 & TMCC1 & Transmembrane and coiled-coil domain family 1 \\
\hline ENSG00000132388 & 0.78 & UBE2G1 & Ubiquitin-conjugating enzyme E2G 1 (UBC7 homolog, yeast) \\
\hline ENSG00000148834 & 0.78 & GSTO1 & Glutathione S-transferase omega 1 \\
\hline ENSG00000113360 & 0.78 & RNASEN & Ribonuclease type III, nuclear \\
\hline ENSG00000118564 & 0.78 & FBXL5 & F-box and leucine-rich repeat protein 5 \\
\hline ENSG00000171603 & 0.78 & CLSTN1 & Calsyntenin 1 \\
\hline ENSG00000178537 & 0.78 & SLC25A20 & $\begin{array}{l}\text { Solute carrier family } 25 \text { (carnitine/acylcarnitine translocase), } \\
\text { member } 20\end{array}$ \\
\hline ENSG00000168216 & 0.78 & LMBRD1 & LMBR1 domain containing 1 \\
\hline ENSG00000168884 & 0.78 & TNIP2 & TNFAIP3 interacting protein 2 \\
\hline ENSG00000111666 & 0.78 & CHPT1 & Choline phosphotransferase 1 \\
\hline
\end{tabular}




\begin{tabular}{|c|c|c|c|}
\hline Ensemble ID & fc & GeneSymbol & Description \\
\hline ENSG00000176871 & 0.78 & WSB2 & WD repeat and SOCS box-containing 2 \\
\hline ENSG00000064601 & 0.78 & CTSA & Cathepsin A \\
\hline ENSG00000146872 & 0.78 & TLK2 & Tousled-like kinase 2 \\
\hline ENSG00000154945 & 0.78 & ANKRD40 & Ankyrin repeat domain 40 \\
\hline ENSG00000180758 & 0.78 & GPR157 & G protein-coupled receptor 157 \\
\hline ENSG00000068120 & 0.78 & COASY & Coenzyme A synthase \\
\hline ENSG00000090615 & 0.78 & GOLGA3 & Golgi autoantigen, golgin subfamily a, 3 \\
\hline ENSG00000196507 & 0.78 & TCEAL3 & Transcription elongation factor A (SII)-like 3 \\
\hline ENSG00000197157 & 0.78 & SND1 & Staphylococcal nuclease and tudor domain containing 1 \\
\hline ENSG00000136758 & 0.78 & YME1L1 & YME1-like 1 (S. cerevisiae) \\
\hline ENSG00000158470 & 0.78 & B4GALT5 & $\begin{array}{l}\text { UDP-Gal:betaGlcNAc beta 1,4- galactosyltransferase, } \\
\text { polypeptide } 5\end{array}$ \\
\hline ENSG00000169083 & 0.78 & AR & Androgen receptor \\
\hline ENSG00000173692 & 0.78 & PSMD1 & $\begin{array}{l}\text { Proteasome (prosome, macropain) 26S subunit, non-ATPase, } \\
1\end{array}$ \\
\hline ENSG00000004776 & 0.78 & HSPB6 & Heat shock protein, alpha-crystallin-related, B6 \\
\hline ENSG00000013583 & 0.78 & HEBP1 & Heme binding protein 1 \\
\hline ENSG00000085377 & 0.78 & PREP & Prolyl endopeptidase \\
\hline ENSG00000105711 & 0.78 & SCN1B & Sodium channel, voltage-gated, type I, beta \\
\hline ENSG00000101457 & 0.78 & DNTTIP1 & Deoxynucleotidyltransferase, terminal, interacting protein 1 \\
\hline ENSG00000140350 & 0.78 & ANP32A & $\begin{array}{l}\text { Acidic (leucine-rich) nuclear phosphoprotein } 32 \text { family, member } \\
\text { A }\end{array}$ \\
\hline ENSG00000110958 & 0.78 & PTGES3 & Prostaglandin E synthase 3 (cytosolic) \\
\hline ENSG00000054793 & 0.78 & ATP9A & ATPase, class II, type 9A \\
\hline ENSG00000167674 & 0.79 & NP_001001520.1 & hepatoma-derived growth factor-related protein 2 isoform 1 \\
\hline ENSG00000126945 & 0.79 & HNRPH2 & Heterogeneous nuclear ribonucleoprotein $\mathrm{H}^{\prime}$ \\
\hline ENSG00000068903 & 0.79 & SIRT2 & $\begin{array}{l}\text { Sirtuin (silent mating type information regulation } 2 \text { homolog) } 2 \\
\text { (S. cerevisiae) }\end{array}$ \\
\hline ENSG00000186298 & 0.79 & PPP1CC & Protein phosphatase 1, catalytic subunit, gamma isoform \\
\hline ENSG00000061676 & 0.79 & NCKAP1 & NCK-associated protein 1 \\
\hline ENSG00000090621 & 0.79 & PABPC4 & Poly(A) binding protein, cytoplasmic 4 (inducible form) \\
\hline ENSG00000116871 & 0.79 & RPRC1 & arginine/proline rich coiled-coil 1 \\
\hline ENSG00000150459 & 0.79 & SAP18 & Sin3A-associated protein, $18 \mathrm{kDa}$ \\
\hline ENSG00000130021 & 0.79 & HDHD1A & Haloacid dehalogenase-like hydrolase domain containing $1 \mathrm{~A}$ \\
\hline ENSG00000197724 & 0.79 & PHF2 & PHD finger protein 2 \\
\hline ENSG00000088833 & 0.79 & NSFL1C & NSFL1 (p97) cofactor ( $p 47)$ \\
\hline ENSG00000148335 & 0.79 & C9orf32 & NA \\
\hline ENSG00000025800 & 0.79 & KPNA6 & Karyopherin alpha-6 \\
\hline ENSG00000121440 & 0.79 & PDZRN3 & PDZ domain containing ring finger 3 \\
\hline ENSG00000168734 & 0.79 & PKIG & Protein kinase (cAMP-dependent, catalytic) inhibitor gamma \\
\hline ENSG00000100288 & 0.79 & CPT1B & Carnitine palmitoyltransferase 1B (muscle) \\
\hline ENSG00000116096 & 0.79 & SPR & $\begin{array}{l}\text { Sepiapterin reductase (7,8-dihydrobiopterin:NADP+ } \\
\text { oxidoreductase) }\end{array}$ \\
\hline ENSG00000196455 & 0.79 & PIK3R4 & Phosphoinositide-3-kinase, regulatory subunit 4 \\
\hline ENSG00000204560 & 0.79 & DHX16 & DEAH (Asp-Glu-Ala-His) box polypeptide 16 \\
\hline ENSG00000116521 & 0.79 & SCAMP3 & Secretory carrier membrane protein 3 \\
\hline ENSG00000156026 & 0.79 & CCDC109A & Coiled-coil domain containing 109A \\
\hline
\end{tabular}




\begin{tabular}{|c|c|c|c|}
\hline Ensemble ID & fc & GeneSymbol & Description \\
\hline ENSG00000160967 & 0.79 & CUTL1 & NA \\
\hline ENSG00000101266 & 0.79 & CSNK2A1 & Casein kinase 2, alpha 1 polypeptide \\
\hline ENSG00000138629 & 0.79 & UBL7 & Ubiquitin-like 7 (bone marrow stromal cell-derived) \\
\hline ENSG00000028203 & 0.79 & VEZT & Vezatin, adherens junctions transmembrane protein \\
\hline ENSG00000108349 & 0.79 & CASC3 & Cancer susceptibility candidate 3 \\
\hline ENSG00000131378 & 0.79 & RFTN1 & Raftlin, lipid raft linker 1 \\
\hline ENSG00000149929 & 0.79 & HIRIP3 & HIRA interacting protein 3 \\
\hline ENSG00000146731 & 0.79 & CCT6A & Chaperonin containing TCP1, subunit $6 \mathrm{~A}$ (zeta 1) \\
\hline ENSG00000187715 & 0.79 & KLHDC6 & Kelch domain containing 6 \\
\hline ENSG00000071655 & 0.79 & MBD3 & Methyl-CpG binding domain protein 3 \\
\hline ENSG00000163346 & 0.79 & PBXIP1 & Pre-B-cell leukemia homeobox interacting protein 1 \\
\hline ENSG00000136478 & 0.79 & TEX2 & Testis expressed 2 \\
\hline ENSG00000006831 & 0.79 & ADIPOR2 & Adiponectin receptor 2 \\
\hline ENSG00000147416 & 0.79 & ATP6V1B2 & ATPase, $\mathrm{H}+$ transporting, lysosomal $56 / 58 \mathrm{kDa}, \mathrm{V} 1$ subunit $\mathrm{B} 2$ \\
\hline ENSG00000155506 & 0.79 & LARP1 & La ribonucleoprotein domain family, member 1 \\
\hline ENSG00000106290 & 0.79 & TAF6 & $\begin{array}{l}\text { TAF6 RNA polymerase II, TATA box binding protein (TBP)- } \\
\text { associated factor, } 80 \mathrm{kDa}\end{array}$ \\
\hline ENSG00000071205 & 0.79 & ARHGAP10 & Rho GTPase activating protein 10 \\
\hline ENSG00000121210 & 0.79 & KIAA0922 & KIAA0922 \\
\hline ENSG00000130175 & 0.79 & PRKCSH & Protein kinase $\mathrm{C}$ substrate $80 \mathrm{~K}-\mathrm{H}$ \\
\hline ENSG00000071462 & 0.79 & WBSCR22 & Williams Beuren syndrome chromosome region 22 \\
\hline ENSG00000171206 & 0.79 & TRIM8 & Tripartite motif-containing 8 \\
\hline ENSG00000174684 & 0.79 & B3GNT1 & $\begin{array}{l}\text { UDP-GIcNAc:betaGal beta-1,3-N- } \\
\text { acetylglucosaminyltransferase } 1\end{array}$ \\
\hline ENSG00000103942 & 0.79 & HOMER2 & Homer homolog 2 (Drosophila) \\
\hline ENSG00000130165 & 0.79 & ELOF1 & Elongation factor 1 homolog (S. cerevisiae) \\
\hline ENSG00000116649 & 0.79 & SRM & Spermidine synthase \\
\hline ENSG00000112276 & 0.79 & BVES & Blood vessel epicardial substance \\
\hline ENSG00000139405 & 0.79 & C12orf52 & Chromosome 12 open reading frame 52 \\
\hline ENSG00000004848 & 0.79 & ARX & Aristaless related homeobox \\
\hline ENSG00000143799 & 0.79 & PARP1 & Poly (ADP-ribose) polymerase 1 \\
\hline ENSG00000179041 & 0.79 & RRS1 & RRS1 ribosome biogenesis regulator homolog (S. cerevisiae) \\
\hline ENSG00000171307 & 0.79 & ZDHHC16 & Zinc finger, DHHC-type containing 16 \\
\hline ENSG00000155463 & 0.80 & OXA1L & Oxidase (cytochrome c) assembly 1-like \\
\hline ENSG00000142864 & 0.80 & SERBP1 & SERPINE1 mRNA binding protein 1 \\
\hline ENSG00000115685 & 0.80 & PPP1R7 & Protein phosphatase 1 , regulatory (inhibitor) subunit 7 \\
\hline ENSG00000079459 & 0.80 & FDFT1 & Farnesyl-diphosphate farnesyltransferase 1 \\
\hline ENSG00000067113 & 0.80 & PPAP2A & Phosphatidic acid phosphatase type $2 \mathrm{~A}$ \\
\hline ENSG00000165695 & 0.80 & C9orf98 & Chromosome 9 open reading frame 98 \\
\hline ENSG00000156599 & 0.80 & ZDHHC5 & Zinc finger, DHHC-type containing 5 \\
\hline ENSG00000122068 & 0.80 & FYTTD1 & Forty-two-three domain containing 1 \\
\hline ENSG00000131238 & 0.80 & PPT1 & Palmitoyl-protein thioesterase 1 \\
\hline ENSG00000149218 & 0.80 & ENDOD1 & Endonuclease domain containing 1 \\
\hline ENSG00000054523 & 0.80 & KIF1B & Kinesin family member $1 \mathrm{~B}$ \\
\hline
\end{tabular}




\begin{tabular}{|c|c|c|c|}
\hline Ensemble ID & fc & GeneSymbol & Description \\
\hline ENSG00000102178 & 0.80 & UBL4A & Ubiquitin-like 4A \\
\hline ENSG00000149547 & 0.80 & $\mathrm{El} 24$ & Etoposide induced 2.4 mRNA \\
\hline ENSG00000126581 & 0.80 & BECN1 & Beclin 1, autophagy related \\
\hline ENSG00000115241 & 0.80 & PPM1G & $\begin{array}{l}\text { Protein phosphatase } 1 \mathrm{G} \text { (formerly } 2 \mathrm{C} \text { ), magnesium-dependent, } \\
\text { gamma isoform }\end{array}$ \\
\hline ENSG00000069998 & 0.80 & CECR5 & Cat eye syndrome chromosome region, candidate 5 \\
\hline ENSG00000105202 & 0.80 & FBL & Fibrillarin \\
\hline ENSG00000079332 & 0.80 & SAR1A & SAR1 homolog A (S. cerevisiae) \\
\hline ENSG00000152795 & 0.80 & HNRPDL & Heterogeneous nuclear ribonucleoprotein D-like \\
\hline ENSG00000023734 & 0.80 & STRAP & Serine/threonine kinase receptor associated protein \\
\hline ENSG00000204155 & 0.80 & PARG & Poly(ADP-ribose) glycohydrolase \\
\hline ENSG00000053254 & 0.80 & CHES1 & Checkpoint suppressor 1 \\
\hline ENSG00000110048 & 0.80 & OSBP & Oxysterol binding protein \\
\hline ENSG00000111275 & 0.80 & ALDH2 & Aldehyde dehydrogenase 2 family (mitochondrial) \\
\hline ENSG00000140259 & 0.80 & MFAP1 & Microfibrillar-associated protein 1 \\
\hline ENSG00000174080 & 0.80 & CTSF & Cathepsin F \\
\hline ENSG00000108039 & 0.80 & XPNPEP1 & X-prolyl aminopeptidase (aminopeptidase P) 1, soluble \\
\hline ENSG00000187109 & 0.80 & NAP1L1 & Nucleosome assembly protein 1 -like 1 \\
\hline ENSG00000148541 & 0.80 & FAM13C1 & Protein FAM13C1 \\
\hline ENSG00000152443 & 0.80 & ZNF776 & Zinc finger protein 776 \\
\hline ENSG00000173933 & 0.80 & RBM4 & RNA binding motif protein 4 \\
\hline ENSG00000143384 & 0.80 & MCL1 & Myeloid cell leukemia sequence 1 (BCL2-related) \\
\hline ENSG00000138449 & 0.80 & SLC40A1 & Solute carrier family 40 (iron-regulated transporter), member 1 \\
\hline ENSG00000113643 & 0.80 & RARS & Arginyl-tRNA synthetase \\
\hline ENSG00000077157 & 0.80 & PPP1R12B & Protein phosphatase 1 , regulatory (inhibitor) subunit 12B \\
\hline ENSG00000111332 & 0.80 & H2AFJ & $\mathrm{H} 2 \mathrm{~A}$ histone family, member $\mathrm{J}$ isoform 2 \\
\hline ENSG00000105393 & 0.80 & NP_001028721.1 & NA \\
\hline ENSG00000143771 & 0.80 & $\mathrm{CNIH} 4$ & Cornichon homolog 4 (Drosophila) \\
\hline ENSG00000183624 & 0.80 & C3orf37 & Chromosome 3 open reading frame 37 \\
\hline ENSG00000112478 & 0.80 & VPS52 & Vacuolar protein sorting 52 homolog (S. cerevisiae) \\
\hline ENSG00000140543 & 0.80 & DET1 & De-etiolated homolog 1 (Arabidopsis) \\
\hline ENSG00000109534 & 0.80 & NOLA1 & Nucleolar protein family A member 1 \\
\hline ENSG00000136205 & 0.80 & TNS3 & Tensin 3 \\
\hline ENSG00000147065 & 0.80 & MSN & Moesin \\
\hline ENSG00000122359 & 0.80 & ANXA11 & Annexin A11 \\
\hline ENSG00000196704 & 0.80 & AMZ2_HUMAN & Archaemetzincin-2 \\
\hline ENSG00000167136 & 0.80 & ENDOG & Endonuclease G \\
\hline ENSG00000151929 & 0.80 & BAG3 & BCL2-associated athanogene 3 \\
\hline ENSG00000157600 & 0.80 & TMEM164 & Transmembrane protein 164 \\
\hline ENSG00000077348 & 0.80 & EXOSC5 & Exosome component 5 \\
\hline ENSG00000187239 & 0.80 & FNBP1 & Formin binding protein 1 \\
\hline ENSG00000110651 & 0.80 & CD81 & CD81 molecule \\
\hline ENSG00000119280 & 0.80 & C1orf198 & Chromosome 1 open reading frame 198 \\
\hline ENSG00000166272 & 0.80 & C10orf26 & NA \\
\hline
\end{tabular}




\begin{tabular}{|c|c|c|c|}
\hline Ensemble ID & fc & GeneSymbol & Description \\
\hline ENSG00000097033 & 0.80 & SH3GLB1 & SH3-domain GRB2-like endophilin B1 \\
\hline ENSG00000110046 & 0.80 & NP_055919.1 & NA \\
\hline ENSG00000119718 & 0.80 & EIF2B2 & Eukaryotic translation initiation factor $2 \mathrm{~B}$, subunit 2 beta, $39 \mathrm{kDa}$ \\
\hline ENSG00000089289 & 0.80 & IGBP1 & Immunoglobulin (CD79A) binding protein 1 \\
\hline ENSG00000125835 & 0.80 & SNRPB & Small nuclear ribonucleoprotein polypeptides B and B1 \\
\hline ENSG00000172939 & 0.81 & OXSR1 & Oxidative-stress responsive 1 \\
\hline ENSG00000104872 & 0.81 & NOP17 & NA \\
\hline ENSG00000114786 & 0.81 & ACY1 & Aminoacylase 1 \\
\hline ENSG00000024422 & 0.81 & EHD2 & EH-domain containing 2 \\
\hline ENSG00000211861 & 0.81 & TRAJ28 & T-cell receptor alpha $\mathrm{J}$ gene segment \\
\hline ENSG00000022277 & 0.81 & C20orf43 & Chromosome 20 open reading frame 43 \\
\hline ENSG00000069275 & 0.81 & NUCKS1 & $\begin{array}{l}\text { Nuclear ubiquitous casein and cyclin-dependent kinases } \\
\text { substrate }\end{array}$ \\
\hline ENSG00000088256 & 0.81 & GNA11 & Guanine nucleotide-binding protein subunit alpha-11 \\
\hline ENSG00000106993 & 0.81 & CDC37L1 & Cell division cycle 37 homolog (S. cerevisiae)-like 1 \\
\hline ENSG00000102978 & 0.81 & POLR2C & Polymerase (RNA) II (DNA directed) polypeptide C, 33kDa \\
\hline ENSG00000067560 & 0.81 & $\mathrm{RHOA}$ & Ras homolog gene family, member A \\
\hline ENSG00000108592 & 0.81 & FTSJ3 & FtsJ homolog 3 (E. coli) \\
\hline ENSG00000186660 & 0.81 & ZFP91 & Zinc finger protein 91 homolog (mouse) \\
\hline ENSG00000102226 & 0.81 & USP11 & Ubiquitin specific peptidase 11 \\
\hline ENSG00000135049 & 0.81 & AGTPBP1 & ATP/GTP binding protein 1 \\
\hline ENSG00000113552 & 0.81 & GNPDA1 & Glucosamine-6-phosphate deaminase 1 \\
\hline ENSG00000175203 & 0.81 & DCTN2 & Dynactin 2 (p50) \\
\hline ENSG00000088543 & 0.81 & C3orf18 & Chromosome 3 open reading frame 18 \\
\hline ENSG00000198836 & 0.81 & OPA1 & Optic atrophy 1 (autosomal dominant) \\
\hline ENSG00000129691 & 0.81 & ASH2L & Ash2 (absent, small, or homeotic)-like (Drosophila) \\
\hline ENSG00000137492 & 0.81 & PRKRIR & $\begin{array}{l}\text { Protein-kinase, interferon-inducible double stranded RNA } \\
\text { dependent inhibitor, repressor of (P58 repressor) }\end{array}$ \\
\hline ENSG00000135211 & 0.81 & TMEM60 & Transmembrane protein 60 \\
\hline ENSG00000047849 & 0.81 & MAP4 & Microtubule-associated protein 4 \\
\hline ENSG00000198492 & 0.81 & YTHDF2 & YTH domain family, member 2 \\
\hline ENSG00000138107 & 0.81 & ACTR1A & $\begin{array}{l}\text { ARP1 actin-related protein } 1 \text { homolog A, centractin alpha } \\
\text { (yeast) }\end{array}$ \\
\hline ENSG00000130826 & 0.81 & DKC1 & Dyskeratosis congenita 1 , dyskerin \\
\hline ENSG00000065427 & 0.81 & KARS & Lysyl-tRNA synthetase \\
\hline ENSG00000057608 & 0.81 & GDI2 & GDP dissociation inhibitor 2 \\
\hline ENSG00000066044 & 0.81 & ELAVL1 & $\begin{array}{l}\text { ELAV (embryonic lethal, abnormal vision, Drosophila)-like } 1 \text { (Hu } \\
\text { antigen R) }\end{array}$ \\
\hline ENSG00000121073 & 0.81 & SLC35B1 & Solute carrier family 35, member B1 \\
\hline ENSG00000138430 & 0.81 & GTPBP9 & Putative GTP-binding protein 9 \\
\hline ENSG00000102893 & 0.81 & PHKB & Phosphorylase kinase, beta \\
\hline ENSG00000135452 & 0.81 & TSPAN31 & Tetraspanin 31 \\
\hline ENSG00000141905 & 0.81 & NFIC & Nuclear factor I/C (CCAAT-binding transcription factor) \\
\hline ENSG00000116954 & 0.81 & RRAGC & Ras-related GTP binding C \\
\hline ENSG00000070770 & 0.81 & CSNK2A2 & Casein kinase 2, alpha prime polypeptide \\
\hline ENSG00000156253 & 0.81 & C21orf6 & NA \\
\hline
\end{tabular}




\begin{tabular}{|c|c|c|c|}
\hline Ensemble ID & fc & GeneSymbol & Description \\
\hline ENSG00000115053 & 0.81 & NCL & Nucleolin \\
\hline ENSG00000089154 & 0.81 & GCN1L1 & GCN1 general control of amino-acid synthesis 1 -like 1 (yeast) \\
\hline ENSG00000147649 & 0.81 & MTDH & Metadherin \\
\hline ENSG00000073111 & 0.81 & MCM2 & Minichromosome maintenance complex component 2 \\
\hline ENSG00000162735 & 0.81 & PEX19 & Peroxisomal biogenesis factor 19 \\
\hline ENSG00000111144 & 0.81 & LTA4H & Leukotriene A4 hydrolase \\
\hline ENSG00000115561 & 0.81 & VPS24 & Vacuolar protein sorting 24 homolog (S. cerevisiae) \\
\hline ENSG00000183576 & 0.81 & SETD3 & SET domain containing 3 \\
\hline ENSG00000184047 & 0.81 & DIABLO & Diablo homolog (Drosophila) \\
\hline ENSG00000185624 & 0.81 & $\mathrm{P} 4 \mathrm{HB}$ & Prolyl 4-hydroxylase, beta polypeptide \\
\hline ENSG00000116748 & 0.81 & AMPD1 & Adenosine monophosphate deaminase 1 (isoform M) \\
\hline ENSG00000152700 & 0.81 & SAR1B & SAR1 homolog B (S. cerevisiae) \\
\hline ENSG00000165678 & 0.81 & GHITM & Growth hormone inducible transmembrane protein \\
\hline ENSG00000198853 & 0.81 & RUSC2 & $\mathrm{RUN}$ and $\mathrm{SH} 3$ domain containing 2 \\
\hline ENSG00000078808 & 0.81 & SDF4 & Stromal cell derived factor 4 \\
\hline ENSG00000169446 & 0.82 & TMEM32 & transmembrane protein 32 \\
\hline ENSG00000161016 & 0.82 & RPL8 & Ribosomal protein L8 \\
\hline ENSG00000168286 & 0.82 & THAP11 & THAP domain containing 11 \\
\hline ENSG00000107341 & 0.82 & UBE2R2 & Ubiquitin-conjugating enzyme E2R 2 \\
\hline ENSG00000204564 & 0.82 & C6orf136 & Chromosome 6 open reading frame 136 \\
\hline ENSG00000132768 & 0.82 & DPH2 & DPH2 homolog (S. cerevisiae) \\
\hline ENSG00000101126 & 0.82 & ADNP & Activity-dependent neuroprotector homeobox \\
\hline ENSG00000100804 & 0.82 & PSMB5 & Proteasome (prosome, macropain) subunit, beta type, 5 \\
\hline ENSG00000151208 & 0.82 & DLG5 & Discs, large homolog 5 (Drosophila) \\
\hline ENSG00000142687 & 0.82 & KIAA0319L & KIAA0319-like \\
\hline ENSG00000167325 & 0.82 & RRM1 & Ribonucleotide reductase $\mathrm{M} 1$ \\
\hline ENSG00000073905 & 0.82 & VDAC1 & Voltage-dependent anion channel 1 \\
\hline ENSG00000168329 & 0.82 & CX3CR1 & Chemokine (C-X3-C motif) receptor 1 \\
\hline ENSG00000039123 & 0.82 & SKIV2L2 & Superkiller viralicidic activity 2-like 2 (S. cerevisiae) \\
\hline ENSG00000100811 & 0.82 & YY1 & YY1 transcription factor \\
\hline ENSG00000183207 & 0.82 & RUVBL2 & RuvB-like 2 (E. coli) \\
\hline ENSG00000116273 & 0.82 & PHF13 & PHD finger protein 13 \\
\hline ENSG00000124201 & 0.82 & ZNFX1 & Zinc finger, NFX1-type containing 1 \\
\hline ENSG00000126778 & 0.82 & SIX1 & SIX homeobox 1 \\
\hline ENSG00000103544 & 0.82 & NP_064710.3 & esophageal cancer associated protein (MGC16824) \\
\hline ENSG00000086232 & 0.82 & EIF2AK1 & Eukaryotic translation initiation factor 2-alpha kinase 1 \\
\hline ENSG00000160570 & 0.82 & DEDD2 & Death effector domain containing 2 \\
\hline ENSG00000042022 & 0.82 & ABHD14A & Abhydrolase domain containing $14 \mathrm{~A}$ \\
\hline ENSG00000062582 & 0.82 & MRPS24 & Mitochondrial ribosomal protein S24 \\
\hline ENSG00000101444 & 0.82 & $\mathrm{AHCY}$ & S-adenosylhomocysteine hydrolase \\
\hline ENSG00000198467 & 0.82 & TPM2 & Tropomyosin 2 (beta) \\
\hline ENSG00000101367 & 0.82 & MAPRE1 & Microtubule-associated protein, RP/EB family, member 1 \\
\hline ENSG00000142784 & 0.82 & WDTC1 & WD and tetratricopeptide repeats 1 \\
\hline
\end{tabular}




\begin{tabular}{|c|c|c|c|}
\hline Ensemble ID & fc & GeneSymbol & Description \\
\hline ENSG00000184900 & 0.82 & SUMO3 & SMT3 suppressor of mif two 3 homolog 3 (S. cerevisiae) \\
\hline ENSG00000129351 & 0.82 & ILF3 & Interleukin enhancer binding factor $3,90 \mathrm{kDa}$ \\
\hline ENSG00000151465 & 0.82 & CDC123 & Cell division cycle 123 homolog (S. cerevisiae) \\
\hline ENSG00000185885 & 0.82 & IFITM1 & Interferon induced transmembrane protein $1(9-27)$ \\
\hline ENSG00000099203 & 0.82 & TMED1 & Transmembrane emp24 protein transport domain containing 1 \\
\hline ENSG00000067182 & 0.82 & TNFRSF1A & Tumor necrosis factor receptor superfamily, member $1 \mathrm{~A}$ \\
\hline ENSG00000187147 & 0.82 & C1orf164 & NA \\
\hline ENSG00000161714 & 0.82 & PLCD3 & Phospholipase C, delta 3 \\
\hline ENSG00000124789 & 0.82 & NUP153 & Nuclear pore complex protein Nup153 \\
\hline ENSG00000164168 & 0.82 & TMEM34 & transmembrane protein 34 \\
\hline ENSG00000165424 & 0.82 & C10orf56 & NA \\
\hline ENSG00000203879 & 0.82 & GDI1 & GDP dissociation inhibitor 1 \\
\hline ENSG00000148672 & 0.82 & GLUD1 & Glutamate dehydrogenase 1 \\
\hline ENSG00000038274 & 0.82 & MAT2B & Methionine adenosyltransferase II, beta \\
\hline ENSG00000204580 & 0.82 & DDR1 & Discoidin domain receptor tyrosine kinase 1 \\
\hline ENSG00000105220 & 0.82 & GPI & Glucose phosphate isomerase \\
\hline ENSG00000132912 & 0.82 & DCTN4 & Dynactin 4 (p62) \\
\hline ENSG00000184113 & 0.82 & CLDN5 & Claudin 5 \\
\hline ENSG00000156976 & 0.82 & EIF4A2 & Eukaryotic initiation factor $4 \mathrm{~A}-\mathrm{II}$ \\
\hline ENSG00000180329 & 0.82 & CCDC43 & Coiled-coil domain containing 43 \\
\hline ENSG00000177666 & 0.82 & PNPLA2 & Patatin-like phospholipase domain containing 2 \\
\hline ENSG00000030582 & 0.82 & GRN & Granulin \\
\hline ENSG00000197894 & 0.82 & ADH5 & Alcohol dehydrogenase 5 (class III), chi polypeptide \\
\hline ENSG00000066027 & 0.83 & PPP2R5A & Protein phosphatase 2 , regulatory subunit B', alpha isoform \\
\hline ENSG00000160679 & 0.83 & C1orf77 & Chromosome 1 open reading frame 77 \\
\hline ENSG00000173641 & 0.83 & HSPB7 & Heat shock 27kDa protein family, member 7 (cardiovascular) \\
\hline ENSG00000124104 & 0.83 & SNX21 & Sorting nexin family member 21 \\
\hline ENSG00000125967 & 0.83 & APBA2BP & $\begin{array}{l}\text { Amyloid beta A4 protein-binding family A member 2-binding } \\
\text { protein }\end{array}$ \\
\hline ENSG00000103266 & 0.83 & STUB1 & STIP1 homology and U-box containing protein 1 \\
\hline ENSG00000143815 & 0.83 & LBR & Lamin B receptor \\
\hline ENSG00000112855 & 0.83 & HARSL & Probable histidyl-tRNA synthetase \\
\hline ENSG00000115415 & 0.83 & STAT1 & Signal transducer and activator of transcription $1,91 \mathrm{kDa}$ \\
\hline ENSG00000072506 & 0.83 & HSD17B10 & Hydroxysteroid (17-beta) dehydrogenase 10 \\
\hline ENSG00000138029 & 0.83 & HADHB & $\begin{array}{l}\text { Hydroxyacyl-Coenzyme A dehydrogenase/3-ketoacyl- } \\
\text { Coenzyme A thiolase/enoyl-Coenzyme A hydratase } \\
\text { (trifunctional protein), beta subunit }\end{array}$ \\
\hline ENSG00000136715 & 0.83 & SAP130 & Sin $3 \mathrm{~A}$-associated protein, $130 \mathrm{kDa}$ \\
\hline ENSG00000130706 & 0.83 & ADRM1 & Adhesion regulating molecule 1 \\
\hline ENSG00000131508 & 0.83 & UBE2D2 & Ubiquitin-conjugating enzyme E2D 2 (UBC4/5 homolog, yeast) \\
\hline ENSG00000142444 & 0.83 & C19orf52 & Chromosome 19 open reading frame 52 \\
\hline ENSG00000164896 & 0.83 & FASTK & Fas-activated serine/threonine kinase \\
\hline ENSG00000116688 & 0.83 & MFN2 & Mitofusin 2 \\
\hline ENSG00000156261 & 0.83 & CCT8 & Chaperonin containing TCP1, subunit 8 (theta) \\
\hline ENSG00000136240 & 0.83 & KDELR2 & KDEL (Lys-Asp-Glu-Leu) endoplasmic reticulum protein \\
\hline
\end{tabular}




\begin{tabular}{|c|c|c|c|}
\hline Ensemble ID & fc & GeneSymbol & Description \\
\hline & & & retention receptor 2 \\
\hline ENSG00000169032 & 0.83 & MAP2K1 & Mitogen-activated protein kinase kinase 1 \\
\hline ENSG00000173357 & 0.83 & Q13383_HUMAN & RNA binding motif (Fragment) \\
\hline ENSG00000075945 & 0.83 & KIFAP3 & Kinesin-associated protein 3 \\
\hline ENSG00000080503 & 0.83 & SMARCA2 & $\begin{array}{l}\text { SWI/SNF related, matrix associated, actin dependent regulator } \\
\text { of chromatin, subfamily a, member } 2\end{array}$ \\
\hline ENSG00000188549 & 0.83 & NP_997263.1 & NA \\
\hline ENSG00000085511 & 0.83 & MAP3K4 & Mitogen-activated protein kinase kinase kinase 4 \\
\hline ENSG00000116473 & 0.83 & RAP1A & RAP1A, member of RAS oncogene family \\
\hline ENSG00000172775 & 0.83 & NIP30_HUMAN & NEFA-interacting nuclear protein NIP30 \\
\hline ENSG00000135862 & 0.83 & LAMC1 & Laminin, gamma 1 (formerly LAMB2) \\
\hline ENSG00000197006 & 0.83 & METTL9 & Methyltransferase like 9 \\
\hline ENSG00000179010 & 0.83 & MRFAP1 & Mof4 family associated protein 1 \\
\hline ENSG00000130511 & 0.83 & SSBP4 & Single stranded DNA binding protein 4 \\
\hline ENSG00000077721 & 0.83 & UBE2A & Ubiquitin-conjugating enzyme E2A (RAD6 homolog) \\
\hline ENSG00000107796 & 0.83 & ACTA2 & Actin, alpha 2, smooth muscle, aorta \\
\hline ENSG00000154153 & 0.83 & NP_001030022.1 & NA \\
\hline ENSG00000104442 & 0.83 & ARMC1 & Armadillo repeat containing 1 \\
\hline ENSG00000101421 & 0.83 & CHMP4B & Chromatin modifying protein 4B \\
\hline ENSG00000205352 & 0.83 & NP_001005354.1 & proline rich 13 isoform 2 \\
\hline ENSG00000134440 & 0.83 & NARS & Asparaginyl-tRNA synthetase \\
\hline ENSG00000168090 & 0.83 & COPS6 & $\begin{array}{l}\text { COP9 constitutive photomorphogenic homolog subunit } 6 \\
\text { (Arabidopsis) }\end{array}$ \\
\hline ENSG00000155876 & 0.83 & RRAGA & Ras-related GTP binding A \\
\hline ENSG00000100395 & 0.83 & L3MBTL2 & L(3)mbt-like 2 (Drosophila) \\
\hline ENSG00000122687 & 0.83 & FTSJ2 & FtsJ homolog 2 (E. coli) \\
\hline ENSG00000120265 & 0.83 & PCMT1 & Protein-L-isoaspartate (D-aspartate) O-methyltransferase \\
\hline ENSG00000065150 & 0.83 & RANBP5 & Ran-binding protein 5 \\
\hline ENSG00000131773 & 0.84 & KHDRBS3 & $\begin{array}{l}\mathrm{KH} \text { domain containing, RNA binding, signal transduction } \\
\text { associated } 3\end{array}$ \\
\hline ENSG00000119632 & 0.84 & FAM14A & Protein FAM14A precursor \\
\hline ENSG00000078674 & 0.84 & PCM1 & Pericentriolar material 1 \\
\hline ENSG00000039523 & 0.84 & FAM65A & Family with sequence similarity 65 , member $A$ \\
\hline ENSG00000115234 & 0.84 & SNX17 & Sorting nexin 17 \\
\hline ENSG00000135636 & 0.84 & DYSF & $\begin{array}{l}\text { Dysferlin, limb girdle muscular dystrophy 2B (autosomal } \\
\text { recessive) }\end{array}$ \\
\hline ENSG00000102054 & 0.84 & RBBP7 & Retinoblastoma binding protein 7 \\
\hline ENSG00000171720 & 0.84 & HDAC3 & Histone deacetylase 3 \\
\hline ENSG00000108799 & 0.84 & $\mathrm{EZH} 1$ & Enhancer of zeste homolog 1 (Drosophila) \\
\hline ENSG00000114686 & 0.84 & MRPL3 & Mitochondrial ribosomal protein L3 \\
\hline ENSG00000115548 & 0.84 & JMJD1A & JmjC domain-containing histone demethylation protein $2 \mathrm{~A}$ \\
\hline ENSG00000168175 & 0.84 & C14orf32 & NA \\
\hline ENSG00000189311 & 0.84 & PPME1 & Protein phosphatase methylesterase 1 \\
\hline ENSG00000050426 & 0.84 & LETMD1 & LETM1 domain containing 1 \\
\hline ENSG00000122042 & 0.84 & UBL3 & Ubiquitin-like 3 \\
\hline ENSG00000129170 & 0.84 & CSRP3 & Cysteine and glycine-rich protein 3 (cardiac LIM protein) \\
\hline
\end{tabular}




\begin{tabular}{|c|c|c|c|}
\hline Ensemble ID & fc & GeneSymbol & Description \\
\hline ENSG00000132581 & 0.84 & SDF2 & Stromal cell-derived factor 2 \\
\hline ENSG00000204427 & 0.84 & BAT5 & HLA-B associated transcript 5 \\
\hline ENSG00000120705 & 0.84 & ETF1 & Eukaryotic translation termination factor 1 \\
\hline ENSG00000196591 & 0.84 & HDAC2 & Histone deacetylase 2 \\
\hline ENSG00000064545 & 0.84 & TMEM161A & Transmembrane protein $161 \mathrm{~A}$ \\
\hline ENSG00000071051 & 0.84 & NCK2 & NCK adaptor protein 2 \\
\hline ENSG00000117748 & 0.84 & RPA2 & Replication protein $\mathrm{A} 2,32 \mathrm{kDa}$ \\
\hline ENSG00000023697 & 0.84 & DERA & 2-deoxyribose-5-phosphate aldolase homolog (C. elegans) \\
\hline ENSG00000048392 & 0.84 & RRM2B & Ribonucleotide reductase M2 B (TP53 inducible) \\
\hline ENSG00000011523 & 0.84 & CEP68 & Centrosomal protein $68 \mathrm{kDa}$ \\
\hline ENSG00000198218 & 0.84 & QRICH1 & Glutamine-rich 1 \\
\hline ENSG00000108671 & 0.84 & PSMD11 & $\begin{array}{l}\text { Proteasome (prosome, macropain) } 26 \mathrm{~S} \text { subunit, non-ATPase, } \\
11\end{array}$ \\
\hline ENSG00000196781 & 0.84 & TLE1 & Transducin-like enhancer protein 1 \\
\hline ENSG00000104870 & 0.84 & FCGRT & Fc fragment of IgG, receptor, transporter, alpha \\
\hline ENSG00000075413 & 0.84 & MARK3 & $\mathrm{MAP} /$ microtubule affinity-regulating kinase 3 \\
\hline ENSG00000153904 & 0.84 & $\mathrm{DDAH} 1$ & Dimethylarginine dimethylaminohydrolase 1 \\
\hline ENSG00000107438 & 0.84 & PDLIM1 & PDZ and LIM domain 1 \\
\hline ENSG00000135617 & 0.84 & C2orf7 & Chromosome 2 open reading frame 7 \\
\hline ENSG00000084623 & 0.84 & EIF3S2 & Eukaryotic translation initiation factor 3 subunit 2 \\
\hline ENSG00000198961 & 0.84 & PJA2 & Praja ring finger 2 \\
\hline ENSG00000181709 & 0.84 & Q96DH5_HUMAN & GNA11 protein \\
\hline ENSG00000091164 & 0.84 & TXNL1 & Thioredoxin-like 1 \\
\hline ENSG00000156298 & 0.84 & TSPAN7 & Tetraspanin 7 \\
\hline ENSG00000137100 & 0.84 & DCTN3 & Dynactin 3 (p22) \\
\hline ENSG00000085063 & 0.84 & CD59 & CD59 glycoprotein precursor \\
\hline ENSG00000132688 & 0.84 & NES & Nestin \\
\hline ENSG00000134058 & 0.84 & CDK7 & Cyclin-dependent kinase 7 \\
\hline ENSG00000102030 & 0.84 & TRIM23 & Tripartite motif-containing 23 \\
\hline ENSG00000037474 & 0.84 & NSUN2 & NOL1/NOP2/Sun domain family, member 2 \\
\hline ENSG00000110696 & 0.84 & C11orf58 & Chromosome 11 open reading frame 58 \\
\hline ENSG00000125772 & 0.84 & GDE5_HUMAN & Putative glycerophosphodiester phosphodiesterase 5 \\
\hline ENSG00000166619 & 0.84 & BLCAP & Bladder cancer associated protein \\
\hline ENSG00000090487 & 0.84 & SPG21 & Spastic paraplegia 21 (autosomal recessive, Mast syndrome) \\
\hline ENSG00000113648 & 0.84 & H2AFY & Core histone macro-H2A.1 \\
\hline ENSG00000185630 & 0.84 & PBX1 & Pre-B-cell leukemia homeobox 1 \\
\hline ENSG00000182551 & 0.85 & ADI1 & Acireductone dioxygenase 1 \\
\hline ENSG00000163344 & 0.85 & PMVK & Phosphomevalonate kinase \\
\hline ENSG00000168264 & 0.85 & IRF2BP2 & Interferon regulatory factor 2 binding protein 2 \\
\hline ENSG00000188725 & 0.85 & NP_001041714.1 & NA \\
\hline ENSG00000100083 & 0.85 & GGA1 & $\begin{array}{l}\text { Golgi associated, gamma adaptin ear containing, ARF binding } \\
\text { protein } 1\end{array}$ \\
\hline ENSG00000109919 & 0.85 & MTCH2 & Mitochondrial carrier homolog 2 (C. elegans) \\
\hline ENSG00000137815 & 0.85 & RTF1 & $\begin{array}{l}\text { Rtf1, Paf1/RNA polymerase II complex component, homolog } \\
\text { (S. cerevisiae) }\end{array}$ \\
\hline
\end{tabular}




\begin{tabular}{|c|c|c|c|}
\hline Ensemble ID & fc & GeneSymbol & Description \\
\hline ENSG00000166439 & 0.85 & RNF169 & Ring finger protein 169 \\
\hline ENSG00000125733 & 0.85 & TRIP10 & Thyroid hormone receptor interactor 10 \\
\hline ENSG00000136842 & 0.85 & TMOD1 & Tropomodulin 1 \\
\hline ENSG00000130638 & 0.85 & ATXN10 & Ataxin 10 \\
\hline ENSG00000065183 & 0.85 & WDR3 & WD repeat domain 3 \\
\hline ENSG00000158864 & 0.85 & NDUFS2 & $\begin{array}{l}\text { NADH dehydrogenase (ubiquinone) Fe-S protein } 2,49 \mathrm{kDa} \\
\text { (NADH-coenzyme } \mathrm{Q} \text { reductase) }\end{array}$ \\
\hline ENSG00000092330 & 0.85 & TINF2 & TERF1 (TRF1)-interacting nuclear factor 2 \\
\hline ENSG00000188895 & 0.85 & Q68DK7_HUMAN & MSL-1 protein \\
\hline ENSG00000141627 & 0.85 & DYM & Dymeclin \\
\hline ENSG00000135316 & 0.85 & SYNCRIP & Synaptotagmin binding, cytoplasmic RNA interacting protein \\
\hline ENSG00000140400 & 0.85 & MAN2C1 & Alpha-mannosidase $2 \mathrm{C} 1$ \\
\hline ENSG00000091436 & 0.85 & MLTK_HUMAN & Mitogen-activated protein kinase kinase kinase \\
\hline ENSG00000105186 & 0.85 & ANKRD27 & Ankyrin repeat domain 27 (VPS9 domain) \\
\hline ENSG00000171914 & 0.85 & TLN2 & Talin 2 \\
\hline ENSG00000115993 & 0.85 & TRAK2 & Trafficking protein, kinesin binding 2 \\
\hline ENSG00000149658 & 0.85 & YTHDF1 & YTH domain family, member 1 \\
\hline ENSG00000106397 & 0.85 & PLOD3 & Procollagen-lysine, 2-oxoglutarate 5-dioxygenase 3 \\
\hline ENSG00000174282 & 0.85 & ZBTB4 & Zinc finger and BTB domain containing 4 \\
\hline ENSG00000180902 & 0.85 & $\mathrm{D} 2 \mathrm{HGDH}$ & D-2-hydroxyglutarate dehydrogenase \\
\hline ENSG00000126746 & 0.85 & ZNF384 & Zinc finger protein 384 \\
\hline ENSG00000140750 & 0.85 & ARHGAP17 & Rho GTPase activating protein 17 \\
\hline ENSG00000100031 & 0.85 & GGT1 & Gamma-glutamyltransferase 1 \\
\hline ENSG00000157823 & 0.85 & AP3S2 & Adaptor-related protein complex 3 , sigma 2 subunit \\
\hline ENSG00000130741 & 0.85 & EIF2S3 & $\begin{array}{l}\text { Eukaryotic translation initiation factor } 2 \text {, subunit } 3 \text { gamma, } \\
52 \mathrm{kDa}\end{array}$ \\
\hline ENSG00000105497 & 0.85 & ZNF175 & Zinc finger protein 175 \\
\hline ENSG00000149792 & 0.85 & MRPL49 & Mitochondrial ribosomal protein L49 \\
\hline ENSG00000143401 & 0.85 & ANP32E & $\begin{array}{l}\text { Acidic (leucine-rich) nuclear phosphoprotein } 32 \text { family, member } \\
\text { E }\end{array}$ \\
\hline ENSG00000122779 & 0.85 & TRIM24 & Tripartite motif-containing 24 \\
\hline ENSG00000067365 & 0.85 & C16orf68 & Chromosome 16 open reading frame 68 \\
\hline ENSG00000114784 & 0.85 & EIF1B & Eukaryotic translation initiation factor $1 \mathrm{~B}$ \\
\hline ENSG00000167705 & 0.85 & RILP & Rab interacting lysosomal protein \\
\hline ENSG00000104980 & 0.85 & TIMM44 & $\begin{array}{l}\text { Translocase of inner mitochondrial membrane } 44 \text { homolog } \\
\text { (yeast) }\end{array}$ \\
\hline ENSG00000138081 & 0.85 & FBXO11 & F-box protein 11 \\
\hline ENSG00000099804 & 0.85 & CDC34 & Cell division cycle 34 homolog (S. cerevisiae) \\
\hline ENSG00000165119 & 0.85 & HNRPK & Heterogeneous nuclear ribonucleoprotein $\mathrm{K}$ \\
\hline ENSG00000177963 & 0.85 & RIC8A & $\begin{array}{l}\text { Resistance to inhibitors of cholinesterase } 8 \text { homolog A (C. } \\
\text { elegans) }\end{array}$ \\
\hline ENSG00000087269 & 0.85 & C4orf9 & NA \\
\hline ENSG00000188026 & 0.85 & NP_847884.1 & NA \\
\hline ENSG00000055211 & 0.85 & C6orf72 & Chromosome 6 open reading frame 72 \\
\hline ENSG00000132153 & 0.85 & $\mathrm{DHX30}$ & DEAH (Asp-Glu-Ala-His) box polypeptide 30 \\
\hline ENSG00000114480 & 0.85 & GBE1 & Glucan (1,4-alpha-), branching enzyme 1 \\
\hline ENSG00000134108 & 0.85 & ARL8B & ADP-ribosylation factor-like $8 \mathrm{~B}$ \\
\hline
\end{tabular}




\begin{tabular}{|c|c|c|c|}
\hline Ensemble ID & fc & GeneSymbol & Description \\
\hline ENSG00000112320 & 0.85 & NP_060483.3 & NA \\
\hline ENSG00000084073 & 0.85 & ZMPSTE24 & Zinc metallopeptidase (STE24 homolog, S. cerevisiae) \\
\hline ENSG00000119446 & 0.85 & RBM18 & RNA binding motif protein 18 \\
\hline ENSG00000148143 & 0.85 & ZNF462 & Zinc finger protein 462 \\
\hline ENSG00000138095 & 0.85 & LRPPRC & Leucine-rich PPR-motif containing \\
\hline ENSG00000161999 & 0.85 & NP_001005920.2 & Similar to C. Elegans protein F17C8.5 \\
\hline ENSG00000131779 & 0.85 & PEX11B & Peroxisomal biogenesis factor 11 beta \\
\hline ENSG00000103769 & 0.85 & RAB11A & RAB11A, member RAS oncogene family \\
\hline ENSG00000196126 & 0.85 & HLA-DRB2 & $\begin{array}{l}\text { HLA class II histocompatibility antigen, DRB1-1 beta chain } \\
\text { precursor }\end{array}$ \\
\hline ENSG00000108828 & 0.85 & VAT1 & Vesicle amine transport protein 1 homolog (T. californica) \\
\hline ENSG00000180834 & 0.85 & MAP6D1 & MAP6 domain containing 1 \\
\hline ENSG00000138293 & 0.85 & NCOA4 & Nuclear receptor coactivator 4 \\
\hline ENSG00000140598 & 0.85 & EFTUD1 & Elongation factor Tu GTP binding domain containing 1 \\
\hline ENSG00000160703 & 0.85 & NLRX1 & NLR family member $\mathrm{X} 1$ \\
\hline ENSG00000149600 & 0.86 & COMMD7 & COMM domain containing 7 \\
\hline ENSG00000156795 & 0.86 & C8orf32 & NA \\
\hline ENSG00000187838 & 0.86 & PLSCR3 & Phospholipid scramblase 3 \\
\hline ENSG00000116906 & 0.86 & GNPAT & Glyceronephosphate O-acyltransferase \\
\hline ENSG00000153560 & 0.86 & UBP1 & Upstream binding protein 1 (LBP-1a) \\
\hline ENSG00000138279 & 0.86 & ANXA7 & Annexin A7 \\
\hline ENSG00000135446 & 0.86 & CDK4 & Cyclin-dependent kinase 4 \\
\hline ENSG00000100938 & 0.86 & Q86T14_HUMAN & $\begin{array}{l}\text { guanosine monophosphate reductase } 2 \text { (GMPR2), transcript } \\
\text { variant } 2\end{array}$ \\
\hline ENSG00000090432 & 0.86 & C1orf166 & Putative NFkB activating protein \\
\hline ENSG00000101150 & 0.86 & TPD52L2 & Tumor protein D52-like 2 \\
\hline ENSG00000130340 & 0.86 & SNX9 & Sorting nexin 9 \\
\hline ENSG00000137693 & 0.86 & YAP1 & Yes-associated protein $1,65 \mathrm{kDa}$ \\
\hline ENSG00000175193 & 0.86 & PARL & Presenilin associated, rhomboid-like \\
\hline ENSG00000105698 & 0.86 & USF2 & Upstream transcription factor 2, c-fos interacting \\
\hline ENSG00000071626 & 0.86 & DAZAP1 & DAZ associated protein 1 \\
\hline ENSG00000150456 & 0.86 & N6AMT2 & N-6 adenine-specific DNA methyltransferase 2 (putative) \\
\hline ENSG00000170348 & 0.86 & TMED10 & Transmembrane emp24-like trafficking protein 10 (yeast) \\
\hline ENSG00000105856 & 0.86 & HBP1 & HMG-box transcription factor 1 \\
\hline ENSG00000133706 & 0.86 & LARS & Leucyl-tRNA synthetase \\
\hline ENSG00000204218 & 0.86 & RGL2 & Ral guanine nucleotide dissociation stimulator-like 2 \\
\hline ENSG00000100823 & 0.86 & APEX1 & APEX nuclease (multifunctional DNA repair enzyme) 1 \\
\hline ENSG00000147677 & 0.86 & EIF3S3 & Eukaryotic translation initiation factor 3 subunit 3 \\
\hline ENSG00000104969 & 0.86 & SGTA & $\begin{array}{l}\text { Small glutamine-rich tetratricopeptide repeat (TPR)-containing, } \\
\text { alpha }\end{array}$ \\
\hline ENSG00000063978 & 0.86 & RNF4 & Ring finger protein 4 \\
\hline ENSG00000109911 & 0.86 & ELP4 & Elongation protein 4 homolog (S. cerevisiae) \\
\hline ENSG00000114902 & 0.86 & SPCS1 & Signal peptidase complex subunit 1 homolog (S. cerevisiae) \\
\hline ENSG00000148290 & 0.86 & SURF1 & Surfeit 1 \\
\hline ENSG00000162302 & 0.86 & RPS6KA4 & Ribosomal protein S6 kinase, $90 \mathrm{kDa}$, polypeptide 4 \\
\hline
\end{tabular}




\begin{tabular}{|c|c|c|c|}
\hline Ensemble ID & fc & GeneSymbol & Description \\
\hline ENSG00000183283 & 0.86 & DAZAP2 & DAZ associated protein 2 \\
\hline ENSG00000072210 & 0.86 & ALDH3A2 & Aldehyde dehydrogenase 3 family, member A2 \\
\hline ENSG00000126261 & 0.86 & SAE2 & SUMO-activating enzyme subunit 2 \\
\hline ENSG00000103507 & 0.86 & BCKDK & Branched chain ketoacid dehydrogenase kinase \\
\hline ENSG00000115816 & 0.86 & CEBPZ & CCAAT/enhancer binding protein (C/EBP), zeta \\
\hline ENSG00000198858 & 0.86 & C19orf22 & Chromosome 19 open reading frame 22 \\
\hline ENSG00000135486 & 0.86 & HNRPA1 & Heterogeneous nuclear ribonucleoprotein A1 \\
\hline ENSG00000163788 & 0.86 & SNRK & SNF related kinase \\
\hline ENSG00000164182 & 0.86 & NDUFA12L & Mimitin, mitochondrial precursor \\
\hline ENSG00000160767 & 0.86 & C1orf2 & Chromosome 1 open reading frame 2 \\
\hline ENSG00000153574 & 0.86 & RPIA & Ribose 5-phosphate isomerase A \\
\hline ENSG00000056050 & 0.87 & C4orf27 & Chromosome 4 open reading frame 27 \\
\hline ENSG00000121774 & 0.87 & KHDRBS1 & $\begin{array}{l}\mathrm{KH} \text { domain containing, RNA binding, signal transduction } \\
\text { associated } 1\end{array}$ \\
\hline ENSG00000136436 & 0.87 & CALCOCO2 & Calcium binding and coiled-coil domain 2 \\
\hline ENSG00000160058 & 0.87 & BSDC1 & BSD domain containing 1 \\
\hline ENSG00000143149 & 0.87 & ALDH9A1 & Aldehyde dehydrogenase 9 family, member A1 \\
\hline ENSG00000161542 & 0.87 & PRPSAP1 & Phosphoribosyl pyrophosphate synthetase-associated protein 1 \\
\hline ENSG00000145332 & 0.87 & KLHL8 & Kelch-like 8 (Drosophila) \\
\hline ENSG00000013563 & 0.87 & DNASE1L1 & Deoxyribonuclease I-like 1 \\
\hline ENSG00000197879 & 0.87 & MYO1C & Myosin IC \\
\hline ENSG00000125107 & 0.87 & CNOT1 & CCR4-NOT transcription complex, subunit 1 \\
\hline ENSG00000074319 & 0.87 & TSG101 & Tumor susceptibility gene 101 \\
\hline ENSG00000105053 & 0.87 & VRK3 & Vaccinia related kinase 3 \\
\hline ENSG00000074590 & 0.87 & NUAK1 & NUAK family, SNF1-like kinase, 1 \\
\hline ENSG00000112511 & 0.87 & PHF1 & PHD finger protein 1 \\
\hline ENSG00000131269 & 0.87 & $\mathrm{ABCB} 7$ & ATP-binding cassette, sub-family B (MDR/TAP), member 7 \\
\hline ENSG00000204568 & 0.87 & MRPS18B & Mitochondrial ribosomal protein S18B \\
\hline ENSG00000062650 & 0.87 & WAPAL & Wings apart-like protein homolog \\
\hline ENSG00000011009 & 0.87 & LYPLA2 & Lysophospholipase II \\
\hline ENSG00000162032 & 0.87 & SPSB3 & SplA/ryanodine receptor domain and SOCS box containing 3 \\
\hline ENSG00000084234 & 0.87 & APLP2 & Amyloid beta (A4) precursor-like protein 2 \\
\hline ENSG00000102931 & 0.87 & ARL2BP & ADP-ribosylation factor-like 2 binding protein \\
\hline ENSG00000166333 & 0.87 & ILK & Integrin-linked kinase \\
\hline ENSG00000103496 & 0.87 & STX4 & Syntaxin 4 \\
\hline ENSG00000106049 & 0.87 & HIBADH & 3-hydroxyisobutyrate dehydrogenase \\
\hline ENSG00000121067 & 0.87 & SPOP & Speckle-type POZ protein \\
\hline ENSG00000135372 & 0.87 & NAT10 & $\mathrm{N}$-acetyltransferase 10 (GCN5-related) \\
\hline ENSG00000141447 & 0.87 & OSBPL1A & Oxysterol binding protein-like $1 \mathrm{~A}$ \\
\hline ENSG00000139370 & 0.87 & SLC15A4 & Solute carrier family 15 , member 4 \\
\hline ENSG00000182660 & 0.87 & NP_001013698.1 & NA \\
\hline ENSG00000117614 & 0.87 & SYF2 & SYF2 homolog, RNA splicing factor (S. cerevisiae) \\
\hline ENSG00000069329 & 0.87 & VPS35 & Vacuolar protein sorting 35 homolog (S. cerevisiae) \\
\hline ENSG00000165660 & 0.87 & KIAA0157 & NA \\
\hline
\end{tabular}




\begin{tabular}{|c|c|c|c|}
\hline Ensemble ID & fc & GeneSymbol & Description \\
\hline ENSG00000147140 & 0.87 & NONO & Non-POU domain-containing octamer-binding protein \\
\hline ENSG00000161671 & 0.87 & NP_996261.1 & hematopoietic signal peptide-containing isoform 2 \\
\hline ENSG00000120727 & 0.87 & PAIP2 & Polyadenylate-binding protein-interacting protein 2 \\
\hline ENSG00000163161 & 0.87 & ERCC3 & $\begin{array}{l}\text { Excision repair cross-complementing rodent repair deficiency, } \\
\text { complementation group } 3 \text { (xeroderma pigmentosum group B } \\
\text { complementing) }\end{array}$ \\
\hline ENSG00000104679 & 0.87 & $\mathrm{R} 3 \mathrm{HCC} 1$ & $\mathrm{R} 3 \mathrm{H}$ domain and coiled-coil containing 1 \\
\hline ENSG00000102158 & 0.87 & IAG2_HUMAN & Implantation-associated protein precursor \\
\hline ENSG00000167220 & 0.87 & HDHD2 & Haloacid dehalogenase-like hydrolase domain containing 2 \\
\hline ENSG00000110344 & 0.87 & UBE4A & Ubiquitination factor E4A (UFD2 homolog, yeast) \\
\hline ENSG00000136521 & 0.87 & NDUFB5 & $\begin{array}{l}\text { NADH dehydrogenase (ubiquinone) } 1 \text { beta subcomplex, } 5 \text {, } \\
16 \mathrm{kDa}\end{array}$ \\
\hline ENSG00000130429 & 0.87 & ARPC1B & Actin-related protein $2 / 3$ complex subunit 1B \\
\hline ENSG00000116863 & 0.87 & ADPRHL2 & ADP-ribosylhydrolase like 2 \\
\hline ENSG00000135506 & 0.87 & OS9_HUMAN & Protein OS-9 precursor \\
\hline ENSG00000170248 & 0.87 & PDCD6IP & Programmed cell death 6 interacting protein \\
\hline ENSG00000101391 & 0.87 & CDK5RAP1 & CDK5 regulatory subunit associated protein 1 \\
\hline ENSG00000119725 & 0.88 & ZNF410 & Zinc finger protein 410 \\
\hline ENSG00000035141 & 0.88 & NP_116211.1 & NA \\
\hline ENSG00000169180 & 0.88 & XPO6 & Exportin 6 \\
\hline ENSG00000120071 & 0.88 & KIAA1267 & KIAA1267 \\
\hline ENSG00000143549 & 0.88 & TPM3 & Tropomyosin alpha-3 chain \\
\hline ENSG00000144671 & 0.88 & SLC22A14 & Solute carrier family 22 , member 14 \\
\hline ENSG00000116685 & 0.88 & KIAA2013 & KIAA2013 \\
\hline ENSG00000165516 & 0.88 & KLHDC2 & Kelch domain containing 2 \\
\hline ENSG00000114062 & 0.88 & UBE3A & Ubiquitin protein ligase E3A \\
\hline ENSG00000138592 & 0.88 & USP8 & Ubiquitin specific peptidase 8 \\
\hline ENSG00000113312 & 0.88 & TTC1 & Tetratricopeptide repeat domain 1 \\
\hline ENSG00000163539 & 0.88 & CLASP2 & Cytoplasmic linker associated protein 2 \\
\hline ENSG00000158019 & 0.88 & BRE & Brain and reproductive organ-expressed protein \\
\hline ENSG00000100601 & 0.88 & ALKBH1 & AlkB, alkylation repair homolog 1 (E. coli) \\
\hline ENSG00000168894 & 0.88 & NP_057578.1 & NA \\
\hline ENSG00000133193 & 0.88 & FAM104A & Family with sequence similarity 104 , member $\mathrm{A}$ \\
\hline ENSG00000182604 & 0.88 & & NA \\
\hline ENSG00000115073 & 0.88 & ACTR1B & ARP1 actin-related protein 1 homolog B, centractin beta (yeast) \\
\hline ENSG00000132466 & 0.88 & ANKRD17 & Ankyrin repeat domain 17 \\
\hline ENSG00000163069 & 0.88 & SGCB & Sarcoglycan, beta (43kDa dystrophin-associated glycoprotein) \\
\hline ENSG00000134759 & 0.88 & STATIP1 & Stat3-interacting protein \\
\hline ENSG00000186834 & 0.88 & HEXIM1 & Hexamethylene bis-acetamide inducible 1 \\
\hline ENSG00000113558 & 0.88 & SKP1A & S-phase kinase-associated protein $1 \mathrm{~A}$ \\
\hline ENSG00000109445 & 0.88 & ZNF330 & Zinc finger protein 330 \\
\hline ENSG00000138303 & 0.88 & ASCC1 & Activating signal cointegrator 1 complex subunit 1 \\
\hline ENSG00000164366 & 0.88 & CCDC127 & Coiled-coil domain containing 127 \\
\hline ENSG00000175220 & 0.88 & ARHGAP1 & Rho GTPase activating protein 1 \\
\hline ENSG00000116161 & 0.88 & CACYBP & Calcyclin binding protein \\
\hline
\end{tabular}




\begin{tabular}{|c|c|c|c|}
\hline Ensemble ID & fc & GeneSymbol & Description \\
\hline ENSG00000152102 & 0.88 & NP_001009993.2 & p20 \\
\hline ENSG00000101193 & 0.88 & C20orf11 & Chromosome 20 open reading frame 11 \\
\hline ENSG00000153339 & 0.88 & KIAA1012 & KIAA1012 \\
\hline ENSG00000168003 & 0.88 & SLC3A2 & $\begin{array}{l}\text { Solute carrier family } 3 \text { (activators of dibasic and neutral amino } \\
\text { acid transport), member } 2\end{array}$ \\
\hline ENSG00000115317 & 0.88 & HTRA2 & HtrA serine peptidase 2 \\
\hline ENSG00000075856 & 0.89 & SART3 & Squamous cell carcinoma antigen recognized by $\mathrm{T}$ cells 3 \\
\hline ENSG00000174748 & 0.89 & RPL15 & Ribosomal protein L15 \\
\hline ENSG00000110442 & 0.89 & COMMD9 & COMM domain containing 9 \\
\hline ENSG00000095209 & 0.89 & TMEM38B & Transmembrane protein 38B \\
\hline ENSG00000078369 & 0.89 & GNB1 & $\begin{array}{l}\text { Guanine nucleotide binding protein (G protein), beta } \\
\text { polypeptide } 1\end{array}$ \\
\hline ENSG00000003393 & 0.89 & ALS2 & Amyotrophic lateral sclerosis 2 (juvenile) \\
\hline ENSG00000175756 & 0.89 & AURKAIP1 & Aurora kinase $\mathrm{A}$ interacting protein 1 \\
\hline ENSG00000198176 & 0.89 & TFDP1 & Transcription factor Dp-1 \\
\hline ENSG00000125944 & 0.89 & HNRPR & Heterogeneous nuclear ribonucleoprotein $\mathrm{R}$ \\
\hline ENSG00000171604 & 0.89 & CXXC5 & CXXC finger 5 \\
\hline ENSG00000107021 & 0.89 & TBC1D13 & TBC1 domain family, member 13 \\
\hline ENSG00000077097 & 0.89 & TOP2B & Topoisomerase (DNA) II beta $180 \mathrm{kDa}$ \\
\hline ENSG00000173011 & 0.89 & Q86TJ2_HUMAN & NA \\
\hline ENSG00000156990 & 0.89 & RPUSD3 & RNA pseudouridylate synthase domain containing 3 \\
\hline ENSG00000177885 & 0.89 & GRB2 & Growth factor receptor-bound protein 2 \\
\hline ENSG00000158417 & 0.89 & EIF5B & Eukaryotic translation initiation factor $5 \mathrm{~B}$ \\
\hline ENSG00000165943 & 0.89 & MOAP1 & Modulator of apoptosis 1 \\
\hline ENSG00000173812 & 0.89 & EIF1 & Eukaryotic translation initiation factor 1 \\
\hline ENSG00000204439 & 0.89 & C6orf47 & Chromosome 6 open reading frame 47 \\
\hline ENSG00000117408 & 0.89 & IPO13 & Importin 13 \\
\hline ENSG00000084093 & 0.89 & REST & RE1-silencing transcription factor \\
\hline ENSG00000108840 & 0.89 & HDAC5 & Histone deacetylase 5 \\
\hline ENSG00000147364 & 0.89 & FBXO25 & F-box protein 25 \\
\hline ENSG00000068697 & 0.89 & LAPTM4A & Lysosomal protein transmembrane 4 alpha \\
\hline ENSG00000153250 & 0.89 & RBMS1 & RNA binding motif, single stranded interacting protein 1 \\
\hline ENSG00000119638 & 0.89 & NEK9 & NIMA (never in mitosis gene a)- related kinase 9 \\
\hline ENSG00000104824 & 0.89 & HNRPL & Heterogeneous nuclear ribonucleoprotein $\mathrm{L}$ \\
\hline ENSG00000078140 & 0.89 & HIP2 & $\begin{array}{l}\text { Ubiquitin-conjugating enzyme E2-25 kDa (EC 6.3.2.19) } \\
\text { (Ubiquitin- protein ligase) (Ubiquitin carrier protein) (E2(25K)) } \\
\text { (Huntingtin- interacting protein 2) (HIP-2). } \\
\text { [Source:Uniprot/SWISSPROT;Acc:P61086] }\end{array}$ \\
\hline ENSG00000163320 & 0.89 & & CGG triplet repeat binding protein 1 \\
\hline ENSG00000180632 & 0.89 & & NA \\
\hline ENSG00000154582 & 0.89 & TCEB1 & $\begin{array}{l}\text { Transcription elongation factor B (SIII), polypeptide } 1 \text { ( } 15 \mathrm{kDa} \text {, } \\
\text { elongin C) }\end{array}$ \\
\hline ENSG00000114650 & 0.89 & SCAP & SREBF chaperone \\
\hline ENSG00000163820 & 0.89 & FYCO1 & FYVE and coiled-coil domain containing 1 \\
\hline ENSG00000148925 & 0.90 & BTBD10 & BTB (POZ) domain containing 10 \\
\hline ENSG00000111642 & 0.90 & CHD4 & Chromodomain helicase DNA binding protein 4 \\
\hline ENSG00000102921 & 0.90 & N4BP1_HUMAN & NEDD4-binding protein 1 \\
\hline
\end{tabular}




\begin{tabular}{|c|c|c|c|}
\hline Ensemble ID & fc & GeneSymbol & Description \\
\hline ENSG00000112335 & 0.90 & SNX3 & Sorting nexin 3 \\
\hline ENSG00000111641 & 0.90 & NOL1 & Proliferating-cell nucleolar antigen p120 \\
\hline ENSG00000114388 & 0.90 & TUSC4 & Tumor suppressor candidate 4 \\
\hline ENSG00000115307 & 0.90 & AUP1 & Ancient ubiquitous protein 1 \\
\hline ENSG00000114054 & 0.90 & PCCB & Propionyl Coenzyme A carboxylase, beta polypeptide \\
\hline ENSG00000142230 & 0.90 & SAE1 & SUMO1 activating enzyme subunit 1 \\
\hline ENSG00000168288 & 0.90 & C2orf25 & NA \\
\hline ENSG00000161835 & 0.90 & GRASP & $\begin{array}{l}\text { GRP1 (general receptor for phosphoinositides 1)-associated } \\
\text { scaffold protein }\end{array}$ \\
\hline ENSG00000162734 & 0.90 & PEA15 & Phosphoprotein enriched in astrocytes 15 \\
\hline ENSG00000162961 & 0.90 & DPY30_HUMAN & Dpy-30-like protein \\
\hline ENSG00000108963 & 0.90 & DPH1 & DPH1 homolog (S. cerevisiae) \\
\hline ENSG00000107897 & 0.90 & ACBD5 & Acyl-Coenzyme A binding domain containing 5 \\
\hline ENSG00000104067 & 0.90 & TJP1 & Tight junction protein 1 (zona occludens 1 ) \\
\hline ENSG00000083454 & 0.90 & TAX1BP3 & Tax1 (human T-cell leukemia virus type I) binding protein 3 \\
\hline ENSG00000071889 & 0.90 & FAM3A & Family with sequence similarity 3 , member $A$ \\
\hline ENSG00000078070 & 0.90 & MCCC1 & Methylcrotonoyl-Coenzyme A carboxylase 1 (alpha) \\
\hline ENSG00000105648 & 0.90 & IFI30 & Interferon, gamma-inducible protein 30 \\
\hline ENSG00000144647 & 0.90 & C3orf39 & Chromosome 3 open reading frame 39 \\
\hline ENSG00000122783 & 0.91 & C7orf49 & Chromosome 7 open reading frame 49 \\
\hline ENSG00000146701 & 0.91 & $\mathrm{MDH} 2$ & Malate dehydrogenase 2, NAD (mitochondrial) \\
\hline ENSG00000123130 & 0.91 & ACOT9 & Acyl-CoA thioesterase 9 \\
\hline ENSG00000169217 & 0.91 & CD2BP2 & CD2 (cytoplasmic tail) binding protein 2 \\
\hline ENSG00000142168 & 0.91 & SOD1 & Superoxide dismutase 1 , soluble \\
\hline ENSG00000145362 & 0.91 & ANK2 & Ankyrin 2, neuronal \\
\hline ENSG00000085871 & 0.91 & MGST2 & Microsomal glutathione S-transferase 2 \\
\hline ENSG00000131966 & 0.91 & ACTR10 & Actin-related protein 10 homolog (S. cerevisiae) \\
\hline ENSG00000119844 & 1.13 & AFTPH & Aftiphilin \\
\hline ENSG00000082996 & 1.13 & RNF13 & Ring finger protein 13 \\
\hline ENSG00000144677 & 1.13 & CTDSPL & $\begin{array}{l}\text { CTD (carboxy-terminal domain, RNA polymerase II, polypeptide } \\
\text { A) small phosphatase-like }\end{array}$ \\
\hline ENSG00000166479 & 1.13 & TXNDC10 & Protein disulfide-isomerase \\
\hline ENSG00000138385 & 1.14 & SSB & Sjogren syndrome antigen B (autoantigen La) \\
\hline ENSG00000105583 & 1.14 & C19orf56 & Chromosome 19 open reading frame 56 \\
\hline ENSG00000054267 & 1.14 & ARID4B & AT rich interactive domain 4B (RBP1-like) \\
\hline ENSG00000162694 & 1.14 & EXTL2 & Exostoses (multiple)-like 2 \\
\hline ENSG00000189227 & 1.14 & LOC145853 & NA \\
\hline ENSG00000153037 & 1.14 & SRP19 & Signal recognition particle $19 \mathrm{kDa}$ \\
\hline ENSG00000064726 & 1.15 & BTBD1 & BTB (POZ) domain containing 1 \\
\hline ENSG00000169926 & 1.15 & KLF13 & Kruppel-like factor 13 \\
\hline ENSG00000090266 & 1.15 & NDUFB2 & $\begin{array}{l}\text { NADH dehydrogenase (ubiquinone) } 1 \text { beta subcomplex, } 2 \text {, } \\
8 \mathrm{kDa}\end{array}$ \\
\hline ENSG00000165874 & 1.15 & FAM35A & Family with sequence similarity 35, member $A$ \\
\hline ENSG00000135698 & 1.16 & MPHOSPH6 & M-phase phosphoprotein 6 \\
\hline ENSG00000113387 & 1.16 & SUB1 & SUB1 homolog (S. cerevisiae) \\
\hline
\end{tabular}




\begin{tabular}{|c|c|c|c|}
\hline Ensemble ID & fc & GeneSymbol & Description \\
\hline ENSG00000183513 & 1.16 & NP_001008216.1 & NA \\
\hline ENSG00000166710 & 1.16 & B2M & Beta-2-microglobulin \\
\hline ENSG00000104231 & 1.16 & ZFAND1 & Zinc finger, AN1-type domain 1 \\
\hline ENSG00000102897 & 1.16 & LYRM1 & LYR motif containing 1 \\
\hline ENSG00000079134 & 1.16 & THOC1 & THO complex 1 \\
\hline ENSG00000144785 & 1.16 & TMEM4 & MIR-interacting saposin-like protein precursor \\
\hline ENSG00000205560 & 1.16 & $\mathrm{CHKB}$ & Choline kinase beta \\
\hline ENSG00000127184 & 1.16 & COX7C & Cytochrome c oxidase subunit VIlc \\
\hline ENSG00000182670 & 1.16 & TTC3 & Tetratricopeptide repeat domain 3 \\
\hline ENSG00000138035 & 1.16 & PNPT1 & Polyribonucleotide nucleotidyltransferase 1 \\
\hline ENSG00000115514 & 1.17 & TXNDC9 & Thioredoxin domain containing 9 \\
\hline ENSG00000119013 & 1.17 & NDUFB3 & $\begin{array}{l}\text { NADH dehydrogenase (ubiquinone) } 1 \text { beta subcomplex, } 3 \text {, } \\
12 \mathrm{kDa}\end{array}$ \\
\hline ENSG00000166200 & 1.17 & COPS2 & $\begin{array}{l}\text { COP9 constitutive photomorphogenic homolog subunit } 2 \\
\text { (Arabidopsis) }\end{array}$ \\
\hline ENSG00000164828 & 1.17 & UNC84A & Unc-84 homolog A (C. elegans) \\
\hline ENSG00000116747 & 1.18 & TROVE2 & TROVE domain family, member 2 \\
\hline ENSG00000161547 & 1.18 & SFRS2 & Splicing factor, arginine/serine-rich 2 \\
\hline ENSG00000124593 & 1.18 & C6orf49 & LIM domain-containing protein \\
\hline ENSG00000127540 & 1.18 & UQCR & Ubiquinol-cytochrome $\mathrm{c}$ reductase, $6.4 \mathrm{kDa}$ subunit \\
\hline ENSG00000164898 & 1.18 & NP_932068.1 & NA \\
\hline ENSG00000120333 & 1.18 & MRPS14 & Mitochondrial ribosomal protein S14 \\
\hline ENSG00000118939 & 1.18 & UCHL3 & Ubiquitin carboxyl-terminal esterase L3 (ubiquitin thiolesterase) \\
\hline ENSG00000143198 & 1.18 & MGST3 & Microsomal glutathione S-transferase 3 \\
\hline ENSG00000116560 & 1.18 & SFPQ & $\begin{array}{l}\text { Splicing factor proline/glutamine-rich (polypyrimidine tract } \\
\text { binding protein associated) }\end{array}$ \\
\hline ENSG00000127720 & 1.19 & C12orf26 & Chromosome 12 open reading frame 26 \\
\hline ENSG00000070501 & 1.19 & POLB & Polymerase (DNA directed), beta \\
\hline ENSG00000100387 & 1.19 & RBX1 & Ring-box 1 \\
\hline ENSG00000115414 & 1.19 & FN1 & Fibronectin 1 \\
\hline ENSG00000134253 & 1.19 & TRIM45 & Tripartite motif-containing 45 \\
\hline ENSG00000147027 & 1.19 & TMEM47 & Transmembrane protein 47 \\
\hline ENSG00000100211 & 1.19 & PGEA1 & PKD2 interactor, Golgi and endoplasmic reticulum associated 1 \\
\hline ENSG00000066557 & 1.19 & LRRC40 & Leucine rich repeat containing 40 \\
\hline ENSG00000132274 & 1.19 & TRIM22 & Tripartite motif-containing protein 22 \\
\hline ENSG00000156467 & 1.20 & UQCRB & Ubiquinol-cytochrome $\mathrm{c}$ reductase binding protein \\
\hline ENSG00000138801 & 1.20 & PAPSS1 & 3'-phosphoadenosine 5'-phosphosulfate synthase 1 \\
\hline ENSG00000167280 & 1.20 & NP_001036038.1 & endo-beta- $\mathrm{N}$-acetylglucosaminidase \\
\hline ENSG00000151500 & 1.20 & THYN1 & Thymocyte nuclear protein 1 \\
\hline ENSG00000203743 & 1.20 & Q5J7V3_HUMAN & Migration-inducing gene 16 protein \\
\hline ENSG00000076513 & 1.20 & ANKRD13A & Ankyrin repeat domain $13 \mathrm{~A}$ \\
\hline ENSG00000181038 & 1.20 & Q8N712_HUMAN & NA \\
\hline ENSG00000204370 & 1.20 & SDHD & $\begin{array}{l}\text { Succinate dehydrogenase complex, subunit } D \text {, integral } \\
\text { membrane protein }\end{array}$ \\
\hline ENSG00000115350 & 1.21 & POLE4 & Polymerase (DNA-directed), epsilon 4 ( $p 12$ subunit) \\
\hline ENSG00000163797 & 1.21 & MRPL33 & Mitochondrial ribosomal protein L33 \\
\hline
\end{tabular}




\begin{tabular}{|c|c|c|c|}
\hline Ensemble ID & fc & GeneSymbol & Description \\
\hline ENSG00000162407 & 1.21 & PPAP2B & Lipid phosphate phosphohydrolase 3 \\
\hline ENSG00000006625 & 1.21 & C7orf24 & NA \\
\hline ENSG00000166147 & 1.21 & FBN1 & Fibrillin 1 \\
\hline ENSG00000103121 & 1.21 & C16orf61 & Chromosome 16 open reading frame 61 \\
\hline ENSG00000182117 & 1.21 & NOLA3 & $\mathrm{H} / \mathrm{ACA}$ ribonucleoprotein complex subunit 3 \\
\hline ENSG00000171862 & 1.21 & PTENP1 & Phosphatase and tensin homolog pseudogene 1 \\
\hline ENSG00000165672 & 1.22 & PRDX3 & Thioredoxin-dependent peroxide reductase \\
\hline ENSG00000100697 & 1.22 & DICER1 & Dicer 1 , ribonuclease type III \\
\hline ENSG00000122033 & 1.23 & MTIF3 & Mitochondrial translational initiation factor 3 \\
\hline ENSG00000116717 & 1.23 & GADD45A & Growth arrest and DNA-damage-inducible, alpha \\
\hline ENSG00000182600 & 1.23 & NP_996778.1 & NA \\
\hline ENSG00000165264 & 1.23 & NDUFB6 & $\begin{array}{l}\text { NADH dehydrogenase (ubiquinone) } 1 \text { beta subcomplex, } 6 \text {, } \\
17 \mathrm{kDa}\end{array}$ \\
\hline ENSG00000111237 & 1.23 & VPS29 & Vacuolar protein sorting 29 homolog (S. cerevisiae) \\
\hline ENSG00000136161 & 1.23 & RCBTB2 & $\begin{array}{l}\text { Regulator of chromosome condensation (RCC1) and BTB } \\
\text { (POZ) domain containing protein } 2\end{array}$ \\
\hline ENSG00000173027 & 1.23 & WBP1 & WW domain binding protein 1 \\
\hline ENSG00000023041 & 1.23 & ZDHHC6 & Zinc finger, DHHC-type containing 6 \\
\hline ENSG00000125676 & 1.24 & THOC2 & THO complex 2 \\
\hline ENSG00000196549 & 1.24 & MME & Membrane metallo-endopeptidase \\
\hline ENSG00000196233 & 1.24 & LCOR & Ligand dependent nuclear receptor corepressor \\
\hline ENSG00000160808 & 1.24 & MYL3 & Myosin, light chain 3, alkali; ventricular, skeletal, slow \\
\hline ENSG00000005075 & 1.24 & POLR2J & Polymerase (RNA) II (DNA directed) polypeptide J, 13.3kDa \\
\hline ENSG00000157510 & 1.24 & NP_689619.1 & NA \\
\hline ENSG00000144182 & 1.24 & LIPT1 & $\begin{array}{l}\text { Lipoyltransferase 1, mitochondrial precursor (EC 6.-.-.-) } \\
\text { (Lipoate- protein ligase) (Lipoate biosynthesis protein) (Lipoyl } \\
\text { ligase). [Source:Uniprot/SWISSPROT;Acc:Q9Y234] }\end{array}$ \\
\hline ENSG00000183617 & 1.24 & MRPL54 & Mitochondrial ribosomal protein L54 \\
\hline ENSG00000197335 & 1.24 & & NA \\
\hline ENSG00000196683 & 1.24 & TOMM7 & $\begin{array}{l}\text { Translocase of outer mitochondrial membrane } 7 \text { homolog } \\
\text { (yeast) }\end{array}$ \\
\hline ENSG00000027697 & 1.25 & IFNGR1 & Interferon gamma receptor 1 \\
\hline ENSG00000172171 & 1.25 & C17orf42 & Chromosome 17 open reading frame 42 \\
\hline ENSG00000137500 & 1.25 & CCDC90B & Coiled-coil domain containing 90B \\
\hline ENSG00000071794 & 1.25 & HLTF & Helicase-like transcription factor \\
\hline ENSG00000136147 & 1.25 & PHF11 & PHD finger protein 11 \\
\hline ENSG00000139617 & 1.25 & NP_055702.1 & phosphonoformate immuno-associated protein 5 \\
\hline ENSG00000130508 & 1.25 & PXDN & Peroxidasin homolog (Drosophila) \\
\hline ENSG00000163520 & 1.25 & FBLN2 & Fibulin 2 \\
\hline ENSG00000106591 & 1.25 & MRPL32 & Mitochondrial ribosomal protein L32 \\
\hline ENSG00000169567 & 1.26 & HINT1 & Histidine triad nucleotide binding protein 1 \\
\hline ENSG00000114209 & 1.26 & PDCD10 & Programmed cell death 10 \\
\hline ENSG00000059588 & 1.26 & TARBP1 & TAR (HIV-1) RNA binding protein 1 \\
\hline ENSG00000149273 & 1.26 & RPS3 & Ribosomal protein S3 \\
\hline ENSG00000143933 & 1.27 & CALM2 & Calmodulin 2 (phosphorylase kinase, delta) \\
\hline ENSG00000173915 & 1.27 & USMG5 & Up-regulated during skeletal muscle growth 5 homolog (mouse) \\
\hline
\end{tabular}




\begin{tabular}{|c|c|c|c|}
\hline Ensemble ID & fc & GeneSymbol & Description \\
\hline ENSG00000112893 & 1.27 & MAN2A1 & Mannosidase, alpha, class 2A, member 1 \\
\hline ENSG00000204256 & 1.27 & BRD2 & Bromodomain containing 2 \\
\hline ENSG00000118292 & 1.28 & C1orf54 & Chromosome 1 open reading frame 54 \\
\hline ENSG00000173221 & 1.28 & GLRX & Glutaredoxin (thioltransferase) \\
\hline ENSG00000134490 & 1.28 & C18orf45 & Chromosome 18 open reading frame 45 \\
\hline ENSG00000143442 & 1.29 & POGZ & Pogo transposable element with ZNF domain \\
\hline ENSG00000205707 & 1.29 & LYRM5 & LYR motif containing 5 \\
\hline ENSG00000108381 & 1.29 & ASPA & Aspartoacylase (Canavan disease) \\
\hline ENSG00000169976 & 1.29 & SF3B5 & Splicing factor $3 \mathrm{~b}$, subunit $5,10 \mathrm{kDa}$ \\
\hline ENSG00000139679 & 1.30 & P2RY5 & Purinergic receptor P2Y, G-protein coupled, 5 \\
\hline ENSG00000099964 & 1.30 & MIF & $\begin{array}{l}\text { Macrophage migration inhibitory factor (glycosylation-inhibiting } \\
\text { factor) }\end{array}$ \\
\hline ENSG00000068615 & 1.31 & REEP1 & Receptor accessory protein 1 \\
\hline ENSG00000110077 & 1.31 & MS4A6A & Membrane-spanning 4-domains, subfamily $A$, member $6 \mathrm{~A}$ \\
\hline ENSG00000116754 & 1.31 & SFRS11 & Splicing factor, arginine/serine-rich 11 \\
\hline ENSG00000189043 & 1.31 & NDUFA4 & $\begin{array}{l}\text { NADH dehydrogenase (ubiquinone) } 1 \text { alpha subcomplex, } 4 \text {, } \\
9 \mathrm{kDa}\end{array}$ \\
\hline ENSG00000137210 & 1.32 & TMEM14B & Transmembrane protein 14B \\
\hline ENSG00000109270 & 1.32 & MAP2K1IP1 & Mitogen-activated protein kinase kinase 1-interacting protein 1 \\
\hline ENSG00000160916 & 1.32 & ATP5J2 & ATP synthase $f$ chain, mitochondrial \\
\hline ENSG00000206528 & 1.32 & Q6ZSM0_HUMAN & NA \\
\hline ENSG00000109238 & 1.32 & COX7A3 & Cytochrome c oxidase subunit VIla 3 \\
\hline ENSG00000177600 & 1.32 & RPLP2 & Ribosomal protein, large, P2 \\
\hline ENSG00000124193 & 1.33 & SFRS6 & Splicing factor, arginine/serine-rich 6 \\
\hline ENSG00000107020 & 1.33 & C9orf46 & Chromosome 9 open reading frame 46 \\
\hline ENSG00000115963 & 1.33 & RND3 & Rho family GTPase 3 \\
\hline ENSG00000156411 & 1.33 & C14orf2 & Chromosome 14 open reading frame 2 \\
\hline ENSG00000034510 & 1.34 & TMSB10 & Thymosin beta 10 \\
\hline ENSG00000184575 & 1.34 & XPOT & Exportin, tRNA (nuclear export receptor for tRNAs) \\
\hline ENSG00000118418 & 1.34 & HMGN3 & High mobility group nucleosomal binding domain 3 \\
\hline ENSG00000122643 & 1.34 & NT5C3 & 5'-nucleotidase, cytosolic III \\
\hline ENSG00000005810 & 1.34 & MYCBP2 & MYC binding protein 2 \\
\hline ENSG00000155367 & 1.34 & PPM1J & Protein phosphatase $1 \mathrm{~J}$ (PP2C domain containing) \\
\hline ENSG00000088448 & 1.34 & ANKRD10 & Ankyrin repeat domain 10 \\
\hline ENSG00000175197 & 1.34 & DDIT3 & DNA damage-inducible transcript 3 \\
\hline ENSG00000188447 & 1.35 & LOC390604 & NA \\
\hline ENSG00000010322 & 1.35 & $\mathrm{NISCH}$ & Nischarin \\
\hline ENSG00000104408 & 1.35 & EIF3S6 & Eukaryotic translation initiation factor 3 subunit 6 \\
\hline ENSG00000039319 & 1.35 & ZFYVE16 & Zinc finger, FYVE domain containing 16 \\
\hline ENSG00000204820 & 1.35 & Q96NT9_HUMAN & GR AF-1 specific protein phosphatase \\
\hline ENSG00000183963 & 1.35 & SMTN & Smoothelin \\
\hline ENSG00000172172 & 1.35 & MRPL13 & Mitochondrial ribosomal protein L13 \\
\hline ENSG00000178449 & 1.35 & C12orf62 & Chromosome 12 open reading frame 62 \\
\hline ENSG00000140450 & 1.35 & ARRDC4 & Arrestin domain containing 4 \\
\hline
\end{tabular}




\begin{tabular}{|c|c|c|c|}
\hline Ensemble ID & fc & GeneSymbol & Description \\
\hline ENSG00000111341 & 1.35 & MGP & Matrix Gla protein \\
\hline ENSG00000183648 & 1.35 & NDUFB1 & $\begin{array}{l}\text { NADH dehydrogenase (ubiquinone) } 1 \text { beta subcomplex, } 1 \text {, } \\
7 \mathrm{kDa}\end{array}$ \\
\hline ENSG00000102760 & 1.35 & C13orf15 & Chromosome 13 open reading frame 15 \\
\hline ENSG00000198523 & 1.36 & PLN & Phospholamban \\
\hline ENSG00000165792 & 1.36 & METT11D1 & Methyltransferase 11 domain containing 1 \\
\hline ENSG00000198235 & 1.36 & & similar to $40 \mathrm{~S}$ ribosomal protein $\mathrm{S} 17$ \\
\hline ENSG00000136783 & 1.36 & NIPSNAP3A & Nipsnap homolog 3A (C. elegans) \\
\hline ENSG00000129152 & 1.37 & MYOD1 & Myogenic differentiation 1 \\
\hline ENSG00000144476 & 1.37 & CXCR7 & Chemokine (C-X-C motif) receptor 7 \\
\hline ENSG00000161281 & 1.37 & COX7A1 & Cytochrome c oxidase subunit VIla polypeptide 1 (muscle) \\
\hline ENSG00000132485 & 1.37 & ZRANB2 & Zinc finger, RAN-binding domain containing 2 \\
\hline ENSG00000132541 & 1.38 & HRSP12 & Heat-responsive protein 12 \\
\hline ENSG00000121310 & 1.38 & ECHDC2 & Enoyl Coenzyme A hydratase domain containing 2 \\
\hline ENSG00000175768 & 1.38 & C9orf105 & NA \\
\hline ENSG00000178950 & 1.38 & GAK & Cyclin $\mathrm{G}$ associated kinase \\
\hline ENSG00000154553 & 1.38 & PDLIM3 & PDZ and LIM domain 3 \\
\hline ENSG00000116791 & 1.39 & CRYZ & Crystallin, zeta (quinone reductase) \\
\hline ENSG00000112146 & 1.39 & FBXO9 & F-box protein 9 \\
\hline ENSG00000113240 & 1.40 & CLK4 & CDC-like kinase 4 \\
\hline ENSG00000156931 & 1.40 & VPS8 & Vacuolar protein sorting 8 homolog (S. cerevisiae) \\
\hline ENSG00000164919 & 1.40 & $\mathrm{COX} 6 \mathrm{C}$ & Cytochrome c oxidase subunit VIc \\
\hline ENSG00000092841 & 1.40 & MYL6 & Myosin, light chain 6, alkali, smooth muscle and non-muscle \\
\hline ENSG00000163170 & 1.40 & BOLA3 & BolA homolog 3 (E. coli) \\
\hline ENSG00000124370 & 1.40 & MCEE & Methylmalonyl CoA epimerase \\
\hline ENSG00000166136 & 1.42 & NDUFB8 & $\begin{array}{l}\text { NADH dehydrogenase (ubiquinone) } 1 \text { beta subcomplex, } 8 \text {, } \\
19 \mathrm{kDa}\end{array}$ \\
\hline ENSG00000197969 & 1.42 & VPS13A & Vacuolar protein sorting 13 homolog A (S. cerevisiae) \\
\hline ENSG00000185222 & 1.42 & WBP5 & WW domain binding protein 5 \\
\hline ENSG00000100320 & 1.42 & RBM9 & RNA binding motif protein 9 \\
\hline ENSG00000204262 & 1.43 & COL5A2 & Collagen, type $\mathrm{V}$, alpha 2 \\
\hline ENSG00000011465 & 1.43 & DCN & Decorin \\
\hline ENSG00000182457 & 1.43 & Q9NWT5_HUMAN & taurine upregulated gene 1 \\
\hline ENSG00000122566 & 1.44 & HNRPA2B1 & Heterogeneous nuclear ribonucleoproteins A2/B1 \\
\hline ENSG00000176261 & 1.45 & ZBTB8OS & Zinc finger and BTB domain containing 8 opposite strand \\
\hline ENSG00000127990 & 1.45 & SGCE & Sarcoglycan, epsilon \\
\hline ENSG00000166012 & 1.46 & SNORA32 & Small nucleolar RNA, H/ACA box 32 \\
\hline ENSG00000167468 & 1.46 & GPX4 & Glutathione peroxidase 4 (phospholipid hydroperoxidase) \\
\hline ENSG00000127914 & 1.46 & AKAP9 & A kinase (PRKA) anchor protein (yotiao) 9 \\
\hline ENSG00000137288 & 1.46 & C6orf125 & Chromosome 6 open reading frame 125 \\
\hline ENSG00000116489 & 1.47 & CAPZA1 & Capping protein (actin filament) muscle Z-line, alpha 1 \\
\hline ENSG00000144034 & 1.48 & TPRKB & TP53RK binding protein \\
\hline ENSG00000179085 & 1.48 & DPM3 & Dolichyl-phosphate mannosyltransferase polypeptide 3 \\
\hline ENSG00000186566 & 1.48 & GPATCH8 & G patch domain containing 8 \\
\hline
\end{tabular}




\begin{tabular}{|c|c|c|c|}
\hline Ensemble ID & fc & GeneSymbol & Description \\
\hline ENSG00000173960 & 1.49 & UBXD4 & UBX domain-containing protein 4 \\
\hline ENSG00000125730 & 1.49 & $\mathrm{C} 3$ & Complement component 3 \\
\hline ENSG00000104213 & 1.49 & PDGFRL & Platelet-derived growth factor receptor-like \\
\hline ENSG00000148700 & 1.49 & ADD3 & Adducin 3 (gamma) \\
\hline ENSG00000176718 & 1.50 & C5orf26 & Chromosome 5 open reading frame 26 \\
\hline ENSG00000134853 & 1.51 & PDGFRA & Platelet-derived growth factor receptor, alpha polypeptide \\
\hline ENSG00000185847 & 1.51 & Q8IVN4_HUMAN & NA \\
\hline ENSG00000198695 & 1.52 & MT-ND6 & $\mathrm{NADH}$-ubiquinone oxidoreductase chain 6 \\
\hline ENSG00000168002 & 1.52 & POLR2G & Polymerase (RNA) II (DNA directed) polypeptide G \\
\hline ENSG00000131016 & 1.53 & AKAP12 & A kinase (PRKA) anchor protein 12 \\
\hline ENSG00000119927 & 1.53 & GPAM & Glycerol-3-phosphate acyltransferase, mitochondrial \\
\hline ENSG00000115934 & 1.54 & PPIL3 & Peptidylprolyl isomerase (cyclophilin)-like 3 \\
\hline ENSG00000100650 & 1.54 & SFRS5 & Splicing factor, arginine/serine-rich 5 \\
\hline ENSG00000100941 & 1.54 & PNN & Pinin, desmosome associated protein \\
\hline ENSG00000114770 & 1.55 & $\mathrm{ABCC} 5$ & ATP-binding cassette, sub-family C (CFTR/MRP), member 5 \\
\hline ENSG00000101938 & 1.55 & CHRDL1 & Chordin-like 1 \\
\hline ENSG00000139329 & 1.55 & LUM & Lumican \\
\hline ENSG00000147421 & 1.56 & HMBOX1 & Homeobox containing 1 \\
\hline ENSG00000169019 & 1.56 & COMMD8 & COMM domain containing 8 \\
\hline ENSG00000186166 & 1.56 & CCDC84 & Coiled-coil domain containing 84 \\
\hline ENSG00000166681 & 1.56 & NGFRAP1 & Nerve growth factor receptor-associated protein 1 \\
\hline ENSG00000171204 & 1.56 & TMEM126B & Transmembrane protein 126B \\
\hline ENSG00000125995 & 1.57 & C20orf52 & NA \\
\hline ENSG00000003756 & 1.59 & RBM5 & RNA-binding protein 5 \\
\hline ENSG00000141338 & 1.60 & ABCA8 & ATP-binding cassette, sub-family A (ABC1), member 8 \\
\hline ENSG00000115592 & 1.60 & PRKAG3 & Protein kinase, AMP-activated, gamma 3 non-catalytic subunit \\
\hline ENSG00000006652 & 1.62 & IFRD1 & Interferon-related developmental regulator 1 \\
\hline ENSG00000119705 & 1.62 & C14orf156 & Chromosome 14 open reading frame 156 \\
\hline ENSG00000135046 & 1.63 & ANXA1 & Annexin A1 \\
\hline ENSG00000106484 & 1.64 & MEST & Mesoderm specific transcript homolog (mouse) \\
\hline ENSG00000168542 & 1.64 & COL3A1 & Collagen, type III, alpha 1 \\
\hline ENSG00000204460 & 1.65 & Q499Y1_HUMAN & NA \\
\hline ENSG00000089335 & 1.65 & ZNF302 & Zinc finger protein 302 \\
\hline ENSG00000009413 & 1.66 & REV3L & REV3-like, catalytic subunit of DNA polymerase zeta (yeast) \\
\hline ENSG00000204291 & 1.66 & COL15A1 & Collagen, type XV, alpha 1 \\
\hline ENSG00000186594 & 1.67 & NP_001001870.1 & NA \\
\hline ENSG00000154188 & 1.67 & ANGPT1 & Angiopoietin 1 \\
\hline ENSG00000132680 & 1.68 & KIAA0907 & KIAA0907 \\
\hline ENSG00000207786 & 1.70 & hsa-mir-133a-1 & NA \\
\hline ENSG00000181722 & 1.75 & ZBTB20 & Zinc finger and BTB domain containing 20 \\
\hline ENSG00000123689 & 1.76 & GoS2 & Putative lymphocyte G0/G1 switch protein 2 \\
\hline ENSG00000164692 & 1.77 & COL1A2 & Collagen, type I, alpha 2 \\
\hline ENSG00000108773 & 1.79 & GCN5L2 & General control of amino acid synthesis protein 5 -like 2 \\
\hline
\end{tabular}




\begin{tabular}{|c|c|c|c|}
\hline Ensemble ID & fc & GeneSymbol & Description \\
\hline ENSG00000116148 & 1.81 & CCNL2 & Cyclin-L2 \\
\hline ENSG00000123636 & 1.89 & BAZ2B & Bromodomain adjacent to zinc finger domain, $2 \mathrm{~B}$ \\
\hline ENSG00000121350 & 1.89 & NP_079130.2 & NA \\
\hline ENSG00000188536 & 1.94 & HBA1 & Hemoglobin, alpha 1 \\
\hline ENSG00000132424 & 2.00 & C6orf111 & Splicing factor, arginine/serine-rich 130 \\
\hline ENSG00000175606 & 2.01 & TMEM70 & Transmembrane protein 70 \\
\hline ENSG00000198722 & 2.01 & UNC13B & Unc-13 homolog B (C. elegans) \\
\hline ENSG00000108654 & 2.04 & DDX5 & DEAD (Asp-Glu-Ala-Asp) box polypeptide 5 \\
\hline ENSG00000114120 & 2.08 & SLC25A36 & Solute carrier family 25, member 36 \\
\hline ENSG00000109920 & 2.09 & FNBP4 & Formin binding protein 4 \\
\hline ENSG00000129204 & 2.16 & USP6 & Ubiquitin specific peptidase 6 (Tre-2 oncogene) \\
\hline ENSG00000013441 & 2.16 & CLK1 & CDC-like kinase 1 \\
\hline ENSG00000189058 & 2.29 & APOD & Apolipoprotein D \\
\hline ENSG00000108848 & 2.48 & CROP_HUMAN & Cisplatin resistance-associated overexpressed protein \\
\hline ENSG00000166348 & 2.57 & USP54 & Ubiquitin specific peptidase 54 \\
\hline ENSG00000109061 & 2.60 & MYH1 & Myosin, heavy chain 1 , skeletal muscle, adult \\
\hline ENSG00000184027 & 2.75 & MALAT_HUMAN & Metastasis-associated lung adenocarcinoma transcript 1 \\
\hline ENSG00000109046 & 2.93 & WSB1 & WD repeat and SOCS box-containing 1 \\
\hline ENSG00000117616 & 2.98 & C1orf63 & Chromosome 1 open reading frame 63 \\
\hline ENSG00000204691 & 3.04 & Q9H355_HUMAN & $\begin{array}{l}\text { metastasis associated lung adenocarcinoma transcript } 1 \text { (non- } \\
\text { coding RNA) }\end{array}$ \\
\hline ENSG00000188170 & 4.01 & HBB & Hemoglobin, beta \\
\hline
\end{tabular}



